\title{
The Differential Cross Section Distribution of Drell-Yan Dielectron Pairs in the Z Boson Mass Region
}

\author{
by \\ Jiyeon Han
Submitted in Partial Fulfillment of the
Requirements for the Degree of
Doctor of Philosophy

Supervised by

Professor Arie Bodek and Dr. Willis Sakumoto

Physics and Astronomy

The College

Arts and Sciences

University of Rochester

Rochester, New York

2008 


\section{Curriculum Vitae}

The author was born in Seoul, Korea on September 19, 1976. She attended Yonsei University from 1996 to 2000 and graduated with a Bachelor of Science degree in physics in 2000. She started her graduate studies in Yonsei University afterward and achieved the Master of Science degree in Yonsei University in 2002. The main research was High Energy Particle Physics.

She came to the University of Rochester in the Fall of 2003 and began graduate studies in Physics. She pursued her research in High Energy Particle Physics under the direction of Professor Arie Bodek and Dr. Willis Sakumoto. She received the Master of Science degree from the University of Rochester in 2005. 


\section{Acknowledgements}

I was very lucky to conduct my Ph.D program as a member of University of Rochester in CDF. There were many experts around me and I could get their helps and advice whenever I wanted. I was able to extend and develop my knowledge about high energy physics thanks to help from these people.

First of all, I am extremely thankful to my supervisors, Arie Bodek and Willis Sakumoto. They have always been so supportive during the entire period of my Ph.D. Arie and Willis have provided me with valuable discussions and advice. Without their helps, I could not finish my work. Especially, Willis has spent enormous time for teaching me and discussing data analysis with me. It was a great experience to learn many new ideas from him and solve many problems with him. It has inspired and motivated me very much.

In addition, I would like to thank the CDF electroweak physics group and W/Z cross section group conveners for their generous helps. Special thanks to Pasha Murat,

David Simon Waters, Chris Hays, Eric B. James, Eva Halkiadakis, Mark Lancaster, Larry Nodulman, and Aidan Robson.

I thank Kaori Maeshima, Ted Liu, and Veronica Sorin. Kaori helped me undertake CDF service work as a member of online data taking, as which I had a great opportunity to learn from Ted and Veronica about the CDF trigger system.

Also, I would like to thank other Rochester members and my classmates, Howard Budd, Yonsei Chung, Kevin McFarland, Jedong Lee, Boyoung Han, Guembong You, Jennifer Gimmell and Su-Jung Park.

Howard Budd and Yonsei Chung have helped me solve my curiosity and questions. 
Jedong Lee and Boyong Han have gladly shared their knowledge with me. I had a great time during my Ph.D course thanks to my classmates. Special thanks to SuJung. I could not enjoy the graduate student life without her.

And I sincerely appreciate the Rochester physics department people: Mrs. Barbara Warren, Mrs, Connie Jones, Mrs. Sue Brightman, and Mrs. Judy Mack. They have taken care of me as if I were their family member since I started in graduate school.

Now I have to thank my family, especially my parents. They always encouraged my study and their love made me overcome any difficulties so far. I could not focus on my study without their devotion.

And my husband, Hyunwoo Kim, he has always stood by me and supported my study. I received much encouragement from him to finish this study.

There are still many more people I have to thank. I am grateful to all of those friends and colleagues who helped me finish my study. 


\section{Abstract}

We report on a measurement of the rapidity distribution, $d \sigma / d y$, for $Z /$ Drell-Yan $\rightarrow e e$ events produced in $p \bar{p}$ collisions at $\sqrt{s}=1.96 \mathrm{TeV}$. The data sample consists of 2.13 $\mathrm{fb}^{-1}$ corresponding to about about $160,000 \mathrm{Z}$ /Drell-Yan $\rightarrow$ ee candidates in the $Z$ boson mass region collected by the Collider Detector at Fermilab. The $d \sigma / d y$ distribution, which is measured over the full kinematic range for $e^{+} e^{-}$pairs in the invariant mass range $66<M_{e e}<116 \mathrm{GeV} / c^{2}$, is compared with theory predictions. There is good agreement between the data and predictions of Quantum Chromodynamics in Next to Leading Order with the CTEQ6.1M Parton Distribution Functions. 


\section{Contents}

Curriculum Vitae ii

Acknowledgements $\quad$ iii

Abstract $\quad$ V

List of Tables $\quad$ ix

List of Figures $\quad$ xi

1 Introduction 1

1.1 Standard Model . . . . . . . . . . . . . . . . . . . . . . . 1

1.2 The Drell-Yan Process $: p \bar{p} \rightarrow \gamma^{*} / Z \rightarrow \ell^{+} \ell^{-} \ldots \ldots . \ldots . \ldots$

1.2.1 Parton Distribution Function (PDFs) . . . . . . . . . 4

1.2.2 $d \sigma / d y$ Distribution of $\gamma^{*} / Z$ Boson . . . . . . . . . . . 5

1.2.3 Overview of the Analysis . . . . . . . . . . . . . . . . 10

2 Experimental Apparatus $\quad 12$

2.1 The Fermilab Accelerator Complex . . . . . . . . . . . . . . . . . 12

2.2 The Collider Detector at Fermilab . . . . . . . . . . . . . . . . 14

2.2.1 The Silicon Tracker . . . . . . . . . . . . . . . . . . 16

2.2.2 The Central Outer Tracker . . . . . . . . . . . . . . 19

2.2.3 The Calorimeters . . . . . . . . . . . . . . . 21

2.3 The Trigger System . . . . . . . . . . . . . . . . . . 26

2.3.1 Level-1.................... 26

2.3 .2 Level-2 . . . . . . . . . . . . . . . . . . . . . . 28 
$2.3 .3 \quad$ Level-3 . . . . . . . . . . . . . . . . . . . .

3 Data $\quad 32$

3.1 Data Sample. . . . . . . . . . . . . . . . . . . . . . . . 32

3.2 Monte Carlo Sample . . . . . . . . . . . . . . . . . . . . . . . . . . . 32

3.3 Electron Candidates . . . . . . . . . . . . . . . . . . 33

3.3.1 Electron Identification . . . . . . . . . . . . . . . . 33

3.4 Z Event Selection . . . . . . . . . . . . . . . . . 39

3.5 Energy Correction . . . . . . . . . . . . . . 43

3.5.1 PPR Energy . . . . . . . . . . . . . . 43

3.5.2 Plug Electron Energy Calibration in Data . . . . . . . . . . . 43

3.5.3 Energy Scale in MC . . . . . . . . . . . . . 45

3.6 The ZVertex Correction in $\mathrm{MC} \ldots \ldots$. . . . . . . . . . 60

3.7 Trigger Requirements and Efficiencies . . . . . . . . . . . . . 64

3.7.1 Trigger Requirements . . . . . . . . . . . . . 64

3.7 .2 Trigger Efficiencies . . . . . . . . . . . . . 64

4 Background $\quad 72$

4.1 Isolation Extrapolation Method . . . . . . . . . . . . 73

4.1.1 QCD Backgrounds ................. 73

4.1.2 Signal and Background Template . . . . . . . . . . . . 74

4.1 .3 IsolationE Fit . . . . . . . . . . . . . . . . . . 77

4.2 The Z/Drell-Yan dielectron Mass Fit Method _ . . . . . . . . . . . 90

4.2.1 Mass distribution of QCD $\gamma+$ jet events . . . . . . . . . 92

4.2.2 Fitting the Z/Drell-Yan dielectron Mass Distribution . . . . . 96

4.3 Systematic Uncertainty . . . . . . . . . . . . . . . . . . . 99

4.4 Total Background Rate . . . . . . . . . . . . . . . . 110

4.4.1 Electroweak Background . . . . . . . . . . . . . . . . 110

5 Acceptance and Efficiency $\quad 115$

5.1 Geometric and Kinematic Acceptance . . . . . . . . . . . . . . . 115 
5.2 Phoenix Tracking Efficiency _. . . . . . . . . . . . . . . . 117

5.2.1 The ZVertex Reconstruction Efficiency . . . . . . . . . . . 121

$5.2 .2 \quad$ ZVertex Selection . . . . . . . . . . . . . . . . . 126

5.2.3 Phoenix Tracking Efficiency Dependence on Instantaneous Luminosity . . . . . . . . . . . . . . . . . . . . 129

5.2.4 Events with a ZVertex but No Track . . . . . . . . . . 129

5.2 .5 Events with Tracks . . . . . . . . . . . . . . . . 131

5.2.6 Phoenix Tracking Efficiency versus Boson Rapidity . . . . . . 133

5.3 Electron Identification Efficiency . . . . . . . . . . . . . . . 136

5.3.1 Electron Identification Efficiency versus Time . . . . . . . . 137

5.3.2 Central Electron Identification Efficiency . . . . . . . . . . . 139

5.3.3 Plug Electron Identification Efficiency in Z $(\mathrm{CP}) \quad \ldots \ldots \ldots$

5.3.4 Plug Electron Identification Efficiency in Z(PP) Events . . . . 142

5.3.5 Identification Efficiency in Boson Rapidity . . . . . . . . . . 143

6 Systematic Uncertainties 146

7 Results 152

7.1 The $d \sigma / d y$ Distribution for the $\gamma^{*} / Z \rightarrow e e$ Process $\ldots \ldots \ldots \ldots$

7.1.1 Acceptance Correction . . . . . . . . . . . . . . . 153

$7.1 .2 d \sigma / d y_{Z}$ Distribution $\ldots \ldots \ldots \ldots \ldots \ldots \ldots$

7.2 Comparison of Data to Theory _ . . . . . . . . . . . 158

$\begin{array}{lll}8 & \text { Conclusion } & 163\end{array}$

$\begin{array}{ll}\text { A Glossary } & 166\end{array}$

$\begin{array}{ll}\text { Bibliography } & 168\end{array}$ 


\section{List of Tables}

1.1 Organization of Fermions . . . . . . . . . . . . . . . . . 2

2.1 COT Mechanical Summary . . . . . . . . . . . . . . 20

3.1 Central Electron Selection . . . . . . . . . . . . . . 40

3.2 Plug Electron Selection . . . . . . . . . . . . . . . . 41

3.3 Mean and Width of Z Mass . . . . . . . . . . . . . . . 56

3.4 Mean and Width of Z Mass . . . . . . . . . . . . . . . . . . . 61

3.5 Trigger Path Requirements . . . . . . . . . . . . . . . 65

3.6 Number of Events in Each topology After Selection . . . . . . . . . . 71

4.1 The Selection of Signal and Background Templates . . . . . . . . 78

4.2 IsolationE Fit Range . . . . . . . . . . . . . . . . . . . . . . . 82

4.3 Background Fraction Summary in Isolation Extrapolation Method . . 89

4.4 Background Fraction in Signal Sample . . . . . . . . . . . . . . 90

4.5 Background Rate in Z Mass Fit . . . . . . . . . . . . . . . . . . 100

4.6 Slope of $\gamma+$ jet Mass Distribution with Different Assumption on E/P 106

4.7 Background Fraction in 2-Track Events . . . . . . . . . . . . . . 107

4.8 Background Rate for Systematic Uncertainty . . . . . . . . . . . . . . 108

4.9 Background from Electroweak Processes . . . . . . . . . . . . . 113

5.1 Kinematic Selection for Acceptance . . . . . . . . . . . . . . . 117

5.2 Reconstructed ZVertex Inefficiency . . . . . . . . . . . . . . . . . 124

5.3 Fraction of Events in No-Track Events . . . . . . . . . . . . . . . . . 132 
5.4 Fraction and Tracking Efficiency in Expected Silicon Layers . . . . . 134

5.5 Phoenix Tracking Efficiency of Electron . . . . . . . . . . . . . . . 135

5.6 Weighting Factor of Tracking Efficiency . . . . . . . . . . . . 136

7.1 Table of $d \sigma / d y$ Measurement . . . . . . . . . . . . . . . . . . . . . . . 162

8.1 Number of Signal and Background Events in Each Topology . . . . . 165 


\section{List of Figures}

1.1 Drell-Yan Process . . . . . . . . . . . . . . . . . . . . 6

1.2 Parton Distribution Functions . . . . . . . . . . . . . 7

1.3 The Relation between the Parton Momentum Fractions and Rapidity 9

2.1 The accelerator chain of Tevatron at Fermilab . . . . . . . . 14

2.2 A side view of the CDF Run II Detector . . . . . . . . . . 16

2.3 Various Particles Decay Chart . . . . . . . . . . . . . . 17

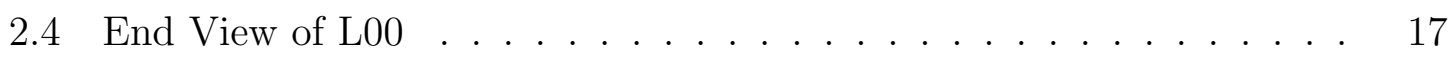

2.5 End View of SVX Bulkhead . . . . . . . . . . . . . . 18

2.6 Three COT Cells . . . . . . . . . . . . . . . . . . . . . . . . 21

2.7 A view of a portion of one COT endplate . . . . . . . . . 22

2.8 A Side View of Tracking System . . . . . . . . . . . . . . . 23

2.9 One Wedge of the Central Calorimeter . . . . . . . . . . . . 24

2.10 A Side View of Plug Calorimeter . . . . . . . . . . . 26

2.11 Schematic Drawing of a Tile-Fiber Calorimeter . . . . . . . . 27

2.12 Data Flow of Trigger and DAQ . . . . . . . . . . . . . 30

2.13 L1 and L2 Trigger Subsystem _. . . . . . . . . . . . . 31

$3.1 Z / \gamma^{*}$ Boson Mass and Rapidity Distribution . . . . . . . . . 42

3.2 PPR Energy Distribution . . . . . . . . . . . . . . . 44

3.3 Plug Electron Energy Calibration in Data . . . . . . . . . . . 44

3.4 Central Electron Energy Scale in $\mathrm{Z}(\mathrm{CC}) \ldots \ldots \ldots$

$3.5 \quad E_{T}$ Distribution in $\mathrm{Z}(\mathrm{CC}) \ldots \ldots \ldots \ldots \ldots$ 
$3.6 \mathrm{Z}(\mathrm{CC})$ Mass Distribution . . . . . . . . . . . . . . . . . . 48

3.7 Central Electron Energy Scale in $\mathrm{Z}(\mathrm{CP}) \ldots \ldots \ldots \ldots$

$3.8 E_{T}$ Distribution of Central Electrons in $\mathrm{Z}(\mathrm{CP}) \ldots \ldots \ldots \ldots$

$3.9 \mathrm{Z}(\mathrm{CP})$ Mass Distribution with Energy Scale in Central Electron . . . 52

3.10 Plug Electron Energy Scale in $\mathrm{Z}(\mathrm{CP}) \ldots \ldots \ldots \ldots$

$3.11 E_{T}$ Distribution of Plug Electron in $\mathrm{Z}(\mathrm{CP}) \ldots \ldots \ldots \ldots$

$3.12 \mathrm{Z}(\mathrm{CP})$ Mass Distribution with Energy Scale in Plug Electron . . . . . 55

3.13 Plug Electron Energy Scale in $\mathrm{Z}(\mathrm{PP}) \ldots \ldots \ldots \ldots . \ldots . \ldots 57$

$3.14 E_{T}$ Distribution of Plug Electron in $\mathrm{Z}(\mathrm{PP}) \ldots \ldots \ldots \ldots$

3.15 Z(PP) Mass Distribution . . . . . . . . . . . . . . . . 59

3.16 Ratio of Data to MC in ZVertex . . . . . . . . . . . 63

$3.17 Z_{-}$NOTRACK Trigger Efficiency . . . . . . . . . . . 68

3.18 ELECTRON_CENTRAL_18 Trigger Efficiency . . . . . . . . . 69

3.19 Trigger Efficiency in Boson Rapidity . . . . . . . . . . . . 70

4.1 The Isolation Extrapolation Method _ . . . . . . . . . . . . 74

4.2 MET Distribution of Background Template . . . . . . . . . . 77

4.3 MET Distribution of Background Template for Central Electron . . . 79

4.4 MET Distribution of Background Template for Plug Electron _ . . . 80

4.5 IsolationE Difference between Track and No-Track Leg in $\mathrm{Z}(\mathrm{CP}) \ldots 85$

4.6 IsolationE Fit in $\mathrm{Z}(\mathrm{CC}) \ldots \ldots \ldots \ldots \ldots$

4.7 IsolationE Fit for the Central Electron in $\mathrm{Z}(\mathrm{CP}) \ldots \ldots \ldots \ldots$

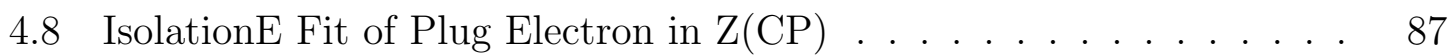

4.9 IsolationE Fit of Track Leg in $\mathrm{Z}(\mathrm{PP}) \ldots \ldots \ldots \ldots$

4.10 IsolationE Fit of Non-Track Leg in $\mathrm{Z}(\mathrm{PP}) \ldots \ldots \ldots$

$4.11 \eta$ Distribution of the Plug Electron in Data, and for the Photon in $\mathrm{QCD} \gamma+$ jet $\mathrm{MC} \ldots \ldots \ldots \ldots \ldots \ldots \ldots \ldots \ldots$

4.12 Phase-Space of Fragmentation Energy Fraction . . . . . . . . 95

4.13 Mass Distribution of QCD $\gamma+$ jet Events in Simulation . . . . . . 97

4.14 Z Mass Fit in $\mathrm{Z}(\mathrm{CC}) \ldots \ldots \ldots \ldots$ 
$4.15 \mathrm{Z}$ Mass Fit in $\mathrm{Z}(\mathrm{CP}) \ldots \ldots \ldots \ldots \ldots$

$4.16 \mathrm{Z}$ Mass Fit in $\mathrm{Z}(\mathrm{PP}) \ldots \ldots \ldots \ldots$

4.17 Fragmentation Energy Fraction Systematics _ . . . . . . . . . 105

4.18 Rapidity Distribution of QCD Background in Each Topology . . . . . 109

4.19 QCD Background Distribution in Rapidity . . . . . . . . . . . 111

4.20 Rapidity Distribution in Data . . . . . . . . . . . . . . . 113

4.21 Rapidity Distribution of Data and Backgrounds . . . . . . . . . 114

$5.1 d \sigma / d y$ of Theory Predictions . . . . . . . . . . . . . . 118

5.2 Acceptance Ratio with Correction from Predictions . . . . . . . . . 119

5.3 Geometric and Kinematic Acceptance . . . . . . . . . . . . . . 120

5.4 The silicon detector geometry including the ISL. . . . . . . . . . . 121

5.5 ZVertex from Mass Constrained Method . . . . . . . . . . . . . . . 124

5.6 Z Mass Distribution of Events without Reconstructed ZVertex . . . . 125

5.7 Z Mass Distribution of Events with Reconstructed ZVertex . . . . . 126

5.8 ZVertex Selection . . . . . . . . . . . . . . . . . . 128

5.9 Z Mass of No Track Events . . . . . . . . . . . . . . . . . . . . . 131

5.10 Fraction of Events in Fiducial Range . . . . . . . . . . . . . 133

5.11 Tracking Efficiency in $\mathrm{Z}(\mathrm{PP}) \ldots \ldots \ldots \ldots \ldots$

5.12 Event Rate in Time . . . . . . . . . . . . . . . . . . . . . . 138

5.13 Central Electron ID Efficiency . . . . . . . . . . . . . . . 140

5.14 Plug Electron ID Efficiency in Z(CP) . . . . . . . . . . . 142

5.15 Plug Electron ID Efficiency for Z(PP) . . . . . . . . . . . . 144

5.16 ID Efficiency in Boson Rapidity . . . . . . . . . . . . . . . 145

6.1 Detector Material Modeling . . . . . . . . . . . . . . . . . 147

6.2 Fractional Systematic Uncertainty in Boson Rapidity . . . . . . . . 151

7.1 Ratio of $d N / d y_{Z}$ in data to prediction . . . . . . . . . . . 154

7.2 Acceptance Ratio of Final Corrections to Correction before Iteration Process . . . . . . . . . . . . . . . . . . . . . 155 
7.3 Rapidity Distribution in the data . . . . . . . . . . 156

$7.4 d \sigma / d y$ Distribution in $y_{Z} \geq 0$ and $y_{Z}<0 \ldots \ldots \ldots \ldots \ldots$

$7.5 d \sigma / d y$ Distribution . . . . . . . . . . . . . . . . 157

$7.6 d \sigma / d y$ Distribution in $\mathrm{Z}(\mathrm{CC}), \mathrm{Z}(\mathrm{CP})$, and Z(PP) Topology $\ldots \ldots . .158$

$7.7 d \sigma / d y$ Ratio of the data to Theory Prediction $\ldots \ldots \ldots \ldots$

$7.8 d \sigma / d y$ Ratio of the data to Theory Prediction in $y \geq 0$ and $y<0 \ldots 161$ 


\section{Chapter 1}

\section{Introduction}

\section{$1.1 \quad$ Standard Model}

The Standard Model (SM) is a theoretical framework that describes how matter is composed of fundamental constituents, and the various types of interactions between these constituents. In the SM, the fundamental constituents of matter are fermions, of which there are twelve different types. These fermion constituents are categorized in three families (or generations) of quarks and leptons of increasing mass. All the SM fermion constituents are 1/2 spin. Fermions follow the Pauli exclusion principle in accordance with the spin-statistics theorem. Table 1.1 shows the three families of fermion constituents of matter. Particles in higher generations have heavier mass and are unstable. Therefore, particles in higher generations decay into particles in lower generations conserving quantum numbers (Charge, Lepton number, Baryon Number, and Spin). The different quark and leptons in Table 1.1 are characterized as having different flavors.

Particles of matter interact with each other via four fundamental forces. These are the electromagnetic, weak, strong, and gravitational interactions. The four interactions are mediated by boson field particles. The boson field particles in the SM 


\begin{tabular}{|c|c|c|c|}
\hline & Generation 1 & Generation 2 & Generation 3 \\
\hline \hline \multirow{2}{*}{ Quarks } & $\mathrm{u}(\mathrm{up})$ & $\mathrm{c}($ charm) & $\mathrm{t}($ top) \\
& $\mathrm{d}($ down) & $\mathrm{s}$ (strange) & $\mathrm{b}$ (bottom) \\
\hline \hline \multirow{2}{*}{ Leptons } & $\mathrm{e}$ & $\mu$ & $\tau$ \\
& $\nu_{e}$ & $\nu_{\mu}$ & $\nu_{\tau}$ \\
\hline
\end{tabular}

Table 1.1: The three generations of fermion constituents of matter in the Standard Model.

are of integer spin. The field particles of the electromagnetic, weak and strong interactions have spin $=1$. The graviton, which is the field particle of the gravitational force is believed to have spin $=2$. The massless photon mediates the electromagnetic interaction between electrically charged particles. The electromagnetic interaction is well described by the theory of quantum electrodynamics (QED). The massive $W^{ \pm}$ and $Z$ gauge bosons are the field particles which mediate the weak interaction (W bosons mediate the charged-current interaction which changes the flavor of the particle). Both gauge bosons are massive, and the $Z$ is heavier than the $W^{ \pm}$boson. The W bosons carries a electric charge of +1 and -1 , and therefore couple to the electromagnetic interaction as well. In the SM, the weak and electromagnetic interaction are unified within the Glashow-Weinberg-Salam electroweak theory.

Field particles which are called gluons mediate the strong force which describes the interaction between particles having a quantum number called color. The strong force is described by the theory of quantum chromodynamics (QCD). The color charge of the quarks are described as R(red), G(green), and B(blue). Gluons, which also carry color charges, interact with the quarks and change their color charge. In addition, since gluons themselves carry a color charge, gluons also interact with other gluons. The theory of QCD is similar to QED in terms that both have a massless gauge 
boson, namely the gluon in QCD and the $\gamma$ in QED. However, QED theory has only single "charge", which is the electromagnetic charge and is described in group theory by the $\mathrm{U}(1)$ group. In QCD, three color charges participate in the interaction which are described in group theory by $\mathrm{SU}(3)$ color group. The gluon also carries a color charge of the $\mathrm{SU}(3) \times \mathrm{SU}(3)$ variety, and this results in 8 different types gluon.

The SM also contains a complex, scalar doublet field, which is commonly referred to as the Higgs field, originally proposed in 1964 in three theoretical papers in Physical Review Letters by Peter W. Higgs, and by G.S. Guralnik, C.R. Hagen, and T.W.B. Kibble, and by F. Englert and R. Brout. The Higgs field has a non-zero vacuum expectation value which spontaneously breaks the electroweak gauge symmetry. This mechanism responsible for giving mass to the $\mathrm{W}$ and $\mathrm{Z}$ bosons, also predicts the existence of yet unseen Higgs bosons . The SM does not predict the exact mass of the Higgs bosons, but it is expected to be massive.

Even though the SM is a very successful framework in explaining matter and the three types of interactions, it does not include a description of the gravitational interaction. Many theoretical and experimental approaches have been tried to extend the SM to include gravity and/or additional new interactions. These extensions are referred to as physics "Beyond the Standard Model".

\subsection{The Drell-Yan Process $: p \bar{p} \rightarrow \gamma^{*} / Z \rightarrow \ell^{+} \ell^{-}$}

In high energy nucleon-nucleon scattering, a quark from one hadron and an antiquark from another hadron can annihilate to create a pair of oppositely charged leptons through the exchange of a virtual photon $\left(\gamma^{*}\right)$, or the exchange of a $\mathrm{Z}$ boson. This is usually referred to as the Drell-Yan process[2]. The Drell-Yan process provides 
valuable information on parton (quark and gluon) distribution functions (PDFs) in the nucleon and provides a stringent test of perturbative QCD. The PDFs describe the momentum distribution of quarks and gluons in hadrons. The Drell-Yan process is the s-channel analog of deep inelastic lepton-nucleon scattering(DIS). The DrellYan process has a clear theoretical interpretation as quark-antiquark annihilation into vector bosons. The event rate of the Drell Yan process is large, and the two leptons in the final state provide a clear experimental signature. Therefore, the Drell-Yan process can be considered as one of the golden-processes for extracting information on PDFs.

\subsubsection{Parton Distribution Function (PDFs)}

The parton momentum fraction $(x)$ is the momentum fraction which a parton (quark, antiquark, or gluon) carries inside a fast moving hadron. The parton distribution functions provide the probability density distribution of quarks and gluons as a function of both the momentum fraction, $x$, and a perturbative QCD scale, $Q^{2}$.

The PDFs are used to calculate the cross section of all physical processes in hadron-hadron collisions. In the parton model, the cross section is the incoherent sum of all partonic cross sections :

$$
\sigma=\sum_{i j} \int d x_{i} \int d x_{j} f_{j}\left(x_{j}, Q^{2}\right) f_{i}\left(x_{i}, Q^{2}\right) \hat{\sigma}\left(x_{i}, x_{j}, Q^{2}\right)
$$

where $f_{i}\left(x_{i}, Q^{2}\right)$ is the probability density function of the parton $i$ in the hadron. and $\hat{\sigma}\left(x_{i}, x_{j} ; Q^{2}\right)$ is the partonic cross section for a given scattering process, which is computed within perturbative QCD. The sum runs over all possible partons which participate in the process. The $Q^{2}$ scale arises from factorizing the QCD calculation into a hard scattering part that can be calculated via perturbative QCD and a soft, 
incalculable part, the parton distribution functions. QCD theory cannot predict the values of PDFs as a function of $x$. However, the scaling behavior in $Q^{2}$ is calculable. Therefore, the nucleon PDFs need to be measured. The PDFs are basic ingredients in understanding nucleon structure. It is not possible to predict the PDFs within perturbative QCD because of the inherent non-perturbative effects in a QCD bound state. At present, even the most recent non-perturbative lattice QCD calculations are unable to calculate the nucleon structure functions. Therefore, parton distribution functions are obtained using experimental data. Specially, PDFs have been extracted from lepton-nucleon deep inelastic scattering (DIS) data. In DIS experiments, the lepton beam (electrons, muons or neutrinos) is scattered off a nucleon target. The distribution of the final state momentum of the scattered leptons is used to investigate the unknown structure of the target nucleons. Each DIS experiment measures the structure function of the target particle in a limited range of $\mathrm{x}$ and $Q^{2}$ space. The measurements (which vary in precision) for many different processes are combined in "global QCD fits" to extract PDFs for all parton species in the nucleon. The CTEQ and MRST collaborations have provided the most commonly used PDFs from their "global QCD fits". Figure 1.2 shows the PDFs generated by the CTEQ6.1M parton distribution set.

\subsection{2 $d \sigma / d y$ Distribution of $\gamma^{*} / Z$ Boson}

The rapidity $(y)$ is a useful kinematic property of a final state particle and is defined as

$$
y \equiv \frac{1}{2} \ln \left(\frac{E+P_{z}}{E-P_{z}}\right)
$$

where $E$ is the energy of the final state particle, and $P_{z}$ is its momentum along $z$ axis. 


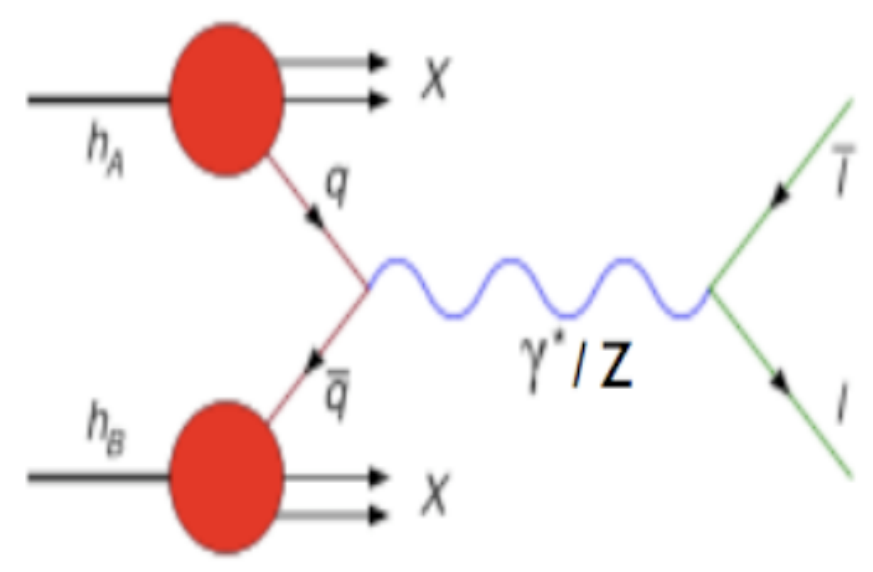

Figure 1.1: The Drell-Yan process .

In $p \bar{p}$ collision, the final state rapidity of a particle is related to the initial parton momentum fractions of the quark and antiquark which participate in the interaction as follows:

$$
x_{1,2}=\frac{M_{Z}}{\sqrt{s}} e^{ \pm y}
$$

where $x_{1,2}$ are the momentum fraction of the quark and antiquark, $M_{Z}$ is the $\mathrm{Z}$ boson mass, and $\sqrt{s}$ is the total center of mass energy for the collision. Figure 1.3 shows this relation for $p \bar{p}$ collision at $\sqrt{s}=1.96 \mathrm{TeV}$. The parton momentum fractions, $x_{1,2}$ determine the rapidity of final state $\mathrm{Z}$ boson. High rapidity corresponds to high $x_{1}$ and low $x_{2}$. Therefore, a measurement of the rapidity distribution in the high y region probes the PDFs in the very high and very low $\mathrm{x}$ regions, which are not known with high precision. In addition, in QCD at higher orders (NLO or NNLO), interactions involving gluons in the initial state also contribute to the rapidity distribution. At low $\mathrm{x}$, since the density distribution of gluons and sea quarks is large, the Drell -Yan process is sensitive to higher order QCD corrections. The HERA collider is an ep 


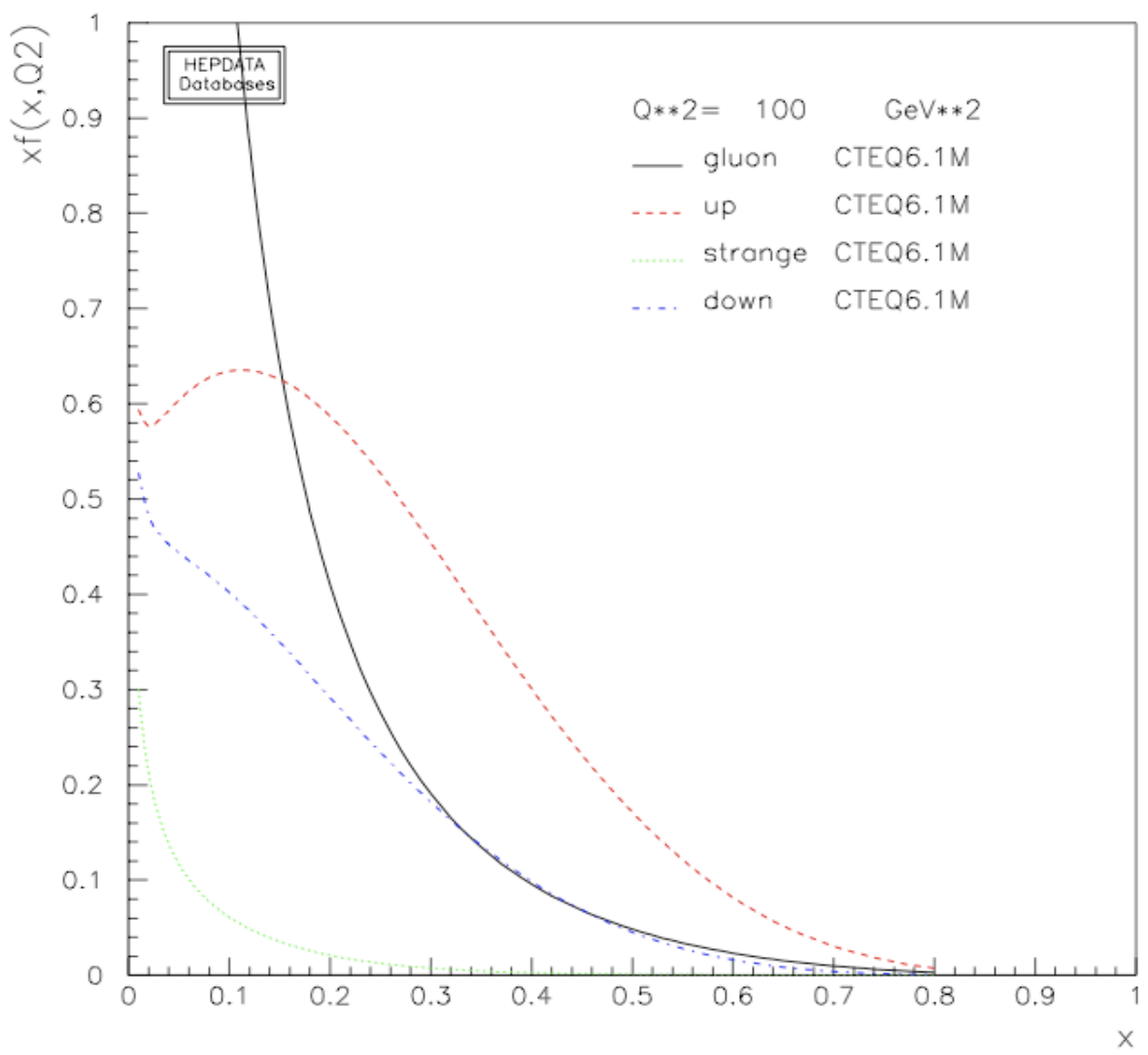

Figure 1.2: The parton distribution functions in CTEQ6,1M. 
collider located at the DESY laboratory in Hamburg. At HERA, collisions of 28 $\mathrm{GeV} e^{ \pm}$with $820 \mathrm{GeV}$ protons are used to measure the proton structure functions at small x at higher values of $Q^{2}$. Measurements of the structure functions extending in $Q^{2}$ up to $10^{4} \mathrm{GeV}^{2}$ have been done by the two principal HERA detectors, H1 and ZEUS. The data collected at the HERA ep collider has been used to extract the $\mathrm{x}$ distributions of valence quarks, sea quarks and gluons as a function of $x$ and $Q^{2}$.

In $p \bar{p} \rightarrow \gamma^{*} / Z\left(\rightarrow \ell^{+} \ell^{-}\right)$process, the differential cross section as a function of rapidity can be written as

$\frac{d \sigma}{d y d M^{2}}=\sum_{i, j} \hat{\sigma}\left(i j \rightarrow \gamma^{*} / Z\right) \int_{x_{1}}^{1} d x_{i} \int_{x_{2}}^{1} d x_{j} f_{j / p}\left(x_{j}, Q^{2}\right) f_{i / p}\left(x_{i}, Q^{2}\right) \Delta_{i j}\left(x_{i}, x_{j}, x_{1}, x_{2}, Q^{2}\right)$

where index $i$ and $j$ refer to the partons $(q, \bar{q}$, or $g)$ inside the proton or antiproton, which participate in the process. Here, $\hat{\sigma}\left(i j \rightarrow \gamma^{*} / Z\right)$ is the partonic cross section of the Drell-Yan process, $f_{i / p}\left(x_{i}, Q^{2}\right) d x_{i}$ is the probability of finding a parton $i$ inside the proton with a fractional momentum between $x_{i}$ and $x_{i}+d x_{i}$, and $\Delta_{i j}$ is the perturbative QCD coefficient function for the Drell-Yan process. The partonic cross section of Drell-Yan process $\left(\hat{\sigma}\left(i j \rightarrow \gamma^{*} / Z\right)\right)$ is calculable in theory and it can be written as

$$
\hat{\sigma}\left(i j \rightarrow \gamma^{*} / Z\right)=\frac{4 \pi \alpha^{2}}{9 Q^{2}} \sum_{i, j}\left(e_{i}^{2}+e_{j}^{2}\right)
$$

where $e_{i}\left(e_{j}\right)$ is the quark charge of parton $i(j)$ and $\alpha$ is fine structure constant $\left(\alpha=e^{2} /(4 \pi)=1 / 137\right)$. In massive lepton pair production in hadron-hadron collisions, the process is

$$
p_{1}+p_{2} \rightarrow \gamma^{*} / Z\left(\rightarrow \ell_{1}+\ell_{2}\right)+X
$$

where " $\mathrm{X}$ " denotes any inclusive hadronic final state which is allowed by conservation of quantum numbers. In higher order QCD, X can be any multi partonic state, and 


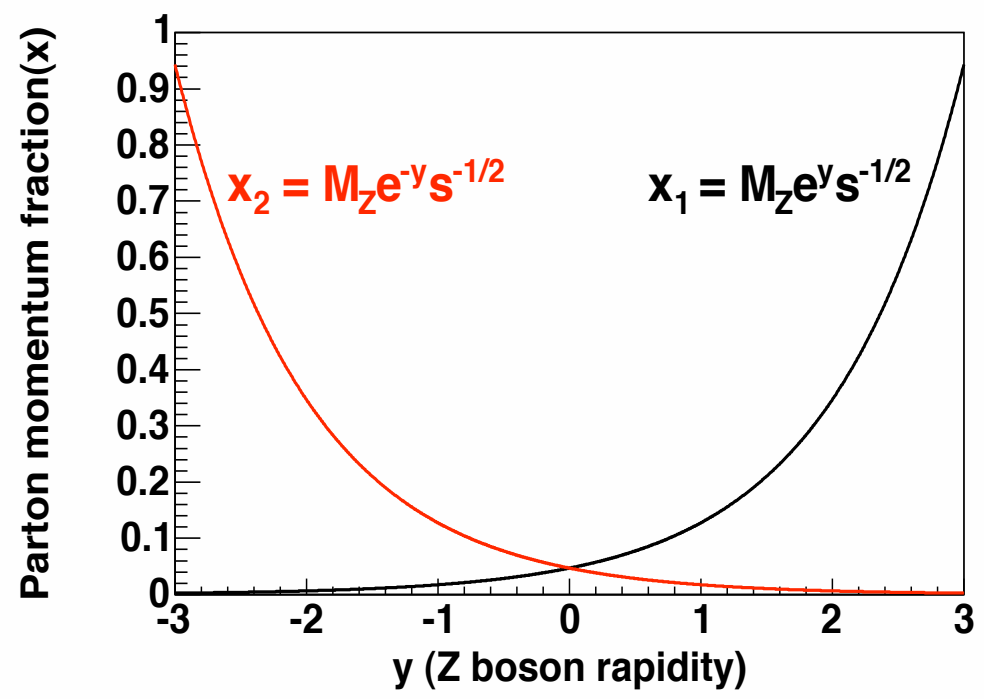

Figure 1.3: The relation of the parton momentum fraction of the quark and $\operatorname{antiquark}\left(x_{1,2}\right)$ and the $Z$ boson rapidity $(\mathrm{y})$.

the coefficient function $\Delta_{i j}$ is the convolution kernel for the full pQCD description of the hard parton-parton collision.

The term $\Delta_{i j}$ can be expanded as a power series in the running coupling constant $\alpha_{s}\left(\mu^{2}\right)$ as follows

$$
\Delta_{i j}=\sum_{n=0}^{\infty}\left(\frac{\alpha_{s}\left(\mu^{2}\right)}{4 \pi}\right)^{n} \Delta\left(\mu^{2}, Q^{2}\right)
$$

In lowest order QCD, the coefficient function is determined by the subprocess

$$
q+\bar{q} \rightarrow \gamma^{*} / Z
$$

In this case, the coefficient function is given by

$$
\Delta_{q \bar{q}}^{(0)}=\delta\left(x_{i}-x_{1}\right) \delta\left(x_{j}-x_{2}\right)
$$


In $\mathrm{QCD} \mathcal{O}\left(\alpha_{s}\right)$ correction (NLO), the gluon bremsstrahlung process, and the process with gluon in the initial state contribute to the coefficient function.

$$
\begin{aligned}
& q+\bar{q} \rightarrow \gamma^{*} / Z+g \\
& g+q(\bar{q}) \rightarrow \gamma^{*} / Z+q(\bar{q})
\end{aligned}
$$

Both contributions $\Delta_{q \bar{q}}^{(1)}$ and $\Delta_{g q}^{(1)}$ have been calculated in both the QCD DIS-scheme[3], [4], [5], and the QCD $\bar{M} S$-scheme[6].

Though various partial calculations on the NNLO differential cross section have been done $\left(d \sigma / d M^{2}\right)$, no complete calculation of the NNLO QCD corrections has been performed[8]. Recently, a new technique has been developed to calculate $d^{2} \sigma / d y d M^{2}$ at NNLO. In this new method, the optical theorem is extended in such a way that the calculation of differential distributions becomes possible using techniques developed for multi-loop calculations. [9]

\subsubsection{Overview of the Analysis}

In this thesis, We report on a measurement of the rapidity distribution, $d \sigma / d y$, for $Z$ bosons produced in $p \bar{p}$ collisions at $\sqrt{s}=1.96 \mathrm{TeV}$. The data sample consists of 2.1 $\mathrm{fb}^{-1}$ collected by the Collider Detector at Fermilab (CDF). The $d \sigma / d y$ distribution, which is measured over the full kinematic range for $e^{+} e^{-}$pairs in the $Z$ boson mass range $66<M_{e e}<116 \mathrm{GeV} / c^{2}$, is compared with theory predictions. Throughout this thesis, we use the term electron to refer to both electrons or positrons.

The CDF detector is instrumented with electromagnetic calorimeters in both the central and forward regions. Z events with two electrons in forward region correspond to the high rapidity region. Therefore, we can probe a larger high $x$ region by including electron pairs in the forward region. Electrons in the central region are associated with a good track because the central region is fully covered by the central tracker. 
However, the forward region is not covered completely by the tracker. Therefore, a special tracking algorithm, which includes the silicon vertex detector, is used in the forward region. By requiring a track for at least one of the two electrons in the forward region, we can reduce the background contamination, and also determine the interaction vertex along the $z$ axis. The measurement in the high rapidity region provides extended kinematic coverage for $Z$ boson events. However, this is also the region where the background contamination is expected to be larger. Therefore, we have developed two independent methods that provides a better understanding of the background.

The main goal of this analysis is to measure the differential cross section as a function of rapidity and to compare it with $\mathrm{QCD}$ theory predictions. We compare the normalized $d \sigma / d y$ distribution (which is divided by the total cross section), to theoretical predictions. The theory predictions are calculated in both NLO and NNLO QCD using both MRST and CTEQ PDFs. 


\section{Chapter 2}

\section{Experimental Apparatus}

The Fermi National Accelerator Laboratory (Fermilab) is located 30 miles west of Chicago, Illinois. Fermilab, originally named the National Accelerator Laboratory, was commissioned by the U.S. Atomic Energy Commission on November 21, 1967. On May 11, 1974, the laboratory was renamed in honor of 1938 Nobel Prize winner Enrico Fermi. Fermilab has many scientific programs, including Tevatron collider experiments (CDF and D0), neutrino experiments (MiniBooNE, MINOS, MINERvA, NOvA and NuTeV), Dark Energy Survey, etc. Most significantly, the Fermilab's Tevatron remains the world's highest energy particle accelerator until the Large Hadron Collider becomes operational at CERN.

\subsection{The Fermilab Accelerator Complex}

The Tevatron accelerator at Fermilab is a circular synchrotron machine. Bunches of protons and antiprotons are accelerated in opposite directions and collide at $\sqrt{s}=$ $1.96 \mathrm{TeV}$. Initially, ionized hydrogen atoms $\left(H^{-1}\right)$ are accelerated in the CockroftWalton generator up to a kinetic energy of $750 \mathrm{KeV}$. The ions are injected into a linear accelerator (Linac) which accelerates their energy to $400 \mathrm{MeV}$. The electrons 
of the $H^{-1}$ ions are then stripped off by sending the ions through a carbon foil, which results in a $400 \mathrm{MeV}$ beam of protons. The $400 \mathrm{MeV}$ proton beam is sent into the Booster synchrotron and accelerated to an energy of $8 \mathrm{GeV}$. The accelerated beam of protons is finally sent into Main Injector synchrotron, and then into the Tevatron synchrotron. The Main Injector and Tevatron accelerate the protons to $150 \mathrm{GeV}$ and $980 \mathrm{GeV}$, respectively.

Antiprotons are produced by smashing a beam of protons with $120 \mathrm{GeV}$ energy from the Main Injector on to a rotating Nickel Target every $1.5 \mathrm{sec}$. The antiprotons along with other particles produced in the collisions, are produced off the target at many different angles. They are focused into a beam line with a Lithium lens. The beam following the Lithium lens contains several types of particles (e.g. pions, kaons, muons, positrons) in addition to antiprotons. Negatively charged particles are filtered by passing the beam through a pulsed magnet. The antiprotons are sent to the Debuncher ring to reduce the energy spread, and narrow the time of the beam. Pions and muons decay within $\sim 30$ rotations and electrons do not survive the first turn. The antiprotons are then cooled via electron cooling in the Recycler ring and accelerated so that they can be fed into the Main Injector. In the Main Injector, the proton and antiproton beams are accelerated up to the energy of $150 \mathrm{GeV}$.

Protons and antiprotons with an energy of $150 \mathrm{GeV}$ are separately injected into the Tevatron. The Tevatron ring accelerates the protons and antiprotons to a final energy of $980 \mathrm{GeV}$. The proton and antiproton beam are composed of 36 bunches each. There are three trains of 12 bunches with 396 ns separation circulating in the same beam pipe. The two beams are focused using quadrupole magnets at two points in the ring. The Tevatron has an array of $8 \mathrm{RF}$ cavities with the frequency of 53.03 
$\mathrm{MHz}$ which ramp the energy of beams to $980 \mathrm{GeV}$. Figure 2.1 shows the components of the Fermilab accelerator complex.

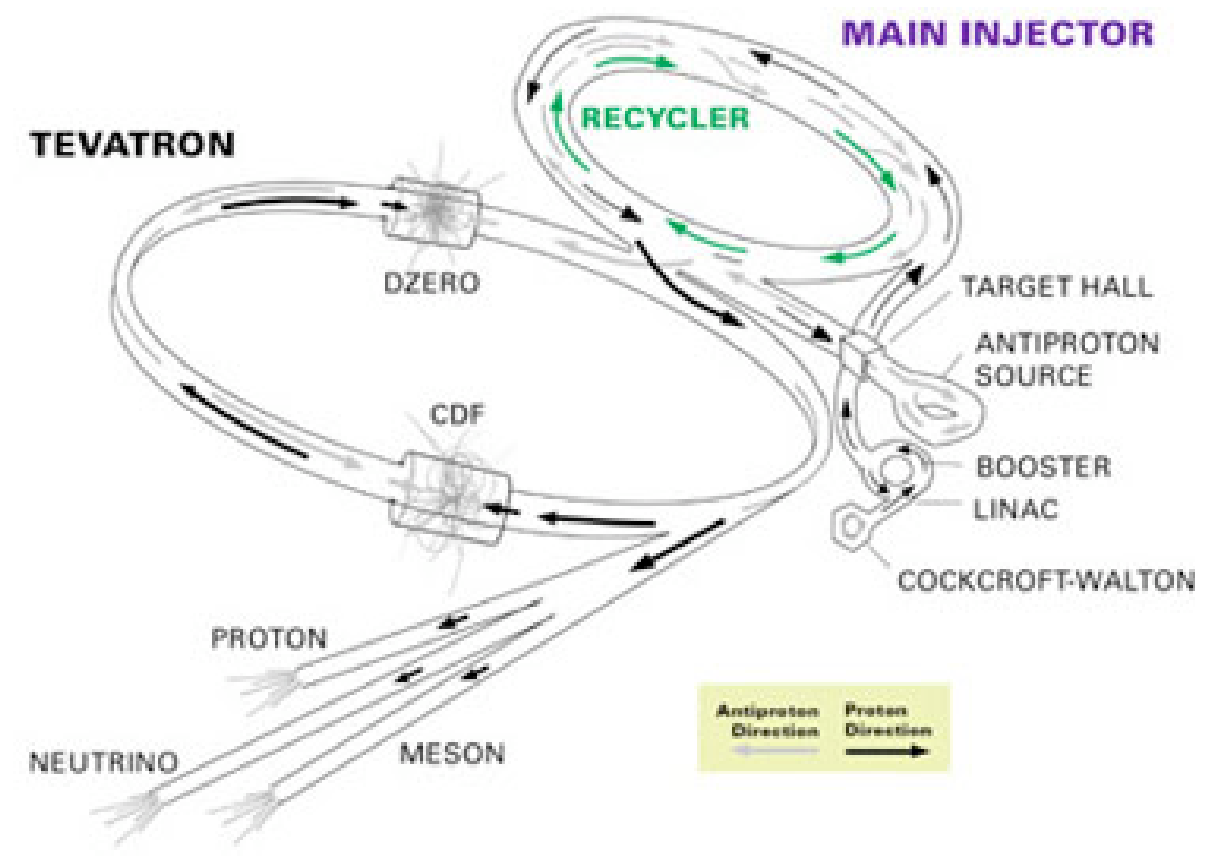

Figure 2.1: The plot shows the accelerator chain at Fermilab. Tevatron has two collision points, CDF and D0. At CDF, protons go in the $+\mathrm{z}$ (East) direction

\subsection{The Collider Detector at Fermilab}

The Collider Detector at Fermilab (CDF) is a complex but general purpose detector designed to investigate interesting events that are produced in $p \bar{p}$ collision at $\sqrt{s}=1.96 \mathrm{TeV}$. Collisions started in 1985, and data have been collected with a total integrated luminosity of $110 p b^{-1}$ in Run I. CDF Run II has been collecting data since 2001. As of middle of 2008, CDF has recorded data over $3 \mathrm{fb}^{-1}$ of integrated luminosity.

The CDF detector has a cylindrical geometry with axial and forward-backward 
symmetry. In the coordinate system of the CDF detector, the direction of the proton beam is defined as the $+z$ (East) direction. The $+x$ and $+y$ axis are chosen to be outward and upward from the Tevatron ring. The polar angle $\theta$ is measured from the $\mathrm{z}$ axis and the azimuthal angle $\phi$ is measured from the $+x$ axis. The pseudorapidity $\eta$ is defined in terms of the angle of a massless particle relative to the beam axis, $\eta=-\ln (\tan (\theta / 2))$.

The CDF detector includes tracking, calorimeter and muon chamber systems. The innermost part of the detector consists of the tracking system which is composed of a tracking silicon detector followed by a tracking open cell central drift chamber. The trackers are immersed in a 1.4 Tesla solenoidal magnetic field. The trajectories of electrically charged particle are measured, and the momentum and charge of particles are extracted from the bending of the tracks. Outside of the tracker, the calorimetric detectors are used to measure the energy of both charged and neutral particles. The calorimetric detectors are divided into two sections, a electromagnetic (EM) section for electrons and photons, followed by a hadron (HAD) section to measure the energy of charged and neutral hadrons. The outermost layers of CDF are muon chambers that track and identify muons. Although muons are unstable, their lifetime is long enough so that almost all of muons traverse the detector before decaying. Therefore, muons with sufficient energy penetrate the calorimeters and absorber steel and leave a track in the muon chambers. Figure 2.2 shows a half side view of the CDF Run II detector and Figure 2.3 shows the energy deposition of particles in each detector component. Photons and electrons deposit almost all of their energy in the EM calorimeter, and charged and neutral hadrons are mostly absorbed and deposit their energy in the HAD calorimeter. In general, muons do not interact in the calorimeter and reach 
the outer muon chambers. Neutrinos escape the detector without interacting and therefore are not directly measured in the detector. The transverse momentum of neutrinos is measured from the imbalance in the vector sum of the momentum of all other particles which are detected. In the analysis described here, the identification of electrons is the most important aspect. Electrons are fully reconstructed by the tracking and calorimetry system which are described in more detail in the following section.

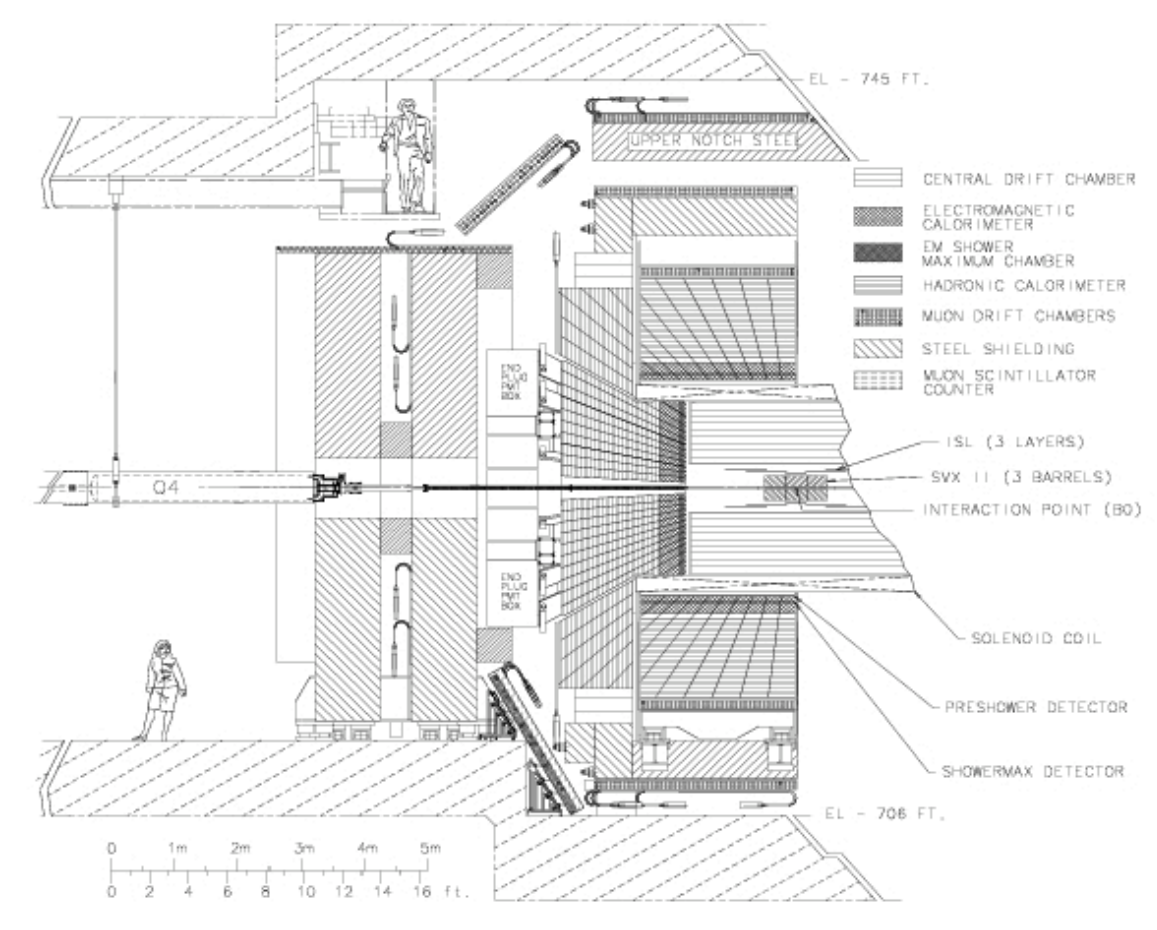

Figure 2.2: A half of the side view of the CDF Run II detector.

\subsubsection{The Silicon Tracker}

The silicon tracker is composed of layer 00 (L00) detector, the silicon vertex (SVX) detector, and the intermediate silicon layer (ISL) detector. Silicon layer 00 (L00) is a single sided silicon microstrip detector that improves the precision of the track 


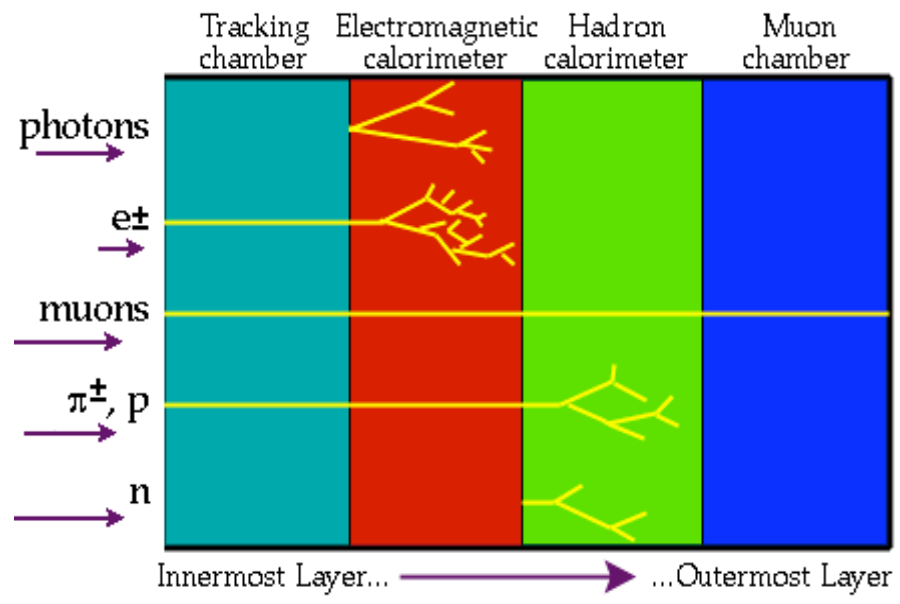

Figure 2.3: The components of the CDF the detector. The figure also illustrates how different types of particles interact in each detector component.

measurement and tagging efficiency for short lived particles. It is located immediately outside the beampipe, at a radius of approximately $\mathrm{r}=1.6 \mathrm{~cm}$ and covers $|\eta| \leq 4.0$. A total of seventy-two modules (six modules in $\mathrm{z}$ and twelve staggered wedges in $\phi$ ) compose L00. The six wedges in the $\mathrm{z}$ direction are narrow modules at a radius of $\mathrm{r}=1.35 \mathrm{~cm}$, and six more wedges are wide modules at a radius of $\mathrm{r}=1.62 \mathrm{~cm}$. The narrow and wide modules overlap for full tracking coverage. Figure 2.4 shows the end view of L00.

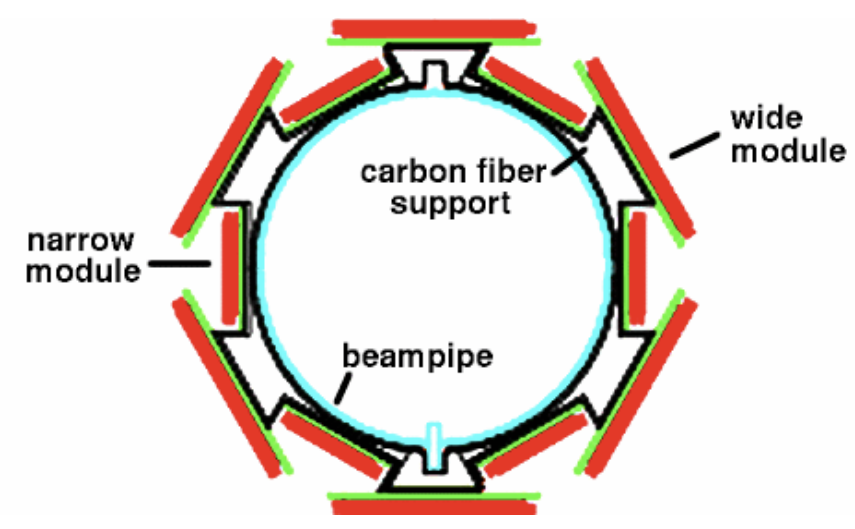

Figure 2.4: End view silicon Layer 00 (L00). 
The silicon vertex detector (SVX) is a double sided silicon microstrip detector located outside Layer00, extending from $\mathrm{r}=2.1 \mathrm{~cm}$ to $\mathrm{r}=17.3 \mathrm{~cm}$. It provides high precision tracking and secondary vertex detection at small radii. The SVX has 360 half-ladders organized into six bulkheads in $\mathrm{z}$, five layers in $\mathrm{r}$, and twelve wedges in the $\phi$ direction (as shown in Figure 2.5). The ladders in adjacent wedges overlap slightly to provide full coverage, and individual ladders get wider for successive layers. The innermost layer, layer-0, has 256 strips on the axial side of each ladder, and the outermost layer, layer-4 has 896 strips on the axial side of each ladder.

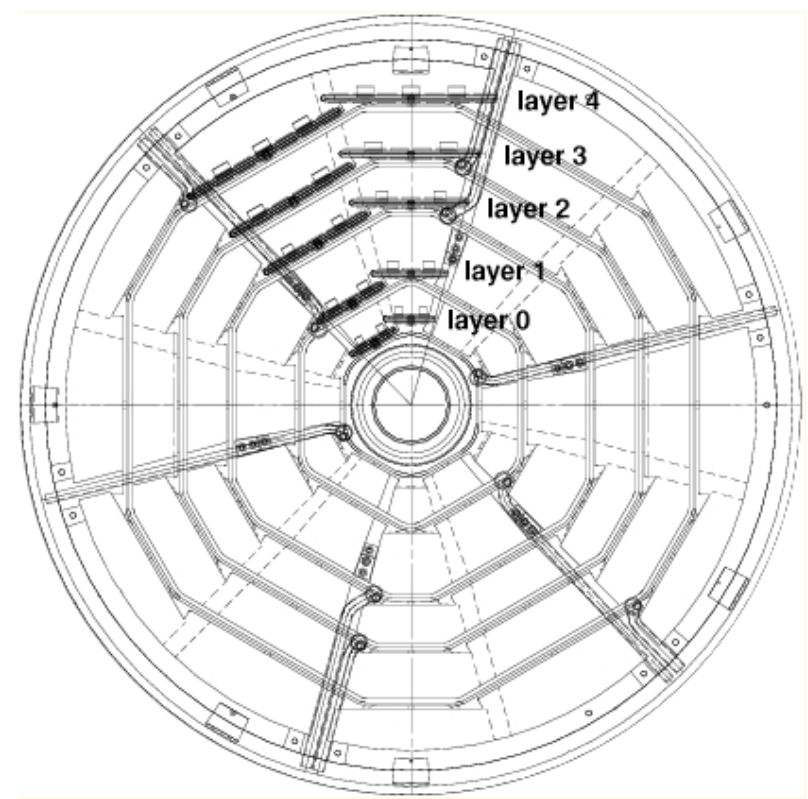

Figure 2.5: End view of SVX bulkhead. The SVX consists of five layers labeled as layer-0 to layer-4.

The intermediate silicon layer, ISL, is a double sided silicon microstrip detector similar to the SVX. It provides information to help the linking of tracks between the SVX and the central outer tracker (COT). It also provides improved silicononly tracking capabilities in the forward plug region, where the COT coverage is 
incomplete. The central layer is at $\mathrm{r}=22 \mathrm{~cm}$ and the forward and backward layers cover $1.0<|\eta|<2.0$. The central layer in the central barrel contains fifty-six ladders in two bulkheads and twenty-eight wedges in $\phi$. The two forward and backward layers in the two outer barrels contain 240 ladders in four bulkheads and twleve wedges in $\phi$.

\subsubsection{The Central Outer Tracker}

The Central Outer Tracker (COT) is a open cell drift chamber. A 50/50 argon-ethane gas mixture has a sufficiently high drift velocity to resolve beam crossings in conjunction with the small size of the drift cells. The COT is a general purpose tracking system in the central region that is used to measure charged tracks with transverse momentum $\left(p_{T}\right)$ as low as $400 \mathrm{MeV} / \mathrm{c}$. The 1.4 Tesla field of the CDF solenoid yields a momentum resolution $\delta p_{T} / p_{T}^{2} \leq 0.1 \% / G e V / c$. The pointing resolution of the COT is well matched to the silicon detector, so that charged tracks can be extrapolated and linked to the silicon hits with high efficiency. The COT consists of eight super-layers. Each super-layer consists of twelve sense wire layers. The super-layers alternate stereo and axial layers with a stereo angle of $\pm 3^{\circ}$. The active volume of the COT begins at a radius of $43 \mathrm{~cm}$ from the beamline and extends out to a radius of $133 \mathrm{~cm}$. The chamber is $310 \mathrm{~cm}$ long in the $\mathrm{z}$ direction. Table 2.1 provides a summary of the mechanical details of the COT.

Figure 2.6 and Figure 2.7 show the COT cell structure and a portion of one endplate. A single cell has twelve sense wires, 13 potential wires and four shaper wires in addition to a gold $(\mathrm{Au})$ on mylar field plane located on either side of the wire plane. The twenty-five sense plus potential wires are fabricated as a single unit, which allows for fast construction and pretesting of the planes. The COT has two 


\begin{tabular}{|l|r|}
\hline Number of Layers & 96 \\
Number of Super-layers & 8 \\
Material $\left(X_{0}\right)$ & $1.6 \%$ \\
Sense wire Spacing & $7.62 \mathrm{~mm} \mathrm{[0.3"]}$ \\
Wire Diameter & $40 \mu \mathrm{m}$ gold plated tungsten \\
Wire tension & $135 \mathrm{~g}$ \\
Drift Field & $1.9-2.5 \mathrm{kV} / \mathrm{cm}$ (depending on gas) \\
Maximum Drift Distance & $0.88 \mathrm{~cm}$ \\
Maximum Drift Time & $100 \mathrm{~ns}^{*}$ \\
Tilt Angle & $35^{\circ}$ \\
Length of Active Region & $310 \mathrm{~cm}$ \\
Total number of Wires & 73,080 \\
Endplate Load & 40 metric tons \\
\hline
\end{tabular}

\begin{tabular}{|l|cccccccc|}
\hline & \multicolumn{1}{|c}{ superlayer } \\
& 1 & 2 & 3 & 4 & 5 & 6 & 7 & 8 \\
\hline Stereo Angle (degrees) & +3 & 0 & -3 & 0 & +3 & 0 & -3 & 0 \\
Cells/Layer & 168 & 192 & 240 & 288 & 336 & 384 & 432 & 480 \\
Sense wires/Cell & 12 & 12 & 12 & 12 & 12 & 12 & 12 & 12 \\
Radius at Center of SL $(\mathrm{cm})$ & 47 & 59 & 70 & 82 & 94 & 106 & 117 & 129 \\
\hline
\end{tabular}

Table 2.1: A summary of the mechanical details of the COT[11]

aluminum endplates which carry the tension and provide much of the precision in the wire and field plane location. Wire planes and field sheets are strung between the two endplates. The endplates are machined with slots: one wire plane and one field sheet slot per cell per side. The precision machined edges of the slots determine the absolute location of the wire planes and field sheets.

The tracking system is crucial to electron identification. Electron candidates are reconstructed by matching the tracks in the tracking system to energy clusters in the electromagnetic calorimeter. The electron identification algorithms use the curvature and direction of the track. The curvature resolution is improved by constraining the 


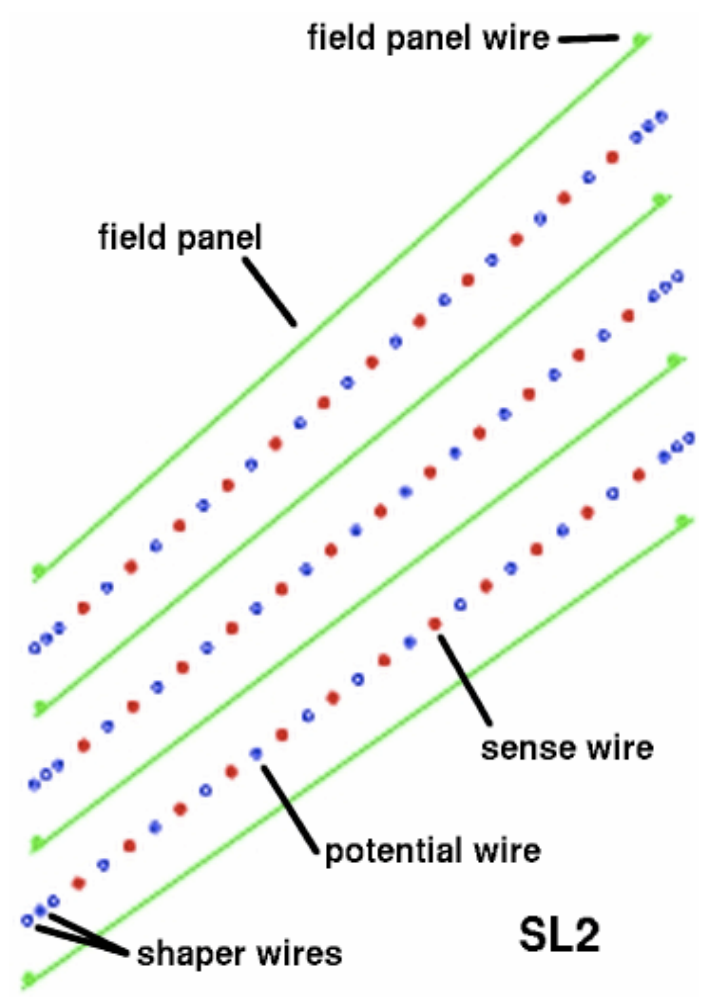

Figure 2.6: Three COT cells. Each cell has 12 sense wires, 13 potential wires, 4 shaper wires and a Au-mylar cathode field panel on both sides of the sense wire plane.

track to pass through the beam line.

\subsubsection{The Calorimeters}

The calorimeter system in the CDF detector is divided into two physical sections: the central and the forward end-plug region. The calorimeter in the central region $(\eta<1.1)$ is referred to as the "Central Calorimeter" and the calorimeter in the forward end plug region $(1.1<|\eta|<3.6)$ as the "Plug Calorimeter". Each calorimeter has electromagnetic (EM) and hadron (HAD) components to measure the energy deposition of charged and neutral particles. The EM calorimeter detects the energy of the electrons or photons, and the HAD calorimeter completes the measurement of 


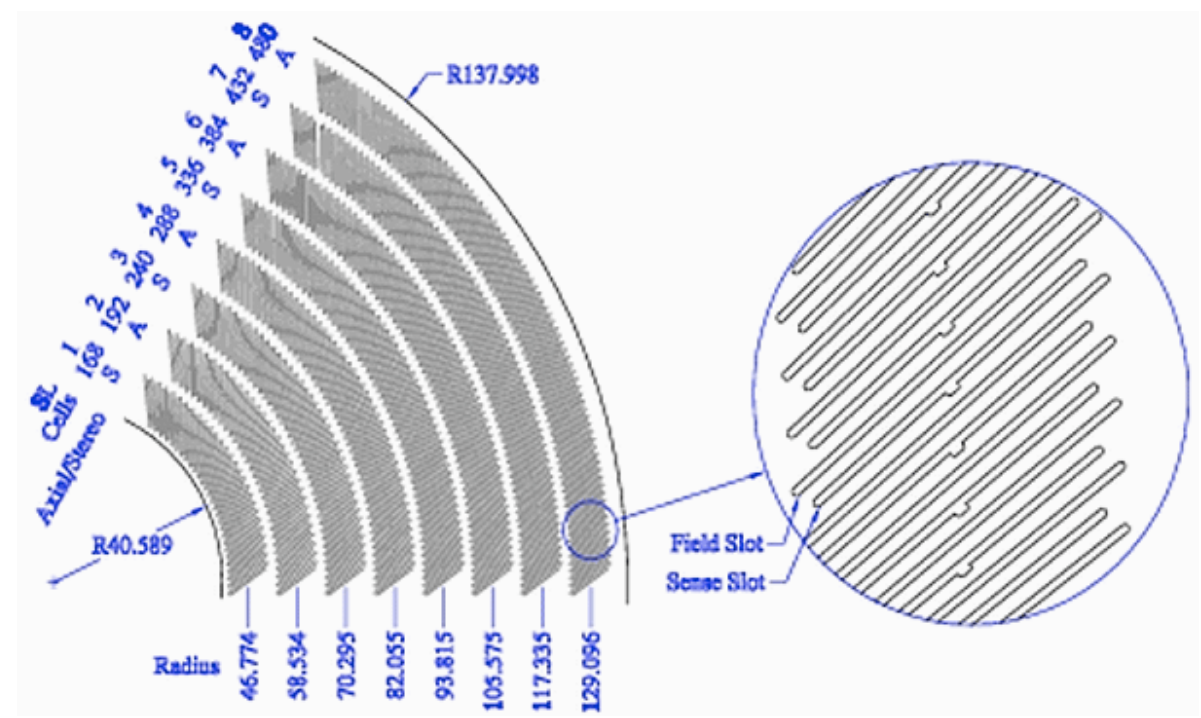

Figure 2.7: A view of a portion of one COT endplate. The slots define the radii of the eight superlayers. Within a superlayer, wire-plane slots and field sheet slots alternate.

hadronic showers.

\section{The Central Calorimeter}

The central calorimeter consists of the central electromagnetic (CEM), central hadronic (CHA), endwall hadronic (WHA) calorimeter, and central ShowerMax chambers (CES). The central electromagnetic calorimeter (CEM) is used to measure the energy of electromagnetic showers in the central detector region. The CEM is located just outside of the solenoid in the central part of the detector and covers $|\eta|<1.1$. The CEM is a 31 layer $\mathrm{Pb}$-scintillator sampling calorimeter. The layout of the CEM includes 478 projective towers in total which are organized into 24 wedges in $\phi$, and 10 tower groups in $\eta$ on each side. Towers $8 \mathrm{E}$ and $9 \mathrm{E}$ in wedge- 5 are removed to make room for the cryogenic utilities of the solenoid. Each tower is read out by two PMTs which are located in the $\phi$ direction of the tower. The resolution of the EM calorimeter, as measured in the test beam, is $13.5 \% / \sqrt{E_{T}}$. The central hadronic calorimeter 


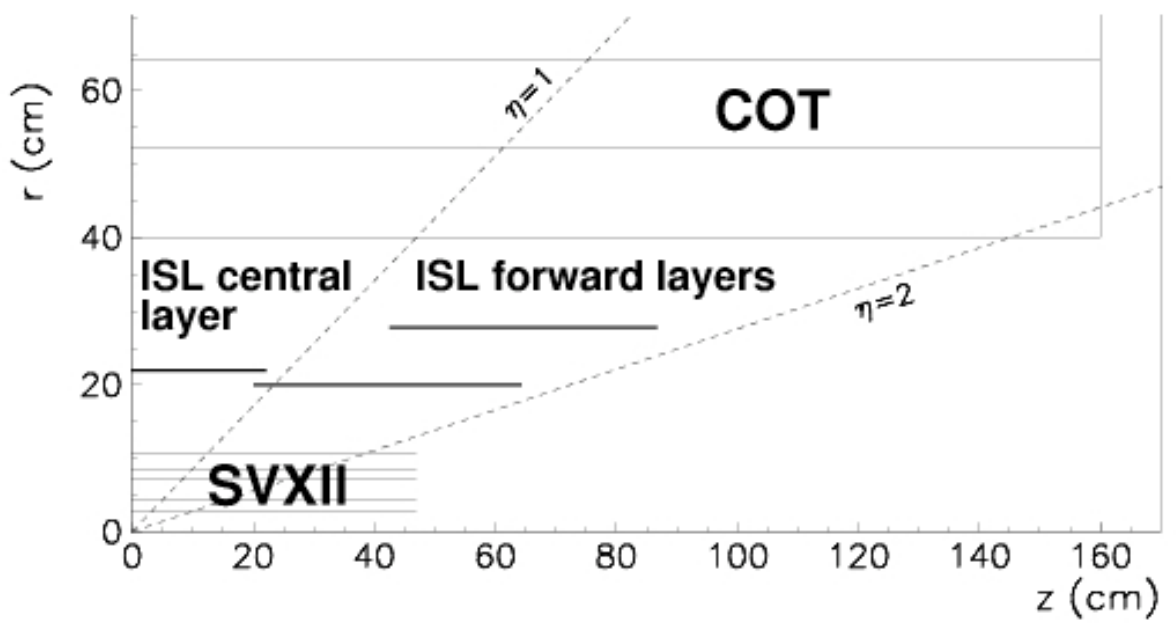

Figure 2.8: Aside view of the tracking system in $\mathrm{z}$ and $\mathrm{r}$ direction.

(CHA) is located outside of the CEM and measures the energy of hadron showers in the central $|\eta|<0.9$ region. The $\mathrm{CHA}$ is a steel-scintillator sampling calorimeter consisting of twenty-four wedges in $\phi$ and 8 tower groups in eta on each side. The cryogenic utilities of the solenoid (chimney) also run through wedge 5E of the CHA. The endwall hadronic calorimeter (WHA) is a steel-scintillator sampling calorimeter and extends the hadronic calorimeter coverage to $|\eta|=1.3$. It is organized into twenty-four wedges in $\phi$ and 6 tower groups in $\eta$. The central ShowerMax chamber consists of the central EM ShowerMax (CES), the central Pre-Radiate (CPR), and the central crack (CCR) chamber. The CES is a strip detector used to measure shower centroids, and is at a depth of $\sim 6$ radiation lengths. The CPR is a central pre-radiator which is behind the solenoid and just in front of CEM. The CCR is a crack detector, at same radius as CPR but at the $\phi$ position of the steel between wedges. It is used to measure the energy in those inter-tower $\phi$ cracks. These detector components provide additional track linking ability and transverse shower profiles to improve particle identification and $\pi^{0} / \gamma$ separation. Figure 2.9 shows details of a 
wedge of the central calorimeter.

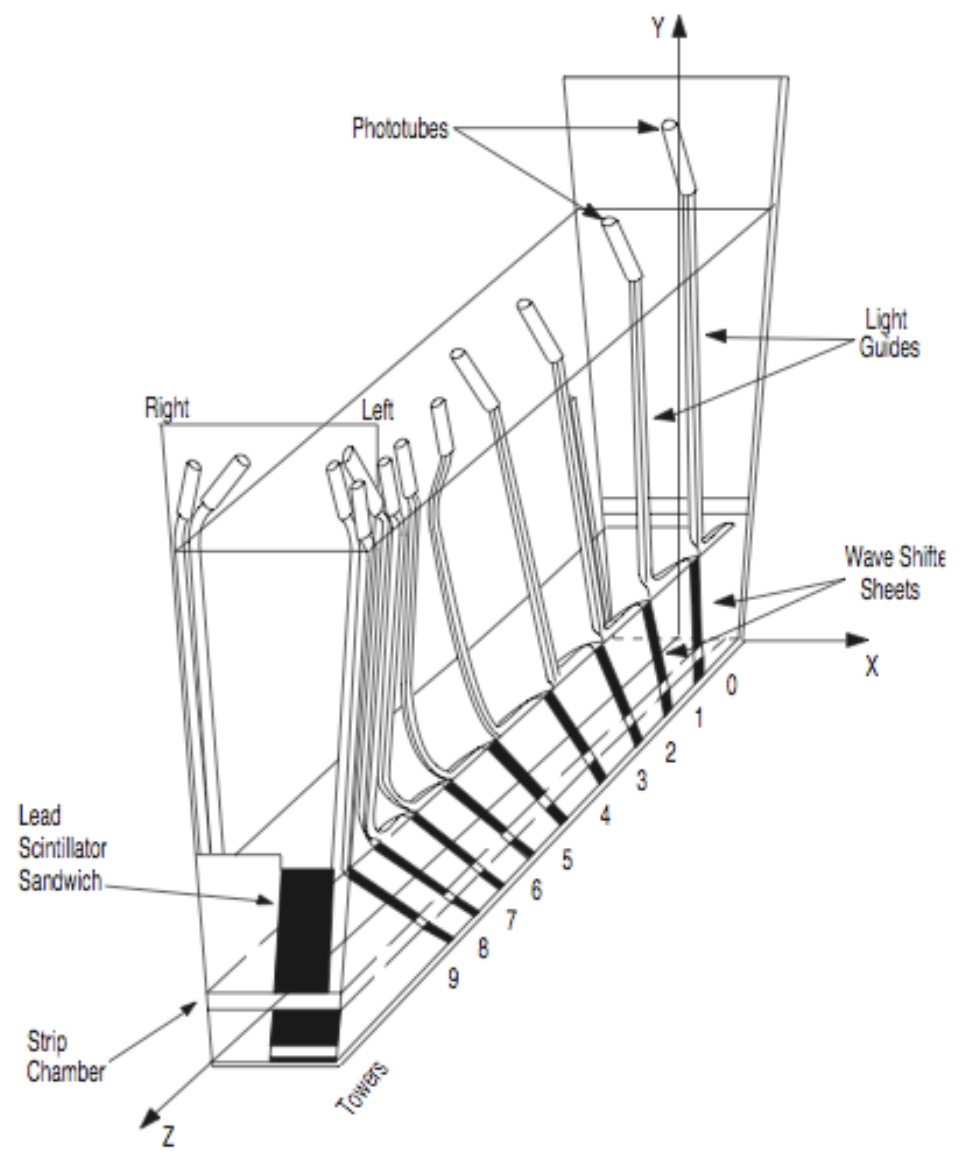

Figure 2.9: A wedge of the central calorimeter. Each wedge covers one tower in the $\phi$ direction $\left(\Delta \phi=15^{\circ}\right)$ and ten towers in the $\eta$ direction.

\section{The End Plug Calorimeter}

The end plug calorimeter consists of the plug electromagnetic (PEM), plug hadronic (PHA), and plug Shower-Max and Pre-Radiator detectors (PES and PPR). The plug electromagnetic calorimeter (PEM) is a Pb-scintillator sampling calorimeter like the CEM, and is used to measure the energy of the electromagnetic showers in the forward region, $1.1<|\eta|<3.6$. The plug hadron calorimeter (PHA) is a steel-scintillator 
sampling calorimeter with the same coverage as the PEM and is used to measure the energy of the hadronic showers. The upgraded end plug calorimeters (PEM and PHA) are new for Run II (the construction of the PHA was directed by the University of Rochester). The upgraded scintillator-based calorimeters replace the Run I gas-based calorimeters previously used in the plug region to obtain faster timing and better energy resolution. The PEM energy resolution is about $\sigma / E=$ $17 \% / \sqrt{E} \oplus 1 \%$, and the PHA hadron resolution is about $\sigma / E=80 \% / \sqrt{E} \oplus 5 \%$. The upgraded end plug calorimeter add additional coverage in theta which is extended to 3 degrees (versus 10 degrees in Run I). The PEM is organized in 12 tower groups in $\eta$. The arrangement of the PHA is the same as the PEM except the outermost tower group because of the detector geometry. Details are shown in Figure 2.10. The plug electromagnetic Shower-Max detector (PES) is a scintillator strip detector embedded within the PEM at a location approximately six radiation lengths deep. It provides precision measurements of the transverse positions of the shower and yields improved ability to separate electrons, pions, and photons. The PES is divided into eight 45-degree wedges per plug with two layers of 200 strips each. In one layer, the strips are oriented parallel to one side of the wedge and in the other layer, parallel to the other side. In each wedge, the strips are divided into upper $(1.13<\eta<2.60)$ and lower $(2.60<\eta<3.50)$ regions in $\eta$. [12] The plug pre-radiator detector (PPR) is constructed of scintillator tiles located in the first scintillator layer of the PEM. The PPR provides enhanced ability to discriminate between electrons and pions. Figure 2.11 shows the tile-fiber component in the plug calorimeter. 


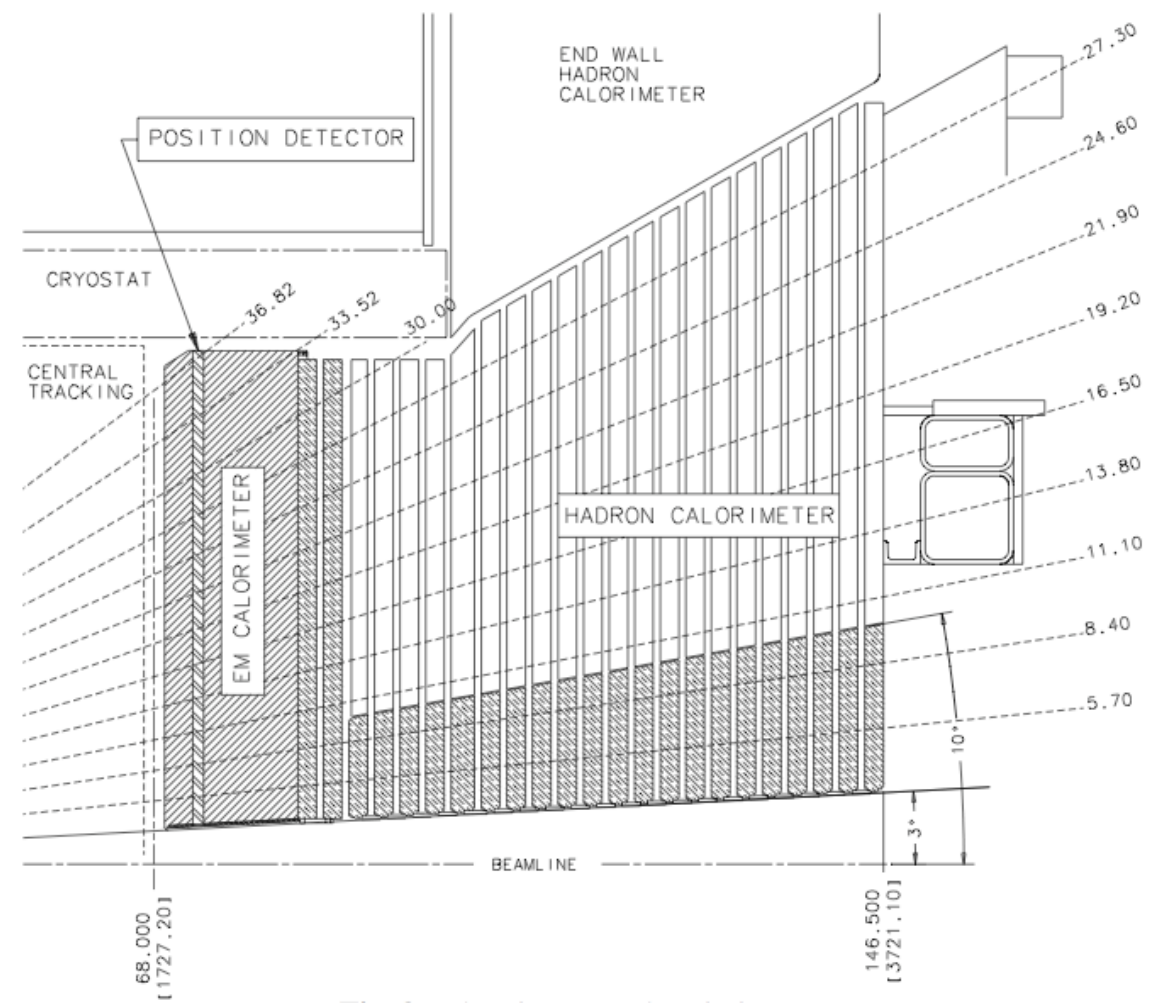

Figure 2.10: A side view of the plug calorimeter (PEM, PHA).

\subsection{The Trigger System}

CDF has a three-level trigger to select interesting events out of a $1.7 \mathrm{MHz}$ crossing rate. It collects events to magnetic tape at an average rate of $120 \mathrm{~Hz}$. Figure 2.12 shows the data flow of the CDF trigger system.

\subsubsection{Level-1}

The Level-1 (L1) trigger is a synchronous system with an event read in every beam crossing and a trigger decision made every beam crossing. This is the first stage of the trigger system and provides a large reduction in event rate. The L1 trigger 


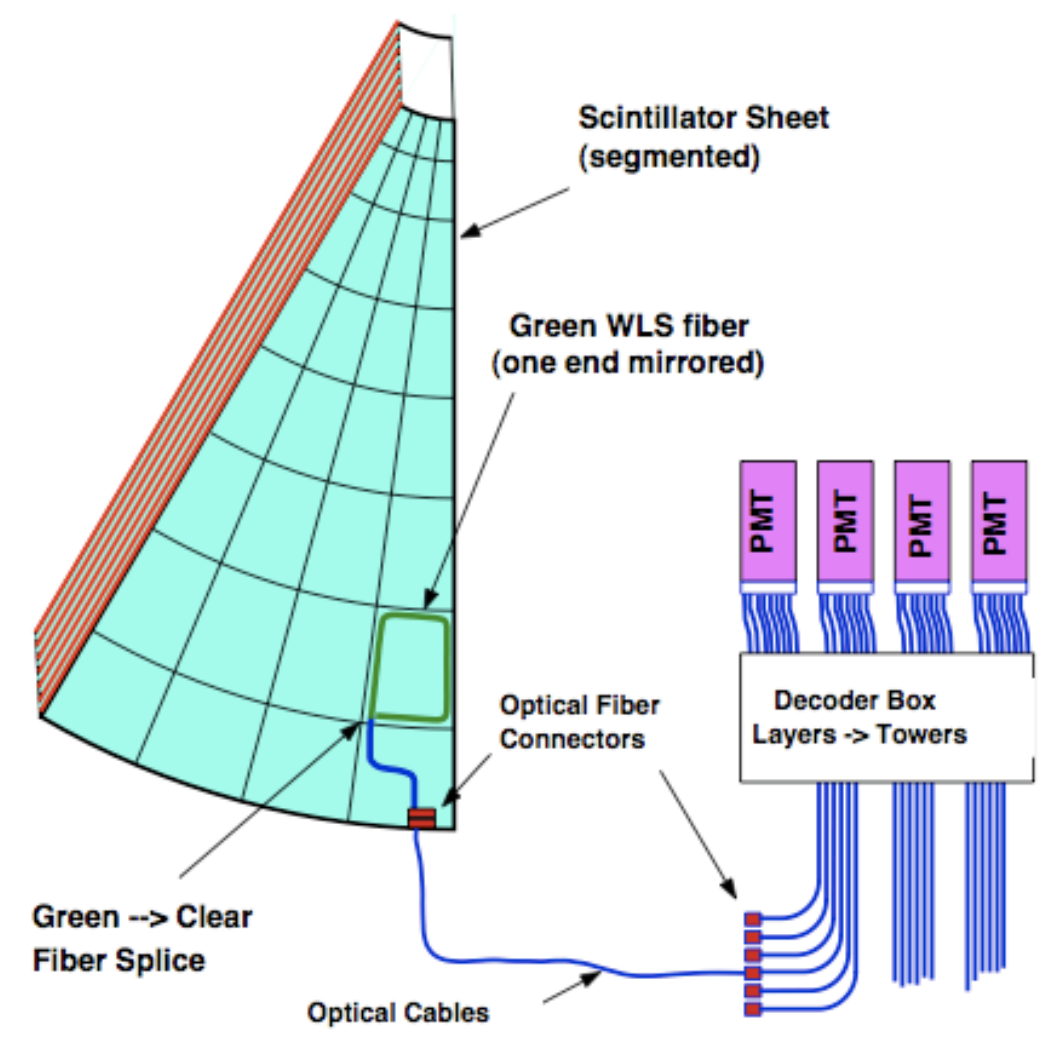

Figure 2.11: A scintillator tile-fiber component in the plug calorimeter.

decision occurs at a fixed time which is $\sim 5 \mu s$ after the beam crossing. The input rate of $\mathrm{L} 1$ is $1.7 \mathrm{MHz}$ and the acceptance rate is $\sim 20 \mathrm{KHz}$ limited by L2. The L1 trigger has various trigger primitives to reduce the acceptance rate. In L1, three parallel synchronous streams are processed to reconstruct the COT tracks, the energy depositions in the calorimeter tower, and the muon tracks in the muon chamber. The COT track is matched to the calorimeter and muon chamber information. The information from these streams is sent to the global level-1 decision to select events based on the each trigger requirement. Events which satisfy the trigger requirement are accepted and sent to the second stage trigger, Level-2. 


\subsubsection{Level-2}

The Level-2 (L2) trigger is a combined hardware and software trigger. The average processing time of L2 is $\sim 30 \mu s$ and the acceptance rate is $\sim 480 \mathrm{~Hz}$. The L2 trigger also has several asynchronous subsystems to collect the L1 information and perform additional reconstruction. In L2, the displaced vertex of the track, energy cluster information from the calorimeter, and the EM ShowerMax information are used to identify particles. The reconstructed information is sent to the global level-2 decision to select events which satisfy the L2 trigger requirement. Figure 2.13 shows the various trigger subsystems of L1 and L2.

The L2 trigger was upgraded in 2006 to improve its capability for the increasing instantaneous luminosity. The main upgrade has been on L2cal, which is the energy clustering in L2 using Pulsar boards. The Pulsar board provides a sufficient safety margin in bandwidth and additional usage flexibility. Therefore, the upgraded L2cal boards provide the full calorimeter trigger tower information directly to the L2 decision. This allows for more sophisticated algorithms for EM clustering, L2 jets, and MET with the full resolution of the trigger tower information. The upgraded trigger based on the Pulsar boards is also used by the L2 global decision crate and the L2 silicon vertex tracking (SVT) subsystem.

The L2 upgrade provides a higher purity data sample and improves the sensitivity for searches for new physics by providing more complete information for jet and MET related triggers. 


\subsubsection{Level-3}

The last stage in the trigger system is Level-3 (L3) which is purely a software trigger using a massive PC computer farm. The acceptance rate of L3 on average, is about $120 \mathrm{~Hz}$. The main subsystems of L3 are the Event Builder (EVB) and the L3 Farm. The Event Builder assembles all event fragments from the Front End crates into one data block. The L3 Farm takes these events and reconstructs the events for optimized analysis. The final selection for each of the physics processes of interest is decided in this stage. The L3 trigger uses the full detector information to reconstruct particles, so it provides better reduction than any other lower trigger levels. The events selected at L3 are then passed to the Data-Logger subsystem. 


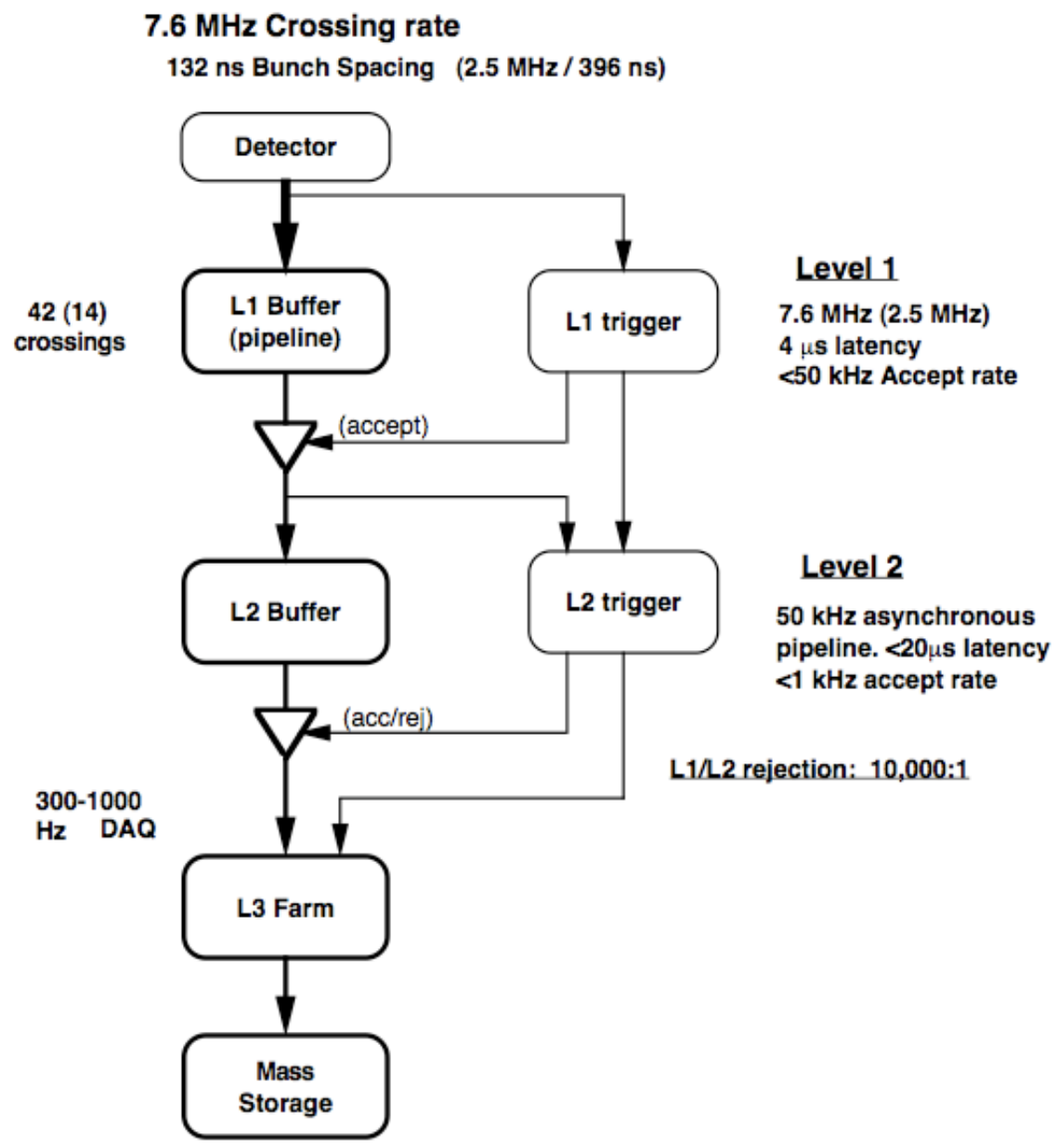

Figure 2.12: The trigger system of the CDF detector. The CDF trigger has a threelevel system (L1, L2, and L3) to select events. 


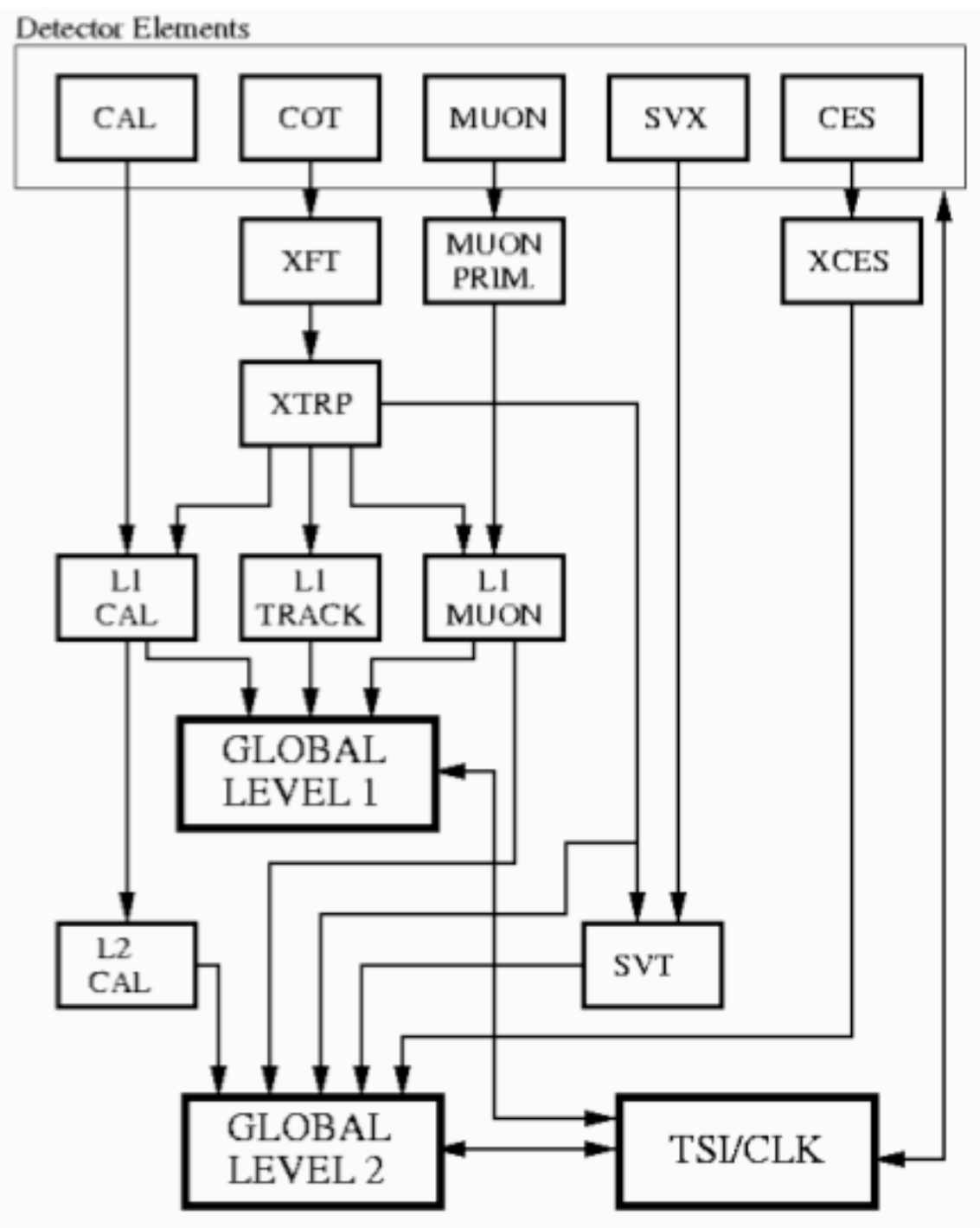

Figure 2.13: The L1 and L2 trigger subsystems. L1 uses COT tracking, calorimeter, and muon track information and L2 uses SVT (displaced vertex), energy cluster, and ShowerMax information for the event selection. 


\section{Chapter 3}

\section{Data}

\subsection{Data Sample}

The analysis uses data which were collected from February, 2002 to May, 2007. Good runs, i.e. runs with no detector malfunction during operation, are required. After selecting good runs, the total integrated luminosity for $\mathrm{Z}$ events with two central electrons or one central and one plug electron is $2128.1 p b^{-1}$. For Z events with two plug electrons, the integrated luminosity is $2020.3 \mathrm{pb}^{-1}$ because of the silicon track requirement for one of the two plug electrons. The events with two electrons are required to satisfy either " $E L E C T R O N_{-} C E N T R A L \_18$ ", or the " $Z_{-} N O T R A C K$ " trigger path. The $Z_{-} N O T R A C K$ trigger path was implemented after Run number is 143938. Therefore, the integrated luminosity includes Z events after Run 143938.

\subsection{Monte Carlo Sample}

Monte Carlo (MC) sample are used to study the detector acceptance and $\mathrm{Z}$ event selection efficiency. The MC sample is produced using the PYTHIA [15] event generator, and a GEANT detector simulation. The PYTHIA generator includes the effects of initial and final state QCD and QED radiation in conjunction with a parton 
shower algorithm. The events are generated with the CTEQ5L parton distribution functions (PDFs) which is LO PDFs provided by CTEQ collaboration. The Monte Carlo is tuned to describe the underlying event, and the $P_{T}$ spectrum of $\mathrm{Z}$ bosons in agreement with measured data. The detector simulation models the decays of generated particles and their interactions with the detector components. Interactions with material in the detector volume result in bremsstrahlung processes as particles traverse the detector. The amount of material before the calorimeter is tuned such that the simulation of electrons is consistent with the observed data. The energy scale of electrons in the MC sample is tuned by comparing the $E_{T}$ distribution of electrons, and the position of the $\mathrm{Z}$ mass peak for data and MC events. A total of 20.3 million $\mathrm{MC}$ events generated with the Drell-Yan $Z / \gamma^{*} \rightarrow e^{+} e^{-}$process are used in the analysis. In addition, samples of $W+$ jet, $W W / W Z$, and $t \bar{t} \mathrm{MC}$ events are used for background studies.

\subsection{Electron Candidates}

\subsubsection{Electron Identification}

A central electron is defined as an electromagnetic (EM) object found in the region $|\eta|<1.1$. An EM object is reconstructed by clustering of energy depositions in the EM calorimeter. The first step in the selection of an EM object cluster is to find the seed tower which has the highest $E_{T}$ with $E_{T}>2 \mathrm{GeV}$. Here $E_{T}$ is the transverse component of energy measured in the EM calorimeter $\left(E_{T}=E \times \sin \theta\right)$. In the clustering process, $E_{T}$ is calculated assuming the event originates from the center of the detector, $z=0$. The adjacent towers on either side of $\eta_{\text {Det }}$ are added to the seed tower as shoulder towers for $E_{T}>100 \mathrm{MeV}$. ( $\eta_{\text {Det }}$ is the pseudorapidity of an 
electron assuming that $p \bar{p}$ interaction point along the $\mathrm{z}$ direction of the beam line is in the center of the detector.) A plug electron is defined as an EM object found in the region $|\eta|>1.1$. In clustering the energy in the plug region, the energy deposition in a nine tower, $3 \times 3$ geometry around the seed tower is used. The calorimeter response depends on the transverse location of the energy deposition in the tower. This dependence is corrected for after the clustering using information from test beam data. The energy response to electrons entering at the different points in the tower has been measured in a test beam and this information is used to correct for the spatial dependence of the response. The electron is a charged particle which is tracked by the tracking system. The COT tracker covers all of the central region and is very efficient $(\sim 96 \%)$. The tracking information provides the vertex position of events, and a track matched to the EM object allows for better electron identification. The information from the ShowerMax (CES) detector is also used for improved electron identification. The CES consists of sense wire and cathode strips in the perpendicular direction in the $z-\phi$ coordinate system. It provides a measurement of the position of an electron in the calorimeter with high resolution.

Electron candidates are selected by applying electron identification selection criteria as described below.

\section{- $E_{T}$}

The transverse component of the electron energy, $E_{T}$ is defined as $E_{T}=E \times$ $\sin \theta$. Here, $E$ is the electron energy deposited in the calorimeter cluster after energy response corrections. The polar angle with respect to the beam line $(\theta)$ is corrected using the vertex information of the matched track. For plug electrons, the energy loss measured in the PPR detector is added to the electron energy. 
Finally, the corrected $E_{T}$ is required to be above the selection threshold.

\section{- Track Fiduciality}

The Track Fiduciality requirement ensures that the electron pass the instrumented active region of the detector. For the central electron, the COT track position is required to be in the region $9<\mid$ Track $z \mid<217 \mathrm{~cm}$ and $\mid$ Track $x \mid<$ $21 \mathrm{~cm}$. If the track is found to point to tower 5 in east side $(5 \mathrm{E})$ or tower 9 , the event is rejected. Tower $5 \mathrm{E}$ is the Chimney (where cables and cryogenic pipes are located), and tower 9 is located at the the end of the central calorimeter, and does not have full calorimeter coverage in $\eta$. Therefore, full reconstruction in these regions cannot be made.

\section{- $\operatorname{Track} z_{0}$}

The Track $z_{0}$ is the interaction point along the $\mathrm{z}$ direction of the beam line from which the electron originates. It is obtained from the track matching the electromagnetic cluster. The position is required to be within $60 \mathrm{~cm}$ from the center of the detector, in order to ensure that the trajectory of the particle is in the tracking volume.

\section{- $P_{T}$}

The $P_{T}$ is the transverse component of the momentum of the track matched to the electron object. This variable is required to be be greater than the $P_{T}$ threshold for electron selection.

\section{- $\mathrm{Had} / \mathrm{Em}$}

The $\mathrm{Had} / \mathrm{Em}$ ratio is the fraction of the energy deposited in the HAD and the EM calorimeter. A real electron deposits most of its the energy in the EM 
calorimeter. On the other hand, hadrons and hadronic jets deposit most of the energy in the HAD calorimeter, and only a small fraction of the energy in the EM calorimeter. The $\mathrm{Had} / \mathrm{Em}$ ratio allows us to distinguish electrons from hadronic particles by requiring $\mathrm{Had} / \mathrm{Em}$ to be smaller than a selected cut value.

\section{- Isolation}

The Isolation is defined as the energy contained in the cone within a region $\Delta R\left(\sqrt{\Delta \phi^{2}+\Delta \eta^{2}}\right)<0.4$ around the electron (after subtracting the energy of the electron itself). The Isolation energy of real electrons originating from $W$ or $Z$ decays is very small because no associated hadrons are produced in these decays. On the other hand, electrons from the decay of bottom and charm quarks are typically produced in association with other hadrons, either from the original heavy quark decays or from the associated QCD jet. Similarly, hadronic particles in a QCD jet, which may be misidentified as electrons, are associated with other hadrons in the hadronic shower. These other hadrons or neutral pions in the jet contribute to a higher Isolation energy. We require an electron to have an isolation energy below the Isolation energy threshold.

\section{- COT Quality}

The COT track quality requirement selects electrons with a good track as reconstructed by the COT tracker. To reconstruct a good track, we require that the track contains super layers with at least 5 hits. The number of super layers with more than 5 hits is required to be greater than the cut value for electron selection. 
- $E / P$

The $E / P$ ratio is the ratio of the transverse energy of the electron cluster measured in the EM calorimeter to the transverse momentum of the track measured in the COT. In the high energy limit, an electron can be regarded to be massless and the energy, $E$, would be equal to the magnitude of the momentum, $P$. This implies that for electrons $E / P=1$. However, electrons sometimes radiate photons when passing through the detector material. Most of the photons are emitted colinearly and end up in the same tower as the electron, However, the electron itself has lost energy, ending with a track of lower $P_{T}$. Therefore, in general the $E / P$ for electrons is expected to be greater than 1.0. Any electron can also have a low $E / P$ when $P$ is mis-measured, especially when the momentum is high and the track curvature is small. For electron selection, the $E / P$ is required to be less than the selection cut value.

- $\Delta z$ and $Q \times \Delta x$

The track associated with the electron is extrapolated to the CES plane, and the extrapolated position coordinates are compared to the position measured in the ShowerMax detector. Here $\Delta z$ is defined as the separation in the $\mathrm{z}$ direction between the extrapolated track position and the CES cluster. The variable, $\Delta x$ is the corresponding separation in the $r-\phi$ direction, and $\mathrm{Q}$ is the charge of the track. The $\Delta z$ and $Q \times \Delta x$ variables are used to associate tracks with EM clusters.

- $L_{s h r}$

The variable $L_{s h r}$ is a measure of the lateral shower profile for electrons. It compares the energy distribution in CEM towers adjacent to the seed tower for 
electrons in data to the distribution expected from measurements of electrons in the test beam.

- $\chi_{\text {strip }}^{2}$

The $\chi_{\text {strip }}^{2}$ is a $\chi^{2}$ comparison of the CES shower profile in the r-z dimension to the expected profile as measured for electrons in the test beam. The $\chi_{\text {strip }}^{2}$ is required to be less than the selection cut value.

\section{- Fiducial $\eta$}

For plug electrons, the $\eta_{\text {Det }}$ range is used as a cut variable to determine fiduciality for plug electrons. The $\eta_{D e t}$ from the best matching 2-dimensional PES cluster is used when an electron has the PES cluster. If an electron fails to have the PES cluster, the $\eta_{\text {Det }}$ from the $3 \times 3$ PEM cluster is used.

\section{- Silicon track fiduciality}

A silicon track can be reconstructed from three and more silicon hits. Therefore, the expected number of silicon layers which the electron passes through is required to be greater than three assuming that there are no multiple hits in one layer. The expected number of silicon layers that the electron passes through is calculated using the silicon geometry, the vertex of the electron in the z-direction, and the PES position of the electron. The silicon track fiduciality requirement is that the expected number of silicon layers traversed by the electron track is more than three and that the electron has a reconstructed $\mathrm{z}$ vertex.

\section{- Phoenix Tracking}

The phoenix tracking is the forward electron tracking algorithm. In the high 
$\eta_{\text {Det }}$ region, silicon stand-alone tracking is very difficult because there are only a few layers of silicon available for the track to pass through. The phoenix tracking algorithm uses additional information to help constrain to the silicon hits and improve the tracking efficiency in the forward region. It creates the predicted track using the primary vertex and the shower-max hit position. Silicon hits, if found within a certain window about the trajectory of the predicted track, are added to complete the reconstruction of the track. For the phoenix tracking requirement, the number of the silicon hits found is required to be greater than 3 and the track $z_{0}$ should be within $60 \mathrm{~cm}$ of the center of the detector.

\section{- $P E M \chi_{3 \times 3}^{2}$}

The variable, $P E M \chi_{3 \times 3}^{2}$ is the $\chi^{2}$ value of the $3 \times 3$ PEM cluster energy distribution as compared to the hypothesis that EM object is an electron cluster. The electron selection requires this variable to be less than the selection cut value.

Table 3.1 and 3.2 show the requirements for central and plug electrons, respectively.

\subsection{Z Event Selection}

Only events reconstructed with two electron candidates are used in this analysis. These include event topologies with two central electrons(CC), one central and one plug electron(CP), and two plug electrons $(\mathrm{PP})$.

The $\mathrm{Z}(\mathrm{CC})$ sample consists of $\mathrm{Z}$ events reconstructed with one tight and one loose central electron. In the $\mathrm{Z}(\mathrm{CC})$ topology, $\mathrm{Z}$ events with opposite electric charge electrons are selected. The $\mathrm{Z}(\mathrm{CP})$ sample consists of $\mathrm{Z}$ events with one tight central 


\begin{tabular}{|c|c|c|}
\hline Variable & Tight central & Loose central \\
\hline$E_{t}$ & $>25(C C), 20(\mathrm{CP}) \mathrm{GeV}$ & $>25 \mathrm{GeV}$ \\
Fiducial & Track fiduciality & Track fiduciality \\
Track $z_{0}$ & $\mid$ Track $z_{0} \mid<60$ & $\mid$ Track $z_{0} \mid<60$ \\
$P_{T}$ & $>10 \mathrm{GeV}$ & $>10 \mathrm{GeV}$ \\
\hline Had $/$ Em & $<0.055+0.00045 \times E$ & $<4.0$ \\
IsolationE & $<4.0$ & \\
$E / P$ & $E / P<2.0$ or $P_{T}>50$ & \\
COT quality & Axial $S L s$ with5 hits $/ S L>3$ & \\
& Stereo $S L s$ with 5 hits $/ S L>2$ & \\
$L_{\text {shr }}$ & $<0.2$ & Stereo SLs with Shits $/ S L>2$ \\
$\chi_{\text {strip }}^{2}$ & $<10$ & \\
$Q \times \Delta x$ & $-3.0<Q \times \Delta x<1.5$ & \\
$|\Delta z|$ & $<3.0$ & \\
\hline
\end{tabular}

Table 3.1: central electron selection cuts

and one plug electron. The $\mathrm{Z}(\mathrm{PP})$ sample consists of $\mathrm{Z}$ events with two plug electrons, for which one of them is required to have a track in the silicon tacker (phoenix track ) in order to reduce the background contamination. Since the forward region is close to the beam line, the backgrounds from associated jet processes and the underlying event are larger.

The forward region is important in the measurement because it is associated with high rapidity $\mathrm{y}$, and provides information about PDFs at high $\mathrm{x}$. The silicon phoenix tracking was developed especially for electron identification in the forward region. We include plug-plug events in order to increase the acceptance at large $y$, and as stated earlier, for PP events we require that one of two electrons has a silicon phoenix track in order to reduce the background from QCD jets. Only Z(PP) events with two electrons found in the same detector side are selected. This criteria rejects dijet events with glancing collisions, where one jet ends up in one plug and the other jet ends up 


\begin{tabular}{|c|c|c|}
\hline Variable & Phoenix electron & Plug electron \\
\hline$E_{t}$ & $>25 \mathrm{GeV}$ & $>20(\mathrm{CP}), 25(\mathrm{PP}) \mathrm{GeV}$ \\
Fiducial & $1.2<\left|\eta_{\text {Det }}\right|<2.8$ & \\
silicon track fiduciality & expected silicon hit $>=3$ & \\
z vertex & any reconstructed z vertex & \\
\hline Had/Em & $<0.05$ & $<0.05$ \\
IsolationE & $<4.0$ & $<4.0$ \\
PEM $\chi_{3 \times 3}^{2}$ & $<10$ & $<10$ \\
Phoenix SiHit number & $\geq 3$ & \\
Phoenix Track $z_{0}$ & $\mid$ Track $z_{0} \mid<60$ & \\
\hline
\end{tabular}

Table 3.2: Plug electron selection cuts

in the other plug.

Using the above selection criteria, we find $50784 \mathrm{Z}(\mathrm{CC})$ events, $86230 \mathrm{Z}(\mathrm{CP})$ events, and $31346 \mathrm{Z}(\mathrm{PP})$ events in the invariant mass window, $66<M_{e e}<116 \mathrm{GeV} / \mathrm{c}^{2}$. Table 3.6 summarizes the number of events in boson rapidity after selection in each topology.

The mean and width of a Gaussian fit to the invariant mass distributions in the peak region $86<M_{e e}<96 \mathrm{GeV} / \mathrm{c}^{2}$ (adding the PPR Energy for plug electron) is 90.60 $\mathrm{GeV}$ with a width of $2.92 \mathrm{GeV}$ for $\mathrm{Z}(\mathrm{CC})$ events , $90.53 \mathrm{GeV}$ with a width of 3.12 $\mathrm{GeV}$ for $\mathrm{Z}(\mathrm{CP})$ events, and $90.26 \mathrm{GeV}$ with a width 3.22 for $\mathrm{Z}(\mathrm{PP})$ events as shown in Table 3.3 .

Figure 3.1 shows the invariant mass and rapidity distribution in each of the three event topologies. 

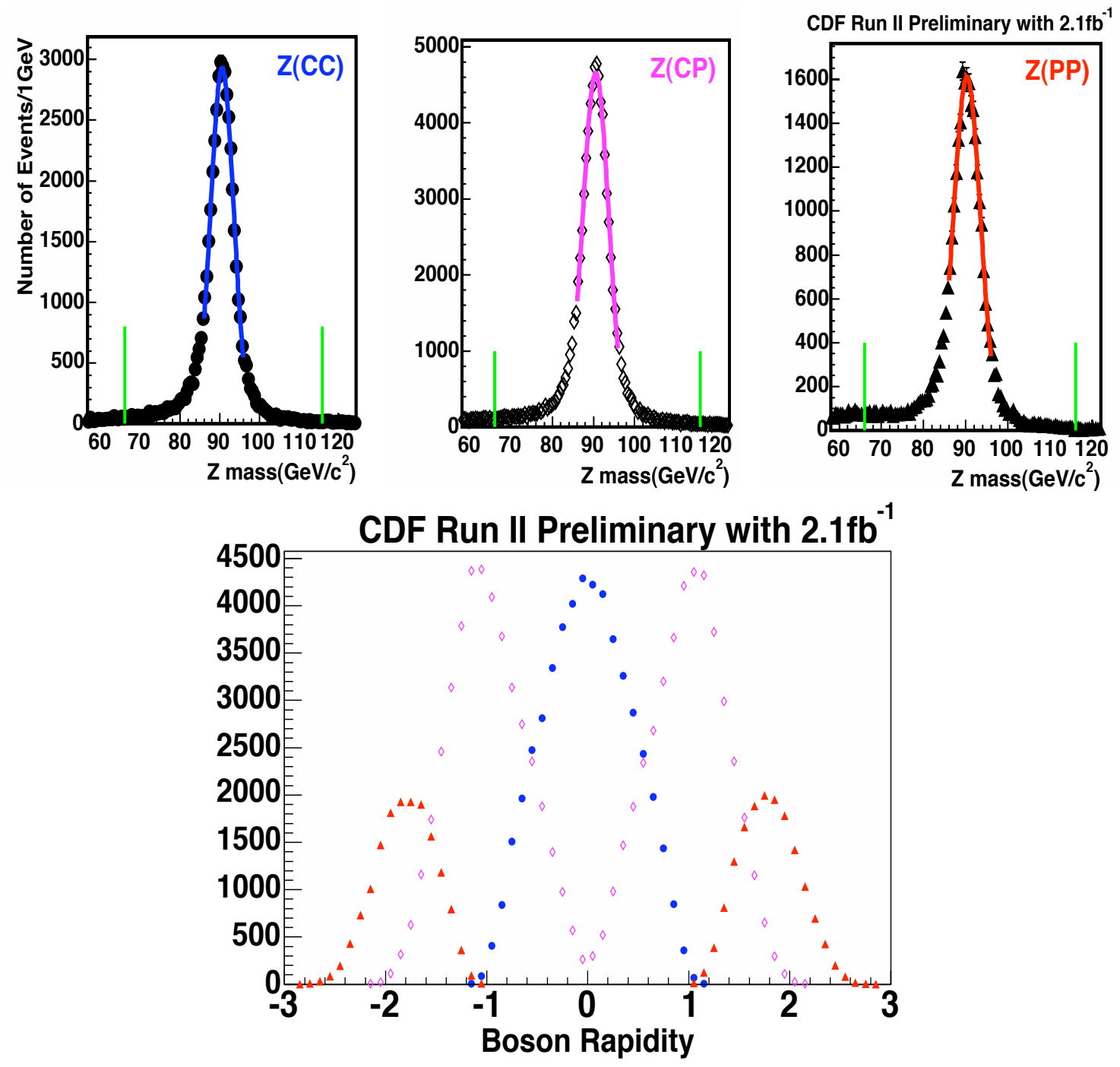

Figure 3.1: $Z / \gamma^{*} \mathrm{Z}$ Boson Mass and Rapidity Distribution. The plots on the top show the invariant mass distributions. The plots on the bottom shows the rapidity distribution. Here dots (blue), diamonds (magenta), and triangles (red) correspond to $\mathrm{Z}(\mathrm{CC}), \mathrm{Z}(\mathrm{CP})$, and $\mathrm{Z}(\mathrm{PP})$ events, respectively. 


\subsection{Energy Correction}

\subsubsection{PPR Energy}

Material in front of the calorimeter in the forward region affects the electron energy distribution. A comparison of the ratio of the PPR energy divided by the PEM $3 \times 3$ energy between data and $\mathrm{MC}$ is used to estimate the amount of material in front of the plug calorimeter. In the MC simulation, copper disks the front of the plug detector and phantom layers parallel to the beam line are used to tune the PPR response of the simulation to the data. There are ten phantom layers, for which four phantom layers are $0.9 \mathrm{~cm}$ thick, two are $0.4 \mathrm{~cm}$ thick, two $0.15 \mathrm{~cm}$ thick, one is $0.2 \mathrm{~cm}$ thick, and the another is $0.1 \mathrm{~cm}$ thick. The MC simulated with these materials is in a good agreement with the data for the ratio of the PPR energy to the total energy. Figure 3.3 shows this ratio (which is at the level of $2 \%$ ) versus $\eta_{\text {Det }}$. The PPR energy is added to the plug electron energy in the reconstruction of the $\mathrm{Z}$ Boson invariant mass.

\subsubsection{Plug Electron Energy Calibration in Data}

In the $\mathrm{Z}(\mathrm{PP})$ event topology sample, it is found that the $\mathrm{Z}$ mass for $y_{Z}>0$ is lower than the $\mathrm{Z}$ mass for $y_{Z}<0$ by by $0.9 \%$. (the average $\mathrm{Z}$ mass in the west region is $90.29 \pm 0.04$, and the mass $\mathrm{Z}$ mass in the east region is $89.50 \pm 0.05$.) In order to correct for this $y_{Z}>0$ versus d $y_{Z}<0$ discrepancy, an energy scale correction is applied to plug electrons for $\eta_{\text {Det }}>0$. The energy scale for the plug electron in $\eta_{\text {Det }}>0$ is $1.008 \pm 0.001$. This energy scale correction is also applied for electrons in the plug for both the $\mathrm{Z}(\mathrm{PP})$ and $\mathrm{Z}(\mathrm{CP})$ topology samples. 


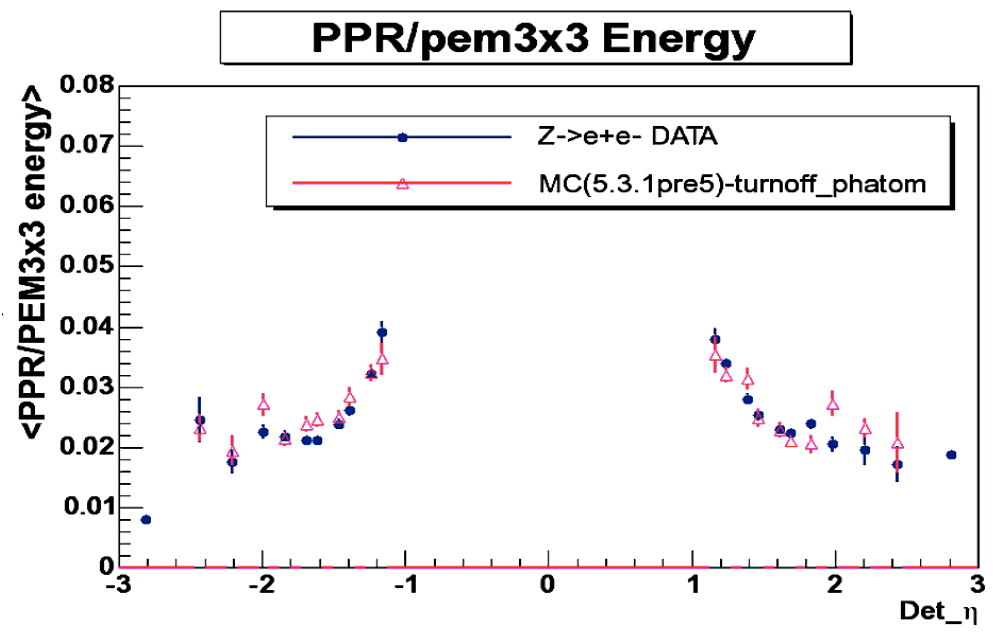

Figure 3.2: Ratio of PPR energy to total energy versus $\eta_{\text {Det }}$. The blue points are the data and the pink points are from the MC.

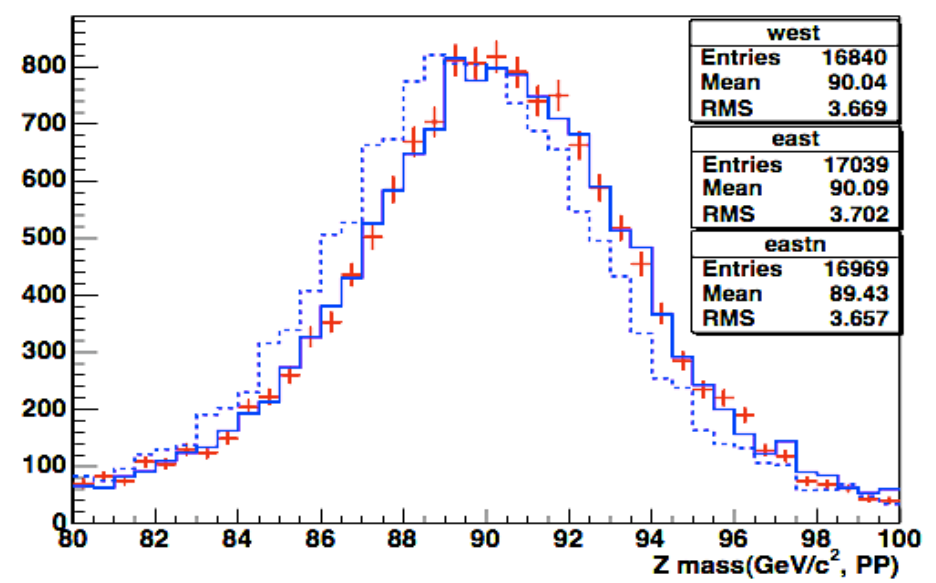

Figure 3.3: $\mathrm{Z}(\mathrm{PP})$ mass distribution for $y_{Z}>0$ and $y_{Z}<0$ in data. The red crosses are the $\mathrm{Z}$ mass distribution for $y_{Z}<0$ events, and the blue dashed line is the $\mathrm{Z}$ mass distribution for $y_{Z}>0$ events. The blue solid line is the $\mathrm{Z}$ mass distribution in $y_{Z}>0$ corrected by the energy scale correction for plug electrons. 


\subsubsection{Energy Scale in MC}

In fine tuning the MC samples, an energy scale correction is also applied to MC events to make the $\mathrm{Z}$ peak location the same for data and MC. This correction is determined by comparing the $\mathrm{Z}$ mass and $E_{T}$ distribution for data and $\mathrm{MC}$ for central and plug electrons in each event topology. We extract the overall energy scale $\left(S_{k}\right)$, as well as an additional constant $\left(S_{c}\right)$ term to be added to the energy resolution to account for additional resolution smearing that is seen in the data.

\section{Central Electrons in the $\mathrm{Z}(\mathrm{CC})$ sample}

The overall energy scale $\left(S_{k}\right)$ is extracted by minimizing $\chi^{2}$ in the comparison of the mass distribution of data and MC samples. Events in mass range, $70<M_{e e}<$ $100 \mathrm{GeV} / \mathrm{c}^{2}$ are used and $S_{k}$ is determined to be $S_{k}=0.9902 \pm 0.0002$. In Monte Carlo events, the generated electron energy is corrected by $S_{k}$, and then smeared with a Gaussian distribution. The smallest $\chi^{2}$ is obtained with $S_{c}=0.0094 \pm 0.0010$.

Even with the inclusion of the above $S_{k}$ and $S_{c}$ corrections, we find that the $E_{T}$ distributions in $\eta_{\text {Det }}$ (corresponding to the detector tower) show small disagreement between data and MC. The energy scale is a very important kinematic parameter for the determination of both $\mathrm{Z}$ boson mass and rapidity. Therefore, an extra energy scale correction versus $\eta_{\text {Det }}$ is measured and applied to the MC sample. The extra energy scale in each $\eta_{\text {Det }}$ range is determined by minimizing the $\chi^{2}$ between data and $\mathrm{MC} E_{T}$ distribution.

Figure 3.4 shows the extra energy scale for central electrons in the $\mathrm{Z}(\mathrm{CC})$ topology sample. After the application of an additional global scale factor (0.9997 \pm 0.0002$)$, the $\mathrm{Z}$ mass comparison for each $\eta_{\text {Det }}$ shows very good agreement between data and 


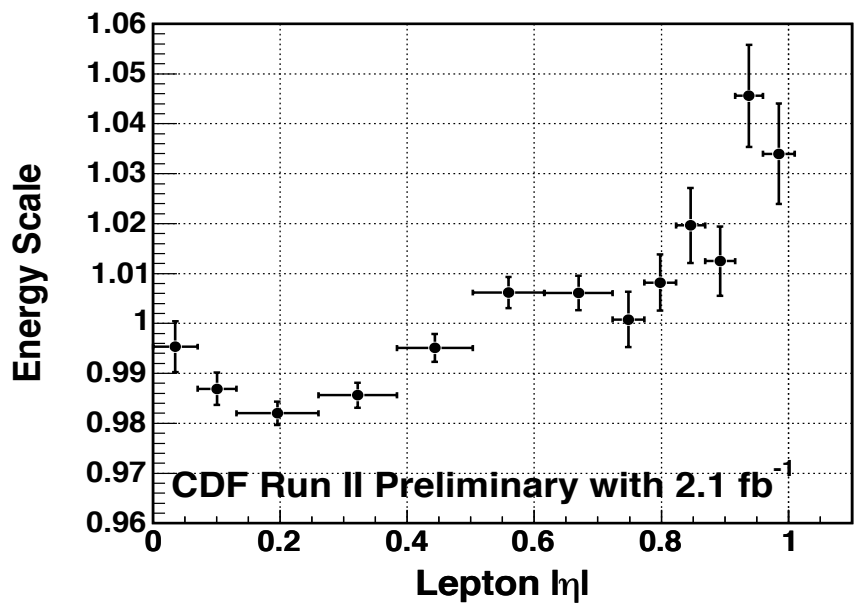

Figure 3.4: Extra energy scale in $\mathrm{Z}(\mathrm{CC})$. The plot shows the extra energy scale as a function of $\left|\eta_{D e t}\right|$.

MC. Figure 3.5 and 3.6 shows the Et and Z mass distribution in data and MC with different eta bins.

\section{Central Electrons in the $\mathrm{Z}(\mathrm{CP})$ sample}

The Monte Carlo energy sale, $S_{k}$ and the additional resolution smearing parameter $S_{c}$ measured with $\mathrm{Z}(\mathrm{CC})$ events are also used for the central electrons in the $\mathrm{Z}(\mathrm{CP})$ sample. The extra energy scale correction as a function of $\eta_{D e t}$ is measured in $\mathrm{Z}(\mathrm{CP})$ after applying $S_{k}$ and $S_{c}$. The extra energy scale is measured separately for events in which both electrons decay in same side and opposite side z direction, respectively. The $E_{T}$ distributions in each side shows different features. The $E_{T}$ distribution for opposite side events is lower than the $E_{T}$ for same side events. The energy scale estimated from each event category shows only a difference in level. Therefore, the energy scales in each category are combined and the combined energy scales are applied in the MC sample. The results are shown in Figure 3.7. Figure 3.8 and 3.9 

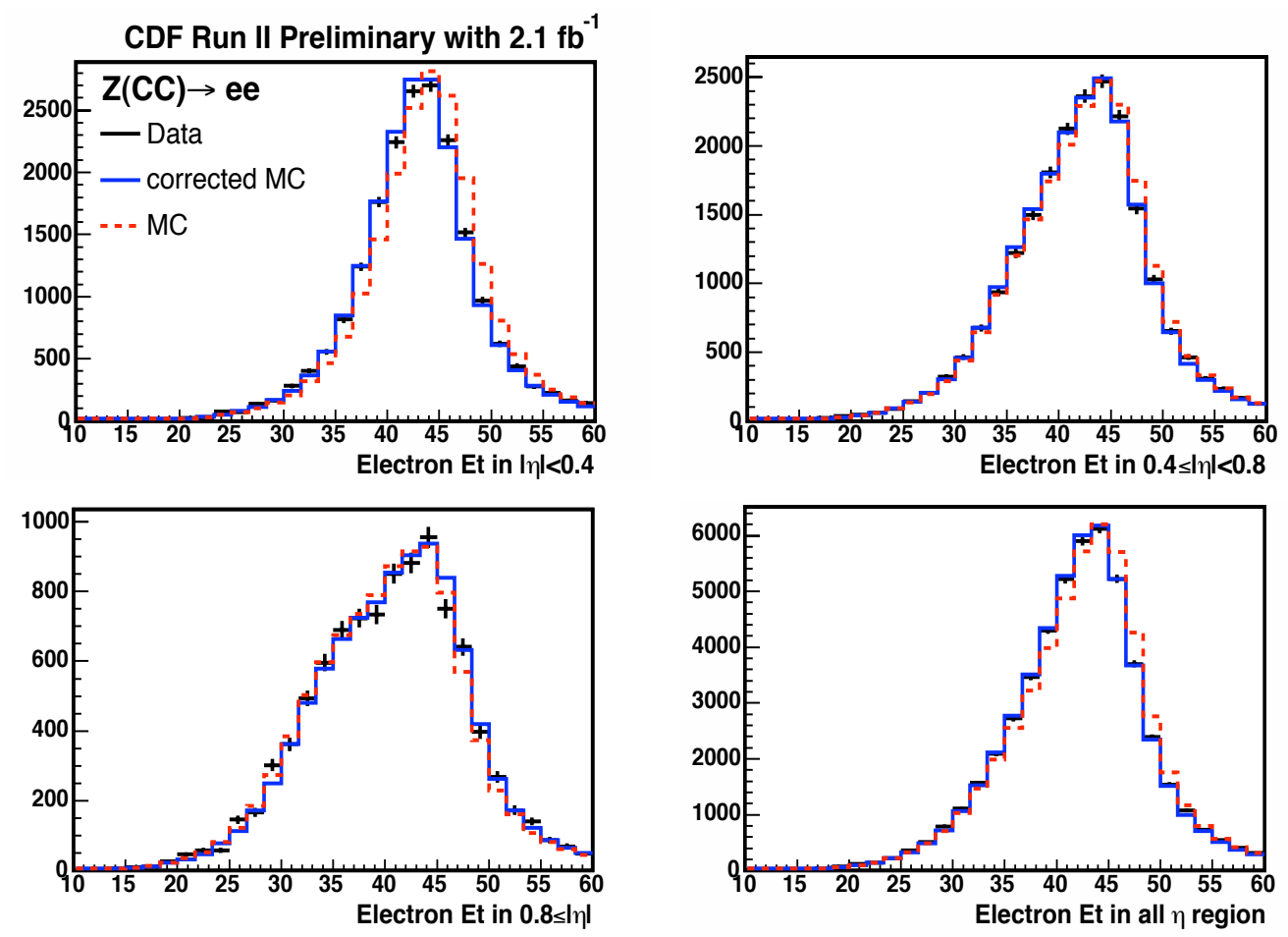

Figure 3.5: $E_{T}(\mathrm{GeV})$ distribution for $\mathrm{Z}(\mathrm{CC})$ events. All corresponding energy corrections are applied. The cross symbols are the data. The blue histogram is MC after applying the energy scale correction and the red dashed histogram is MC before the correction. 

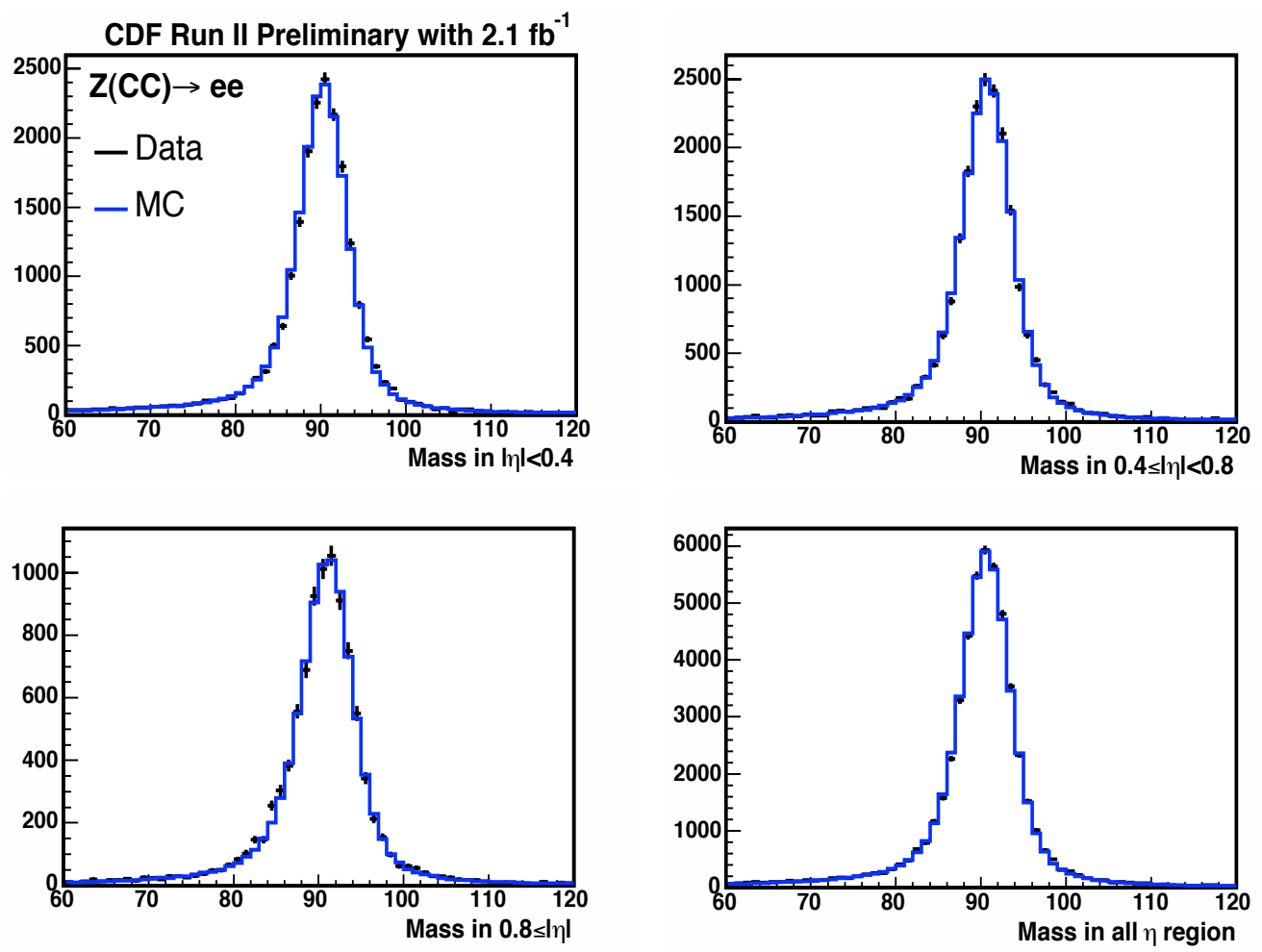

Figure 3.6: $\mathrm{Z}$ mass distribution for $\mathrm{Z}(\mathrm{CC})$ events. The cross symbols are data and the histogram is from MC. The solid blue line is the mass distribution after applying the energy scale correction in $\mathrm{MC}$. 
shows the Et and $\mathrm{Z}$ mass distribution for data and $\mathrm{MC}$ in different $\eta_{\text {Det }}$ regions.

\section{Plug Electrons in the Z(CP) Sample}

The energy scale of plug electrons in MC is first determined by comparing data and MC for the $E_{T}$ distribution of plug electrons in $\mathrm{Z}(\mathrm{CP})$ events as a function of $\eta_{\text {Det }}$. The overall energy scale correction for plug electrons and additional resolution smearing for each $\eta_{D e t}$ is estimated by comparing the $\mathrm{Z}$ mass distribution between data and MC. After all corrections, a comparison of the $\mathrm{Z}(\mathrm{CP})$ mass distribution between data and MC yields a final global energy scale factor of $0.9975 \pm 0.0002$. Figure 3.10 shows the energy scale and the resolution smearing factor for plug electrons in the $Z_{C P}$ sample. Figure 3.11 and 3.12 shows the $E_{T}$ and $\mathrm{Z}$ mass distributions in data versus $\mathrm{MC}$ as a function of $\eta_{\text {Det }}$.

The MC Z mass distribution of $\mathrm{Z}(\mathrm{CP})$ in $y \geq 0$ and $y<0$ does not match up exactly with the data after the MC energy scale is tuned as a function of $\left|\eta_{\text {Det }}\right|$ of the electron. Global factors are applied to MC in $y \geq 0$ and $y<0$ to correct it. The global factor is $1.0030 \pm 0.0005$ for $y \geq 0$ and $0.9966 \pm 0.0005$ for $y<0$. No further $\eta_{\text {Det }}$ dependencies are observed.

\section{Plug Electrons in the $\mathrm{Z}(\mathrm{PP})$ sample}

The energy scale for plug electrons in the $\mathrm{Z}(\mathrm{PP})$ sample is measured by the same method which is used to determined the energy scale of plug electrons in the $\mathrm{Z}(\mathrm{CP})$ sample. The energy scale of plug electrons is estimated using only same side events because the opposite side events are rejected in the $\mathrm{Z}(\mathrm{PP})$ selection criteria. After applying the energy scale from the $E_{T}$ distribution to $\mathrm{MC}$ events, the smearing factor and the global scale factor are measured by comparing the $\mathrm{Z}$ mass distribution for 

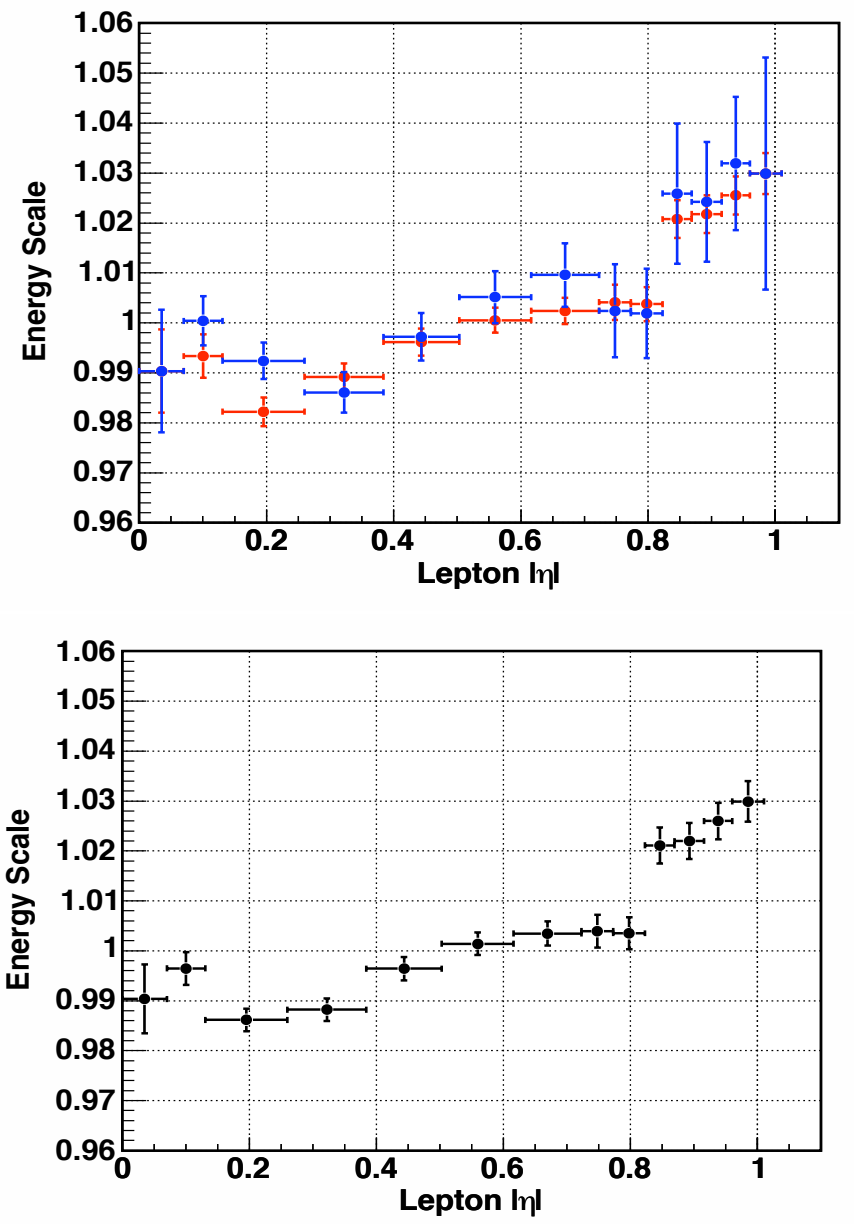

Figure 3.7: Central electron energy scale for $\mathrm{Z}(\mathrm{CP})$ events. The top plot shows the extra energy scale for the central electrons from same side events (triangle and blue points) and from opposite side events (square and red points). The same side (opposite side) events have both electrons decaying in same (opposite) side z direction. The bottom plot is the combined extra energy scale for the central electron in $Z_{C P}$ events as a function of $\left|\eta_{D e t}\right|$. 

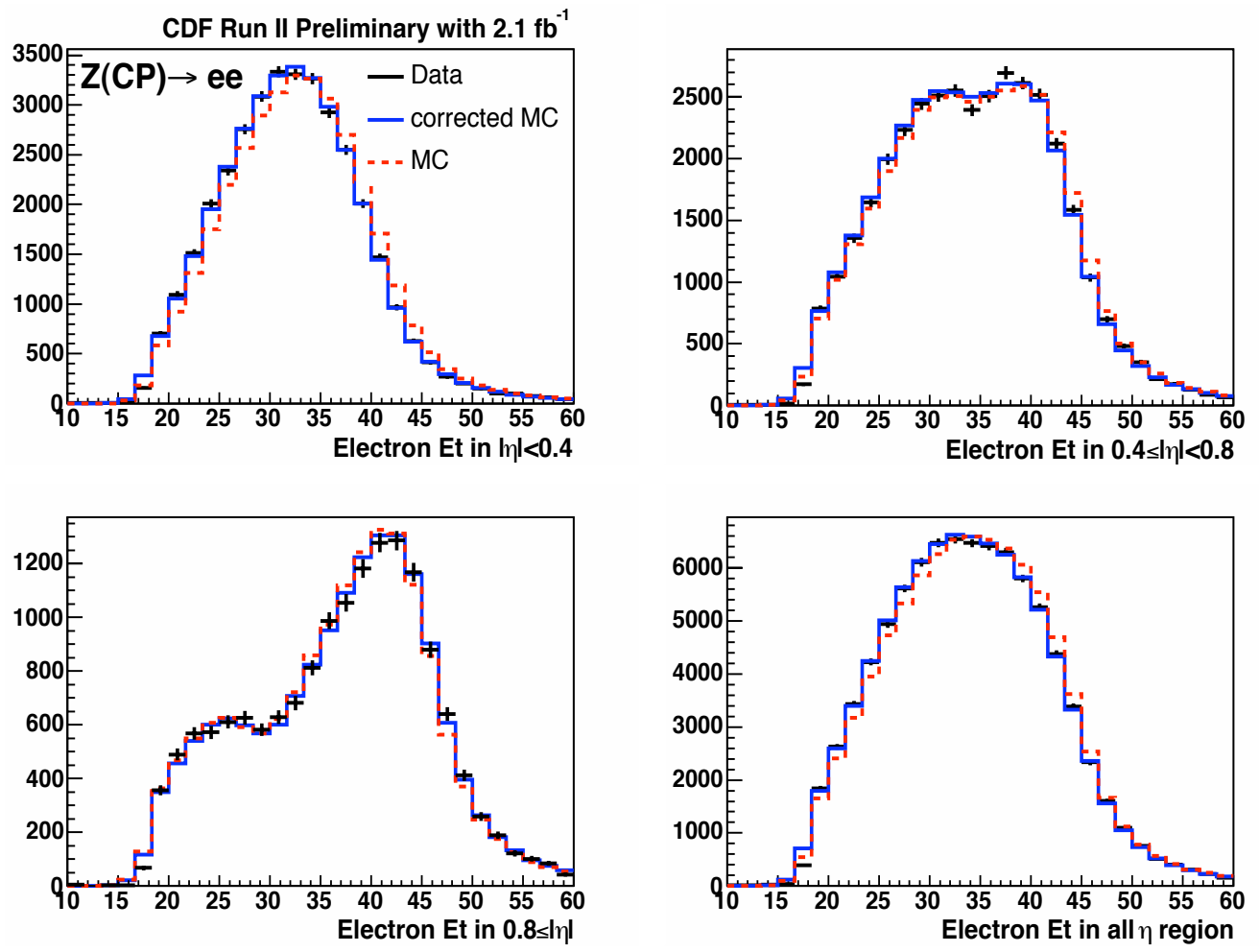

Figure 3.8: $E_{T}(\mathrm{GeV})$ distribution of central electrons in $\mathrm{Z}(\mathrm{CP})$ events. All energy corrections are applied to the MC sample. The cross symbols are the data. The blue histogram is MC after applying the energy scale correction and the red dashed histogram is $\mathrm{MC}$ before the energy correction. 

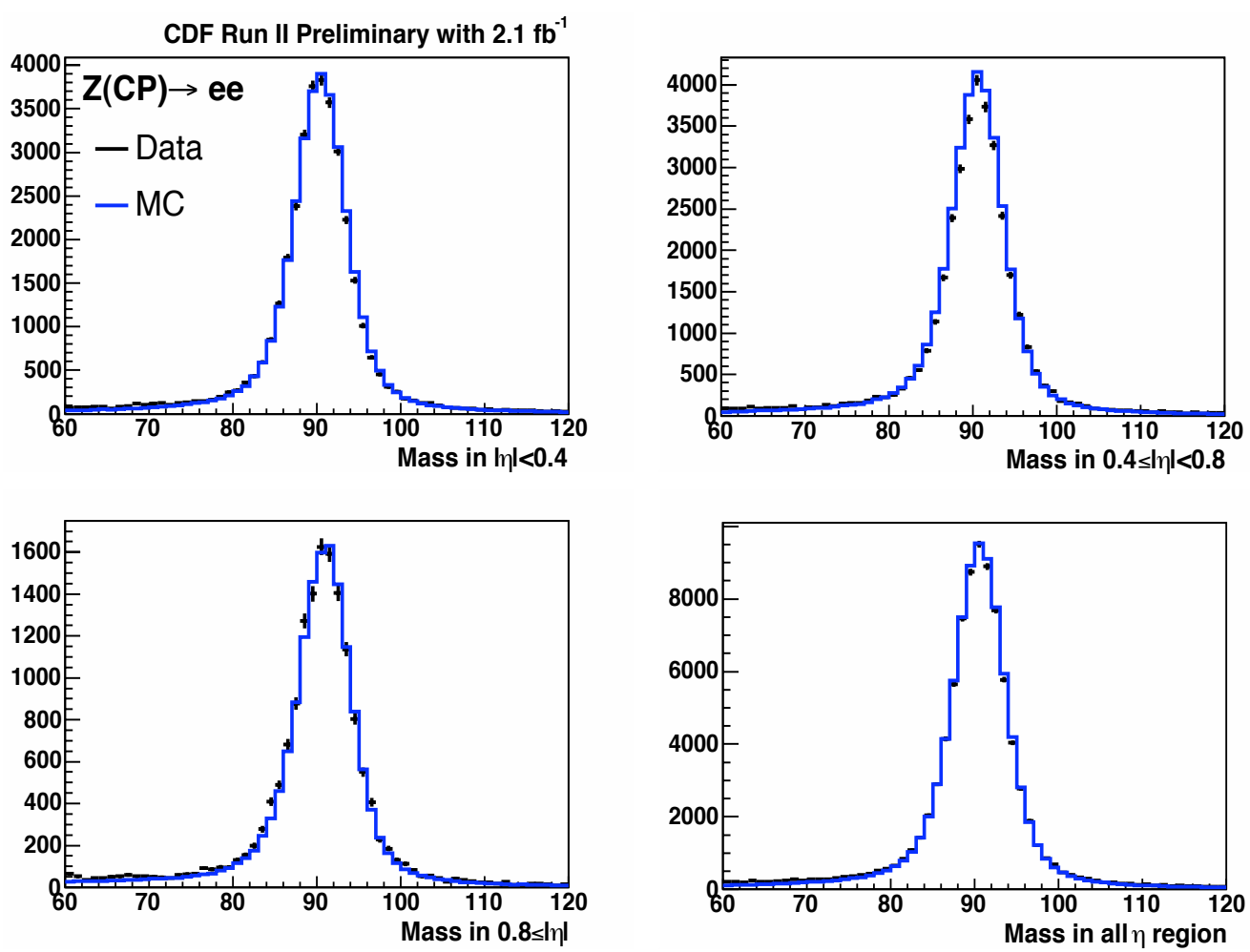

Figure 3.9: Z mass distribution in $\mathrm{Z}(\mathrm{CP})$ events. The cross symbols are the data and the histograms are MC. The solid blue line is the mass distribution after applying the energy scale correction in $\mathrm{MC}$. 

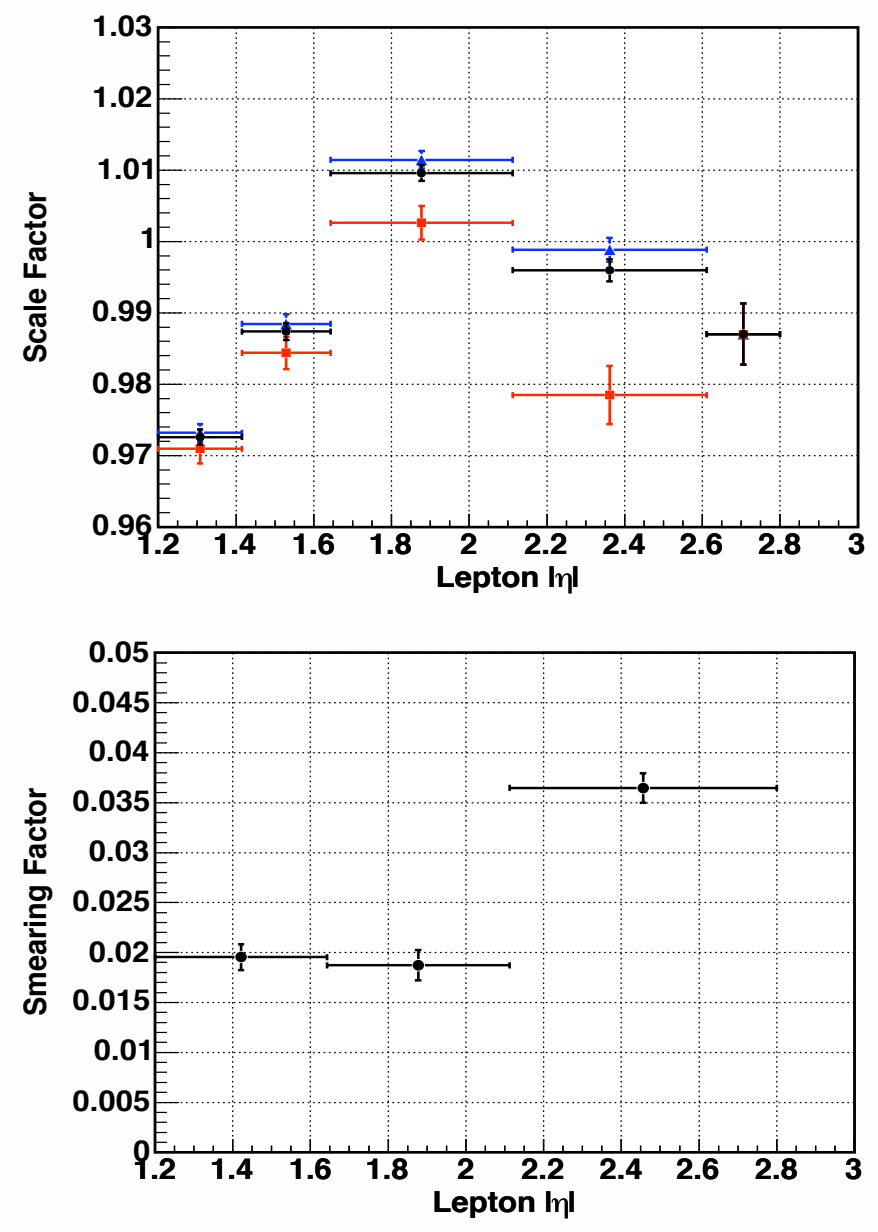

Figure 3.10: Plug electron energy scale for $\mathrm{Z}(\mathrm{CP})$ events. The top plot shows the energy scale for plug electrons in the $Z_{C P}$ sample as a function of $\left|\eta_{\text {Det }}\right|$. The triangle and blue dots are the energy scales from same side events and the square and red dots are the energy scale from opposite side events. The circle and black dots are the combined result. The bottom plot shows the resolution smearing factor for plug electrons. 

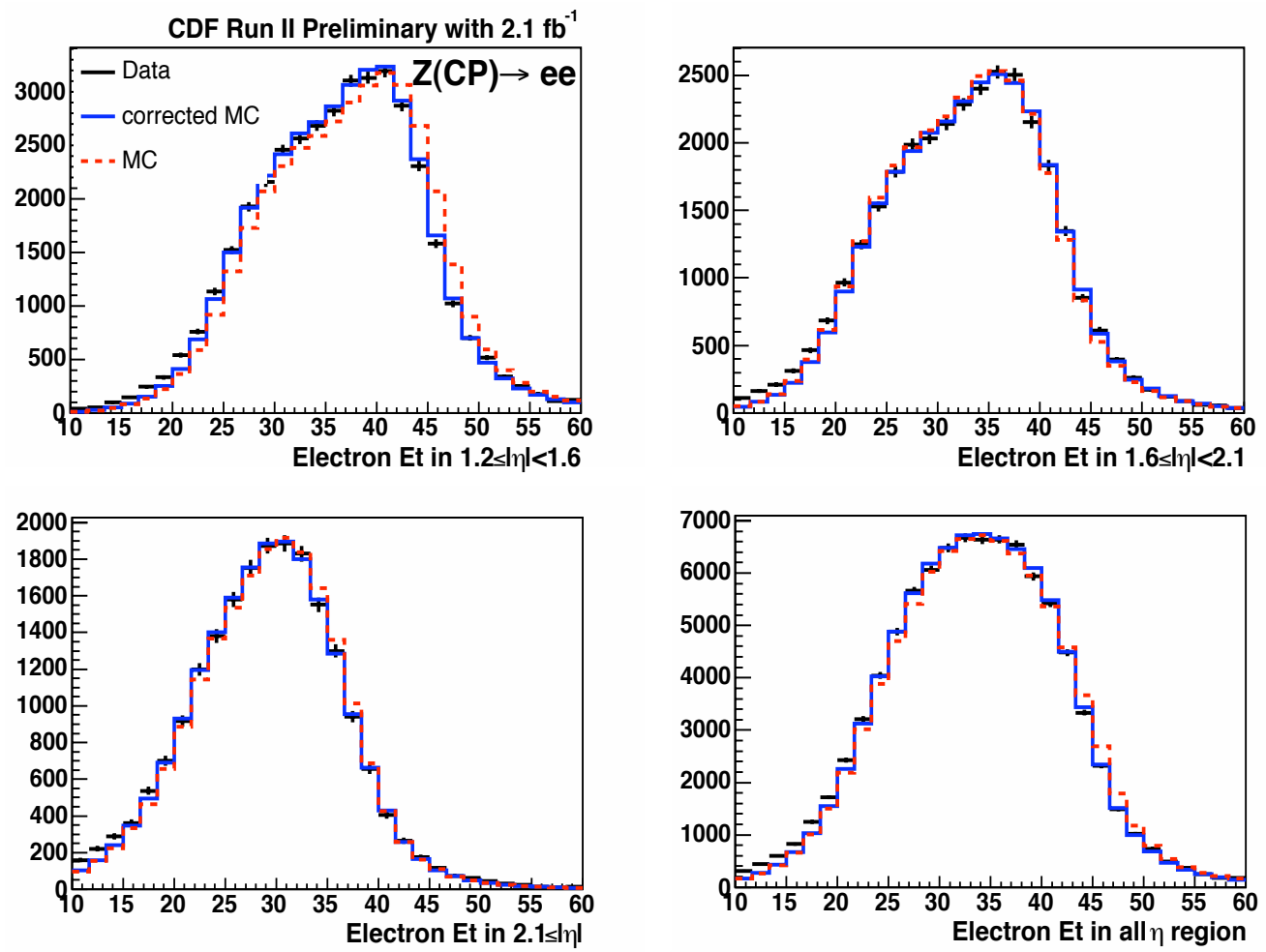

Figure 3.11: $E_{T}(\mathrm{GeV})$ distribution of plug electrons for $\mathrm{Z}(\mathrm{CP})$ events. All energy corrections are applied in MC. The cross symbols are data. The blue histogram is $\mathrm{MC}$ after applying the energy scale correction and the red dashed histogram is MC before the energy correction. 

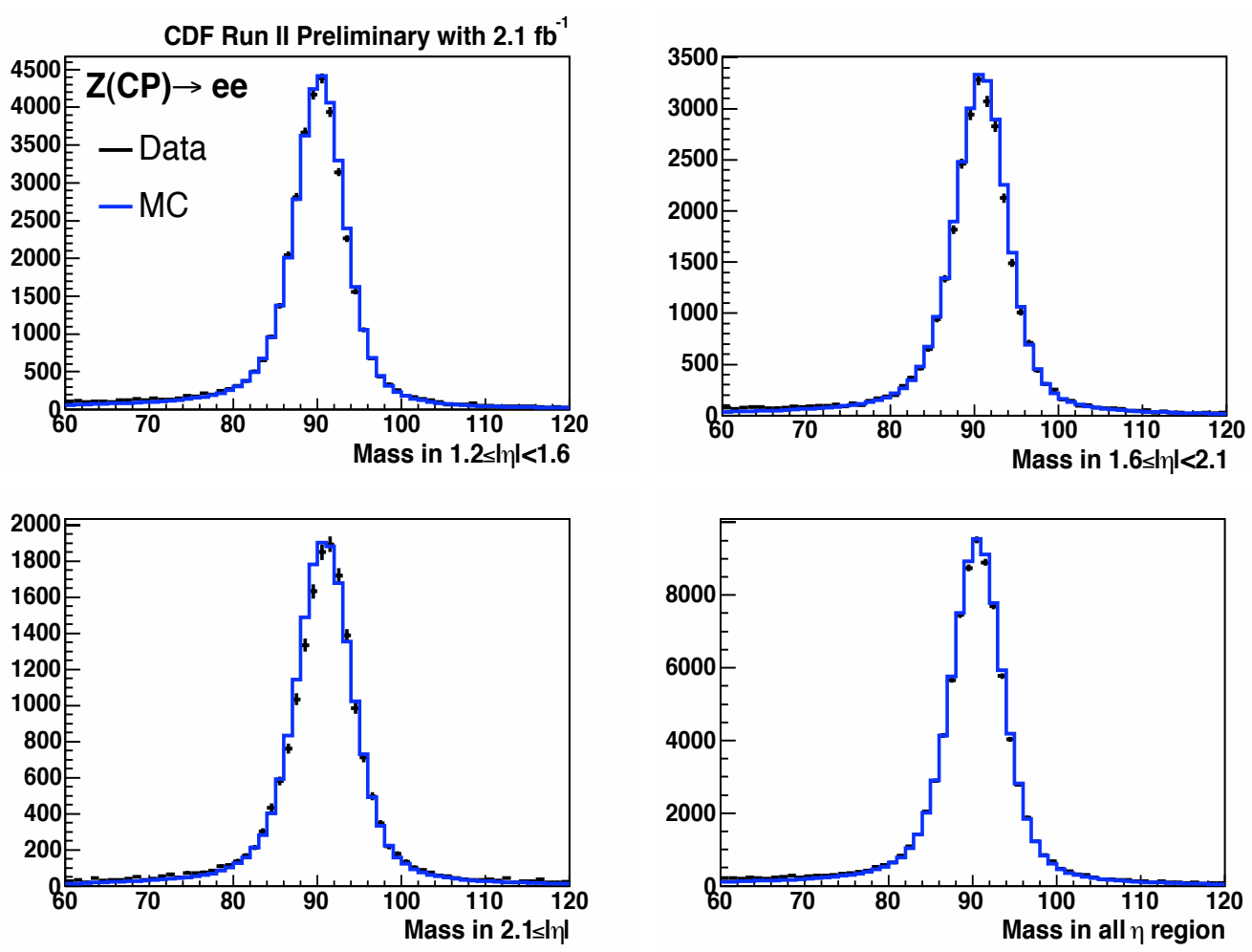

Figure 3.12: $\mathrm{Z}$ mass distribution for $\mathrm{Z}(\mathrm{CP})$ events. The plots are the mass distribution in data and $\mathrm{MC}$ after applying the plug energy scale for $Z_{C P}$ events. The cross symbols are data and the histograms are MC. The solid blue line is the mass distribution after applying the plug energy scale correction to the MC. The corrections for the central electron are applied. 


\begin{tabular}{|c|c|c|}
\hline & mass in data & mass in MC \\
\hline$Z(C C)$ & $90.60 \pm 0.02$ & $90.60 \pm 0.02$ \\
$Z(C P)$ & $90.53 \pm 0.02$ & $90.56 \pm 0.02$ \\
$Z(P P)$ & $90.26 \pm 0.03$ & $90.30 \pm 0.03$ \\
\hline & width in data & width in MC \\
\hline$Z(C C)$ & $2.92 \pm 0.02$ & $2.98 \pm 0.02$ \\
$Z(C P)$ & $3.12 \pm 0.02$ & $3.12 \pm 0.02$ \\
$Z(P P)$ & $3.22 \pm 0.04$ & $3.29 \pm 0.04$ \\
\hline
\end{tabular}

Table 3.3: Mean and width of the $\mathrm{Z}$ mass distribution. All energy corrections are applied to the MC sample.

data and MC. The global scale factor is measured to be $1.0044 \pm 0.0003$. Figure 3.13 shows the energy scale and the smearing factor for plug electrons in Z(PP) sample. Figure 3.14 and 3.15 compares the $E_{T}$ and $\mathrm{Z}$ mass distributions for data and $\mathrm{MC}$ as a function of $\eta_{\text {Det }}$.

All $\eta$ dependent energy corrections are applied to MC sample events using $\eta$ binby-bin corrections. Table 3.3 summarizes the mean and width of the mass distribution in data and MC from fitting the $\mathrm{Z}$ mass with Gaussian function in the $86<M_{e e}<$ $96 \mathrm{GeV} / \mathrm{c}^{2}$ region.

\section{Overall Scale Factor}

In the previous chapter, the energy scale for MC is estimated for each topology assuming that the background in the sample does not affect the determination of the energy scale. To confirm this assumption, the overall energy scale and smearing factor are measured with and without the background subtraction in data. The overall energy scale and smearing factor are estimated by comparing the $\mathrm{Z}$ mass distribution between data and MC. The dielectron mass distribution for the background 

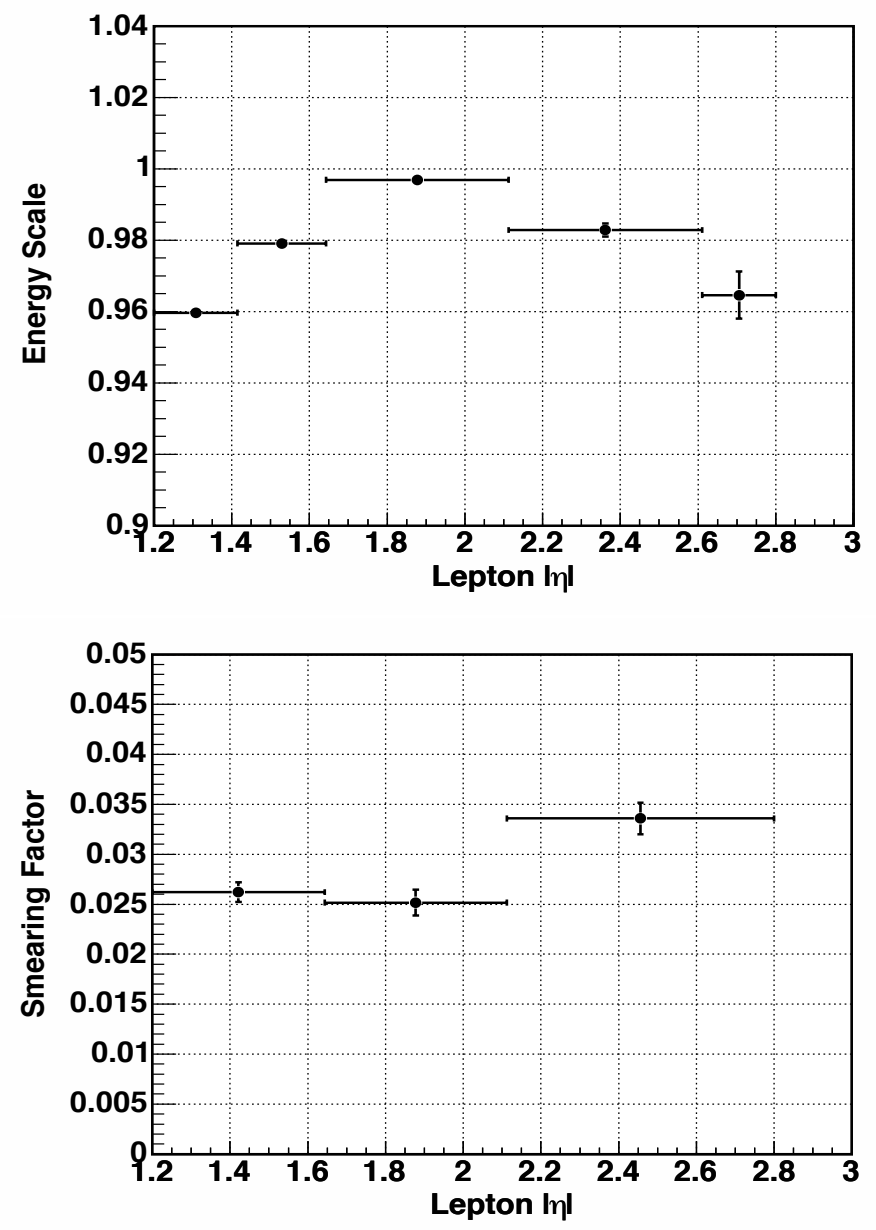

Figure 3.13: Plug electron energy scale for $\mathrm{Z}(\mathrm{PP})$ events. The top plot shows the energy scale for $Z_{P P}$ plug electrons as a function of $\left|\eta_{D e t}\right|$. The bottom plot shows the smearing factor for plug electrons. 

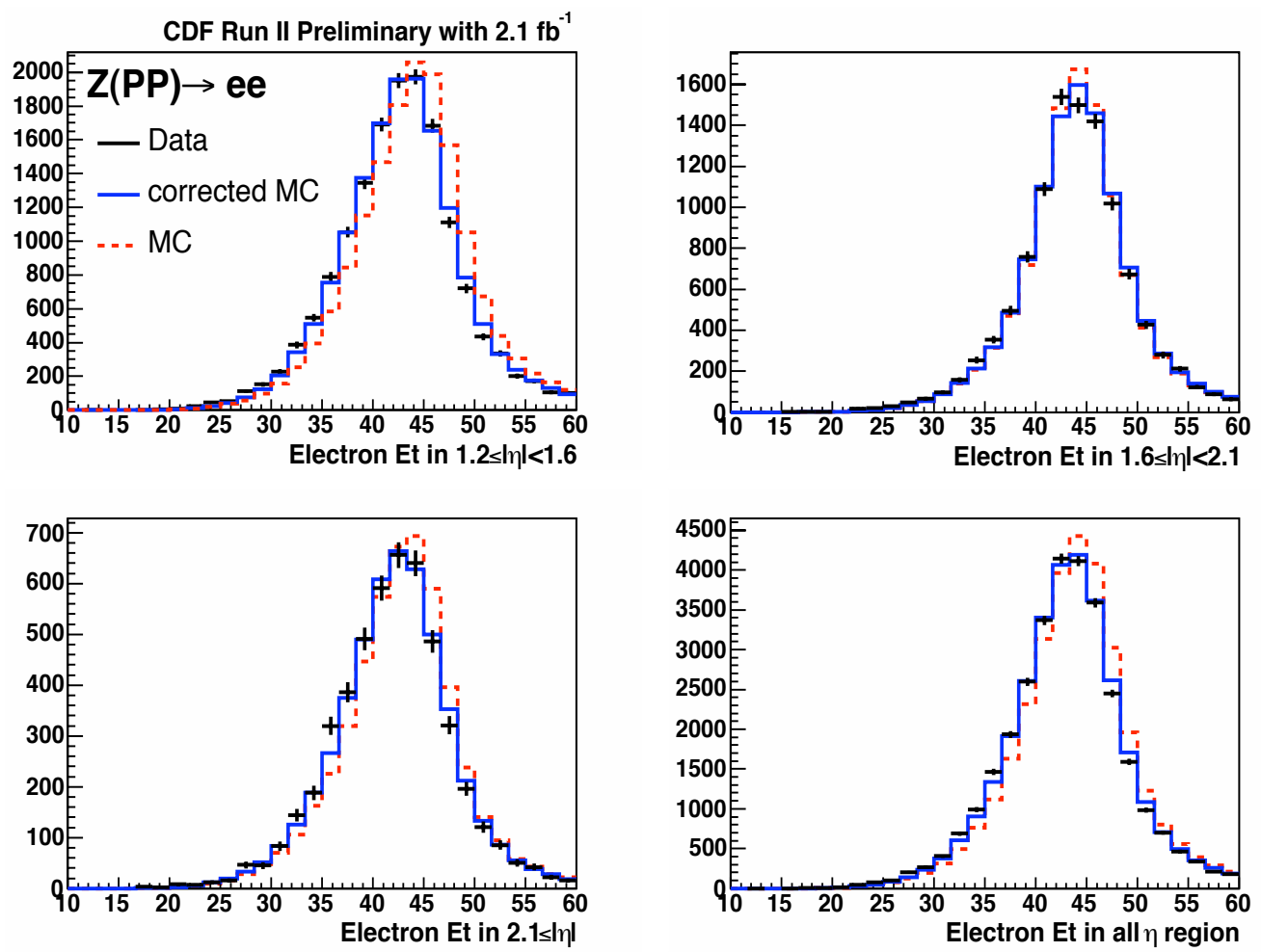

Figure 3.14: $E_{T}(\mathrm{GeV})$ distribution of plug electron for $\mathrm{Z}(\mathrm{PP})$ events. All corrections for plug electrons are applied in MC. The cross symbols are data and the blue histogram is MC after applying the energy correction. The red dashed histogram is MC before the energy correction. 

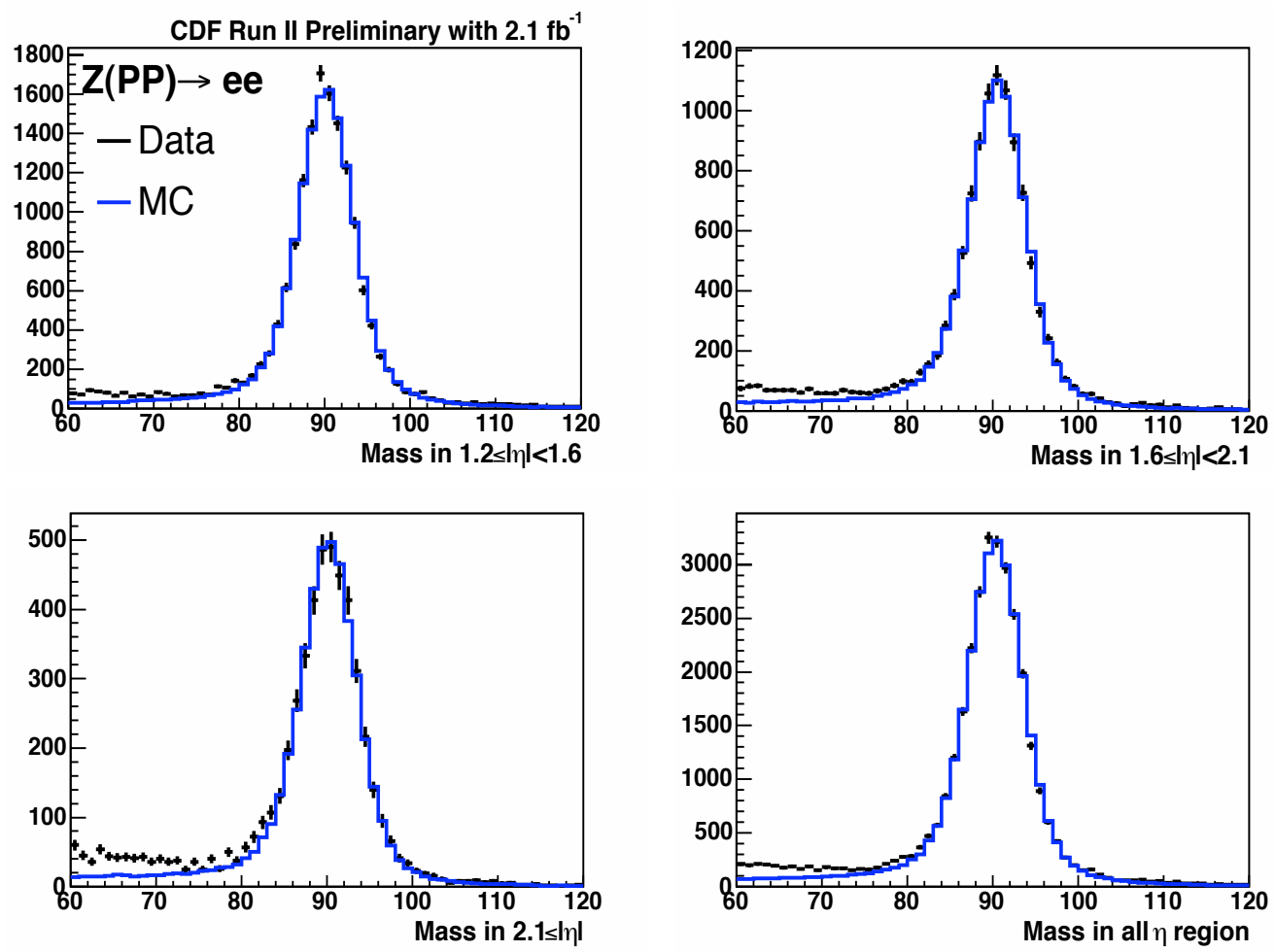

Figure 3.15: Z mass distribution for $\mathrm{Z}(\mathrm{PP})$ events. The cross symbols are data and the histograms are MC. The solid blue line is the mass distribution after applying the energy scale corrections in MC. 
events is obtained by applying the anti-electron selection to one of two electrons to select the jet-like object. This mass distribution is normalized to the measured background rate as described in section 4 . The normalized mass distribution is subtracted from the data as the background contribution. Table 4.1 in section 4 describes the selection criteria for the background sample. The energy scale of the central electron is estimated using the $\mathrm{Z}(\mathrm{CC})$ sample. The energy scale of the central electron with and without background subtraction is $0.9881 \pm 0.0002$ for both samples. The smearing factors for central electrons with and without background subtraction is $0.0121 \pm 0.0009$ and $0.0120 \pm 0.0009$, respectively. The measured energy scale and smearing factor for central electrons is applied to the central leg in $\mathrm{Z}(\mathrm{CP})$ events. The energy scale and smearing factor for plug electron is measured using the $\mathrm{Z}(\mathrm{CP})$ sample after the correction for the central electron energy is applied. The energy scale for plug electrons is also measured in $\mathrm{Z}(\mathrm{PP})$ sample with and without background subtraction. Table 3.4 compares the energy scales and smearing factors with and without background subtraction. The energy scales and smearing factors with and without background subtraction are consistent within a $1 \delta$ uncertainty except the smearing factor of $\mathrm{Z}(\mathrm{CC})$. However, the smearing factor in $\mathrm{Z}(\mathrm{CC})$ topology is not large and the difference between the smearing factor measured with and without the background subtraction is negligible in the mass distribution. Therefore, the background does not affect the determination of the electron energy scales.

\subsection{The ZVertex Correction in MC}

The variable ZVertex is the proton-antiproton interaction point along the $\mathrm{z}$ direction of the beam line with respect to the center of the detector, which is defined as 
(a) Background Subtraction

\begin{tabular}{|l|c|c|}
\hline Electron & Energy Scale & Smearing \\
\hline Central in $Z(C C)$ & $0.9881 \pm 0.0002$ & $0.0210 \pm 0.0009$ \\
Plug in $Z(C P)$ & $0.9889 \pm 0.0003$ & $0.0220 \pm 0.0009$ \\
Plug in $Z(P P)$ & $0.9851 \pm 0.0003$ & $0.0279 \pm 0.0007$ \\
\hline
\end{tabular}

(b) No Background Subtraction

\begin{tabular}{|l|c|c|}
\hline Electron & Energy Scale & Smearing \\
\hline Central in $Z(C C)$ & $0.9881 \pm 0.0002$ & $0.0120 \pm 0.0009$ \\
Plug in $Z(C P)$ & $0.9889 \pm 0.0003$ & $0.0214 \pm 0.0010$ \\
Plug in $Z(P P)$ & $0.9851 \pm 0.0003$ & $0.0272 \pm 0.0007$ \\
\hline
\end{tabular}

Table 3.4: Mean and width of the Z mass distribution. All energy corrections are applied to the MC sample.

ZVertex $=0$. The ZVertex location is used to calculate $E_{T}$ (the energy in the transverse direction), and therefore affects the calculated $\mathrm{Z}$ boson mass and rapidity. The ZVertex distribution in the MC sample is compared with data to ensure that the MC properly simulates the ZVertex distribution. After all Z selections cuts are applied, the ZVertex distributions for all event topologies (CC, CP, and PP) are combined in data and compared to the MC simulation. The MC sample that is used has been tuned for all energy calibration corrections and efficiencies.

Figure 3.16 shows the ratio of the ZVertex distribution for data and MC events. The plot indicates that in the MC simulation there are more events than in the data in the regions of of large positive and large negative ZVertex (i.e away from the center of the detector). The large ZVertex region is far from the center of the detector, where the tracking efficiency is lower than in the central region. Therefore, this region may be more sensitive to the tracks from the underlying event. In addition, possible background contaminations may also affect the ZVertex distribution.

To determine whether the difference between data and Monte Carlo originates 
from possible background events, we select only 2 track events in $\mathrm{CC}, \mathrm{CP}$, and PP topology and compare the ratio of the ZVertex distributions (data/MC) for all Z events versus for only 2 track events. The background level in 2 track events is negligible, so possible backgrounds cannot affect the ZVertex distribution for this sample. Figure 3.16 shows that the ratio of data/MC for the ZVertex distribution for 2 track events is consistent with the ratio for all $\mathrm{Z}$ events. Therefore, the ZVertex difference between data and MC does not originate from background events. Since the ZVertex distribution affects the acceptance and efficiencies it should be modeled correctly in the MC. The ratio of data to MC for the Zvertex distribution is applied to MC events to correctly model the ZVertex distribution in MC using a bin by bin correction factor. 


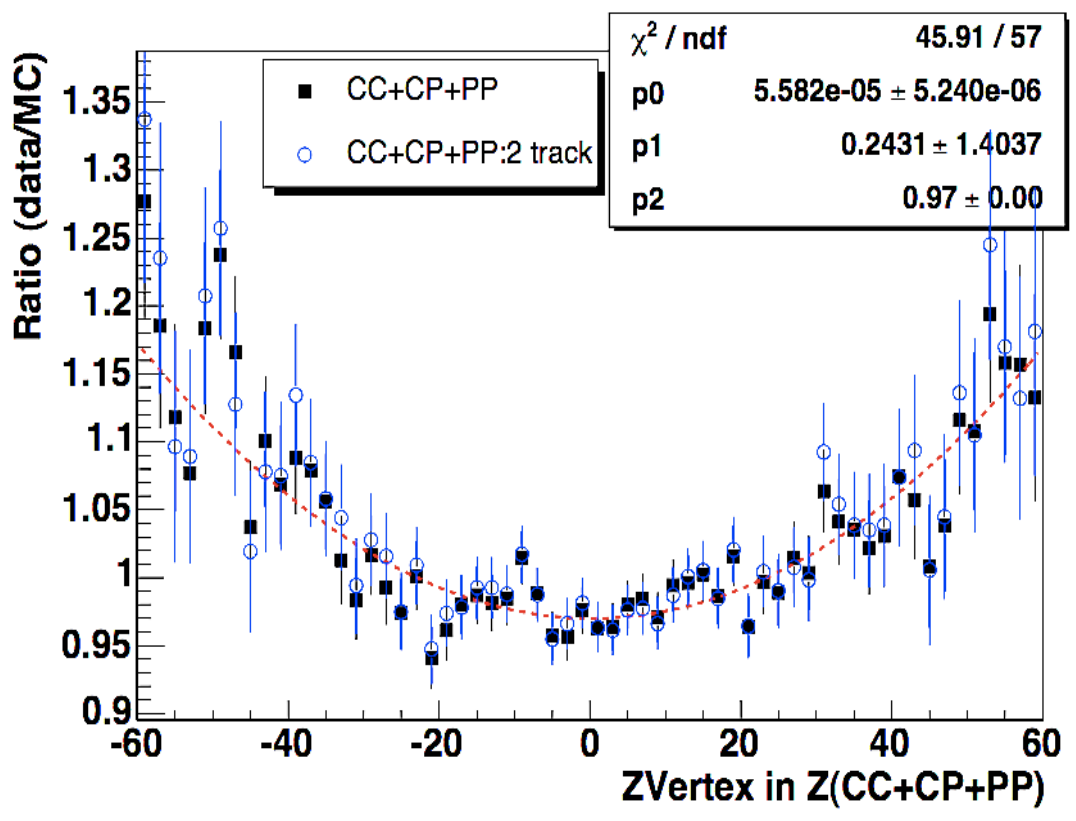

Figure 3.16: The ratio of the number of events in data to $\mathrm{MC}$ as a function of the ZVertex. Here, the ZVertex distribution for all $\mathrm{Z}$ events $(\mathrm{CC}+\mathrm{CP}+\mathrm{PP})$ is compared between data and MC. In order to remove the effect of all possible backgrounds only 2 track events for the $\mathrm{CC}, \mathrm{CP}$, and PP topologies are selected. The comparison of the ZVertex distributions between data and $\mathrm{MC}$ events is shown the plot as the empty dot symbols. (Note that the background level in 2 track events is negligible)

The ratio of data/MC versus Zvertex for all $\mathrm{Z}$ events is consistent with the ratio for 2 track events only. Therefore, any possible background effects is negligible. The ratio of the Zvertex distributions for data and MC using all $\mathrm{Z}$ events is fitted using the function $f(x)=p 0 \times(x-p 1)^{2}+p 2$. 


\subsection{Trigger Requirements and Efficiencies}

\subsubsection{Trigger Requirements}

The high $p_{T}$ electron trigger data set at CDF is used for the electroweak, exotic and top analyses, all of which include $\mathrm{W}$ and $\mathrm{Z}$ decays to electrons. The cuts on this sample are left intentionally loose in order to be able to extract efficiency corrections for various cuts using the same data set. Two subsets of the data set are used in this analysis. The first is the $Z_{-} N O T R A C K$ trigger path, which requires two EM objects with high $E_{T}\left(E_{T}>18 \mathrm{GeV}\right)$. The other is the ELECTRON_CENTRAL_18 trigger path, which requires at least one central electron with high $E_{T}\left(E_{T}>18 \mathrm{GeV}\right)$ and an associated track.

For the $\mathrm{Z}(\mathrm{CC})$ and $\mathrm{Z}(\mathrm{CP})$ topologies, events are accepted from either the ELECTR$O N_{-} C E N T R A L_{-} 18$ or the $Z_{-} N O T R A C K$ trigger paths. This results in increased statistics for the $\mathrm{Z}(\mathrm{CC})$ and $\mathrm{Z}(\mathrm{CP})$ sample. The ELECTRON_CENTRAL_18 trigger path does not include events in the plug region. Therefore, only the $Z_{-} N O T R A C K$ trigger path is used for the $\mathrm{Z}(\mathrm{PP})$ topology. Table 3.5 summarizes the trigger condition at each level.

\subsubsection{Trigger Efficiencies}

The trigger efficiency corresponding to the EM calorimeter triggers is mostly affected by the $E_{T}$ distribution of the electron. The trigger efficiency of the tracking requirement is affected by the geometry of the detector because the $\cos \theta$ effect on $d E / d x$ and central wire supports give $\eta_{\text {Det }}$ dependence. Therefore, the efficiency of the calorimeter component of the triggers is measured as a function of $E_{T}$, and the efficiency of the tracking component is measured as a function of $\eta$. 


\begin{tabular}{|c|l|}
\hline \multicolumn{2}{|c|}{$Z_{-}$NOTRACK } \\
\hline Trigger Path & Requirement \\
\hline L1_TWO_EM8 & Number of EmCluster $\geq 2$ \\
& $H a d / E m($ central $)<0.125$ \\
& $H a d / E m($ plug $)<0.0625$ \\
& $E_{T}>8 \mathrm{GeV}$ for both electrons \\
\hline L2_TWO_EM16 & Number of EmCluster $\geq 2$ \\
& $H a d / E m<0.125$ for both electrons \\
& $E_{t}>16 \mathrm{GeV}$ for both electrons \\
\hline L3_TWO_ELECTRON18 & Number of EmObject $\geq 2$ \\
& $E_{T}>18 \mathrm{GeV}$ for both electrons \\
\hline
\end{tabular}

\begin{tabular}{|c|l|}
\hline \multicolumn{2}{|c|}{ ELECTRON_CENTRAL_18 } \\
\hline Trigger Path & Requirement \\
\hline L1_CEM8_PT8 & Calorimeter Region $=$ Central \\
& Number of EmCluster $\geq 1$ \\
& $E_{T}($ central $)>8 \mathrm{GeV}$ \\
& Had/Em $($ central $)<0.125$ \\
& Number of XFT layers $\geq 4$ \\
& Track $p_{T}>8 \mathrm{GeV} / \mathrm{c}$ \\
\hline L2_CEM16_PT8 & Calorimeter Region $=$ Central \\
& Number of EmCluster $\geq 1$ \\
& $E_{T}>18 \mathrm{GeV}$ \\
& Had/Em $<0.125$ \\
& Number of XFT layers $\geq 4$ \\
& Track $p_{T}>8 \mathrm{GeV}$ \\
\hline L3_CENTRAL_ELECTRON18 & Calorimeter Region $=$ Central \\
& Number of EmObject $\geq 1$ \\
& $E_{T}>18 G e V$ \\
& Had/Em $<0.125$ \\
& Track $p_{T}>8 \mathrm{GeV}$ \\
& Number of ZVertex $\geq 2$ \\
& $L_{\text {shr }}<0.4$ \\
& $\Delta z<8$ \\
\hline
\end{tabular}

Table 3.5: Trigger path requirements. 


\section{Z_NOTRACK Trigger}

The efficiency of the $Z_{-} N O T R A C K$ trigger is measured for central and plug electrons. The $\mathrm{Z}(\mathrm{CP})$ event sample is a good sample for the determination of the trigger efficiency for both central and plug electrons. The $\mathrm{Z}(\mathrm{CP})$ sample is a high statistics and low background sample. In addition, electrons from central and plug legs in the $\mathrm{Z}(\mathrm{CP})$ sample are independent of each other, which make it possible to measure the trigger efficiency for the central and plug electron separately by requiring different trigger paths.

The PLUG_ELECTRON_20 trigger path requires at least one plug electron with $E_{T}>20 \mathrm{GeV}$. This requirement yields an unbiased sample of central electrons triggered by the plug leg in $\mathrm{Z}(\mathrm{CP})$ events. The trigger efficiency of the plug electron is measured by requiring that the event has been selected by the ELECTRON_CENTR$A L \_18$ trigger. The ELECTRON_CENTRAL_18 trigger only affects central electrons, and provides an unbiased sample of plug electrons. All electron identification selections which are applied to clean the sample (except $E_{T}$ of the probed electron) are described in Table 3.1 and Table 3.2 . The mass window, $66<M<116 \mathrm{GeV} / \mathrm{c}^{2}$ is used for the determination of efficiency. The trigger efficiency for central and plug electrons $\left(\epsilon_{C}\right.$ and $\left.\epsilon_{P}\right)$ as a function of $E_{T}$ are defined as :

$$
\begin{aligned}
& \epsilon_{C}\left(Z_{-} N O T R A C K\right)=\frac{Z(C P) \text { passing } Z_{\_} N O T R A C K \& \text { PLUG_ELECTRON_20 }}{Z(C P) \text { passing PLUG_ELECTRON_20 }} \\
& \epsilon_{P}\left(Z_{-} N O T R A C K\right)=\frac{Z(C P) \text { passing } Z_{\_} N O T R A C K \& \text { ELECTRON_CENTRAL_18 }}{Z(C P) \text { passing ELECTRON_CENTRAL_18 }}
\end{aligned}
$$


The $Z_{-} N O T R A C K$ trigger efficiency of the plug electron is measured in each

$\eta$ range because the plug electron energy in the forward region is affected by the larger resolution smearing. Figure 3.17 shows the $Z_{-} N O T R A C K$ trigger efficiency for central and plug electrons as a function of $E_{T}$.

\section{ELECTRON_CENTRAL_18 Trigger}

The ELECTRON_CENTRAL_18 trigger efficiency has been measured by the Joint Physics Group at CDF.[13] They measure the efficiency in each trigger level, run range (time period), calorimeter and track trigger respectively. The efficiency for the calorimeter component is measured as a function of $E_{T}$, and the efficiency for the tracking component is measured as a function of $\eta$ of the electron. Figure 3.18 shows the efficiencies of ELECTRON_CENTRAL_18 trigger path measured using the data collected in one time period ( September, 2006 to November, 2006 ).

\section{Trigger Efficiency versus Boson Rapidity}

The trigger efficiencies of the $Z_{-} N O T R A C K$ and ELECTRON_CENTRAL_18 samples are convoluted into boson rapidity using the $Z \rightarrow$ ee $\mathrm{MC}$ sample. The trigger efficiency versus boson rapidity is calculated using event weighting in MC. The weighting factor for $\mathrm{Z}(\mathrm{CC})$ is defined as :

$$
w_{C C}\left(E_{T}^{1}, E_{T}^{2}, \eta^{1}\right)=1-\left(1-\epsilon_{e}\left(E_{T}^{1}, \eta^{1}\right)\right) \times\left(1-\epsilon_{z}\left(E_{T}^{1}\right) \times \epsilon_{z}\left(E_{T}^{2}\right)\right)
$$

where $\epsilon_{e}$ is the efficiency of the ELECTRON_CENTRAL_18 trigger and $\epsilon_{z}$ is the efficiency of the $Z_{-} N O T R A C K$ trigger. The $E_{T}^{1}$ and $E_{T}^{2}$ are the transverse energies of the electrons corrected by the energy scale, and $E_{T}^{1}$ is selected to be greater than $E_{T}^{2}$. The weighting factor for $\mathrm{Z}(\mathrm{CP})$ is :

$$
w_{C P}\left(E_{T}^{c}, E_{T}^{p}, \eta^{c}\right)=1-\left(1-\epsilon_{e}\left(E_{T}^{c}, \eta^{c}\right)\right) \times\left(1-\epsilon_{z}\left(E_{T}^{c}\right) \times \epsilon_{z}\left(E_{T}^{p}\right)\right)
$$



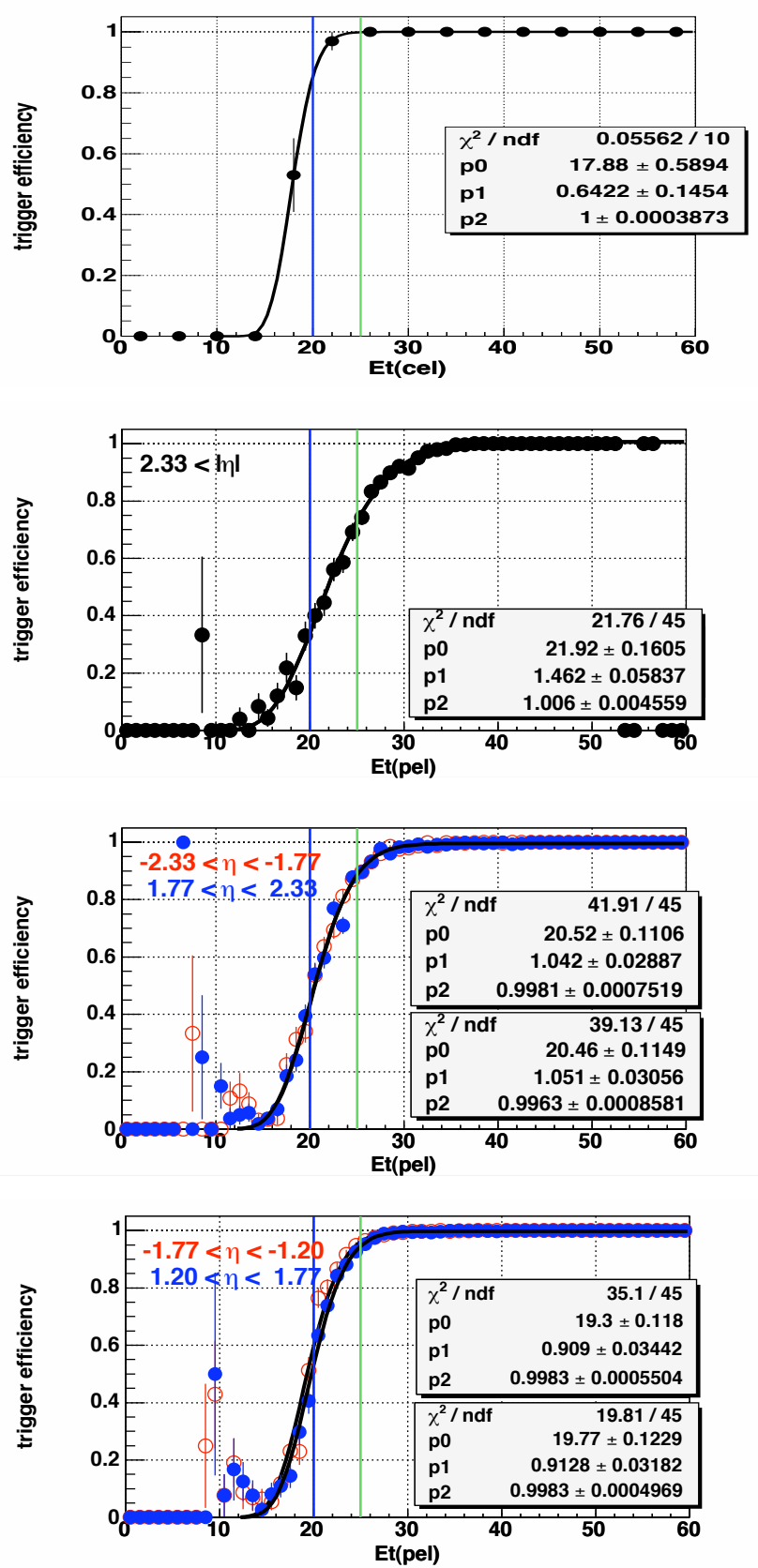

Figure 3.17: $Z_{-} N O T R A C K$ trigger efficiency in $E_{t}$. The first plot shows the trigger efficiency for central electrons, the other plots are the trigger efficiency versus $E_{T}$ for plug electrons in different $\eta$ ranges. In the high $\eta$ region, the events for $\eta>0$ and $\eta<0$ are combined to get more statistics. In the third and fourth plots, the open dots are the efficiency for $\eta<0$, and the solid dots are the efficiency for $\eta>0$. The parameters $p 0$ and $p 1$ is the mean and width of the gaussian of the error function. $p 2$ is the normalization constant. 


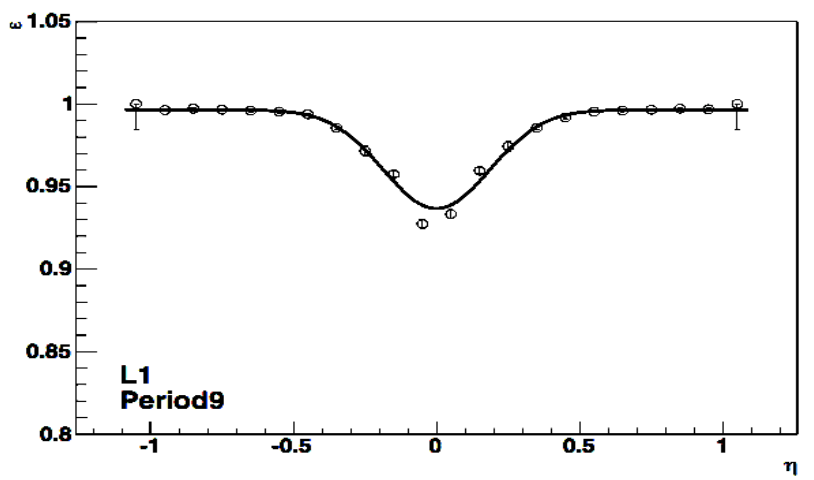

(a) L1_PT8

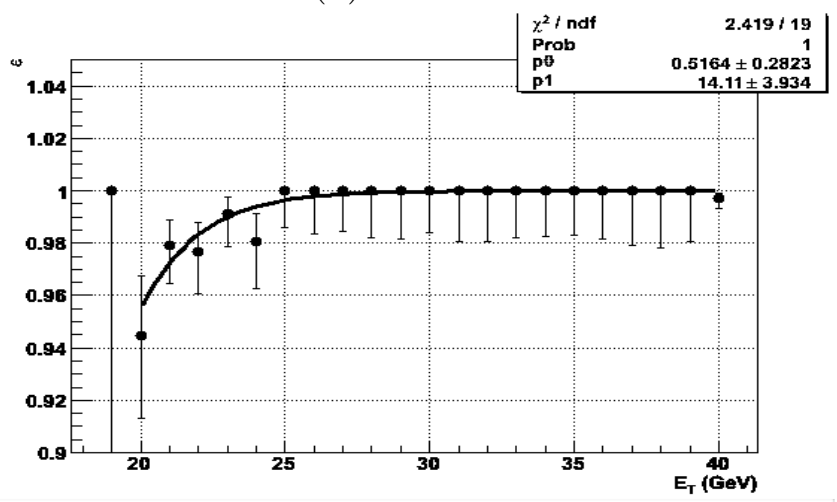

(b) $L 2 \_C E M 16$

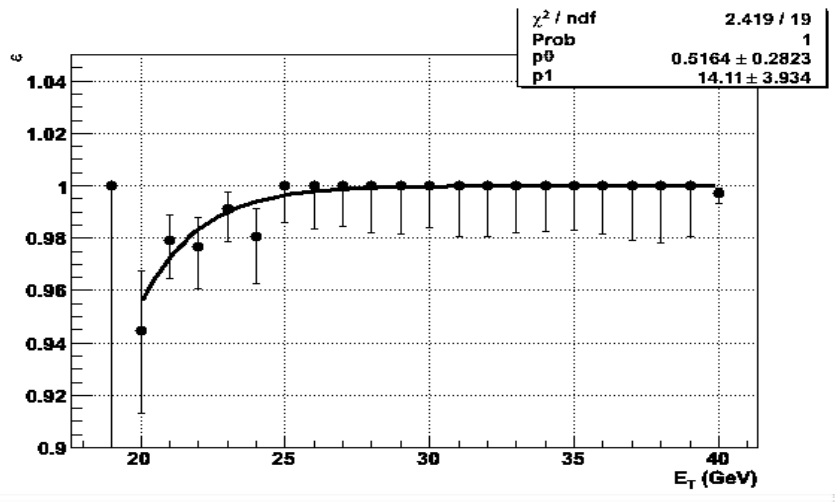

(c) L3_CENTRAL_ELECTRON18

Figure 3.18: ELECTRON_CENTRAL_18 trigger efficiency. The efficiency of $L 1 \_C E M 8$ is $\sim 100 \%$ in $E_{T}>20 \mathrm{GeV}$. The efficiencies shown in the plots are measured using the data collected from September, 2006 to November, 2006. 
where $E_{T}^{c}$ and $\eta^{c}$ are the transverse energy and pseudorapidity of the central electron and $E_{T}^{p}$ is the transverse energy of the plug electron. Both $E_{T}^{c}$ and $E_{T}^{p}$ are corrected by the energy scale. The $\mathrm{Z}(\mathrm{PP})$ topology only requires a $Z_{\_} N O T R A C K$ trigger. The event weighting factor for $\mathrm{Z}(\mathrm{PP})$ is defined as :

$$
w_{P P}\left(E_{T}^{1}, E_{T}^{2}\right)=\epsilon_{z}\left(E_{T}^{1}\right) \times \epsilon_{z}\left(E_{T}^{2}\right)
$$

where $E_{T}^{1}$ and $E_{T}^{2}$ are the corrected transverse energies of the electrons in the $\mathrm{Z}(\mathrm{PP})$ topology. The trigger efficiency is measured by the ratio of the weighted number of events to the total versus boson rapidity. Figure 3.19 shows the trigger efficiencies versus $y_{Z}$.

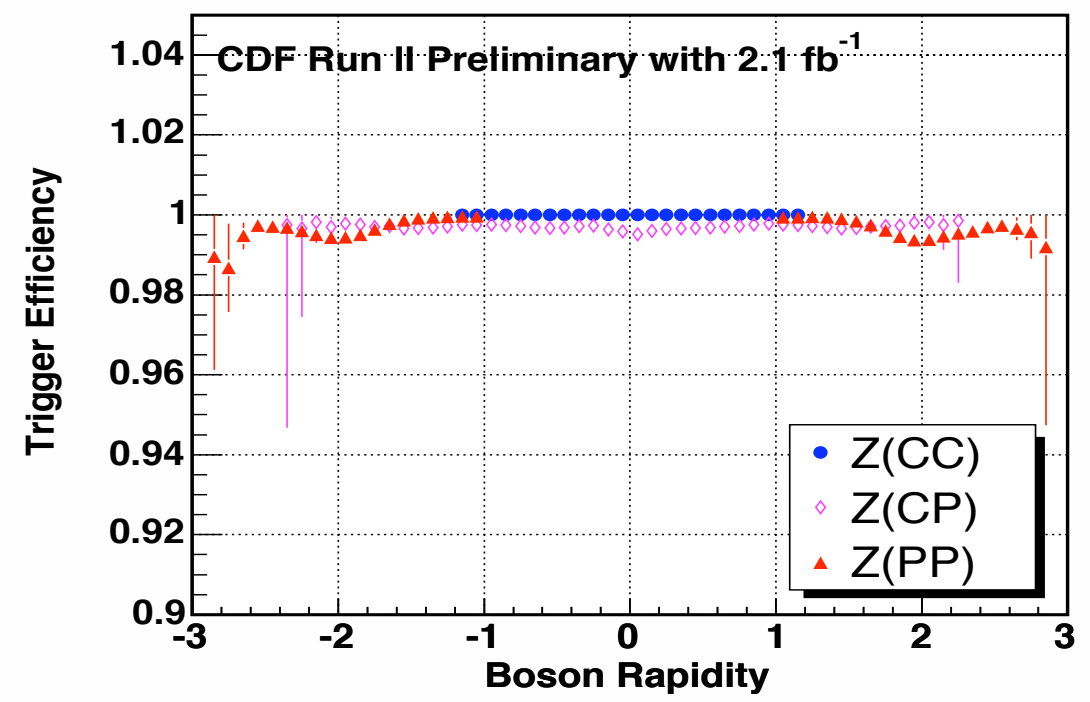

Figure 3.19: Trigger efficiency versus boson rapidity. The blue dots, magenta diamonds, and red triangles show the trigger efficiency for $\mathrm{Z}(\mathrm{CC}), \mathrm{Z}(\mathrm{CP})$, and $\mathrm{Z}(\mathrm{PP})$ events. respectively. 


\begin{tabular}{|c||c|c|c||}
\hline $\mathrm{y}$ & $\mathrm{CC}$ & $\mathrm{CP}$ & $\mathrm{PP}$ \\
\hline \hline 0.05 & 8514 & 566 & 0 \\
0.15 & 8142 & 1092 & 0 \\
0.25 & 7423 & 1960 & 0 \\
0.35 & 6605 & 2866 & 0 \\
0.45 & 5684 & 3758 & 0 \\
0.55 & 4909 & 4697 & 0 \\
0.65 & 3942 & 5428 & 0 \\
0.75 & 2947 & 6338 & 0 \\
0.85 & 1685 & 7341 & 0 \\
0.95 & 766 & 8301 & 0 \\
1.05 & 155 & 8742 & 24 \\
1.15 & 12 & 8691 & 221 \\
1.25 & 0 & 7510 & 751 \\
1.35 & 0 & 6125 & 1607 \\
1.45 & 0 & 4817 & 2484 \\
1.55 & 0 & 3505 & 3224 \\
1.65 & 0 & 2313 & 3785 \\
1.75 & 0 & 1280 & 3925 \\
1.85 & 0 & 613 & 3880 \\
1.95 & 0 & 223 & 3594 \\
2.05 & 0 & 51 & 2890 \\
2.15 & 0 & 13 & 2041 \\
2.25 & 0 & 0 & 1427 \\
2.35 & 0 & 0 & 856 \\
2.45 & 0 & 0 & 397 \\
2.55 & 0 & 0 & 169 \\
2.65 & 0 & 0 & 54 \\
2.75 & 0 & 0 & 14 \\
2.85 & 0 & 0 & 3 \\
2.95 & 0 & 0 & 0 \\
\hline \hline Total & 50784 & 86230 & 31346 \\
\hline & & & \\
\hline
\end{tabular}

Table 3.6: The number of events after selections in each topology. The background is not subtracted yet. 


\section{Chapter 4}

\section{Background}

After selection cuts, the background level for $Z / \gamma^{*}$ events in this analysis is relatively small. In particular, the tight central electron requirement for $\mathrm{Z}(\mathrm{CC})$ and $\mathrm{Z}(\mathrm{CP})$ events significantly reduces the background level in the sample. In contrast, the $\mathrm{Z}(\mathrm{PP})$ sample is expected to have more background. The requirement of a silicon phoenix track on at least one of the two electrons in the $\mathrm{Z}(\mathrm{PP})$ sample reduces the background to a manageable level .

The main sources of background in this measurement are jets from QCD processes. A QCD jet may be misidentified as an electron and included in the signal sample. In addition, there are real electrons in QCD jets that originate from bottom and charm particle decays. Dijet events are the main source of background because of the large QCD dijet cross section. We have developed the isolation extrapolation method that uses the data to directly measure the background level from QCD dijet events in the sample. In addition, we also measure the background level in an independent way by fitting the Z/Drell-Yan dielectron mass distribution.

The isolation extrapolation method determines the background level using one of the two electrons in the dielectron sample. This method is used to determine the 
background originating from QCD two jet events. However, background processes that have a hadron jet in one leg and a single isolated photon or electron in the other leg, are harder to measure using the isolation extrapolation technique.

A fit the Z/Drell-Yan dielectron mass distribution can be used to complement the isolation extrapolation method. Here, the side bands in the dielectron mass distribution on each side of the $\mathrm{Z}$ peak are used investigate the background level from all sources. This technique is sensitive to both, QCD background from dijet events as well as background from processes with one jet and an isolated electron or photon in the other leg. By combining these two independent methods we obtain a better estimation of the background.

Additional backgrounds from electroweak processes are relatively small and are estimated using a Monte Carlo simulation.

\subsection{Isolation Extrapolation Method}

\subsubsection{QCD Backgrounds}

The QCD background is determined directly from the data using the isolation extrapolation method. The isolation energy is defined as the energy contained in a $\Delta R=0.4$ cone outside of the electron shower. Electrons originating from real Z/Drell-Yan dielectron events are not expected to have nearby hadrons. The only additional particles nearby in real Z/Drell-Yan dilepton events are radiated photons from bremsstrahlung process (either internal or external). Therefore, the signal sample should include electrons with an isolation energy distribution which peaks at low isolation energy. In contrast, QCD jets include several hadrons, and a large fraction of QCD events are 


\section{Isolation Distribution}

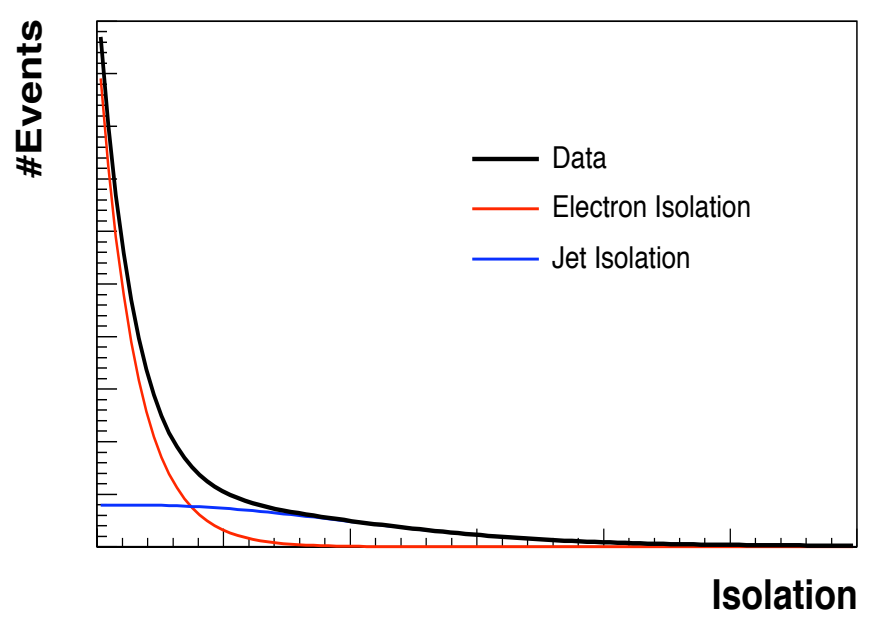

Figure 4.1: The isolation extrapolation method. The cartoon illustrates the isolation extrapolation method used to measure the QCD background. The signal (electron) and background (jet) shape templates are used to fit the isolation distribution in the data. The magnitude of the background is determined by from the fit parameters.

expected to have high isolation energy. Therefore, since background processes dominate the high isolation energy region, the QCD background level can be extrapolated from high isolation into the signal region by fitting the isolation distribution to a superposition of the expected isolation energy distribution for signal and background events. Figure 4.1 illustrates the isolation extrapolation method.

\subsubsection{Signal and Background Template}

The templates of the isolation energy (IsolationE) distribution for signal and background are obtained from signal and background dominated samples, respectively. The full dielectron sample which is used in the measurement is also used for the determination of the signal and background templates in order to avoid any sample 
bias.

The selection criteria for the signal and background dominated samples are summarized in Table 4.1. The two electromagnetic (EM) objects in the sample are split into a tagged and target leg. The target leg is used for the IsolationE fit, and the tagged leg is used for selecting signal dominated and background dominated samples. The target leg includes all electron ID cuts except for IsolationE. With tight electron ID cuts on the tagged leg, we select a signal dominated dielectron sample. We use anti-electron ID cuts on the tagged leg to select a QCD background dominated sample. For the background dominated sample we use the IsolationE and Had/EM variables of the tagged leg for selecting a sample of events originating from QCD dijets.

The initial signal dominated sample is not entirely pure, and is found to include a very small amount of residual QCD dijet background. This residual background is more easily observable at high IsolationE. The background fraction in the signal dominated sample at high IsolationE is determined by using a fit to the $\mathrm{Z}$ mass dielectron spectrum in the high IsolationE region. After this residual QCD dijet background is removed we are left with a pure signal dominated template.

The background dominated sample contains both QCD dijets and a small amount of $W \rightarrow e \nu+$ jets and $Z \rightarrow \tau \tau$ events. Electrons that originated from the decays of $\mathrm{W}$ bosons in $W \rightarrow e \nu+$ jets events are included in the target leg of the background dominated sample. Also, $\tau$ leptons originating from $\mathrm{Z}$ boson decays can decay to an electron (from one of the $\tau$ 's) and a hadron jet (from that other $\tau$ 's), and thus contribute to the initially selected QCD dijet background dominated sample. Therefore, a small number of real electrons is included in the background IsolationE shape from 
these two electroweak processes. The expected number of $W \rightarrow e \nu+$ jets and $Z \rightarrow \tau \tau$ events, normalized to the corresponding integrated luminosity, is removed from the background dominated sample yielding a pure QCD dijet background template. To estimate the expected amount of $W \rightarrow e \nu+$ jets and $Z \rightarrow \tau \tau$, simulated events from inclusive $W \rightarrow e \nu$ and $Z \rightarrow \tau \tau$ processes are used. The contribution of $Z \rightarrow \tau \tau$ events is negligible and does not affect the background distribution. However, events from the $W \rightarrow e \nu+$ jets process contribute to the low IsolationE region of the background sample, especially in the $\mathrm{Z}(\mathrm{CC})$ topology. To check the normalization factor of the $W \rightarrow e \nu+$ jets Monte Carlo, we use the MET (Missing $E_{T}$ ) distributions in the data to normalize $W \rightarrow e \nu+$ jets Monte Carlo sample. Figure 4.2 shows the MET distribution of the background sample and the normalized $W \rightarrow e \nu$ simulation sample in the $\mathrm{Z}(\mathrm{CC})$ region. At first, the normalization factor of the inclusive $W \rightarrow e \nu$ sample, NF1 is calculated to have the same integrated luminosity as the data. This normalization factor is applied to the $W \rightarrow e \nu$ events which pass the background sample selection cuts described in Table 4.1. The MET distribution normalized by NF1 in the inclusive $W \rightarrow e \nu$ simulation sample exceeds the data at high MET. Note that Z/Drell-Yan dilepton events populate the low MET region. Most of the events in the high MET region come from inclusive $W \rightarrow e \nu$ events. Therefore, we extract a new normalization factor (NF2) from the data such that the level of inclusive $W \rightarrow e \nu \mathrm{MC}$ events is the same as the data for $M E T>30 \mathrm{GeV}$. We apply this new NF2 normalization factor to the inclusive $W \rightarrow e \nu$ MC sample. Figure 4.3 and 4.4 show the MET distribution and MET vs. IsolationE scatter plot for the QCD dijet background dominated sample, and for the inclusive $W \rightarrow e \nu \mathrm{MC}$ sample normalized by NF2. After these electroweak contributions to the QCD background dominated 


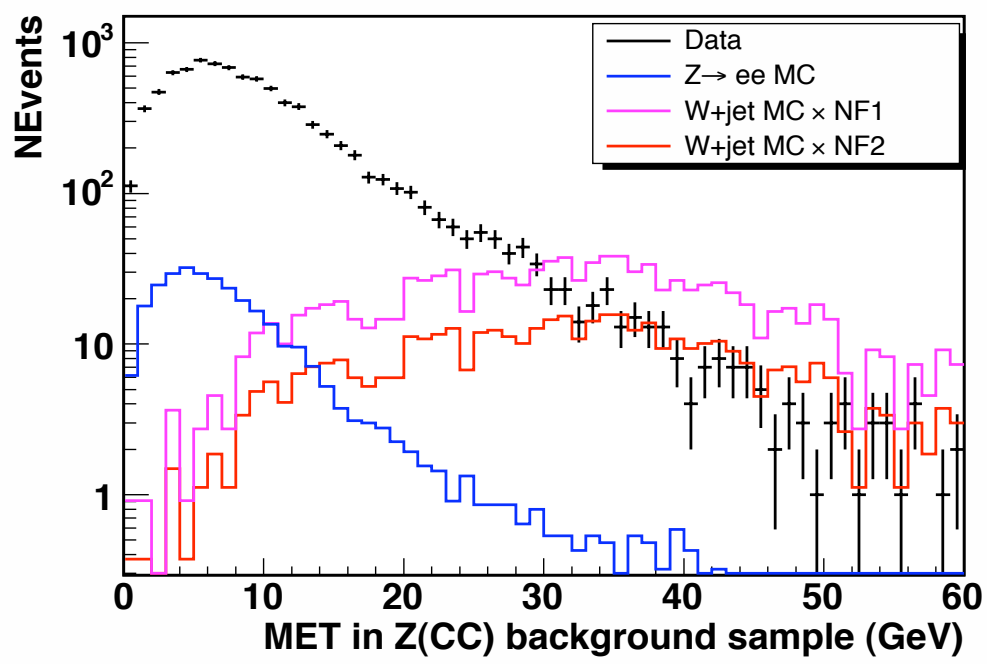

Figure 4.2: The MET distribution in the background sample. The scale factor, NF1 is calculated by comparing the integrated luminosity of MC with data. The scale factor $\mathrm{NF} 2$ is obtained requiring that the number of inclusive $W \rightarrow e \nu \mathrm{MC}$ events with $M E T>30 \mathrm{GeV}$ is in agreement with the data. The blue histogram is the normalized $Z \rightarrow$ ee $\mathrm{MC}$ events passing the background selection. The MET distribution of $Z \rightarrow e e \mathrm{MC}$ events peaks in low MET region $(\sim 5 \mathrm{GeV})$.

sample are removed, we are left with a pure QCD dijet background sample.

The electroweak background contribution from $W \rightarrow e \nu+$ jets and $Z \rightarrow \tau \tau$ processes to the final dielectron signal is determined separately using an independent Monte Carlo simulation.

\subsubsection{IsolationE Fit}

The magnitude of the QCD background is estimated by fitting the IsolationE distribution to a combination of signal and background. Since the IsolationE is corrected for the leakage of energy in the tower, it is over-corrected sometimes (from fluctuations) which leads to a negative IsolationE component. In the study, events with 
(a) Signal Dominated Sample - tagged leg requirements

\begin{tabular}{|c|c|c|}
\hline & For central electron & For plug electron \\
\hline Topology & tagged Leg & tagged Leg \\
\hline $\mathrm{Z}(\mathrm{CC})$ & Loose Central & N $/ \mathrm{A}$ \\
& Had $/$ Em $<0.05$ & \\
& IsolationE $<2$ & \\
\hline $\mathrm{Z}(\mathrm{CP})$ & Plug & Tight Central \\
& Had $/$ Em $<0.05$ & Had $/$ Em $<0.05$ \\
& IsolationE $<2$ & IsolationE $<2$ \\
\hline $\mathrm{Z}(\mathrm{PP})$ & $\mathrm{N} / \mathrm{A}$ & Plug \\
& \multicolumn{2}{|c|}{ Isad $/$ Em $<0.05$} \\
& $66<M_{e e}<116$ GeV $/ \mathrm{c}^{2}$ \\
\hline Mass & \multicolumn{2}{|c}{} \\
\hline
\end{tabular}

(b) Background Dominated Sample - tagged leg requirement

\begin{tabular}{|c|c|c|}
\hline & For central electron & For plug electron \\
\hline Topology & tagged Leg & tagged Leg \\
\hline $\mathrm{Z}(\mathrm{CC})$ & Loose Central & N $/ \mathrm{A}$ \\
& Had $/$ Em $>0.05$ & \\
& IsolationE $>5$ & \\
\hline $\mathrm{Z}(\mathrm{CP})$ & Plug & Loose Central \\
& Remove PEM $\chi_{3 \times 3}^{2}$ & - \\
& Had $/$ Em $>0.05$ & Had $/$ Em $<0.05$ \\
& IsolationE $>5$ & IsolationE $>5$ \\
\hline $\mathrm{Z}(\mathrm{PP})$ & $\mathrm{N} / \mathrm{A}$ & Plug \\
& \multicolumn{2}{|c|}{$66<M_{e e}<116$ GeV $/ c^{2}$} \\
& \multicolumn{2}{|c|}{ Remove PEM $\chi_{3 \times 3}^{2}$} \\
& \multicolumn{2}{|c|}{ IsolationE $>0.05$} \\
\hline Mass &
\end{tabular}

Table 4.1: The signal and background selection criteria for extracting the signal dominated and background dominated templates. The $\mathrm{Had} / \mathrm{Em}$ and Isolation E requirements are redefined in the table. One of the two electrons in the $\mathrm{Z}$ boson/dielectron event sample is defined as the target and and the other electron is defined as the tagged leg, respectively. The IsolationE of the target leg is used in isolation distribution fit. The target leg is required to have the same cuts as the standard analysis selection cuts, except for IsolationE requirement. 

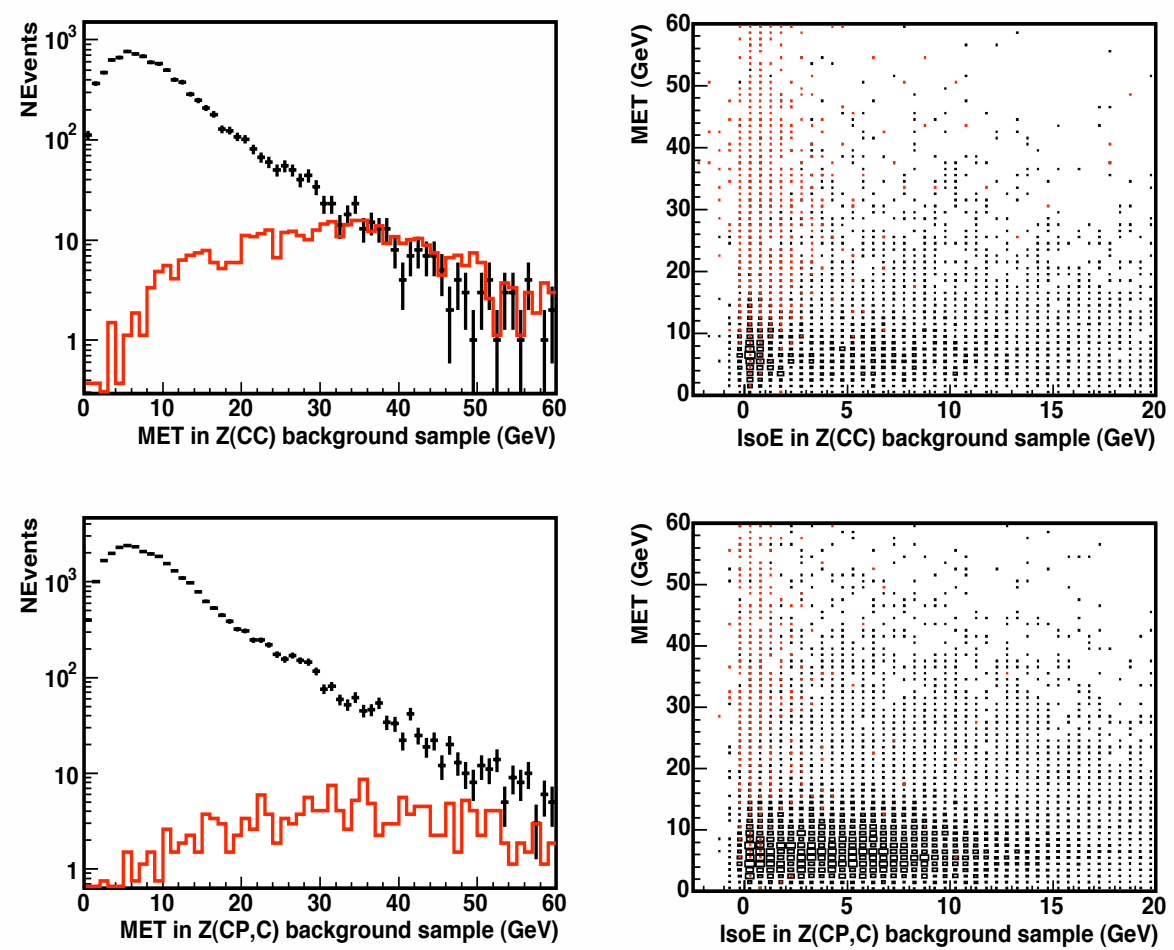

Figure 4.3: The MET distribution of the background sample for central electrons. The cross points are the background sample, and the red histograms are normalized inclusive $\mathrm{W}$ events from the MC. 

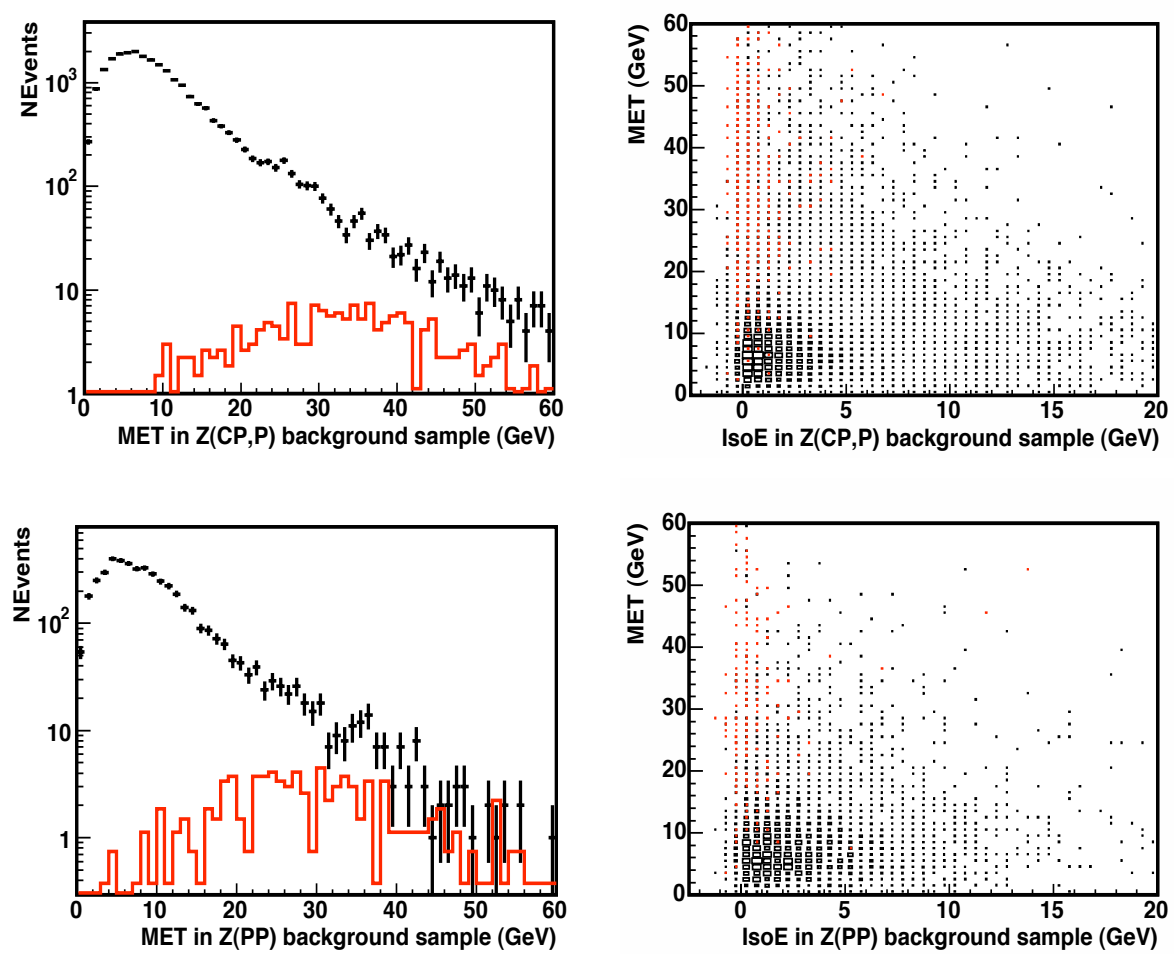

Figure 4.4: The MET distribution of the background sample for plug electrons. The cross points are the background sample, and the red histograms are normalized inclusive $\mathrm{W}$ events from the MC. 
negative IsolationE are included in first positive bin of the IsolationE distribution. The low IsolationE region is dominant by signal from real Z/Drell Yan dielectrons. We do not fit the low IsolationE region because it is difficult to model and we just count events there. Only the background is modeled in this region. In the Isolation extrapolation method, the highest sensitivity in the determination of the background level is in the high IsolationE region. The background level at high IsolationE is large, and the background contribution can be clearly distinguished from signal events. To determine the fit range in IsolationE, we vary the starting point of the fit and compare the $\chi^{2}$ in the high IsolationE region, $E_{i s o}>4 \mathrm{GeV}$. Table 4.2 shows the $\chi^{2}$ for fit to the $E_{i s o}$ distributions with different starting points. The $\chi^{2}$ in each case becomes stable for $E_{i s o}>2 \mathrm{GeV}$. Therefore, we use the $E_{i s o}>2 \mathrm{GeV}$ region of the IsolationE distribution to determine the level of the QCD background.

The selection bias for the background sample is larger than for the signal sample. In particular, the requirement of the anti-electron selection cuts on the tagged leg in the plug can bias the background template distribution. One of anti-electron selection criteria $\left(E_{i s o}>5 \mathrm{GeV}\right)$ on the tagged leg in the plug suppresses the acceptance in the $|\eta|>2.3$ region, where the size of the calorimeter towers in $\eta-\phi$ is large. This biases the kinematics of the selected background events. However, plug electrons which are associated with a track are less sensitive to the anti-electron cut because the contribution from high $\eta$ electrons is already suppressed by the tracking inefficiency. Therefore, background samples with an anti-electron cut on the central leg in the $\mathrm{Z}(\mathrm{CP})$ topology, or on the plug leg with a track in the $\mathrm{Z}(\mathrm{PP})$ topology are used to reduce the selection bias. In the IsolationE fit we select the target leg to be the plug leg in $\mathrm{Z}(\mathrm{CP})$ events, and to be the non-track leg in $\mathrm{Z}(\mathrm{PP})$ events. This provides a 
(a) Z(CC) Topology

\begin{tabular}{|c||c|c|}
\hline Fit Region & $R_{b}$ in $E_{\text {iso }}<4$ & $\chi^{2}$ \\
\hline$E_{\text {iso }}>0$ & $0.0035 \pm 0.0003$ & 37.91 \\
$E_{\text {iso }}>1$ & $0.0028 \pm 0.0002$ & 14.52 \\
$E_{\text {iso }}>2$ & $0.0024 \pm 0.0003$ & 8.52 \\
$E_{\text {iso }}>3$ & $0.0023 \pm 0.0003$ & 7.20 \\
$E_{\text {iso }}>4$ & $0.0022 \pm 0.0003$ & 7.14 \\
$E_{\text {iso }}>5$ & $0.0023 \pm 0.0004$ & 7.26 \\
\hline
\end{tabular}

(b) Z(CP) Topology

\begin{tabular}{|c||c|c||c|c|}
\hline \multicolumn{1}{|c||}{} & \multicolumn{2}{c||}{ Central Leg } & \multicolumn{2}{c|}{ Plug Leg } \\
\hline Fit Region & $R_{b}$ in $E_{\text {iso }}<4$ & $\chi^{2}$ & $R_{b}$ in $E_{\text {iso }}<4$ & $\chi^{2}$ \\
\hline$E_{\text {iso }}>0$ & $0.0098 \pm 0.0003$ & 42.18 & $0.0191 \pm 0.0013$ & 49.67 \\
$E_{\text {iso }}>1$ & $0.0088 \pm 0.0003$ & 17.27 & $0.0148 \pm 0.0012$ & 24.97 \\
$E_{\text {iso }}>2$ & $0.0085 \pm 0.0004$ & 12.65 & $0.0118 \pm 0.0014$ & 15.43 \\
$E_{\text {iso }}>3$ & $0.0083 \pm 0.0004$ & 11.27 & $0.0101 \pm 0.0015$ & 11.54 \\
$E_{\text {iso }}>4$ & $0.0083 \pm 0.0006$ & 11.24 & $0.0087 \pm 0.0024$ & 9.69 \\
$E_{\text {iso }}>5$ & $0.0082 \pm 0.0007$ & 11.26 & $0.0098 \pm 0.0068$ & 12.11 \\
\hline
\end{tabular}

(c) Z(PP) Topology

\begin{tabular}{|c||c|c||c|c|}
\hline \multicolumn{1}{|c||}{} & \multicolumn{2}{c||}{ Phoenix Leg } & \multicolumn{2}{c|}{ Plug Leg } \\
\hline Fit Region & $R_{b}$ in $E_{\text {iso }}<4$ & $\chi^{2}$ & $R_{b}$ in $E_{\text {iso }}<4$ & $\chi^{2}$ \\
\hline$E_{\text {iso }}>0$ & $0.0222 \pm 0.0017$ & 35.05 & $0.0461 \pm 0.0032$ & 28.73 \\
$E_{\text {iso }}>1$ & $0.0178 \pm 0.0017$ & 20.36 & $0.0374 \pm 0.0035$ & 17.03 \\
$E_{\text {iso }}>2$ & $0.0140 \pm 0.0019$ & 14.40 & $0.0304 \pm 0.0040$ & 12.59 \\
$E_{\text {iso }}>3$ & $0.0120 \pm 0.0021$ & 13.45 & $0.0266 \pm 0.0055$ & 11.26 \\
$E_{\text {iso }}>4$ & $0.0121 \pm 0.0027$ & 13.48 & $0.0244 \pm 0.0076$ & 10.83 \\
$E_{\text {iso }}>5$ & $0.0110 \pm 0.0043$ & 14.99 & $0.0283 \pm 0.0147$ & 12.63 \\
\hline
\end{tabular}

Table 4.2: The background rate and $\chi^{2}$ in each fit range. $R_{b}$ is the background rate in the $E_{i s o}<4$ region, and $\chi^{2}$ is calculated for the $E_{i s o}>4$ region. 
more reliable estimate of the background because the background sample for in these cases is less sensitive to bias from the anti-electron selection criteria.

To determine the background fraction in the sample, the IsolationE distribution is fit to the sum of signal and background templates. For $\mathrm{Z}(\mathrm{CC})$ events, both legs are are used to estimate the background level. For $\mathrm{Z}(\mathrm{CP})$ and $\mathrm{Z}(\mathrm{PP})$ events, we divide the events into two-track and one-track categories. For the $\mathrm{Z}(\mathrm{CP})$ topology, the central electron is always associated with a track. Therefore, for $\mathrm{Z}(\mathrm{CP})$ events, the plug electron determines if the event belongs in the two-track or one-track category. Two-track events are expected to have a small background fraction because the track requirements on both legs greatly reduces the background from photons. In contrast, one-track events are expected to have a larger background contamination than twotrack events. Specifically, the $\gamma+$ jet QCD background only contributes to the onetrack category, when a photon is selected as a no-track electron leg. Figure 4.5 shows the plug isolationE shape difference between the track-leg and the no-track leg in the $\mathrm{Z}(\mathrm{CP})$ background sample. The IsolationE distribution for no-track legs shows an excess at very low IsolationE. This excess originates from photons from the QCD $\gamma+j e t$ process. Therefore, we consider QCD $\gamma+j e t$ events as an additional source of background in the one-track category.

One-track events can be separated into one-track events from which the no-track leg is in the silicon fiducial volume, and events for which the no-track leg is outside the silicon fiducial (non-silicon fiducial) volume and no track is expected. Therefore, we separate the $\mathrm{Z}(\mathrm{CP})$ and $\mathrm{Z}(\mathrm{PP})$ events into three categories. These are two-track events, one-track events with a no-track leg in the silicon detector fiducial volume, and one-track events with a no-track leg in the non-silicon detector fiducial volume. 
When both legs have a track in the Z(PP) topology, the two legs are combined to determine the magnitude of the background from the fit to the IsolationE distribution. For one-track events in silicon fiducial region, the track and no-track legs are fitted separately. The one-track background sample for the non-silicon fiducial region is of low statistics. Therefore, for this sample, only the track leg is used in the IsolationE fit. Figures $4.6,4.7,4.8,, 4.9$ and 4.10 shows the fit results in each topology. Table 4.3 summarizes the background fractions. The central leg in $\mathrm{Z}(\mathrm{CP})$ events yields a different background level than the plug leg when the IsolationE fit is done. However, the background sample for the case for which the central electron leg is used as the target leg in the fit has larger bias from the anti-electron cut on the plug leg than the other way around. This results in different QCD $\gamma+$ jet background fractions in the sample. For the $\mathrm{Z}(\mathrm{PP})$ topology and the one track case, there is a difference between the result using the track leg and the no-track leg as a target leg in the fit for the same reason. To reduce the sample bias in the background template, only events with the anti-electron selection on the central leg in the $\mathrm{Z}(\mathrm{CP})$ sample, and on the track leg in the $\mathrm{Z}(\mathrm{PP})$ sample, are used to obtain the background template.

Table 4.4 summarizes the background fraction in the high isolationE region of the signal sample that is used subtract the background from the signal template. The background fraction in the template is obtained by using a fit to the Z/Drell-Yan dielectron mass distribution. 


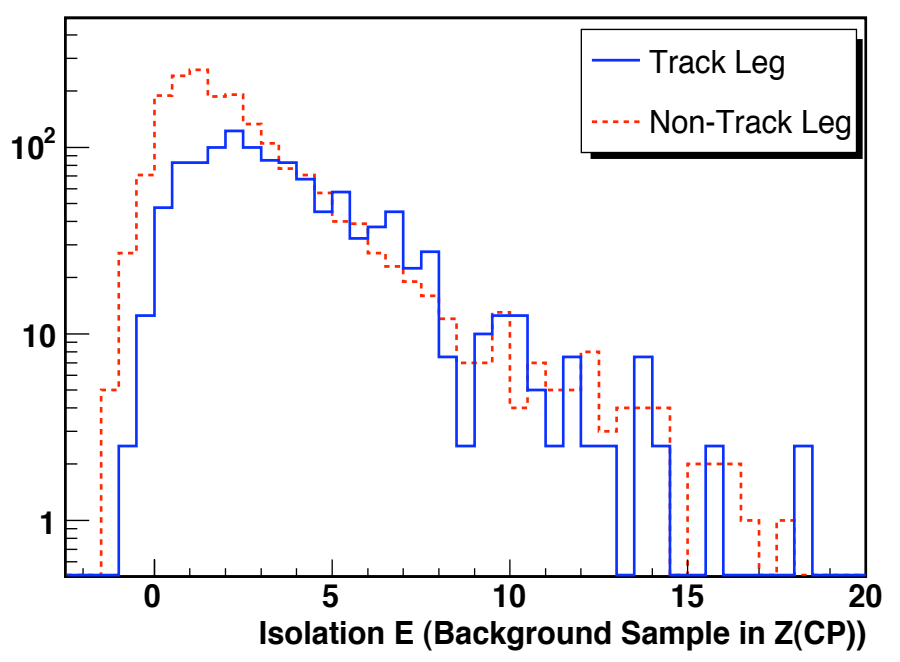

Figure 4.5: Plug IsolationE of track and no-track legs in the $\mathrm{Z}(\mathrm{CP})$ background sample. Photons from the QCD $\gamma+$ jet process contribute to events at low IsolationE with a no-track leg. Both histograms are normalized to have same number of events for $E_{\text {iso }}>5 \mathrm{GeV}$.

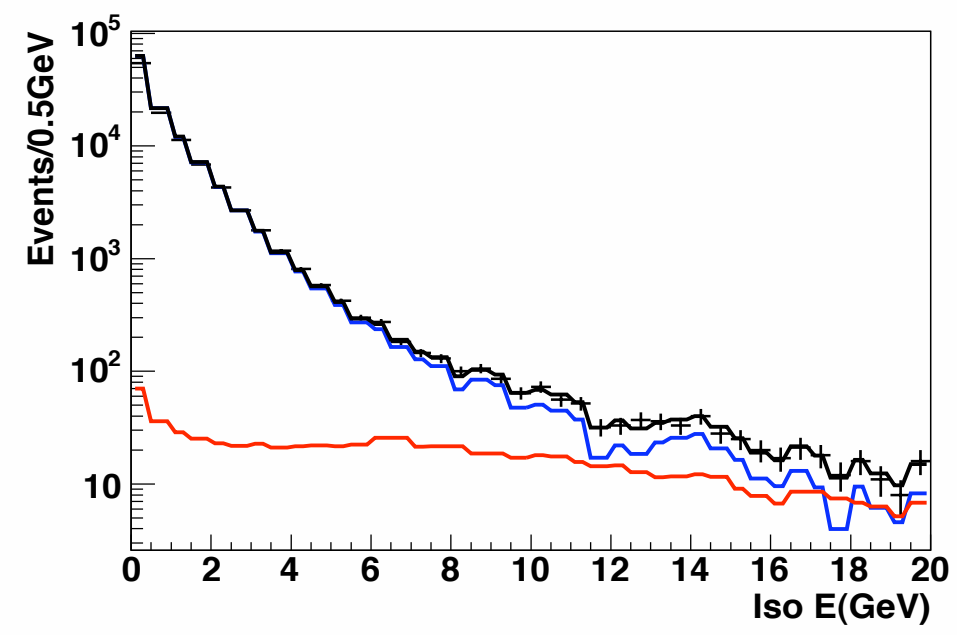

Figure 4.6: IsolationE fit for the central electron of $\mathrm{Z}(\mathrm{CC})$ events. 


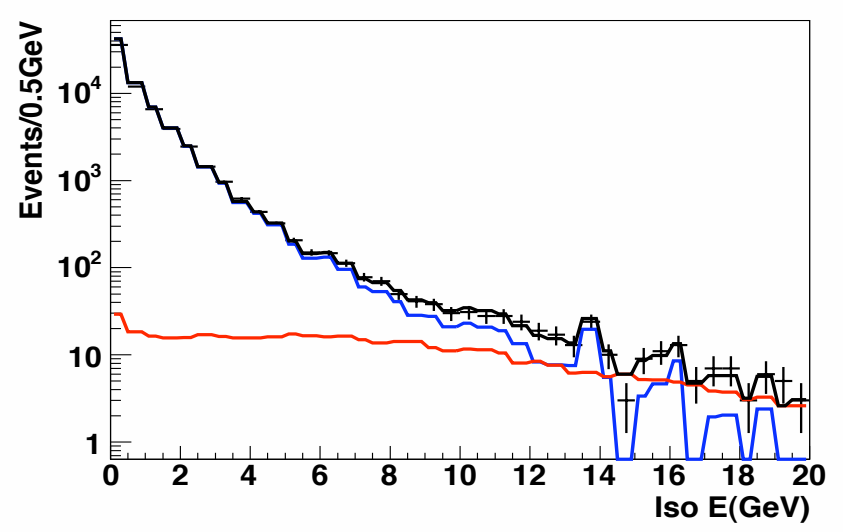

(a) 2 track

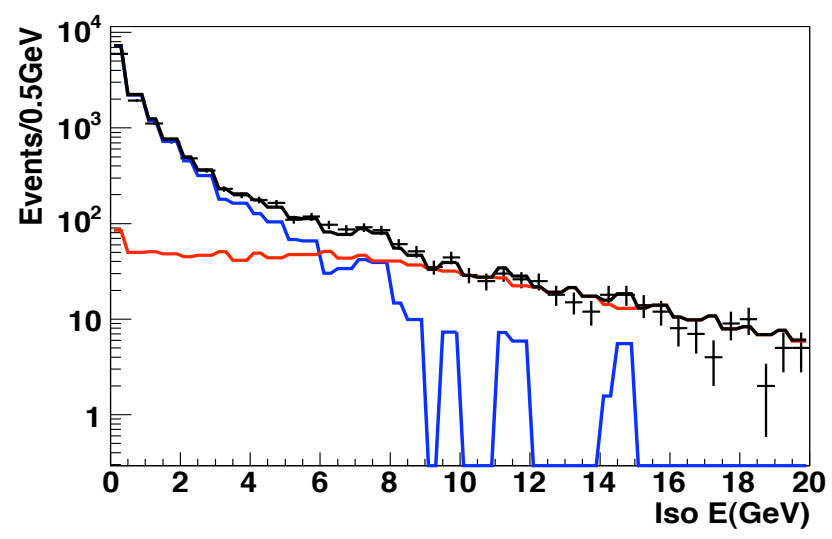

(b) 1 track and no-track leg(plug leg) in silicon fiducial volume

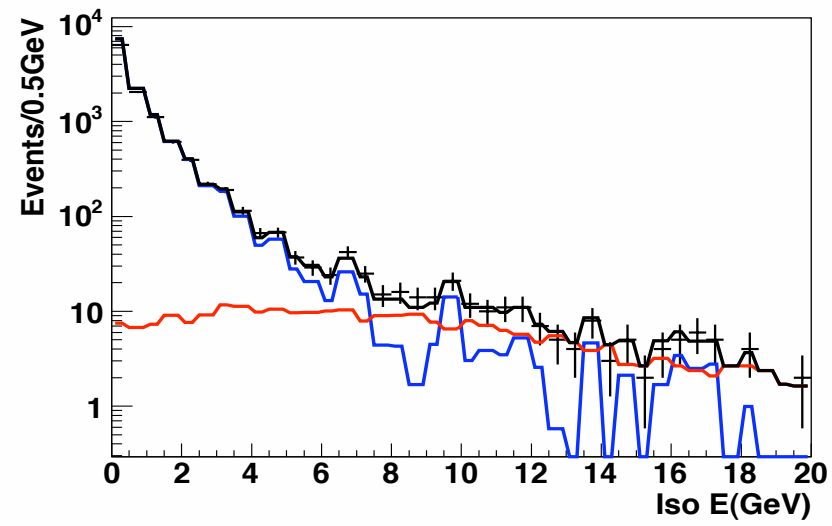

(c) 1 track and no-track leg(plug leg) in non-silicon fiducial volume

Figure 4.7: IsolationE fit for the central electrons of $\mathrm{Z}(\mathrm{CP})$ events. 


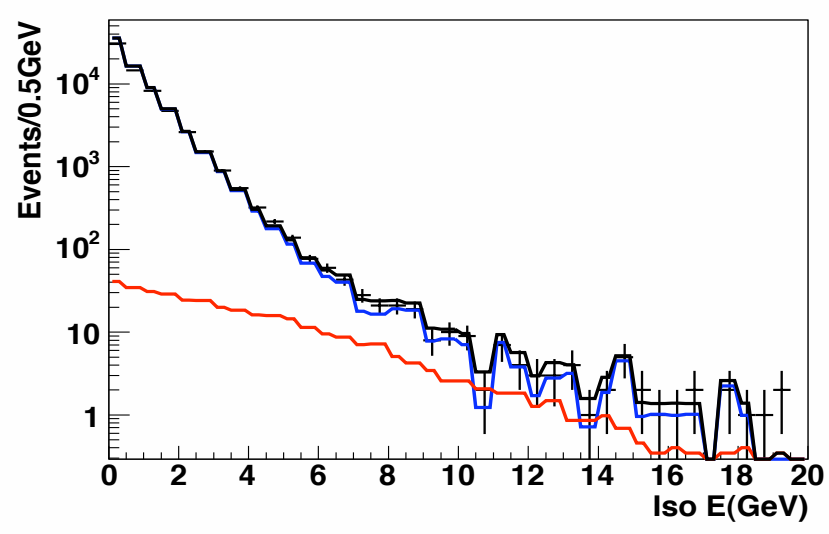

(a) 2 track

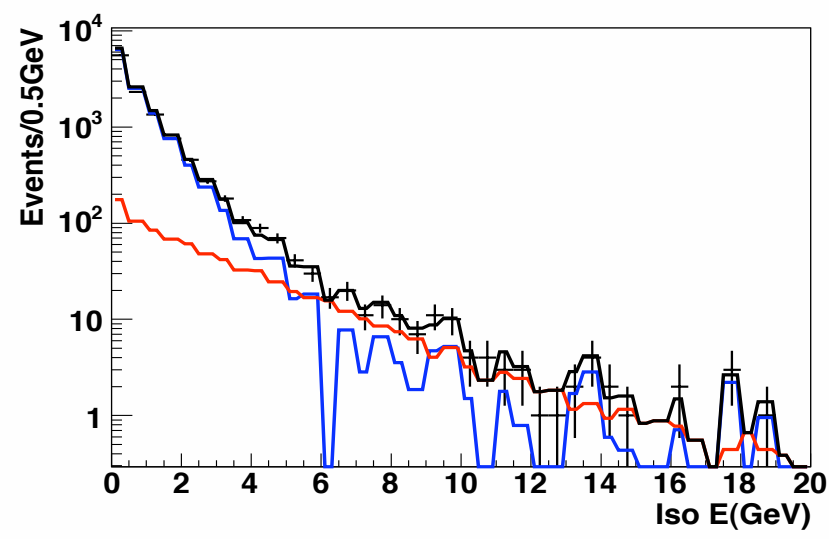

(b) 1 track and no-track leg(plug leg) in silicon fiducial volume

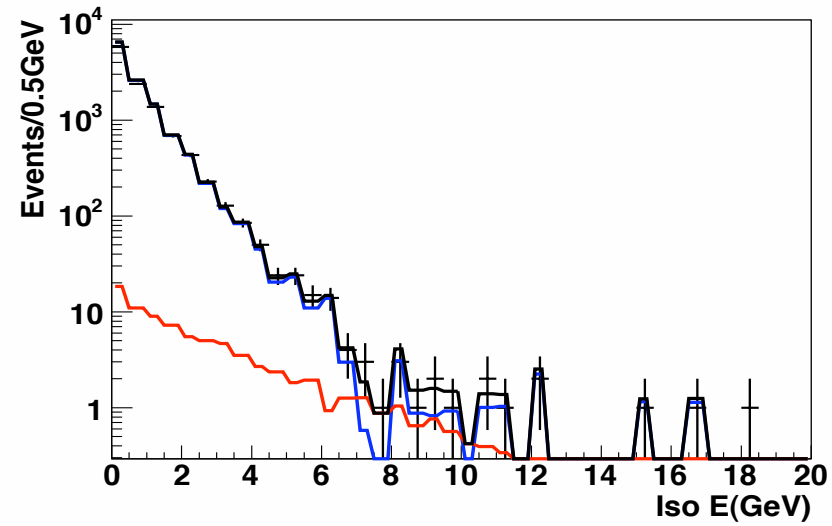

(c) 1 track and no-track leg(plug leg) in non-silicon fiducial volume

Figure 4.8: IsolationE fit for the plug electrons of $\mathrm{Z}(\mathrm{CP})$ events. 


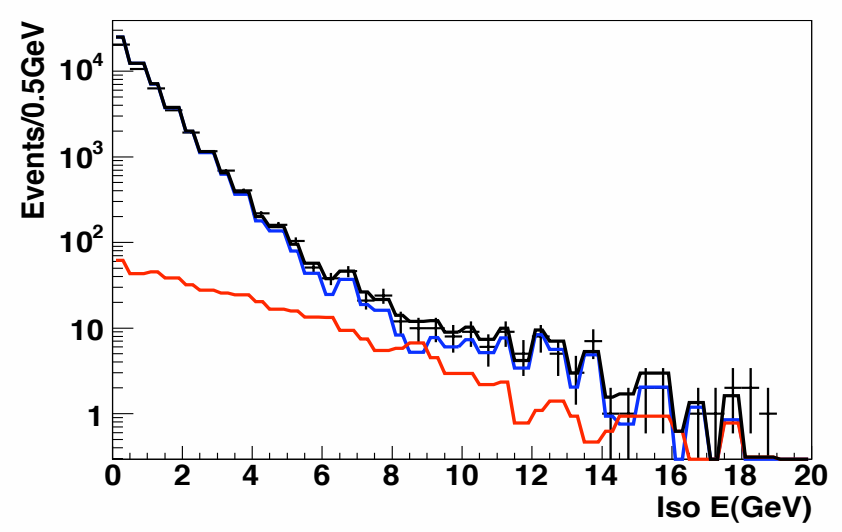

(a) 2 track

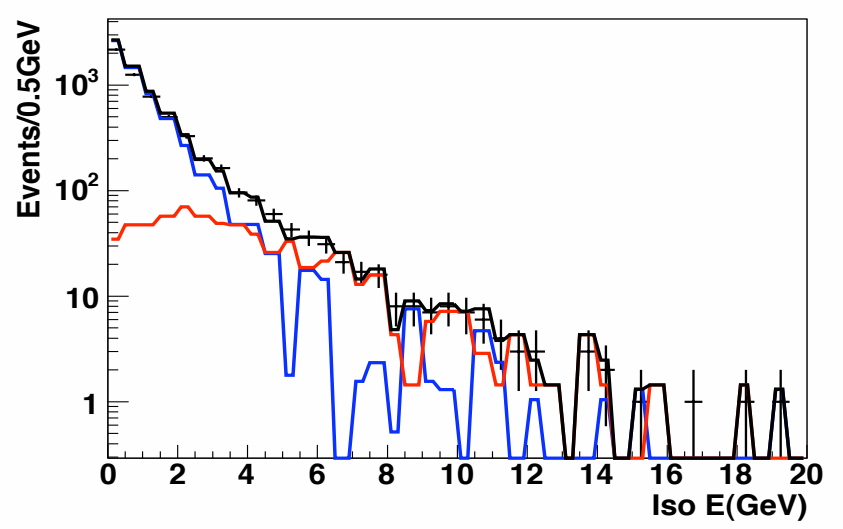

(b) 1 track and no-track leg in silicon fiducial volume

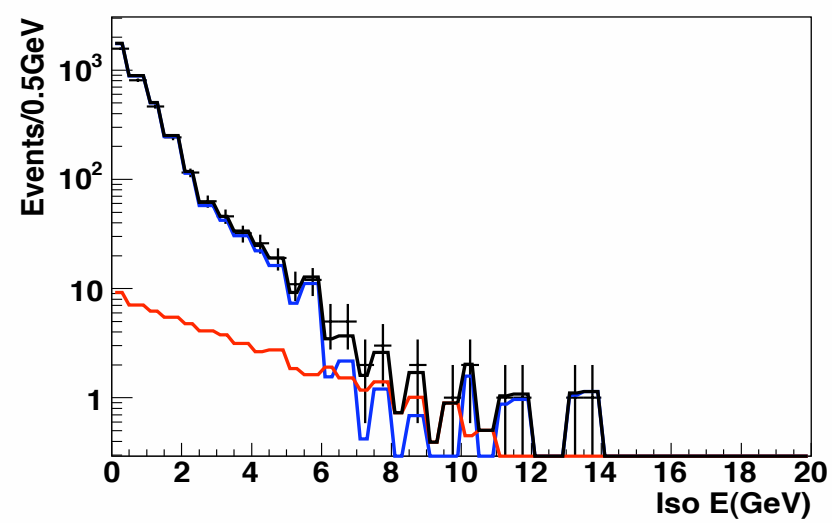

(c) 1 track and no-track leg in non-silicon fiducial volume

Figure 4.9: IsolationE fit for plug electrons with a track in $\mathrm{Z}(\mathrm{PP})$ events. 


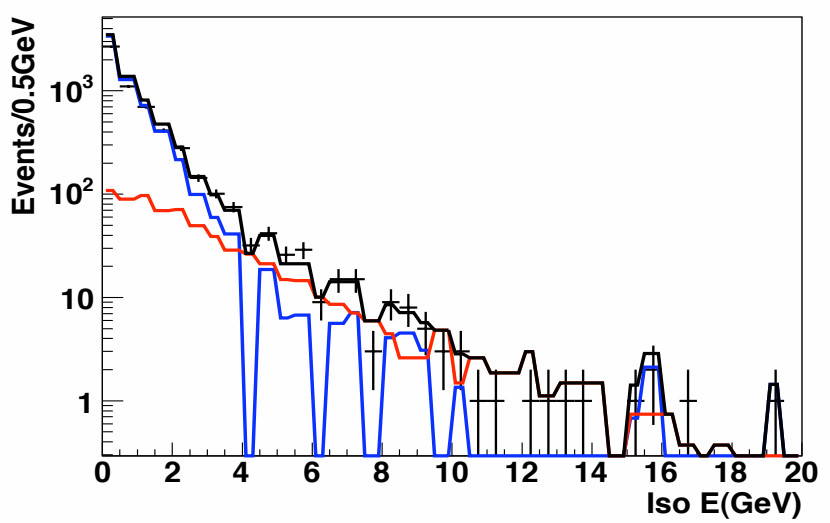

(a) 1 track in silicon fiducial volume

Figure 4.10: IsolationE fit of the plug electrons using the no-track leg in $\mathrm{Z}(\mathrm{PP})$ events (Only one track events).

\begin{tabular}{|c||c|l||c|}
\hline Topology & Target Leg & Category & Background Rate \\
\hline $\mathrm{Z}(\mathrm{CC})$ & central & & $0.0024 \pm 0.0003$ \\
\hline $\mathrm{Z}(\mathrm{CP})$ & central & 2 track & $0.0022 \pm 0.0003$ \\
& & 1 track + fid & $0.0378 \pm 0.0025$ \\
& & 1 track + nfid & $0.0063 \pm 0.0008$ \\
\hline $\mathrm{Z}(\mathrm{CP})$ & plug & 2 track & $0.0036 \pm 0.0008$ \\
& & 1 track + fid & $0.0558 \pm 0.0084$ \\
& & 1 track + nfid & $0.0058 \pm 0.0026$ \\
\hline Z(PP) & phoenix & 2 track & $0.0067 \pm 0.0012$ \\
& & 1 track + fid & $0.0747 \pm 0.0093$ \\
& & 1 track + nfid & $0.0143 \pm 0.0060$ \\
\hline Z(PP) & plug & 1 track + fid & $0.1002 \pm 0.0166$ \\
\hline
\end{tabular}

Table 4.3: The background fractions obtained using the isolation extrapolation method. The "fid"("nfid") means that the plug electron which does not have a matched track is in the silicon fiducial region(the non-silicon fiducial region). 


\begin{tabular}{|c||c|l||c|}
\hline Topology & Target Leg & Category & Background Rate \\
\hline $\mathrm{Z}(\mathrm{CC})$ & central & & $0.1055 \pm 0.0106$ \\
\hline $\mathrm{Z}(\mathrm{CP})$ & central & 2 track & $0.2796 \pm 0.0487$ \\
& & 1 track + fid & $0.9291 \pm 0.0733$ \\
& & 1 track + nfid & $0.6487 \pm 0.1022$ \\
\hline $\mathrm{Z}(\mathrm{CP})$ & plug & 2 track & $0.0816 \pm 0.0212$ \\
& & 1 track + fid & $0.4534 \pm 0.0839$ \\
& & 1 track + nfid & $0.0666 \pm 0.0509$ \\
\hline $\mathrm{Z}(\mathrm{PP})$ & phoenix & 2 track & $0.1116 \pm 0.0266$ \\
& & 1 track + fid & $0.6816 \pm 0.0678$ \\
& & 1 track + nfid & $0.3504 \pm 0.0868$ \\
\hline $\mathrm{Z}(\mathrm{PP})$ & plug & 1 track + fid & $0.7624 \pm 0.1077$ \\
\hline
\end{tabular}

Table 4.4: The background fraction in high IsolationE region of the signal sample. The background rate in the central leg of $\mathrm{Z}(\mathrm{CP})$ events is measured using the $E_{\text {iso }}>7 \mathrm{GeV}$ region, and the background rates in other categories are measured using the $E_{i s o}>$ $4 \mathrm{GeV}$ region. All background rates in the signal template are obtained by fitting the $\mathrm{Z} /$ Drell-Yan dielectron mass distribution. The initial signal template is corrected for this residual background to obtain a pure signal template. The "fid" ("nfid") means that the plug electron which does not have a matched track is in the silicon fiducial region(the non-silicon fiducial region).

\subsection{The Z/Drell-Yan dielectron Mass Fit Method}

The background fraction in the data is also estimated by fitting the Z/Drell-Yan dielectron mass distributions. Here a signal template for the dielectron mass distribution is obtained from the $Z \rightarrow e e$ MC sample, which is tuned to agree with data for the energy calibrations and efficiencies. The background template is obtained from the background event sample which is also used for the isolation extrapolation method. These selection criteria are described in Table 4.1. To reduce the sample bias in the background template, only events with the anti-electron selection on the central leg in the $\mathrm{Z}(\mathrm{CP})$ sample, and on the track leg in the $\mathrm{Z}(\mathrm{PP})$ sample, are used 
in the determination of the background template.

The Z/Drell-Yan dielectron events for which both electrons have associated tracks do not include QCD $\gamma+$ jet events because the track requirement removes any process which is associated with a photon. However, for events in which only one electron is associated with a track, the background from the $\gamma$ from the QCD $\gamma+$ jet process is not rejected. In this case, the no-track leg of the event may be a photon. Figure 4.11 shows the $|\eta|$ distribution of electrons without a track in the dielectron $Z \rightarrow e e$ data sample, compared to the $Z \rightarrow e e \mathrm{MC}$, as well as the $|\eta|$ distribution from the QCD $\gamma+$ jet MC samples. We separate the no-track data sample into no-track electrons in the silicon tracking fiducial volume, and no-track electrons outside the silicon tracking fiducial volume (non-silicon events). No-track electrons from real Z/Drell-Yan dielectron events can only originate from a tracking inefficiency. Since the tracking efficiency in the low $\eta$ region is high, there are fewer Z/Drell-Yan no-track events at low $\eta$. On the other hand, since the photon from the QCD $\gamma+$ jet process does not have a track, the $\eta$ distribution of the photon follows its acceptance into the no-track and track fiducial regions. Therefore, photons from the QCD $\gamma+$ jet process are more clearly seen in the data in the low $\eta$ region where its acceptance is large.

The background sample obtained using the anti-electron selection on one of the legs is a QCD di-jet dominant sample. This sample is used to get the shape of the mass distribution of the background from QCD dijet events. However, the antielectron selection introduces a bias in the sample and removes some of the QCD $\gamma+$ jet background. This is true in the 1-track case if the photon is part of the target leg. It is hard to measure the QCD $\gamma+$ jet background from fitting the IsolationE distribution, since if the photon is in the target leg, it is in general isolated, and 
appears as an electron from a Z/Drell-Yan dielectron event. The method, which uses a fit to the Z/Drell-Yan mass distribution (as described below), can be used to measure both sources of background. The side band region around the $\mathrm{Z}$ mass distribution include both the QCD dijet and the QCD $\gamma+$ jet (and a small number of electroweak background events).

\subsubsection{Mass distribution of QCD $\gamma+$ jet events}

To obtain the shape of the mass distribution of the background originating from QCD $\gamma+$ jet events, we simulate the QCD $\gamma+$ jet process with a Monte Carlo. This simulation does not include detector simulation. From this Monte Carlo, we obtain the slope of the mass distribution of the QCD $\gamma+$ jet background. The variables available in the Monte Carlo sample of QCD $\gamma+$ jet events include all the generated parton level information. However, we need to know the relationship between the energy of the final state recoil parton that fragments into the jet, and and energy of the electron-like object (e.g. an isolated neutral pion in the jet) which mimics an electron in the jet. In this study, we assume that the parton fragments into an electron-like object and other remaining particles ("stuff"). Both the electron-like object (e) in the jet and the photon are required to have $E_{T}>20 \mathrm{GeV}$ for $\mathrm{Z}(\mathrm{CP})$ events, and $E_{T}>25 \mathrm{GeV}$ for $\mathrm{Z}(\mathrm{PP})$ events. To get a realistic QCD $\gamma+j e t$ mass distribution, we consider the fragmentation energy fraction $(z)$ which is defined as the energy fraction of final state parton that is carried by the electron-like isolated object in the jet. parton $(z \leq 1.0)$.

$$
E_{T}(e)=z \times E_{T}(\text { parton })>20,25 \mathrm{GeV}(z \leq 1.0)
$$



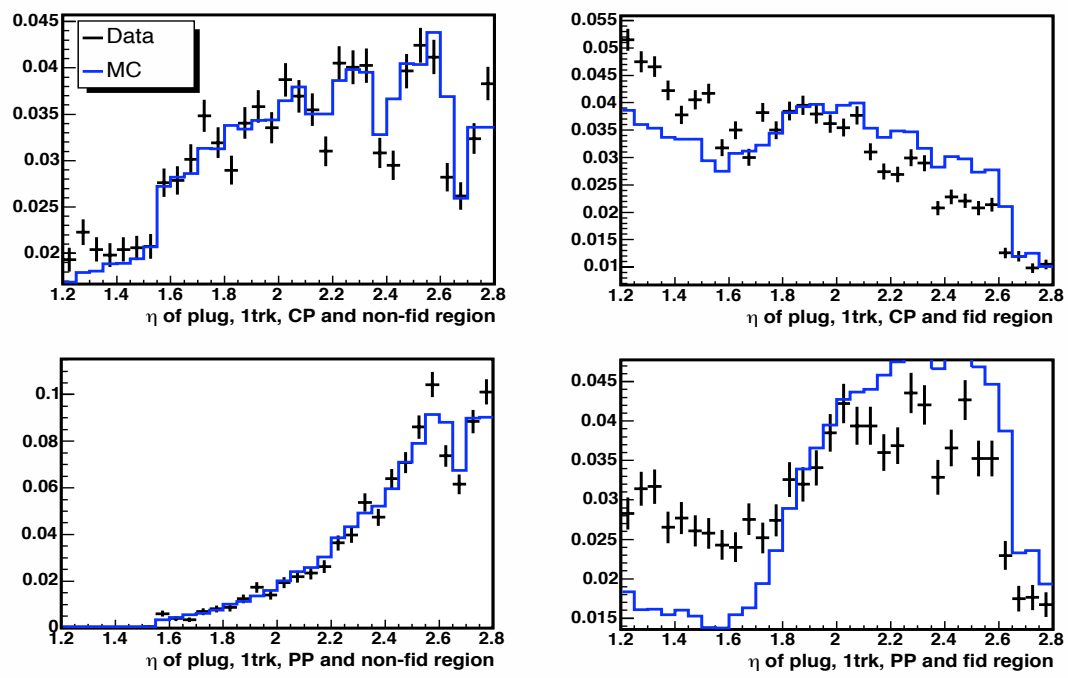

(a) Data vs. $Z \rightarrow e e \mathrm{MC}$ sample
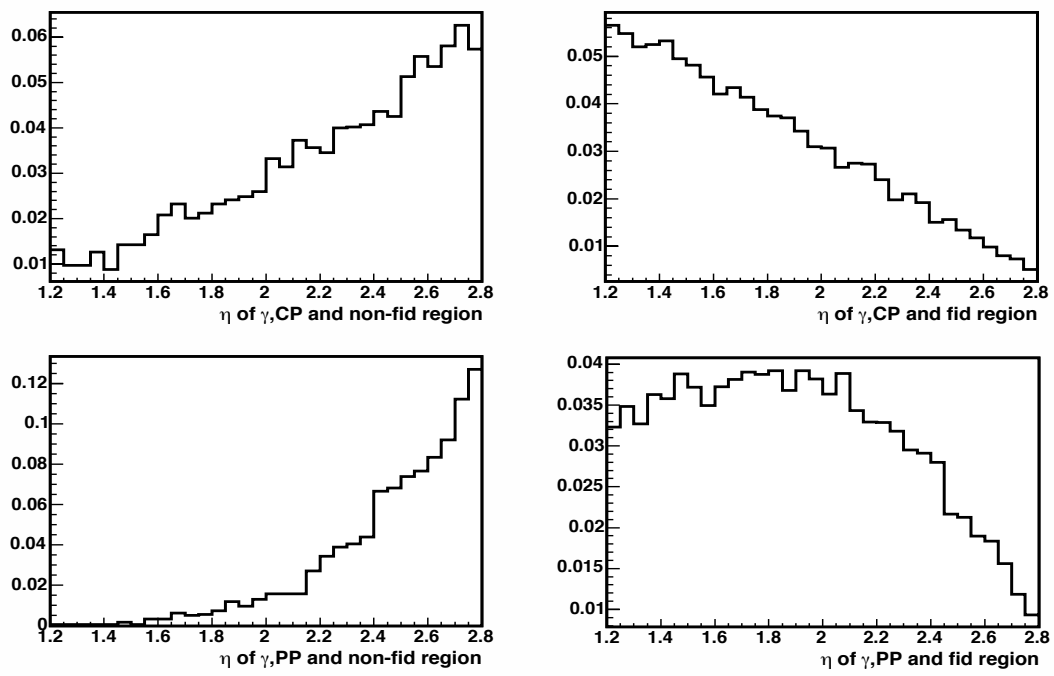

(b) $\gamma+$ jet MC sample

Figure 4.11: The $\eta$ distribution of plug electrons without a track. (a) The top two plots show the $\eta$ distribution of plug electrons in $\mathrm{Z}(\mathrm{CP})$ events in the data compared to $Z \rightarrow e e \mathrm{MC}$. The bottom two plots show the $\eta$ distribution of plug electrons in $\mathrm{Z}(\mathrm{PP})$ events. The plots on the left side correspond to the electron in a non-silicon fiducial volume, and the plots on the right side correspond to the electron in the silicon fiducial volume. The excess of events at low $\eta$ for events in the silicon fiducial volume is the background from QCD $\gamma+$ jet events. (b) The same plots for the QCD $\gamma+j$ et MC. 
We are only concerned with isolated electron-like jet objects which fake an electron. Therefore, the final state electron like object is required to satisfy the isolation requirement for a signal electron. To satisfy this condition, $E_{i s o}(j e t)$ should be less than the minimum of the $E_{i s o}$ requirement. The equations below relate the fragmentation energy fraction(z), and the isolation energy requirement. The isolation energy requirement sets a limit on $\mathrm{z}$ in each topology.

$$
\begin{gathered}
E_{i s o}(\text { jet })=E_{T}(\text { parton })-E_{T}(e)=(1-z) \times E_{T}(\text { parton }) \\
(1-z) \times E_{T}(\text { parton })=\frac{1-z}{z} \times E_{T}(e) \\
\frac{1-z}{z} \times E_{T}(e)\left(=E_{\text {iso }}(\text { jet })\right)<E_{\text {iso }}(\text { min })(=4 G e V) \\
z>\frac{E_{T}(e)}{E_{\text {iso }}(\min )+E_{T}(e)}
\end{gathered}
$$

The fragmentation fraction, $z$ is limited by the two requirements specified in equation 4.1 and 4.5. Equation 4.2 is an assumption used to simplify the analysis. Figure 4.12 shows the region of phase space in $z$ for which both equations are satisfied. Most of partons in the selection are expected to be light quarks.

Heavy quarks such as the b-quark or c-quark are massive. Therefore, a b-hadron or c-hadron fragmenting from heavy quarks carries most of the quark's energy, which results in a probability distribution for $z$ which peaks close to 1.0. However, the probability for the fragmentation of light quarks is distributed randomly over all the $z$ range because the mass of the quarks is very small. Therefore, we assume that the probability function for $z$ is flat at fixed $E_{T}$. Every event is weighted by the phase space of $z$ and $E_{T}$ of the parton. The weighting factor for the event is

$$
\left(\frac{1-z_{\min }}{1}\right)
$$




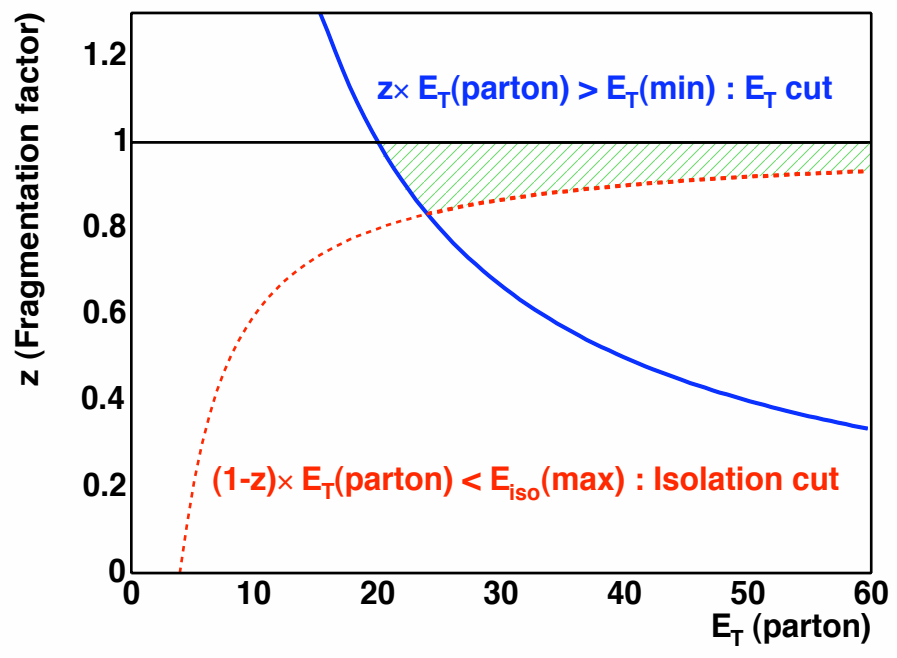

Figure 4.12: The phase-space for which the fragmentation factor $(\mathrm{z})$ is satisfied by the requirements of equation 4.1 and 4.5. The blue solid lines represents $E_{T}$ cut boundary, and the red dashed line represents the limit of the Isolation $E$ requirement. The green area is the region which is satisfied by both the $E_{T}$ and Isolation E requirements. In this plot $E_{T}>20 \mathrm{GeV}$ is required.

where $z_{\min }$ is the minimum value of $z$ at a certain $E_{T}$ (parton). Here, $\left(1-z_{\min }\right)$ is the probability to satisfy the requirement of $E_{T}$ and $E_{i s o}$ for a given $E_{T}$ (parton). The $z$ value used is randomly selected in the valid $z$ range $\left(z_{\min }\right.$ to 1$)$ assuming that the probability density of $z$ is flat.

The mass distribution of QCD $\gamma+$ jet events weighted by the corresponding $z$ factor is shown in Figure 4.13. In the low dielectron mass region, the $E_{T}$ threshold affects the shape of the mass distribution. It is difficult to get the correct shape without a full detector simulation. Since we are not doing a full simulation, we get the shape of the mass distribution a low mass from the data by subtracting the QCD dijet background fraction as estimated using the isolation extrapolation method. 
We also subtract the small amount of background from other electroweak processes, as estimated by the electroweak MC simulation samples. At high mass above the threshold, we use the $\gamma+$ jet simulated data. The slope there is exponential, so we fit it to an exponential function and use their fitted shape. The mass distribution (from the data) at low mass and the mass distribution form the QCD $\gamma+$ jet Monte Carlo at high mass are forced to be continuous at a dielectron mass of $70 \mathrm{GeV} / \mathrm{c}^{2}$.

\subsubsection{Fitting the Z/Drell-Yan dielectron Mass Distribution}

We fit the Z/Drell-Yan dielectron mass distribution to a sum of signal and background templates. Both electrons in the $\mathrm{Z}(\mathrm{CC})$ sample are required to have a track. The two-track category in $\mathrm{Z}(\mathrm{CP})$ and $\mathrm{Z}(\mathrm{PP})$ events are associated with tracks on both legs. Therefore, $\mathrm{Z}$ event samples for which both electrons have an associated track can only include the QCD dijet and electroweak backgrounds.

In other hand, the 1-track $\mathrm{Z}(\mathrm{CP})$ and $\mathrm{Z}(\mathrm{PP})$ event samples also include an additional background component originating QCD $\gamma+$ jet. In this study, we define the background derived from the background sample which is selected by the antielectron cut on one leg as the QCD "dijet" background, because it is dominated by QCD dijets (although it contains a small QCD $\gamma+$ jet component from photon conversions). The remaining $\mathrm{QCD} \gamma+$ jet background in the data is defined as the QCD " $\gamma+$ jet" background.

To obtain the level of the QCD $\gamma+$ jet background we fit the dielectron mass distribution to the sum of the contributions from Z/Drell-Yan signal events, the backgrounds from QCD dijet events, the very small contribution from all other electroweak backgrounds, and the mass distribution of the background from the QCD $\gamma+$ jet process. The small contribution of all other electroweak backgrounds is estimated from 


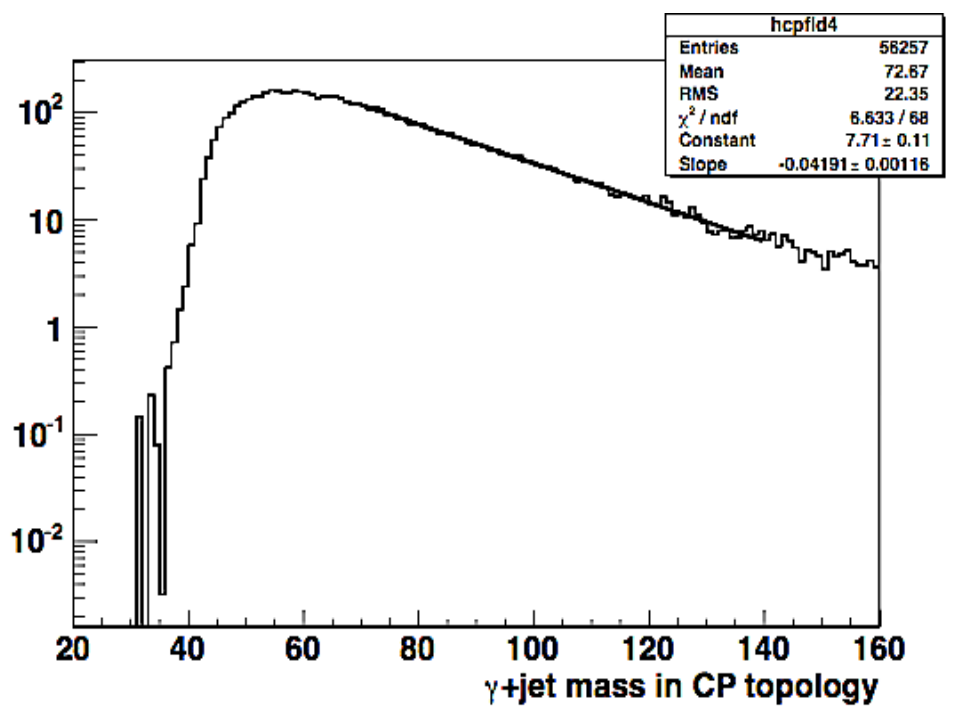

(a) CP topology

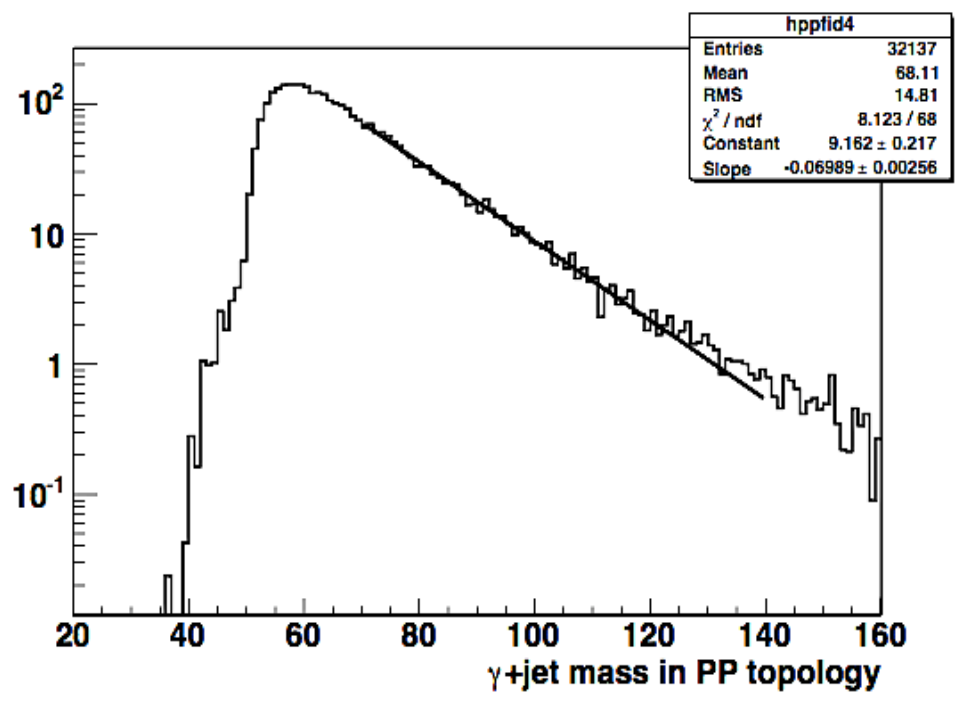

(b) PP topology

Figure 4.13: The mass distribution of QCD $\gamma+$ jet events in the MC simulation sample 
the electroweak MC simulation samples, and is held fixed in the fit for all event categories and topologies. The level of the QCD "dijet" background as estimated from the isolation extrapolation method is also held fixed in the fits.

For 1-track $\mathrm{Z}(\mathrm{CP})$ events, the background sample for the QCD "dijet" background is obtained by applying the anti-electron cut on the central leg (the tagged leg) and the plug electron is used as the target leg. The background fraction estimated by the plug leg isolation energy fit is used as the fixed level QCD "dijet" background fraction for $\mathrm{Z}(\mathrm{CP})$ events.

For 1-track $\mathrm{Z}(\mathrm{PP})$ events in the silicon fiducial region, we apply the anti-electron cut on the plug leg with a track (the tagged leg) and the background sample is obtained from the non-track electron as a target leg in the IsolationE fit. The background fraction as estimated by the isolation energy fit to the no-track electron leg is used as the fixed QCD "dijet" fraction for Z(PP) events.

As a check, we redo the fit to the dielectron Z/Drell-Yan mass distribution and also allow both the fraction of the QCD "dijet" and " $\gamma+j e t$ " backgrounds to float in the fit. We use the result of the second fit to estimate the systematic uncertainty. For $\mathrm{Z}(\mathrm{CC})$ events, 2 track events in $\mathrm{Z}(\mathrm{CP})$ and $\mathrm{Z}(\mathrm{PP})$, the overall $\mathrm{QCD}$ background level is very small.

Table 4.5 summarizes the background fractions which are estimated from the fits to the dielectron Z/Drell-Yan mass distribution. Figure 4.14, 4.15, and 4.16 show the background obtained from the Z/Drell-Yan mass distribution fits. As seen in Table 4.5, a $\chi^{2}$ comparison between the two fits shows that the fit results when we allow all parameters to float has a slightly (but not significantly) better $\chi^{2}$.

The fit to the $\mathrm{Z}(\mathrm{CP})$ topology with 2-track events is slightly lower than the data 
in low mass range. Therefore, a residual QCD $\gamma+$ jet component from conversion is included in the fit to check if any QCD $\gamma+$ jet background contributes to this category (the photon conversion rate is $5 \%$ ). The low mass region is better fit if we include the QCD $\gamma+$ jet background. However, the total background rate extracted from both fits is consistent within the statistical uncertainty. The background rate when the QCD $\gamma+j e t$ contribution is included in the fit is $0.0064 \pm 0.0013$. The background rate extracted without including the QCD $\gamma+$ jet contribution is $0.0077 \pm 0.0020$. The plot in Fig 4.15 (a) shows the fit result including the QCD $\gamma+j$ et contribution.

For the nominal determination of the QCD total background rate, the QCD "dijet" background rate is estimated using the isolation fit method, and the QCD " $\gamma+j e t$ " background rate is estimated from the fit to the $\mathrm{Z}$ mass/Drell-Yan dielectron mass distribution. (The level of QCD "dijet" background is fixed in the Z/Drell-Yan mass fit). The isolation method and mass method have different mixes of dijet and $\gamma+j e t$ backgrounds. We need them both. The $\gamma+$ jet background has larger systematic errors as it is not from a full simulation.

The difference in the overall QCD "dijet" and QCD " $\gamma+$ jet" background rates estimated from the Z/Drell-Yan mass fit with and without letting the level of the QCD "dijet" background float is used as one of the contributions to the systematic uncertainty in the total QCD background.

\subsection{Systematic Uncertainty}

One source of the systematic uncertainty in the estimate of the overall QCD background originates from the fit method itself, namely the isolation extrapolation fit 
(a) Background Fraction

\begin{tabular}{|c|l|c|c|c|}
\hline Topology & Category & & Rate(fix dijet) & Rate(free dijet) \\
\hline \hline $\mathrm{Z}(\mathrm{CC})$ & & & & $0.0012 \pm 0.0014$ \\
\hline \hline $\mathrm{Z}(\mathrm{CP})$ & 2 track & & & $0.0077 \pm 0.0020$ \\
\hline & 1 track + fid & dijet & $0.0558 \pm 0.0005$ & $0.0589 \pm 0.0060$ \\
& & $\gamma+j e t$ & $0.0396 \pm 0.0021$ & $0.0387 \pm 0.0027$ \\
& & QCD & $0.0954 \pm 0.0022$ & $0.0976 \pm 0.0065$ \\
\hline & 1 track + nfid & dijet & $0.0058 \pm 0.0001$ & $0.0148 \pm 0.0034$ \\
& & $\gamma+j e t$ & $0.0071 \pm 0.0017$ & $0.0050 \pm 0.0018$ \\
& & QCD & $0.0129 \pm 0.0017$ & $0.0198 \pm 0.0039$ \\
\hline \hline Z(PP) & 2 track & & & $0.0044 \pm 0.0023$ \\
\hline & 1 track + fid & dijet & $0.1002 \pm 0.0014$ & $0.1178 \pm 0.0145$ \\
& & $\gamma+j e t$ & $0.0578 \pm 0.0045$ & $0.0508 \pm 0.0073$ \\
& & QCD & $0.1580 \pm 0.0047$ & $0.1686 \pm 0.0163$ \\
\hline & 1 track + nfid & dijet & $0.0143 \pm 0.0002$ & $0.0097 \pm 0.0051$ \\
& & $\gamma+j e t$ & $0.0000 \pm 0.0010$ & $0.0000 \pm 0.0038$ \\
& & QCD & $0.0143 \pm 0.0011$ & $0.0097 \pm 0.0063$ \\
\hline
\end{tabular}

(b) $\chi^{2}$ comparison from the fitter

\begin{tabular}{|c|l|c|c|}
\hline Topology & Category & $\chi^{2} / \mathrm{NDF}$ (fix dijet) & $\chi^{2} / \mathrm{NDF}$ (free dijet) \\
\hline $\mathrm{Z}(\mathrm{CC})$ & & & $127.2 / 38$ \\
\hline $\mathrm{Z}(\mathrm{CP})$ & 2 track & & $23.51 / 34$ \\
& 1 track + fid & $67.61 / 43$ & $67.35 / 42$ \\
& 1 track + nfid & $52.32 / 38$ & $45.32 / 37$ \\
\hline $\mathrm{Z}(\mathrm{PP})$ & 2 track & & $69.24 / 38$ \\
& 1 track + fid & $35.76 / 38$ & $34.27 / 37$ \\
& 1 track + nfid & $46.82 / 35$ & $45.99 / 34$ \\
\hline
\end{tabular}

Table 4.5: The background rate measured by Z/Drell-Yan mass fit. Here, QCD includes the overall background for both QCD "dijet" and QCD " $\gamma+j e t$ " backgrounds. The "fid" means silicon fiducial region and the "nfid" means non-silicon fiducial region. 


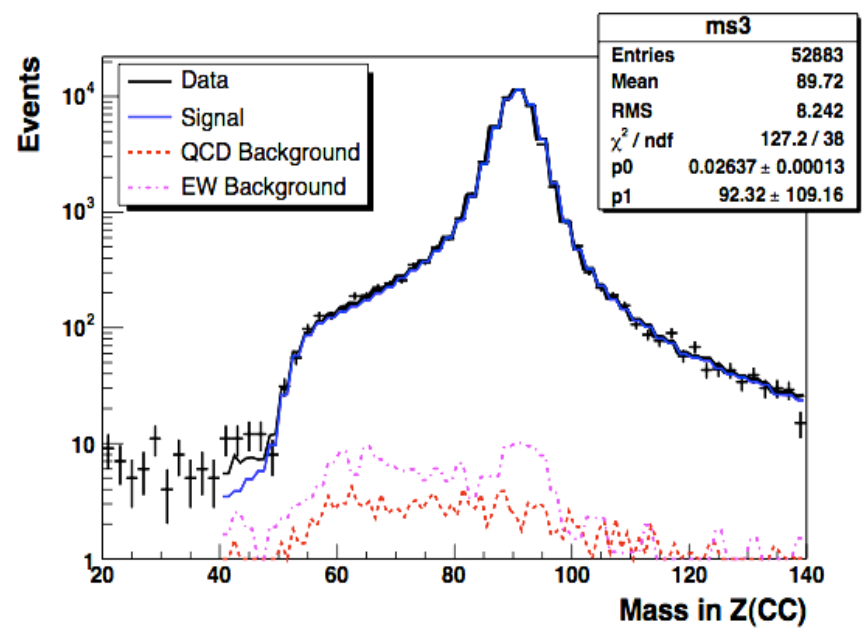

Figure 4.14: The Z/Drell-Yan mass fit result for Z(CC) events.

method and the Z /Drell-Yan mass fit method. To measure the systematic uncertainty in the method, we measure the background fraction by allowing all of background components to float in the fit to the Z/Drell-Yan dielectron mass distribution. The Z/Drell-Yan mass fit prefers a certain of amount of QCD dijet and QCD $\gamma+$ jet background fractions. As mentioned earlier, the difference between the fit with all parameters allowed to float, and the standard method (using a fixed QCD "dijet" fraction as measured by the Isolation extrapolation method) is considered as one source of systematic uncertainty in the determination of the total QCD background.

Another source of systematic uncertainty originates from the uncertainty in the shape of the QCD $\gamma+$ jet mass distribution. The QCD $\gamma+$ jet MC sample is not a full $\mathrm{QCD} /$ parton fragmentation simulation with detector simulation. To get a realistic QCD $\gamma+$ jet mass distribution, we need to know the fragmentation function to apply to the $E_{T}$ of the parton. The range of fragmentation fraction $(z)$ is determined by the $E_{T}$ and $E_{i s o}$ requirements. In this case, the $E_{i s o}$ requirement restricts the $z$ region 


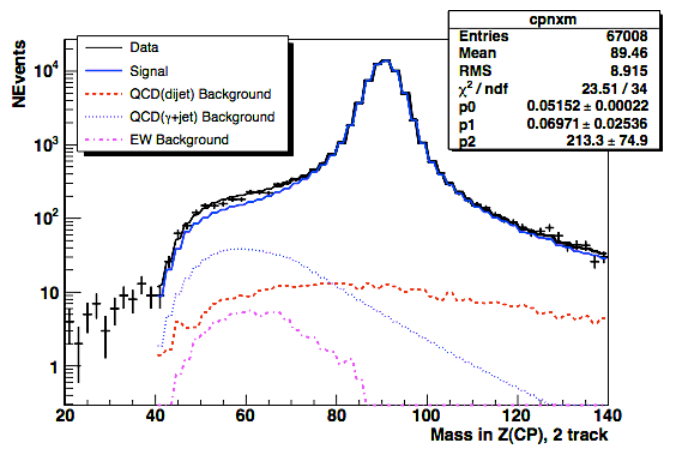

(a) 2 track
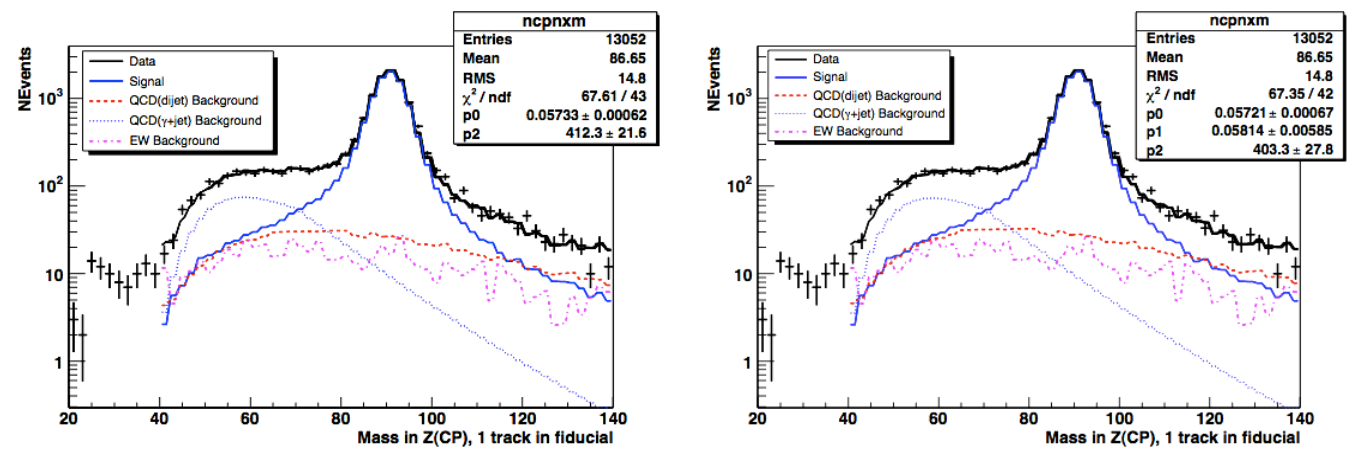

(b) 1 track and no-track leg in silicon fiducial volume (Left: fix dijet rate, Right: free dijet rate)
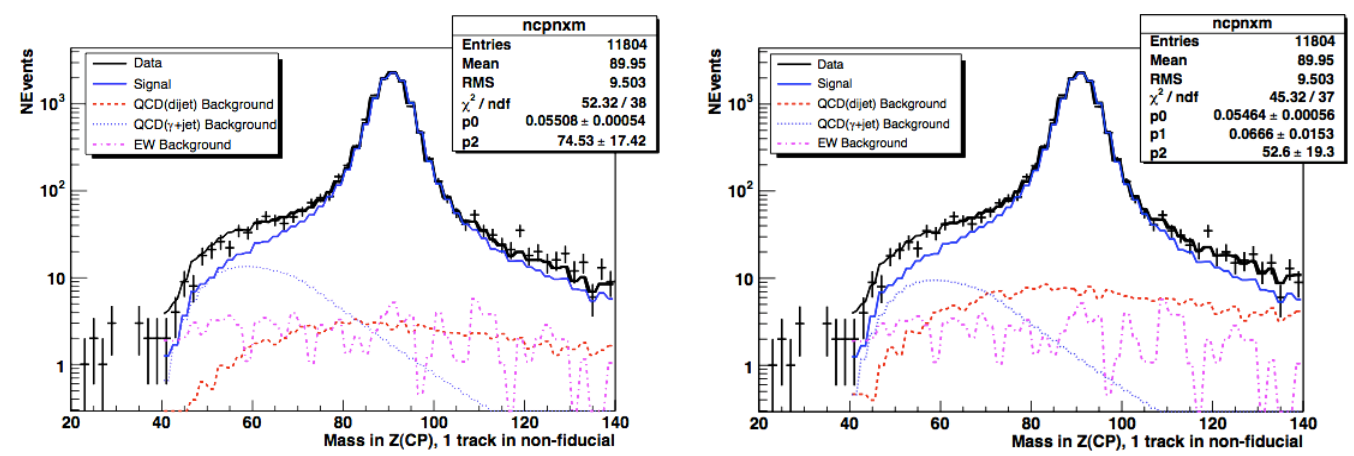

(c) 1 track and no-track leg in non-silicon fiducial volume (Left: fix dijet rate, Right: free dijet rate)

Figure 4.15: The Z/Drell-YAn mass fit result for Z(CP)events . 


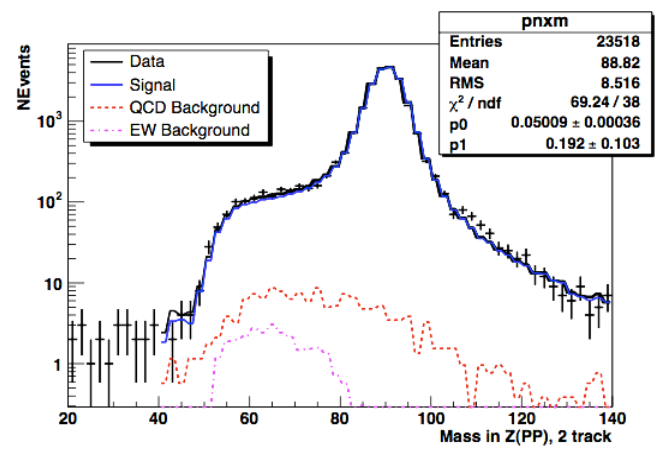

(a) 2 track
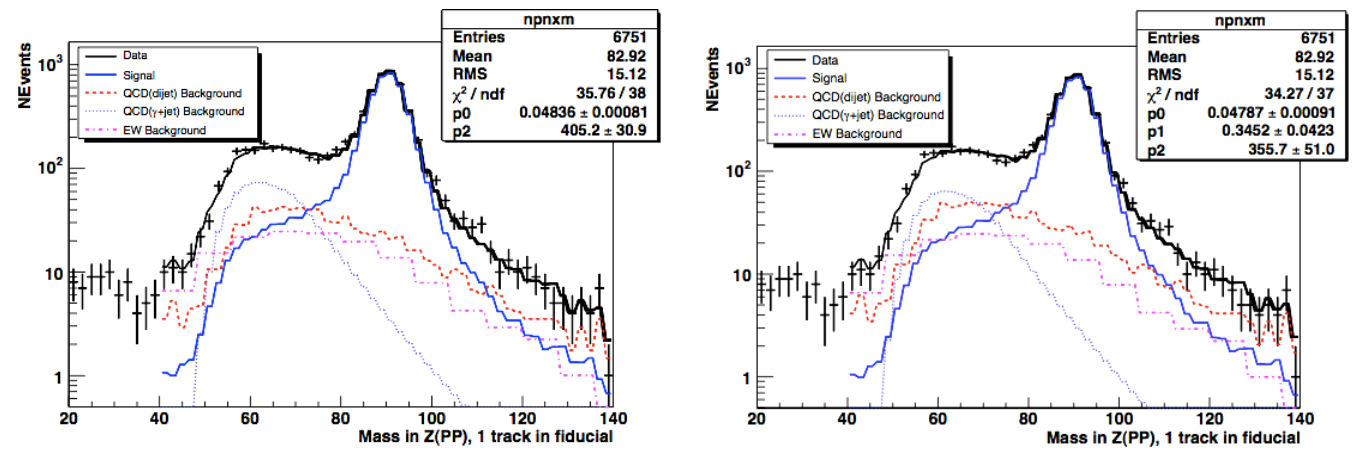

(b) 1 track and no-track leg in silicon fiducial volume (Left: fix dijet rate, Right: free dijet rate)
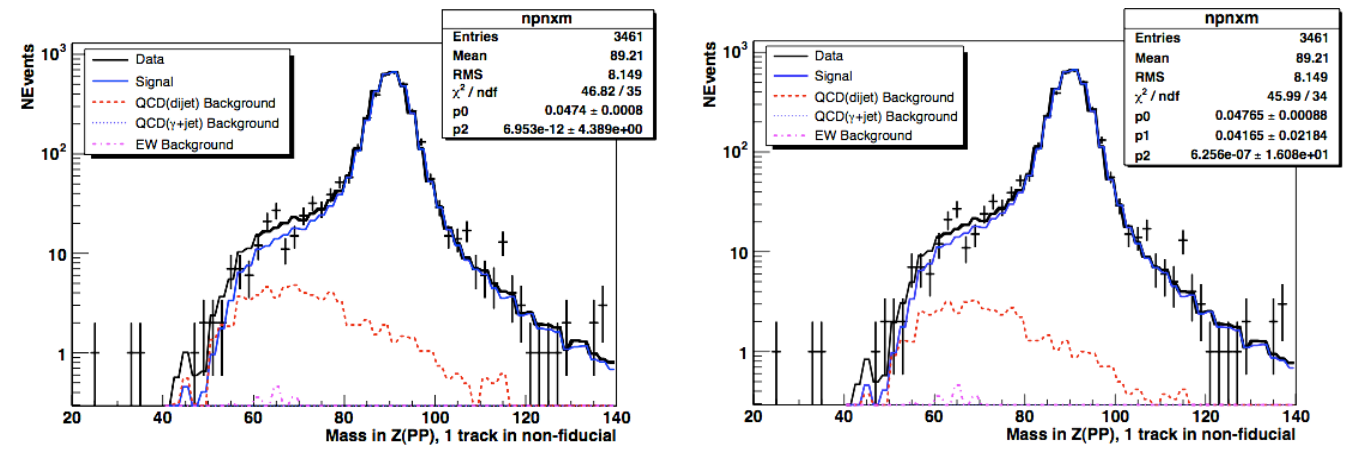

(c) 1 track and no-track leg in non-silicon fiducial volume (Left: fix dijet rate, Right: free dijet rate)

Figure 4.16: The Z/Drell-Yan mass fit result for Z(PP) events. 
more than the $E_{T}$ requirement.

In estimating the background from the QCD $\gamma+j e t$ process, we assume that the energy response of the calorimeter $(\mathrm{E} / \mathrm{P})$ is 1.0 . The $E_{\text {iso }}$ variable is sensitive to $E / P$ which affects the QCD $\gamma+$ jet mass distribution. We assume that $E / P=1.0$. Here, $E / P$ is defined as the energy response in the calorimeter for a particle, divided by the momentum of the particle. To get an estimate of the error, the QCD $\gamma+j$ et mass distribution is estimated using different $E / P$ ( $E / P$ is varied from 0.7 to 1.0$)$ Figure 4.17 shows the $z$ range changes with different $E / P$ assumptions. $(E / P=X$ corresponds to $\left.E_{i s o} / X\right)$. Table 4.6 summarize the slope of the shape of the QCD $\gamma+j e t$ mass distribution for different values of $E / P$. We fit the shape of the dielectron mass distribution of the QCD $\gamma+$ jet background with one exponential function. The slope falls faster when we release the $E_{i s o}$ constraint, because the contribution from the low $E_{T}$ (parton) region becomes larger. The systematic uncertainty in the QCD $\gamma+j$ et background is estimated from the difference in the shape of the mass distributions with $E_{i s o} / 0.7$ and $E_{i s o} / 1.0$.

Table 4.8 summarizes the systematic uncertainty in the estimated background rate for 1- track events. For the systematic uncertainty we use the background rate from the Z/Drell-Yan mass fit, letting the QCD dijet and QCD $\gamma+$ jet parameters float, and using the QCD $\gamma+$ jet shape for the mass of the two electron-like objects obtained with $E_{i s o} / 0.7$ for phase-space for the fragmentation fraction (instead of $E_{i s o} / 1.0$ ). The difference in the background rates assuming $E / P=0.7$ versus $E / P=1.0$ leads to the largest systematic uncertainty. We take the deviation between the standard method and the background rate as measured with the Z/Drell-Yan fit with the mass distribution for QCD $\gamma+j e t$ with $E / P=0.7$ as the systematic uncertainty. 


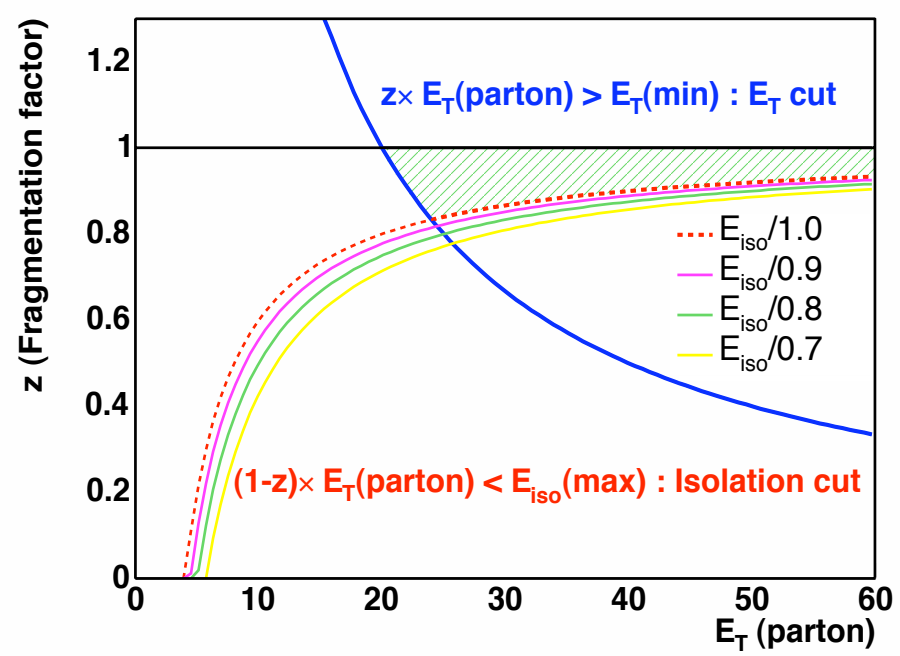

Figure 4.17: The phase-space for which the fragmentation factor, $\mathrm{z}$ is satisfied by the requirements of equation 4.1 and 4.5. The blue solid line represent the $E_{T}$ cut boundary and the red dashed line represents the limit from the isolation E requirement. The green area is the region which is satisfied by both $E_{T}$ and isolation $\mathrm{E}$ requirements. In this plot, $E_{T}>20 \mathrm{GeV}$ is required. Here, $E / P=X$ corresponds to $E_{i s o} / X$. Different $E / P$ assumptions on the energy response of the calorimeter lead to a changes in the allowed $z$ region which affects the QCD $\gamma+$ jet mass distribution. 
(a) $\gamma+$ jet mass in $\mathrm{Z}(\mathrm{CP})$ topology

\begin{tabular}{|c|c|}
\hline$E_{\text {iso }} / x$ & Slope of $\gamma+$ jet mass \\
\hline$E_{\text {iso }} / 1.0$ & $-0.0419 \pm 0.0012$ \\
$E_{\text {iso }} / 0.9$ & $-0.0436 \pm 0.0012$ \\
$E_{\text {iso }} / 0.8$ & $-0.0453 \pm 0.0013$ \\
$E_{\text {iso }} / 0.7$ & $-0.0477 \pm 0.0014$ \\
\hline
\end{tabular}

(b) $\gamma+$ jet mass in $\mathrm{Z}(\mathrm{PP})$ topology

\begin{tabular}{|c|c|}
\hline$E_{\text {iso }} / x$ & Slope of $\gamma+$ jet mass \\
\hline$E_{\text {iso }} / 1.0$ & $-0.0699 \pm 0.0026$ \\
$E_{\text {iso }} / 0.9$ & $-0.0722 \pm 0.0029$ \\
$E_{\text {iso }} / 0.8$ & $-0.0738 \pm 0.0033$ \\
$E_{\text {iso }} / 0.7$ & $-0.0775 \pm 0.0038$ \\
\hline
\end{tabular}

Table 4.6: The slope of the mass distribution of the two electron-like objects origination from QCD $\gamma+$ jet for different range of the fragmentation factor, $z$. Here $E_{\text {iso }} / X$ corresponds to $E / P=X$. The difference between $E / P=0.7$ and $E / P=1.0$ is used to estimate the systematic uncertainty originating from modeling the shape of the QCD $\gamma+$ jet distribution. 


\begin{tabular}{|c|c|c|}
\hline Topology & Isolation Method & Z Mass Method \\
\hline $\mathrm{Z}(\mathrm{CC})$ & $0.0024 \pm 0.0003$ & $0.0012 \pm 0.0014$ \\
$\mathrm{Z}(\mathrm{CP})$ & $0.0022 \pm 0.0003$ & $0.0077 \pm 0.0020$ \\
$\mathrm{Z}(\mathrm{PP})$ & $0.0067 \pm 0.0012$ & $0.0044 \pm 0.0023$ \\
\hline
\end{tabular}

Table 4.7: The background fraction in 2-track case. Only statistical uncertainty are shown. The background rate estimated by the isolation extrapolation method agrees with the background rate from the $\mathrm{Z}$ mass/Drell Yan fit within the statistical uncertainties for both $\mathrm{Z}(\mathrm{CC})$ and $\mathrm{Z}(\mathrm{CP})$. For 2-track events in all topologies, the background fraction is very small (below $1 \%$ ).

For $\mathrm{Z}(\mathrm{CC})$ and $\mathrm{Z}(\mathrm{PP})$ two track events (for which the background is very small) the difference between the Isolation extrapolation method and the fit to the Z/DrellYan dielectron mass distribution is taken as the systematic uncertainty (because very few QCD $\gamma+$ jet two track events are expected from photon conversions). For two track events, an additional systematic uncertainty is not assigned because the statistical uncertainty from the fit to the Z/Drell-Yan dielectron mass distribution covers difference in the background rates between the Isolation extrapolation method and the rate from the fit to the Z/Drell-Yan dielectron mass distribution. For 2-track events in all topologies, the background fraction is very small (below 0.007). Table 4.7 summarizes the background fraction in 2-track events.

The total systematic uncertainty is estimated to be $27 \%$ of the background rate in $\mathrm{Z}(\mathrm{CP})$, and $6 \%$ of the background rate for $\mathrm{Z}(\mathrm{PP})$. Figure 4.18 shows the uncertainties in the background in each topology. 
(a) Background Fraction

\begin{tabular}{|c|c|c|c|c|}
\hline Topology & Category & & Rate(free dijet) & Rate(free dijet $+E / P=0.7)$ \\
\hline \hline $\mathrm{Z}(\mathrm{CP})$ & 1 track + fid & dijet & $0.0589 \pm 0.0060$ & $0.0602 \pm 0.0059$ \\
& & $\gamma+j$ et & $0.0387 \pm 0.0027$ & $0.0367 \pm 0.0026$ \\
& & QCD & $0.0976 \pm 0.0065$ & $0.0969 \pm 0.0064$ \\
\hline & 1 track + nfid & dijet & $0.0148 \pm 0.0034$ & $0.0149 \pm 0.0034$ \\
& & $\gamma+j$ et & $0.0050 \pm 0.0018$ & $0.0048 \pm 0.0018$ \\
& & QCD & $0.0198 \pm 0.0039$ & $0.0197 \pm 0.0038$ \\
\hline \hline $\mathrm{Z}(\mathrm{PP})$ & 1 track + fid & dijet & $0.1178 \pm 0.0145$ & $0.1198 \pm 0.0143$ \\
& & $\gamma+j e t$ & $0.0508 \pm 0.0073$ & $0.0484 \pm 0.0070$ \\
& & QCD & $0.1686 \pm 0.0163$ & $0.1682 \pm 0.0159$ \\
\hline & 1 track + nfid & dijet & $0.0097 \pm 0.0051$ & $0.0097 \pm 0.0051$ \\
& & $\gamma+j e t$ & $0.0000 \pm 0.0038$ & $0.0000 \pm 0.0035$ \\
& & QCD & $0.0097 \pm 0.0063$ & $0.0097 \pm 0.0062$ \\
\hline
\end{tabular}

(b) $\chi^{2}$ comparison from the fitter

\begin{tabular}{|c|l|c|c|}
\hline Topology & Category & $\chi^{2} / \mathrm{NDF}($ free dijet) & $\chi^{2} / \mathrm{NDF}$ (free dijet $+E / P=0.7$ ) \\
\hline $\mathrm{Z}(\mathrm{CP})$ & 1 track + fid & $67.35 / 42$ & $67.93 / 42$ \\
& 1 track + nfid & $45.32 / 37$ & $45.37 / 37$ \\
\hline $\mathrm{Z}(\mathrm{PP})$ & 1 track + fid & $34.27 / 37$ & $34.57 / 37$ \\
& 1 track + nfid & $45.99 / 34$ & $45.99 / 34$ \\
\hline
\end{tabular}

Table 4.8: The background rate measured using the Z/Drell-Yan mass fit. QCD means the overall backgrounds of QCD "dijet" and QCD " $\gamma+j e t$ " processes. The rate with "free dijet" means that the normalization parameters for the QCD "dijet" and QCD " $\gamma+j e t$ " are free to float. The $E_{\text {iso }} / 0.7$ is the $E_{\text {iso }}$ requirement for determining the fragmentation fraction factor. The "fid" means silicon fiducial region and the "nfid" means non-silicon fiducial region. 


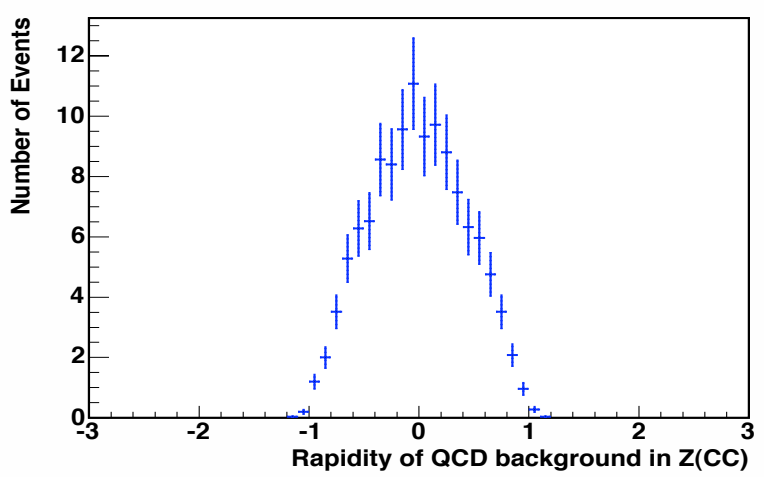

(a) QCD background in $\mathrm{Z}(\mathrm{CC})$

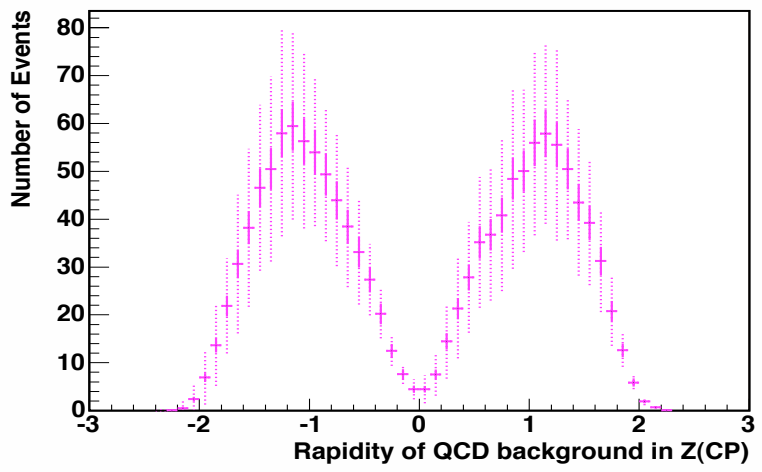

(b) QCD background in Z(CP)

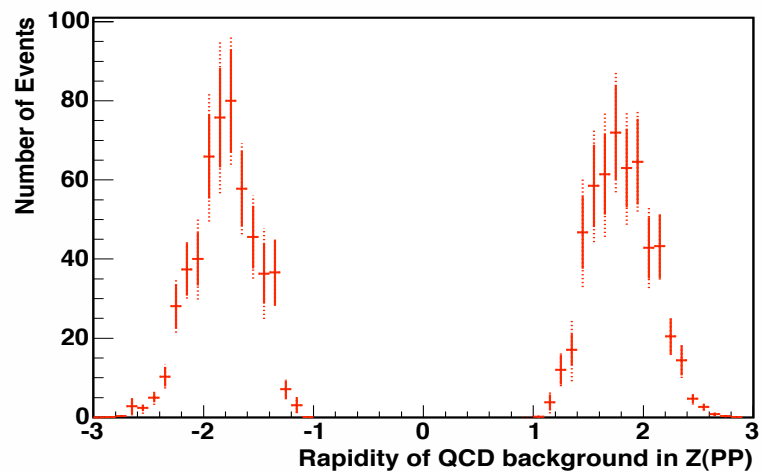

(c) QCD background in Z(PP)

Figure 4.18: The rapidity distribution for the total QCD background. The solid line is statistical uncertainty only. The dashed line is the systematical uncertainty. 


\subsection{Total Background Rate}

In summary, the QCD background is measured using the Isolation extrapolation fit in combination with the fit to the Z/Drell-Yan dilepton mass distribution. The QCD "dijet" background rate is measured by the isolation extrapolation method. The QCD " $\gamma+$ jet" background rate is measured using the fit to the Z/Drell-Yan mass distribution which includes a fixed "dijet" background fraction (as estimated with the Isolation extrapolation method). The total background fraction is $0.0024 \pm$ 0.0003(stat.) for Z(CC) events, $0.0155 \pm 0.0012$ (stat.) \pm 0.0042 (sys.) for $\mathrm{Z}(\mathrm{CP})$ events, and $0.0340 \pm 0.0072$ (stat.) \pm 0.0021 (sys.) for $\mathrm{Z}(\mathrm{PP})$ events. Figure 4.19 shows the rapidity distribution of combined QCD background in the sample.

\subsubsection{Electroweak Background}

Electroweak processes associated with the production of pair of W's, a Z boson or a pair of $\tau$ leptons can contribute to the background in this measurement.

These include:

- Di-boson processes (WW and WZ pairs) :

Events for which both W's decay to electrons, or the Z decays to electrons may end up in the dielectron signal region.

- The $W \rightarrow e \nu+$ jets $/ \gamma$ process : It also contributes if the jet or $\gamma$ object are misidentified as an electron. A misidentified jet or $\gamma$ in conjunction with an electron originating from the decay of the $\mathrm{W}$ boson may end up in the dielectron sample. The misidentification fraction for jets $/ \gamma$ is in general not large. However, the plug electron in the $\mathrm{Z}(\mathrm{CP})$ and $\mathrm{Z}(\mathrm{PP})$ samples is not required to 


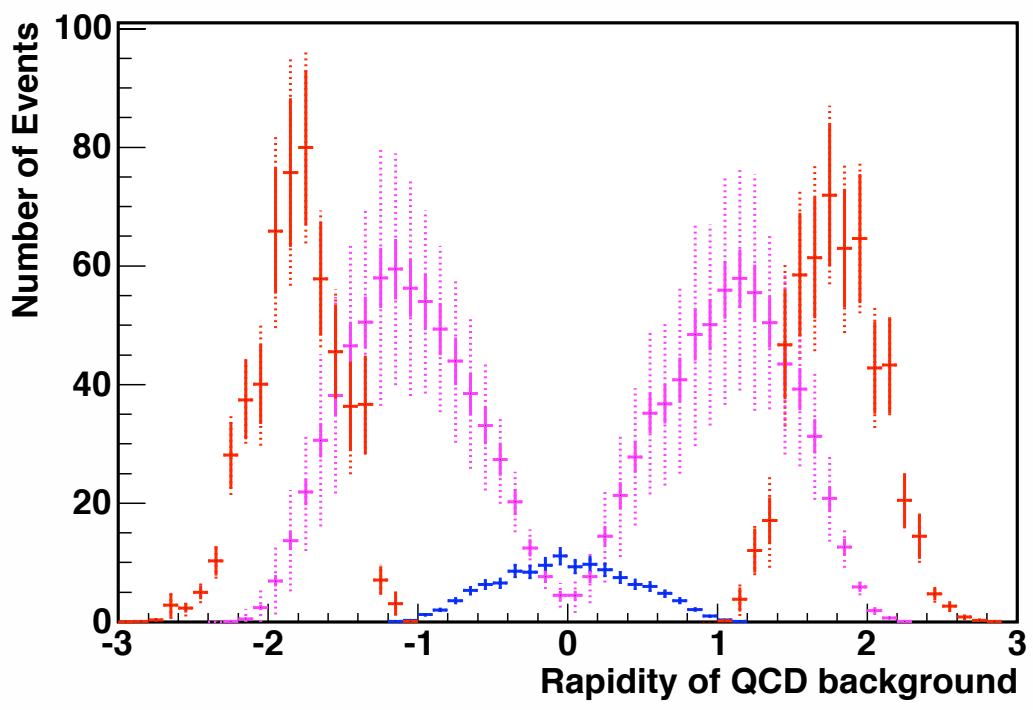

Figure 4.19: The rapidity distribution of the QCD background in each event topology (CC, $\mathrm{CP}$ and $\mathrm{PP})$. The blue histogram is the background distribution for $\mathrm{Z}(\mathrm{CC})$ events, the magenta histogram is for $\mathrm{Z}(\mathrm{CP})$ events, and the red histogram is for $\mathrm{Z}(\mathrm{PP})$ events. The solid line is the statistical uncertainty and the dashed line is the systematical uncertainty. 
have a track. This leads to a higher level of background from this process in the $\mathrm{Z}(\mathrm{CP})$ and $\mathrm{Z}(\mathrm{PP})$ topologies.

- Background from standard QCD production of a pair of top quarks :

All top quarks decay to a $\mathrm{W}$ boson and a b-jet, and the $W \rightarrow e \nu$ decay mode results in a final state electron. Similarly, the $\bar{t}$ in the same event always decays into $\mathrm{W}$ and b-jet as well. Two electrons that may originate from decays of both W bosons contribute to the background in the dielectron sample.

- $Z \rightarrow \tau \tau$ process :

Standard QCD production of a Z/Drell Yan pairs of $\tau$ leptons with each of the $\tau$ leptons decaying into a final state electron.

The background from all electroweak processes is determined using a Monte Carlo simulation. Table 4.9 summarizes the background contributions of the electroweak processes listed above for all event topologies.

Figure 4.20 shows the individual contributions of each electroweak process to the total number of electroweak background events in the $Z / \gamma^{*}$ dielectron sample for all event topologies combined.

Figure 4.21 shows the rapidity distributions of data, QCD, and electroweak backgrounds. The background from electroweak processes is very small when compared to the data. 


\begin{tabular}{|c|c|c|c|c|c|}
\hline Process & $\sigma \cdot B r(p b)$ & Zcc & Zcp & Zpp & Total \\
\hline WW & 1.4 & $19.6 \pm 1.4$ & $22.2 \pm 1.6$ & $3.3 \pm 0.3$ & $45.5 \pm 2.1$ \\
WZ & 0.4 & $34.8 \pm 2.3$ & $44.0 \pm 3.0$ & $12.5 \pm 0.8$ & $91.3 \pm 3.9$ \\
$t \bar{t}$ inclusive & 5.5 & $9.7 \pm 0.7$ & $6.6 \pm 0.5$ & $0.4 \pm 0.1$ & $16.7 \pm 1.0$ \\
Inclusive W & 2744.0 & $28.3 \pm 5.5$ & $344.9 \pm 30.6$ & $71.0 \pm 9.4$ & $444.2 \pm 32.4$ \\
$Z \rightarrow \tau \tau$ & 238.0 & $23.8 \pm 2.1$ & $47.7 \pm 3.8$ & $26.5 \pm 2.2$ & $98.1 \pm 4.9$ \\
\hline Total & & $116.5 \pm 6.5$ & $465.4 \pm 31.0$ & $113.8 \pm 9.7$ & $695.7 \pm 33.1$ \\
\hline
\end{tabular}

Table 4.9: The number of background events originating from various electroweak processes for each event topology.

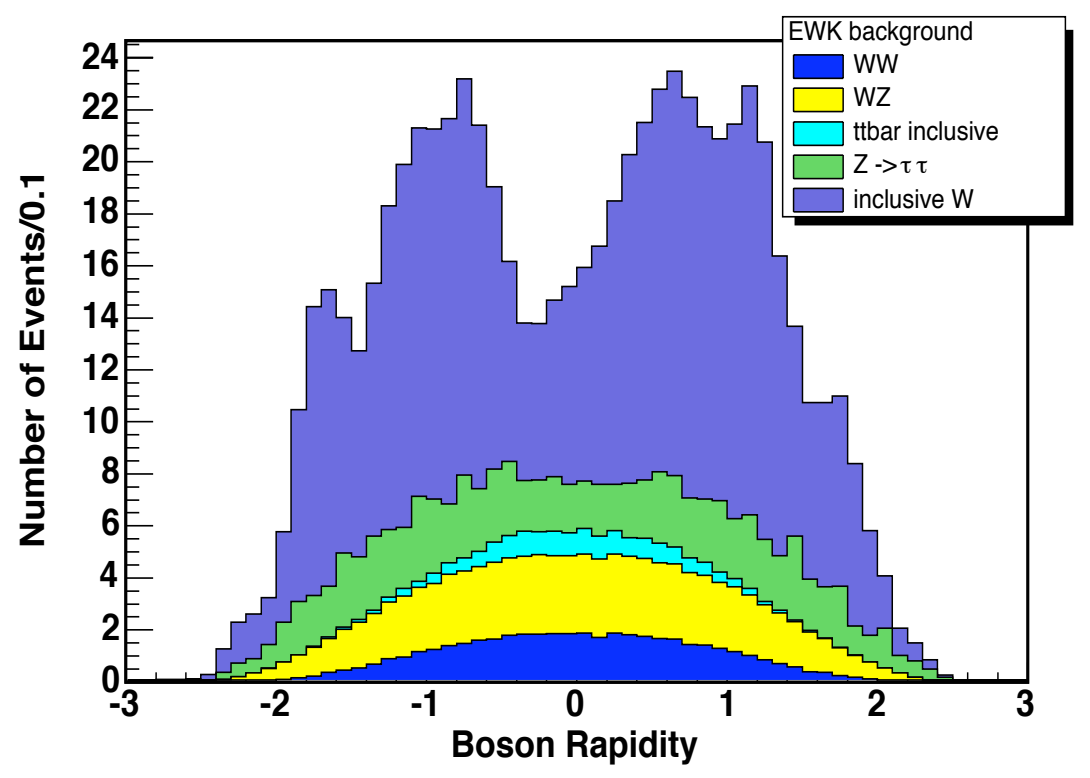

Figure 4.20: The individual contributions of each electroweak process to the total number of electroweak background events in the $Z / \gamma^{*}$ sample (for all event topologies combined). 


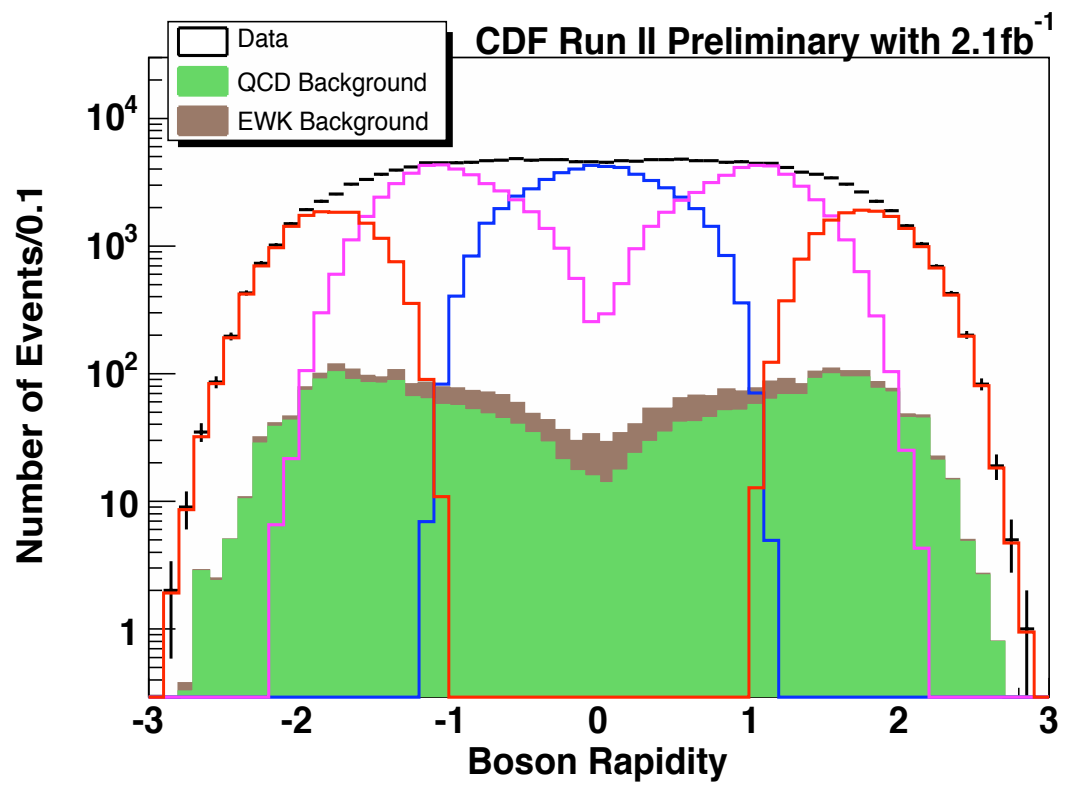

Figure 4.21: The rapidity distribution of $Z^{0} / \gamma^{*} \rightarrow e^{+} e^{-}$events. The QCD and electroweak backgrounds are also shown in the plot. The blue, magenta, and red lines are the dielectron rapidity distribution for the $\mathrm{Z}(\mathrm{CC}), \mathrm{Z}(\mathrm{CP})$, and $\mathrm{Z}(\mathrm{PP})$ topologies respectively. 


\section{Chapter 5}

\section{Acceptance and Efficiency}

\subsection{Geometric and Kinematic Acceptance}

The detector acceptance for $Z^{0} / \gamma^{*} \rightarrow e^{+} e^{-}$events is estimated using the PYTHIA Monte Carlo generator. The acceptance is defined as $N_{\text {recon }} / N_{\text {gen }}$, where $N_{\text {gen }}$ is the number of generated events and $N_{\text {recon }}$ is the number of generated events that are reconstructed events and pass geometric and kinematic selection cuts. The number of generated events $N_{\text {gen }}$ in the acceptance ratio includes only events generated with $\left|z_{\text {vertex }}^{\text {gen }}\right|<60 \mathrm{~cm}$ and with $66<M_{e e}^{\text {gen }}<116 \mathrm{GeV} / \mathrm{c}^{2}$. The number of reconstructed events in the acceptance ratio $N_{\text {recon }}$ includes only events reconstructed with $\left|z_{\text {vertex }}\right|<$ $60 \mathrm{~cm}$, with $66<M<116 \mathrm{GeV} / \mathrm{c}^{2}$, and with all other kinematic cuts described in Table 5.1. The $Z \rightarrow$ ee MC sample used in the determination of the acceptance is generated using the CTEQ5L PDFs. The CTEQ5L PDFs are PDFs in Leading Order (LO). In addition, as described below, a $K$ factor are used to account for higher order NLO QCD corrections to the LO production cross section. Finally, a model independent acceptance correction is calculated by applying weighting factors based on the measurement to the differential distribution in the model to agree with our measurements. 
The acceptance in Z/dielectron rapidity $\left(y_{Z}\right)$ depends both on the PDFs (LO, vs NLO, vs NNLO) and on higher order QCD corrections to the Z/Drell-Yan production process. The PDFs extracted in QCD LO are different from PDFs extracted for higher orders. The predicted differential cross section in $y_{z}$ for various theoretical models are compared to estimate the effect from PDFs and higher order QCD corrections. Three model predictions, CTEQ6M(NLO)[16], NLO MRST[17], and NNLO MRST[18], are investigated to estimate the effect of different PDFs and higher order QCD corrections on the acceptance. Figure 5.1 show the shape of the $d \sigma / d y_{Z}$ calculated for each of these models (normalized to be same as the cross section with CTEQ5L at $y_{Z}=0$ ). The normalized $d \sigma / d y$ theoretical prediction with CTEQ5L is different from the other predictions as shown in Figure 5.2 (a).

Plot 5.2 (b) shows how the ratio of the acceptance as calculated with $d \sigma / d y$ prediction for models with higher order QCD corrections and PDFs to the acceptance calculated with CTEQ5L in LO. Note that in addition to difference originating from the difference in the NLO and NNLO PDFs, QCD higher order corrections to the cross section also change the predicted $d \sigma / d y$ distribution. These changes are important at high values of $y_{Z}$. However, the true $d \sigma / d y$ distribution in the high $y_{Z}$ region is not known. Therefore, we use our data to measure the $d \sigma / d y$ distribution at high $y_{Z}$, and include this measurement as input in the calculation of the acceptance.

In this section, we first discuss the acceptance calculated using CTEQ5L in LO. Later on, after all background subtraction and efficiency corrections are applied to the data, a correction to the acceptance is calculated by using $d N / d y$ as measured with our data (which is an iterative process). Figure 5.3 shows the initial acceptance as a function of $y_{Z}$ as calculated using the CTEQ5L in leading order. 


\begin{tabular}{|c|c|c|}
\hline Variable & central electron & plug electron \\
\hline$E_{t}$ & $>25(C C), 20(C P) G e V$ & $>20(C P), 25(P P) G e V$ \\
Track $z_{0}$ & $\mid$ Track $z_{0} \mid<60 \mathrm{~cm}$ & \\
$P_{t}$ & $>10 \mathrm{GeV}$ & $1.2<|\eta|<2.8$ \\
\hline Fiduciality & track based fiduciality & expected $N$ of hit $\geq 3$ \\
Silicon Fiducial for Z(PP) & & reconstructed $Z$ V ertex \\
\hline
\end{tabular}

Table 5.1: Kinematic selection cuts for central and plug electrons.

The total acceptance (using CTEQ5L) for Z/Drell-Yan di-electron events is 10.70土 $0.01 \%$ for the $\mathrm{Z}(\mathrm{CC})$ topology, $23.47 \pm 0.01 \%$ for the $\mathrm{Z}(\mathrm{CP})$ topology, and $9.52 \pm 0.01 \%$ for the $\mathrm{Z}(\mathrm{PP})$ topology.

\subsection{Phoenix Tracking Efficiency}

The central tracker provides additional tracking for the central region in addition to the silicon tracker. Since the plug region does not have an additional tracker to help with electron identification, the background level in $\mathrm{Z}(\mathrm{PP})$ events is larger.

To reduce the background in the $\mathrm{Z}(\mathrm{PP})$ topology, a silicon phoenix track is required for at least one of the $\mathrm{Z}(\mathrm{PP})$ electrons. The silicon phoenix track uses a special tracking algorithm used to increase the tracking efficiency in the plug region. The phoenix track is reconstructed by using a seed track constructed from the event's $p \bar{p}$ collision vertex in z-axis, ZVertex, and the electron's position in the plug PES calorimeter. This implies that a reconstructed ZVertex is required in advance for the silicon phoenix tracking to work. Therefore, the event ZVertex is treated as an acceptance.

The silicon phoenix tracking efficiency is measured with $\mathrm{Z}(\mathrm{PP})$ events which 


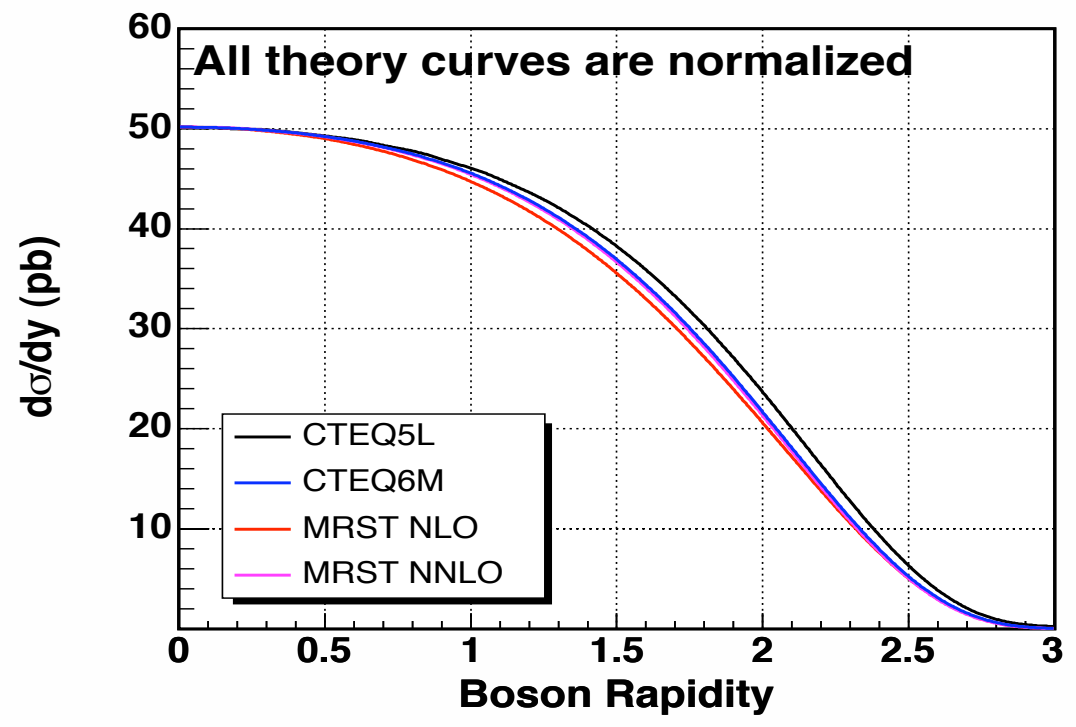

Figure 5.1: Theoretical predictions for the $d \sigma / d y$ distribution calculated for several theoretical models. All theory predictions are normalized to be same at $y_{Z}=0$ as $d \sigma / d y$ for CTEQ5L. 


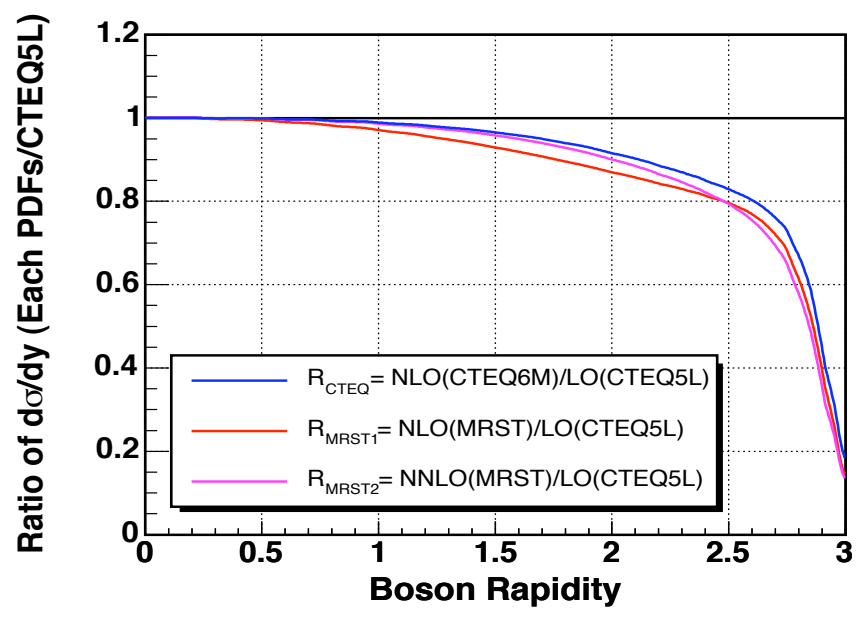

(a) The ratio of the $d \sigma / d y$ predictions for different theoretical models to CTEQ5L

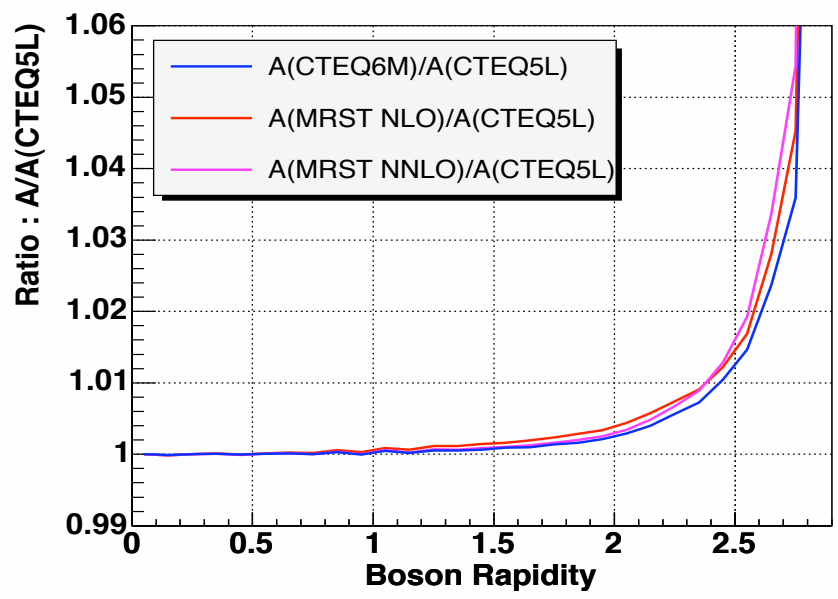

(b) The ratio of the acceptance calculated for three different theoretical models for $d \sigma / d y$ divided by the acceptance for CTEQ5L as a function of $y_{Z}$

Figure 5.2: The cross section and acceptance ratios of three different theoretical models to that with CTEQ5L. Plot (a) shows the differential cross section ratio of each theory prediction to CTEQ5L. Plot (b) shows the ratio of the acceptance calculated for three different theoretical models for $d \sigma / d y$ divided by the acceptance for CTEQ5L as a function of $y_{Z}$. The acceptance for the three theoretical models was derived from the acceptance for CTEQ5L by applying a weighting factor to CTEQ5L Monte Carlo events. The $d \sigma / d y$ ratio shown in plot (a) is used for the weighting factor as a function of $y_{Z}$. 


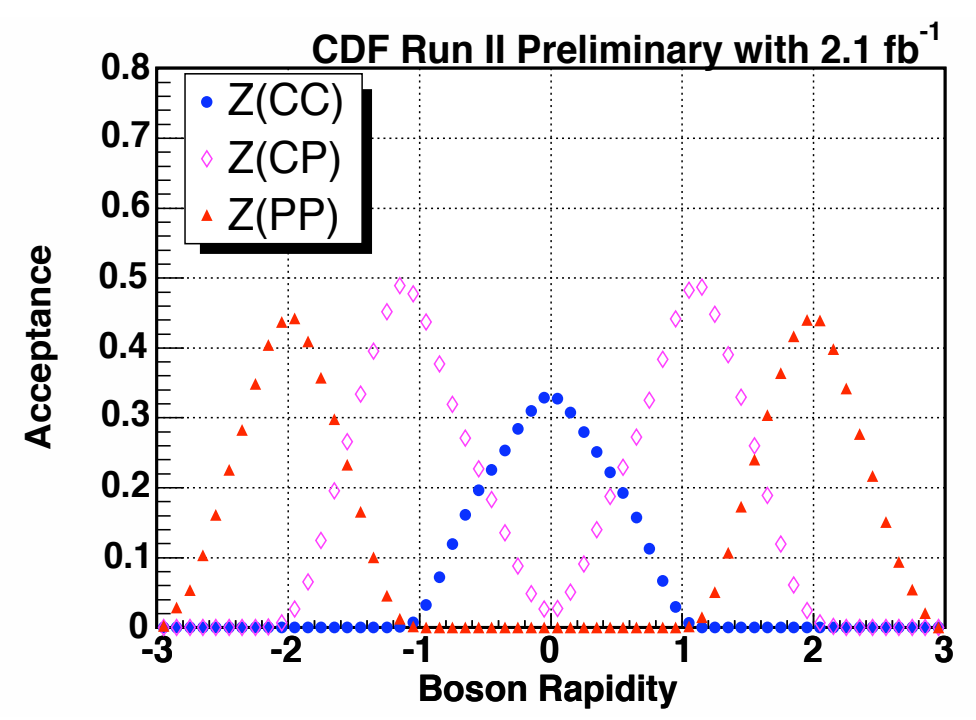

Figure 5.3: The geometric and kinematic acceptance versus $y_{Z}$ for $\mathrm{Z}(\mathrm{CC}), \mathrm{Z}(\mathrm{CP})$, and $\mathrm{Z}(\mathrm{PP})$ event topologies.

passed the track fiducial requirement. The track fiducial requirement for electrons requires that the expected number of silicon layers which the electron is expected to traverse is three or more, and that the event has a reconstructed ZVertex. The number of silicon layers which the electron is expected to traverse is calculated using the silicon detector geometry, the ZVertex, and PES position of the electron. Figure 5.4 shows the silicon detector geometry including the ISL.

To measure the efficiency of having one or more phoenix tracks in the $\mathrm{Z}(\mathrm{PP})$ sample, we need to determine the phoenix tracking efficiency for electrons, and also determine the fraction of no-track events. The efficiency and no-track event fraction are measured both in data and MC respectively, and scale factors which are the ratios of the efficiency and no-track fraction in data to $\mathrm{MC}$ are determined. The scale factors are then applied to $\mathrm{MC}$ events to tune the MC sample such that it correctly simulates 


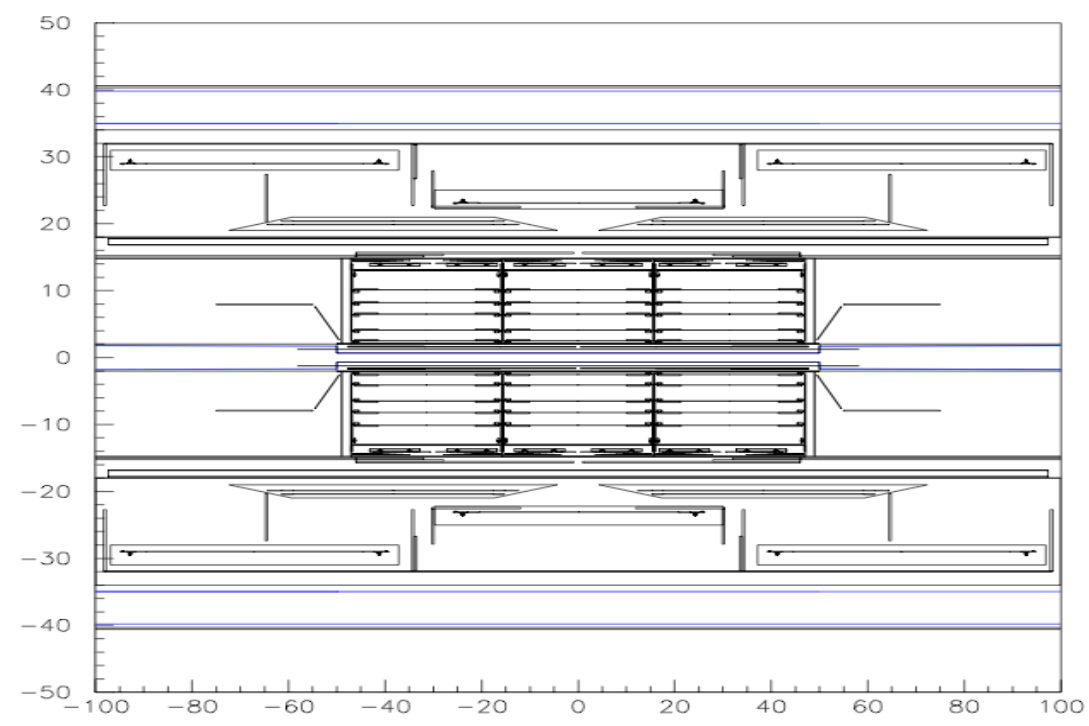

Figure 5.4: The silicon detector geometry including the ISL.

that tracking efficiency as well the efficiency for having one or more phoenix tracks as a function of $y_{Z}$. The efficiencies and fraction of no-track events are extracted from events in the $66<M_{Z}<116 \mathrm{GeV} / \mathrm{c}^{2}$ window, after applying all electron identification selection cuts (to reduce the background in the sample). The measurement of each component is described in the following subsections.

\subsubsection{The ZVertex Reconstruction Efficiency}

A reconstructed ZVertex is required as one of the kinematic selection cuts (acceptance), and are shown in Table 5.1 for $\mathrm{Z}(\mathrm{PP})$ events. However, there is a difference between the ZVertex finding efficiency in the data and in the MC simulation. To remove this discrepancy, the ZVertex reconstruction efficiency is measured in data and $\mathrm{MC}$ and a scale factor, which the efficiency ratio between data and MC (data/MC), is applied to the MC sample. This tuned MC is used to determine the acceptance for 
$\mathrm{Z}(\mathrm{PP})$ events. The ZVertex is reconstructed from tracks, so if there are insufficient tracks in an event, a ZVertex cannot be determined.

For the sample of events where no reconstructed vertex is available, the ZVertex is initially set to $z=0$ in order to reconstruct the $\mathrm{Z} /$ dilepton mass. The dilepton mass distribution is used in the background estimate for the sample. To reduce the background contamination in the sample, all electron identification requirement are applied. The background fraction in the sample is estimated by fitting the Z/dilepton mass distribution. The $Z \rightarrow e e \mathrm{MC}$ sample is used for the signal template for the dilepton mass, and the background enhanced sample used for the background template is obtained from the data by applying the anti-electron selection cuts to one of the two electrons. The shape of the dilepton mass distribution for background events with no reconstructed ZVertex is different from the background shape for events with a reconstructed ZVertex. Therefore, the background level is estimated separately for events with and without a reconstructed ZVertex separately. After background subtraction, the reconstructed ZVertex inefficiency is defined as

$$
\left(1-\epsilon_{Z v t x}\right)=\frac{\text { Number of } Z(P P) \text { events without reconstructed } Z \text { Vertex }}{\text { Number of } Z(P P) \text { events }}
$$

The ZVertex region for which there is good acceptance to particles produced in $p \bar{p}$ collisions for all subdetectors of $\mathrm{CDF}$ is for $\mid Z V$ ertex $\mid \leq 60 \mathrm{~cm}$. Therefore, we only consider events with $\mid Z V$ ertex $\mid \leq 60 \mathrm{~cm}$. If we want to find the ZVertex reconstruction efficiency for this region, we need to find a way to determine the vertex location for events which do not have a reconstructed vertex. For such events, the most likely ZVertex is determined with the Z mass constrained method. By varying ZVertex from $-150 \mathrm{~cm}$ to $150 \mathrm{~cm}$ with $0.5 \mathrm{~cm}$ steps we find the ZVertex which 
yields a dilepton invariant mass which is closest to $91 \mathrm{GeV} / \mathrm{c}^{2}$. Figure 5.5 shows the ZVertex distribution determined by the mass constrained method. The mass constrained ZVertex is used for the $\mid Z V$ ertex $\mid<60 \mathrm{~cm}$ fiducial requirement, and the mass distributions (computed using $Z$ Vertex $=0 \mathrm{~cm}$ ) are shown in the bottom plots of Figure 5.6. We use the signal + background plot on the bottom right side of Figure 5.6 to measure the fraction of $\gamma^{*} / Z$ boson events with no ZVertex. The ZVertex finding inefficiency is measured to be $\left(1-\epsilon_{Z v t x}\right)=0.0356 \pm 0.0013$ in data and $\left(1-\epsilon_{Z v t x}\right)=0.0176 \pm 0.0001$ in MC. Therefore, the scale factor for the ZVertex reconstruction efficiency is measured as:

$$
\frac{\epsilon_{Z v t x}^{D a t a}}{\epsilon_{Z v t x}^{M C}}=0.9817 \pm 0.0013
$$

The scale factor for the ZVertex finding efficiency is applied the MC sample in the determination of the acceptance for the $\mathrm{Z}(\mathrm{PP})$ event topology.

For a systematic study of the ZVertex finding efficiency, we find the dependence of the efficiency on the ZVertex and $M_{Z}$ cuts. We measure the efficiency with and without the $\mid Z V$ ertex $\mid \leq 60 \mathrm{~cm}$ requirement. We also measure the efficiency with the a background subtraction using the background dilepton mass distribution from the events with and without a reconstructed ZVertex. We also measure the efficiency with and without the $66<M_{Z}<116 \mathrm{GeV} / \mathrm{c}^{2}$ cut on the dilepton invariant mass. Table 5.2 summarizes the efficiencies and scale factors measured for each condition. The largest deviation in the measurements of the scale factor for the ZVertex finding efficiency is 0.005 . We use this value as the systematic uncertainty. 


\begin{tabular}{|c|c|c|c|}
\hline & $\left(1-\epsilon_{Z v t x}^{\text {data }}\right)$ & $\left(1-\epsilon_{\text {Zvtx }}^{M C}\right)$ & $\epsilon_{\text {Zvtx }}^{\text {Data }} / \epsilon_{\text {Zvtx }}^{M C}$ \\
\hline$|Z v t x|<60 \mathrm{~cm}$ & $0.036 \pm 0.001$ & $0.018 \pm 0.001$ & $0.982 \pm 0.001$ \\
$|Z v t x|<60 \mathrm{~cm}$ w/ diff. bkg & $0.036 \pm 0.001$ & $0.018 \pm 0.001$ & $0.981 \pm 0.001$ \\
no Zvtx cut & $0.042 \pm 0.002$ & $0.019 \pm 0.001$ & $0.977 \pm 0.002$ \\
no Zvtx cut w/ diff. bkg & $0.041 \pm 0.001$ & $0.019 \pm 0.001$ & $0.978 \pm 0.001$ \\
$66<M_{Z}<116 \mathrm{GeV}$ & $0.035 \pm 0.001$ & $0.017 \pm 0.001$ & $0.982 \pm 0.001$ \\
\hline
\end{tabular}

Table 5.2: The reconstructed ZVertex inefficiency and scale factor. Here, $\epsilon_{Z \text { Zvtx }}^{\text {data }}$ is the efficiency for the ZVertex reconstruction in data, and $\epsilon_{Z v t x}^{M C}$ is the efficiency in the MC.
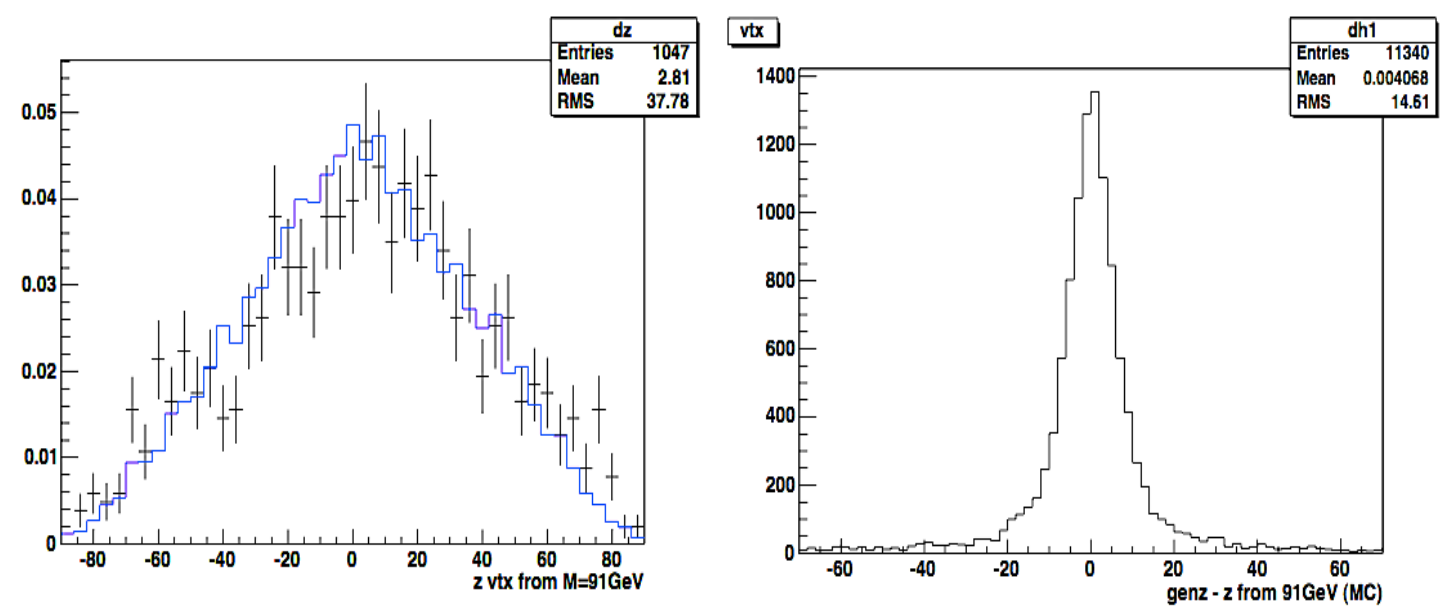

Figure 5.5: The ZVertex determined by the Z mass constrained method. The plot in left side shows the ZVertex distribution determined by Z/dilepton mass constrained method. The black crosses are the data and the blue histogram is the MC. The plot on right shows the difference between the generated ZVertex and the ZVertex determined by Z/dilepton mass constrained method for MC events. 

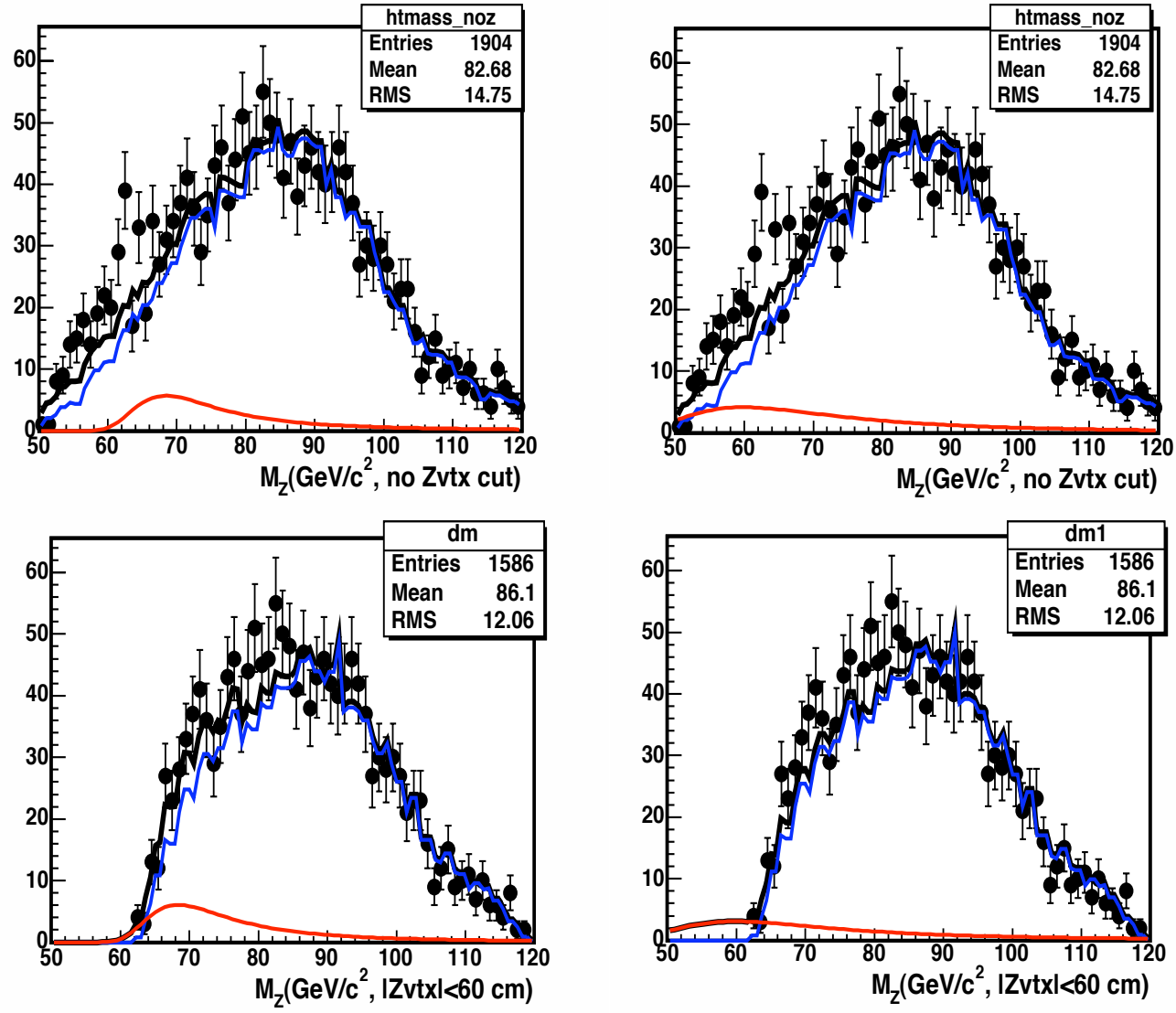

Figure 5.6: The $\mathrm{Z}$ mass distribution of the events without a reconstructed ZVertex in data. The ZVertex in the events is set to be zero in order to to reconstruct the $\mathrm{Z} /$ dilepton invariant mass. Top two plots do not have a Zvertex requirement and the bottom two plots require $\mid Z V$ ertex $\mid<60 \mathrm{~cm}$. The ZVertex used for $\mid Z V$ ertex $\mid<$ $60 \mathrm{~cm}$ requirement is determined by the $\mathrm{Z} /$ dilepton mass constrained method. The plots on the left use the background shape from events which do not have ZVertex information, and the plots on the right use the background shape from events which have Zvertex information. The black line is the combined signal and background histogram. The blue line is signal, and the red line is the background contribution. 

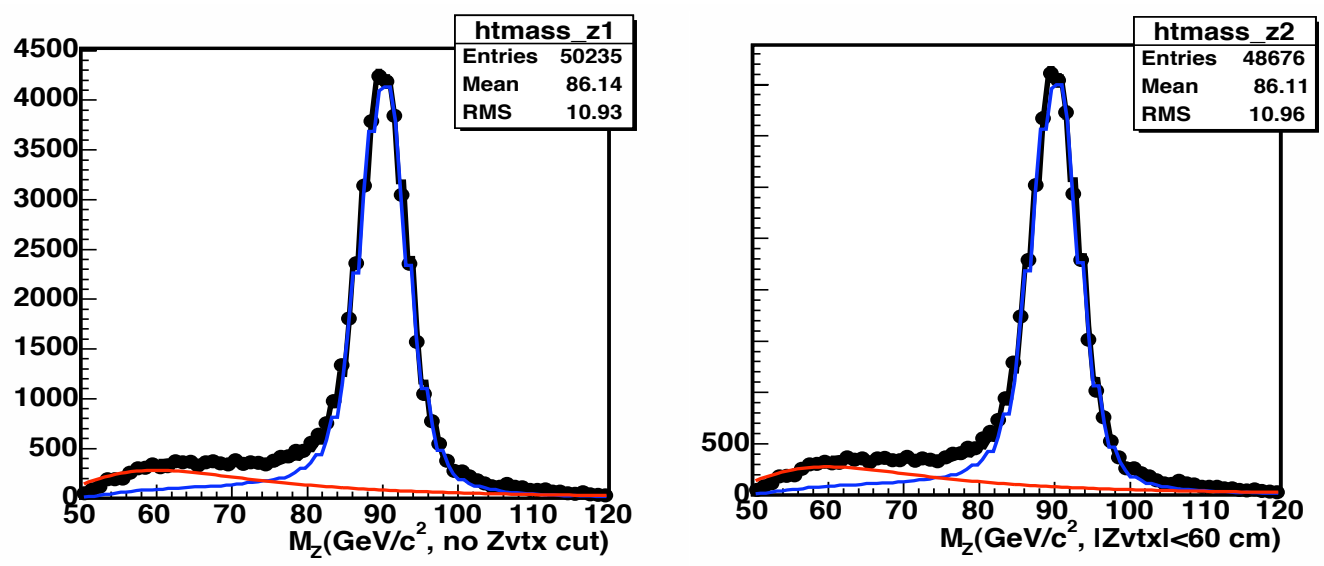

Figure 5.7: The Z mass distribution for events with a reconstructed ZVertex in data. The plot on the left shows Z/dilepton events without any requirement on ZVertex. The plot on the right shows the Z/dilepton events with $\mid Z V$ ertex $\mid<60 \mathrm{~cm}$. The black line is the combined signal and background histogram. The blue line is the signal and the red line is the background contribution.

\subsubsection{ZVertex Selection}

The phoenix track is sensitive to the ZVertex position because the phoenix track is reconstructed by constraining the track to start at the ZVertex location and end at the position of the centroid of the EM shower in the PES calorimeter strips. Therefore, assigning the correct ZVertex to the track is important, especially when there are the multiple ZVertices in an event. In the case of events for which there is a track associated with the electron, the $z_{0}$ position of the track is used for the reconstruction of the $\mathrm{Z} /$ dilepton invariant mass. If both electrons have associated tracks, the $z_{0}$ of the highest $P_{T}$ track is selected for the ZVertex. Even if the event does not have any track associated with the electron, the ZVertex of the event can be reconstructed by one of the three ZVertex finding algorithms.

There are three different vertex finding algorithms: The maximum SumPt method, 
the highest class method, and the Z/dilepton mass constrained method. These three different algorithms are compared using MC events. The maximum SumPt algorithm determines the ZVertex that has the maximum value for the sum of the absolute value of the $P_{T}$ of all tracks associated with the reconstructed ZVertex. The highest class algorithm compares the quality of the reconstructed ZVertex between different ZVertex reconstruction choices. The Z/dilepton mass constrained method finds the ZVertex for which the Z/dilepton reconstructed invariant mass is the closest to $91 \mathrm{GeV} / \mathrm{c}^{2}$.

The fraction of events for which there is more than a $10 \mathrm{~cm}$ difference between the generated ZVertex and the reconstructed ZVertex as determined by each algorithm is used to determine how often the algorithm fails to find the correct ZVertex. We find that the maximum SumPt, the highest class, and the $\mathrm{Z}$ mass constrained method give a ZVertex which is located more than $10 \mathrm{~cm}$ from the true vertex and these fraction of events is $47.18 \pm 0.43 \%, 46.40 \pm 0.43 \%$, and $10.25 \pm 0.15 \%$, respectively. The Z mass constrained method selects the correct ZVertex more often than the other two methods. Therefore, the $\mathrm{Z}$ mass constrained algorithm is used for determining the ZVertex in no-track events with multiple ZVertices. If the event has only one ZVertex, that ZVertex is used. In this case, only $11.96 \pm 0.19 \%$ of events have a reconstructed ZVertex which is more than $10 \mathrm{~cm}$ away from the generated ZVertex. For events for which there is an electron which is associated with a track, the correct ZVertex is found with very high efficiency. Here, only $0.36 \pm 0.01 \%$ of events have a reconstructed ZVertex which is more than $10 \mathrm{~cm}$ away from the generated ZVertex. Figure 5.8 shows the difference between the generated and reconstructed ZVertex for different categories of events. 
(a)
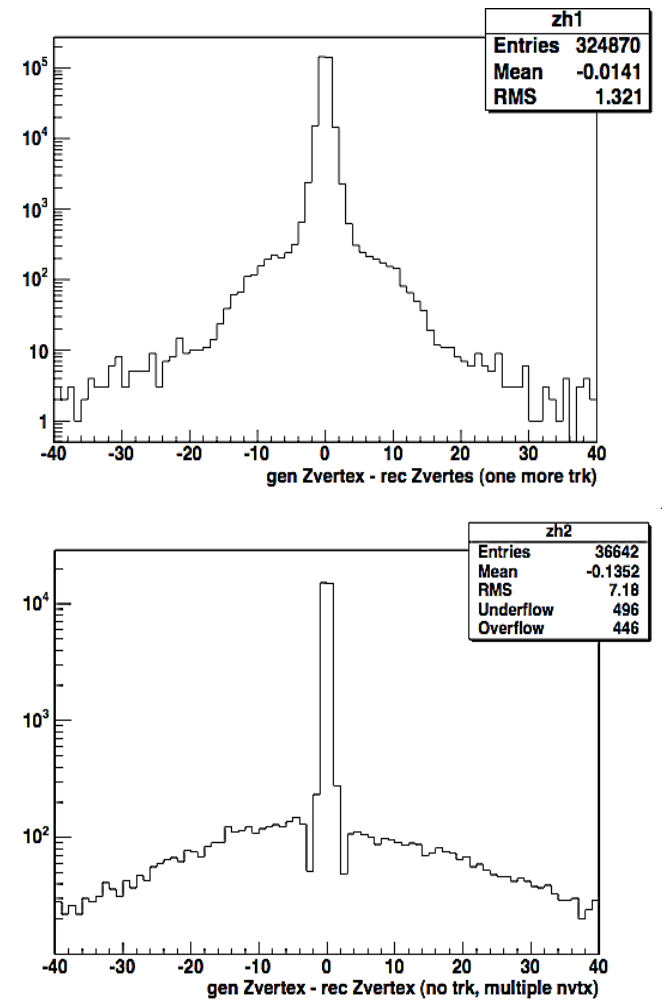

(b)
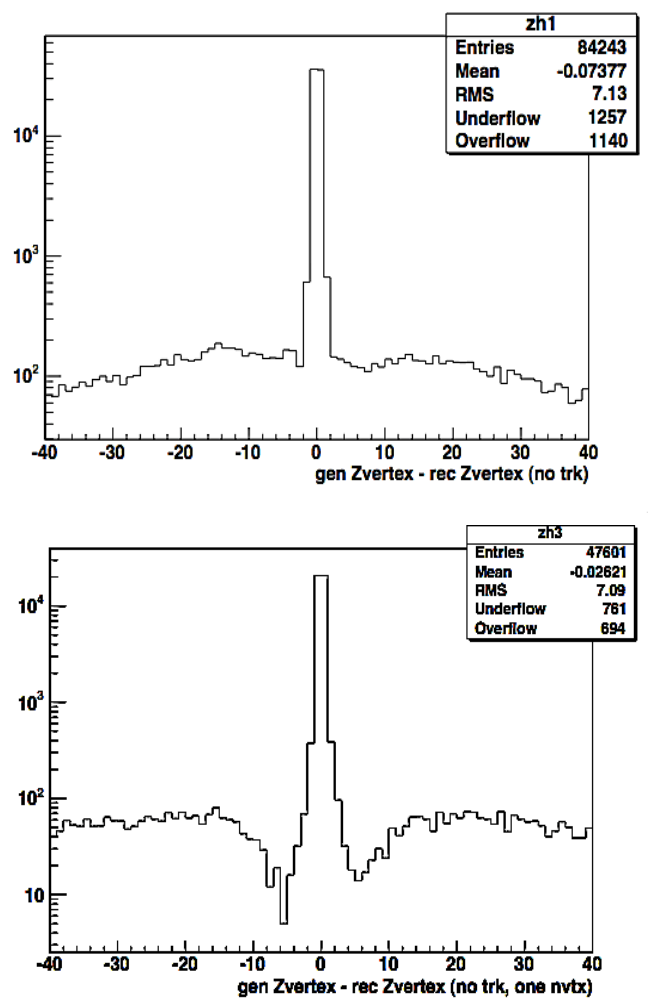

(d)

Figure 5.8: The difference between the generated ZVertex and the reconstructed ZVertex as determined by the ZVertex finding algorithm for different event categories. (a) Events with one or more tracks associated with an electron (b) Events with no track associated with an electron (c) Events with no track associated with an electron for which there are multiple ZVertices. (d) Events with no track associated with an elecron for which there is only one reconstructed ZVertex. Note that Plot (b) is equivalent to Plots (c) and (d) combined. 


\subsubsection{Phoenix Tracking Efficiency Dependence on Instanta- neous Luminosity}

During the period between February, 2006 to June, 2006 there was an accelerator shutdown to make improvements aimed at increasing the instantaneous luminosity. After this shutdown, the instantaneous luminosity at the start of a store increased up to $3 \times 10^{32} \mathrm{~cm}^{-2} \mathrm{sec}^{-1}$. The instantaneous luminosity affects the tracking efficiency because at higher instantaneous luminosity there are more events with multiple interactions during a single beam crossing. Multiple interactions affect the ZVertex reconstruction and result in a lower silicon phoenix tracking efficiency. Therefore, the phoenix tracking efficiency is measured as a function of instantaneous luminosity. The tracking efficiency and fraction of no-track events (as described below) are determined as a function of the number of ZVertices (NVertices) in the event, which increase as the instantaneous luminosity increases.

\subsubsection{Events with a ZVertex but No Track}

Events with a reconstructed ZVertex for which there are no tracks associated with the electrons are treated separately from the events which have one or more electron associated tracks. In the $\mathrm{Z}(\mathrm{PP})$ events without any tracks associated with the electrons in the calorimeter, either the electrons do not have reconstructable tracks or there has been a failure in the plug region phoenix tracker. If the wrong ZVertex is selected by the tracking algorithm, then no phoenix track is found in the the silicon tracker.

The track fiduciality is defined as the expected number of silicon layers that the electron traverses based on its geometry ( $\eta$ and ZVertex). Events with only one electron associated track in the tracking fiducial volume may be reconstructed differently 
than events for which that are two electrons with associated tracks in the tracking fiducial volume. For example, Z/Drell-Yan events with both electrons in the track fiducial volume have a reconstructed ZVertex distribution which is close to the correct vertex. This is because the two electron tracks originate from the same vertex and increase the probability that the vertex is reconstructed correctly.

The $\mathrm{Z} /$ dielectron rapidity is also correlated with the ZVertex and $\eta$ position in the PES calorimeter. Therefore, the fraction of no-track events is determined separately for each of the two categories, (a) events with one electron in the tracking volume and (b) events with both electrons in the tracking fiducial volume. The fraction of events with one electron in the fiducial region $\left(F_{1}\right)$, and the fraction of the events with two electrons in the fiducial region $\left(F_{2}\right)$, are measured for both data and $\mathrm{MC}$ events. The scale factors which are the ratio of data to $\mathrm{MC}\left(S F_{1}\right.$ and $\left.S F_{2}\right)$ for each category are also determined.

The $F_{1}\left(S F_{1}\right)$ and $F_{2}\left(S F_{2}\right)$ ratios are defined as:

$$
\begin{gathered}
F_{1}=\frac{Z(P P) \text { with one electron in track fiduciality }}{Z(P P) \text { with one and more electron in track fiduciality }} \\
F_{2}=\frac{Z(P P) \text { with both electrons in track fiduciality }}{Z(P P) \text { with one and more electron in track fiduciality }} \\
\qquad S F_{1}=\frac{F_{1}(\text { data })}{F_{1}(M C)} \quad ; \quad S F_{2}=\frac{F_{2}(\text { data })}{F_{2}(M C)}
\end{gathered}
$$

The background contamination in the data is subtracted by fitting the $\mathrm{Z} /$ dielectron mass distribution. Figure 5.9 shows the mass distributions for both event categories. The fractions, $F_{1}$ and $F_{2}$ are measured for events with one, two, three or more collision vertices to correctly model this fraction at high luminosity. Table 5.3 summarizes the 

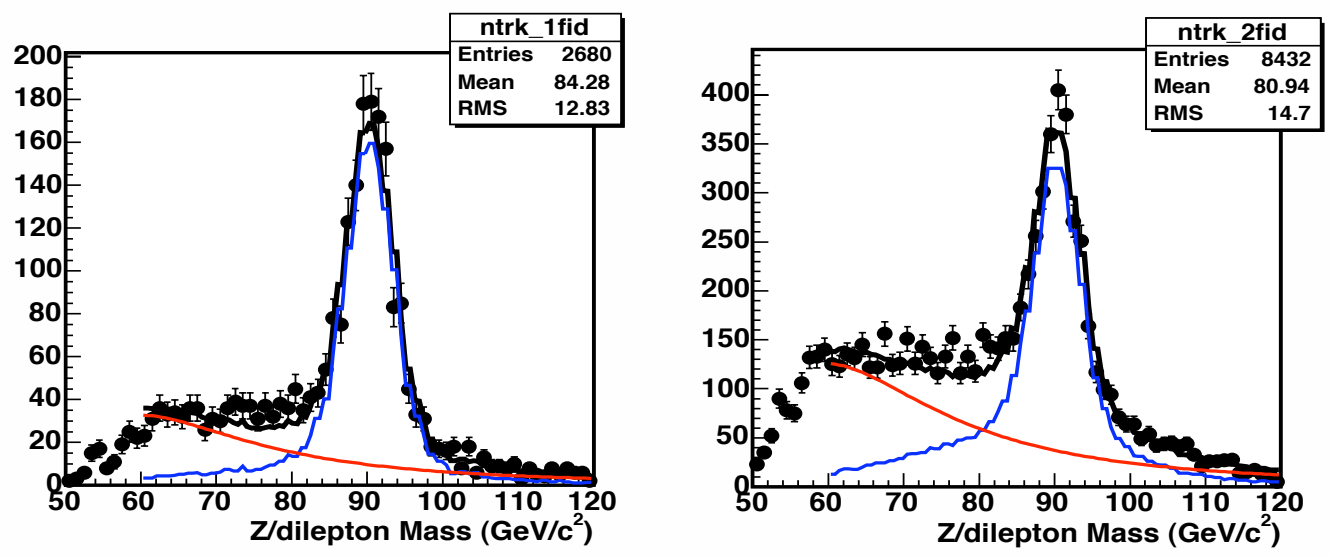

Figure 5.9: The Z/dilepton mass distribution for no-track events with a ZVertex. The plot on the left is the Z/dilepton mass distribution for events which have only one electron in the tracking fiducial volume. The plot in the right is the Z/dilepton mass distribution for events with both electrons in the track fiducial volume. The blue line is signal and the red line is the background contribution. The black line is the combined signal and background contribution.

values of $F_{1}, F_{2}$ for the data and for the $\mathrm{MC}$ sample and the scale factors $S F_{1}$ and $S F_{2}$ for each collision vertex topology.

\subsubsection{Events with Tracks}

Events in the $\mathrm{Z}(\mathrm{PP})$ topology with one and more electron associated tracks tend to have a correctly reconstructed ZVertex due to the presence of those electron tracks. For this case, most of the tracking inefficiency originated from the hit finding inefficiency in each silicon layer, and not from an incorrect ZVertex assignment. The phoenix tracking efficiency is estimated as a function of the expected number of silicon layers that the electron traverses $\left(N_{\text {sil }}^{\text {expected }}\right)$. Here, $N_{\text {sil }}^{\text {expected }}$ is derived from the ZVertex, $\eta_{\text {Det }}$, and silicon tracker geometry. Therefore, it reflects any ZVertex and $\eta_{\text {Det }}$ correlation. 
(a) NVertices $=1$

\begin{tabular}{|c|c|c|c|}
\hline & data & MC & SF \\
\hline$F_{1}$ & $0.036 \pm 0.003$ & $0.036 \pm 0.001$ & $1.012 \pm 0.072$ \\
$F_{2}$ & $0.044 \pm 0.006$ & $0.043 \pm 0.001$ & $1.004 \pm 0.128$ \\
\hline
\end{tabular}

(b) NVertices $=2$

\begin{tabular}{|c|c|c|c|}
\hline & data & MC & SF \\
\hline$F_{1}$ & $0.057 \pm 0.003$ & $0.047 \pm 0.001$ & $1.225 \pm 0.070$ \\
$F_{2}$ & $0.141 \pm 0.006$ & $0.099 \pm 0.001$ & $1.427 \pm 0.059$ \\
\hline
\end{tabular}

(c) NVertices $\geq 3$

\begin{tabular}{|c|c|c|c|}
\hline & data & MC & SF \\
\hline$F_{1}$ & $0.057 \pm 0.004$ & $0.052 \pm 0.001$ & $1.095 \pm 0.070$ \\
$F_{2}$ & $0.202 \pm 0.007$ & $0.142 \pm 0.001$ & $1.417 \pm 0.050$ \\
\hline
\end{tabular}

Table 5.3: The fraction of 1 track-fiducial electrons and 2 track-fiducial electrons in data and MC for no track events. SF is the scale factor for these fractions (ratio of data/MC). NVertices is the number of $p \bar{p}$ collision vertices found in the event.

The comparison of the event fractions as a function of $N_{\text {sil }}^{\text {expected }}$ between data and $\mathrm{MC}$ is a check on how well the MC simulates the data for both the ZVertex and $\eta_{D e t}$ distributions. If either the ZVertex or the $\eta_{D e t}$ distributions are not simulated well in $\mathrm{MC}$, the event fractions as a function of $N_{\text {sil }}^{\text {expected }}$ in $\mathrm{MC}$ will not match the data. Figure 5.10 shows that there is a good agreement between data and MC for the fraction of events with different $N_{\text {sil }}^{\text {expected }}$ topologies. To estimate the phoenix tracking efficiency, the $\mathrm{Z}(\mathrm{PP})$ topology sample is used. All electron identification requirements are applied to this sample to reduce background. In addition, one electron is also required to have a track to further reduce background and provide a good ZVertex in the event. The efficiency is measured with the other leg (target leg) on which the tracking requirement is not applied. To avoid any bias in the selection 


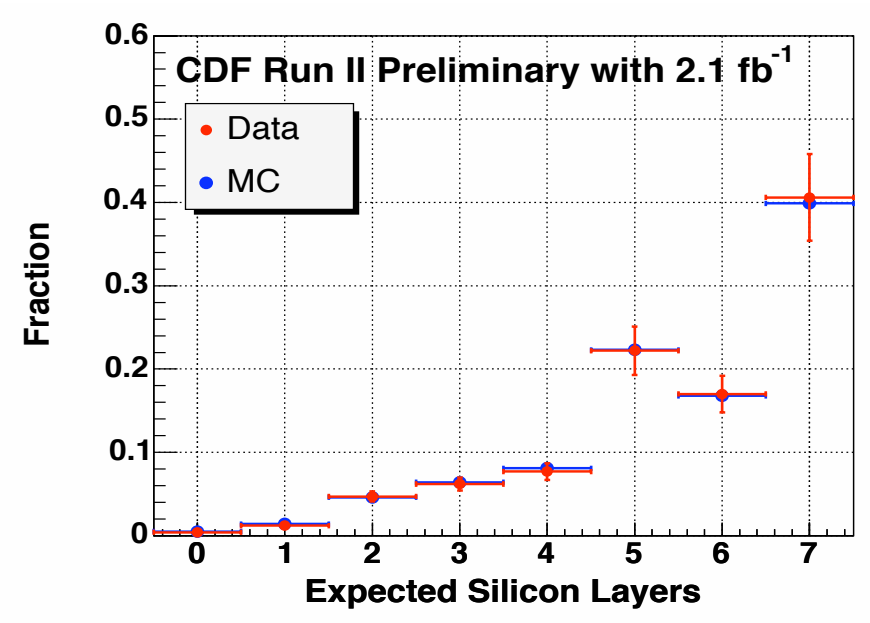

Figure 5.10: The fraction of events in each fiducial range. The plot shows good agreement between data and MC for the fraction of the events versus $N_{\text {sil }}^{\text {expected }}$.

of the target leg, the target leg is selected randomly and a phoenix track is required for the other leg(tagged leg). The phoenix tracking efficiency for electrons is defined as

$$
\epsilon=\frac{\text { Number of } Z(P P) \text { events with track in target electron }}{\text { Number of } Z(P P) \text { events }}
$$

The efficiency $(\epsilon)$ is measured as a function of $N_{\text {sil }}^{\text {expected }}$ and also versus NVertices to include ZVertex, $\eta_{D e t}$, and instantaneous luminosity dependence. The background is subtracted using the $\mathrm{Z} /$ dilepon mass fit method. Table 5.5 shows the phoenix tracking efficiency for electrons and the scale factor of the efficiency $\left(\mathrm{S}=\epsilon^{\text {Data }} / \epsilon^{M C}\right)$ versus $N_{\text {sil }}^{\text {expected }}$ and NVertices. A scale factor, $\mathrm{S}$ is applied to the MC to simulate that correct tracking efficiency as a function of boson rapidity.

\subsubsection{Phoenix Tracking Efficiency versus Boson Rapidity}

The efficiency of having one or more phoenix tracks for $\mathrm{Z}(\mathrm{PP})$ events is determined from the MC sample. MC sample is tuned to agree with data by applying the scale 


\begin{tabular}{|c|c|c|}
\hline expected silicon layers $\left(N_{\text {sil }}^{\text {expected }}\right)$ & data & $\mathrm{MC}$ \\
\hline 0 & $0.004 \pm 0.001$ & 0.005 \\
1 & $0.012 \pm 0.002$ & 0.014 \\
2 & $0.047 \pm 0.006$ & 0.046 \\
3 & $0.062 \pm 0.008$ & 0.064 \\
4 & $0.077 \pm 0.010$ & 0.081 \\
5 & $0.222 \pm 0.029$ & 0.223 \\
6 & $0.170 \pm 0.022$ & 0.168 \\
7 and more & $0.406 \pm 0.052$ & 0.399 \\
\hline
\end{tabular}

Table 5.4: The fraction and the tracking efficiency as a function of the expected number of silicon layers for data and MC.

factors for the event fraction and tracking efficiency (SF and S), as described in section 5.2.4 and 5.2.5, to MC events on an event by event basis (as an event weight). If the electron does not have a phoenix track, the scale factor for the inefficiency $\left(S I=\left(1-\epsilon^{\text {Data }}\right) /\left(1-\epsilon^{M C}\right)\right)$ is also applied to conserve the total number of events. The event weighting factors that are applied include the dependence on NVertices and $N_{\text {sil }}^{\text {expected }}$. Table 5.6 summarizes the weighting factors for events in each category. The tuned MC sample (corrected by the event weighting factors) is used to estimate the efficiency of having one or two tracks as a function of the boson rapidity. The efficiency is defined as

$$
\epsilon_{\text {Track }}^{P P}(y)=\frac{\text { Number of } Z(P P) \text { with one or more tracks }}{\text { Number of } Z(P P) \text { with track fiduciality }}
$$

where the track fiducial requirement for $\mathrm{Z}(\mathrm{PP})$ events is that at least one electron has $N_{\text {sil }}^{\text {expected }} \geq 3$, and the event has a reconstructed ZVertex. The Z/Drell-Yan mass window, $66<M<116 \mathrm{GeV} / \mathrm{c}^{2}$, is required in the determination of rapidity dependence of the efficiency. Figure 5.11 shows the efficiency as a function of Z/dielectron rapidity $\left(y_{Z}\right)$. The overall tracking efficiency in the $\mathrm{Z}(\mathrm{PP})$ region is measured to be 
(a) $N$ Vertices $=1$

\begin{tabular}{|c|c|c|c|}
\hline$N_{\text {sil }}^{\text {expected }}$ & $\epsilon^{\text {Data }}$ & $\epsilon^{M C}$ & $\mathrm{~S}=\epsilon^{\text {Data }} / \epsilon^{M C}$ \\
\hline 3 & $0.384 \pm 0.027$ & $0.371 \pm 0.003$ & $1.037 \pm 0.075$ \\
4 & $0.819 \pm 0.041$ & $0.744 \pm 0.003$ & $1.101 \pm 0.055$ \\
5 & $0.936 \pm 0.027$ & $0.905 \pm 0.001$ & $1.034 \pm 0.029$ \\
6 & $0.972 \pm 0.031$ & $0.960 \pm 0.001$ & $1.012 \pm 0.032$ \\
7 and more & $0.978 \pm 0.021$ & $0.981 \pm 0.001$ & $0.997 \pm 0.021$ \\
\hline
\end{tabular}

(b) NVertices $=2$

\begin{tabular}{|c|c|c|c|}
\hline$N_{\text {sil }}^{\text {expected }}$ & $\epsilon^{\text {Data }}$ & $\epsilon^{M C}$ & $\mathrm{~S}=\epsilon^{\text {Data }} / \epsilon^{M C}$ \\
\hline 3 & $0.409 \pm 0.044$ & $0.365 \pm 0.005$ & $1.119 \pm 0.122$ \\
4 & $0.800 \pm 0.058$ & $0.735 \pm 0.004$ & $1.088 \pm 0.079$ \\
5 & $0.917 \pm 0.032$ & $0.892 \pm 0.002$ & $1.029 \pm 0.036$ \\
6 & $0.960 \pm 0.043$ & $0.959 \pm 0.001$ & $1.001 \pm 0.045$ \\
7 and more & $0.978 \pm 0.025$ & $0.978 \pm 0.001$ & $1.000 \pm 0.025$ \\
\hline
\end{tabular}

(c) NVertices $=3$

\begin{tabular}{|c|c|c|c|}
\hline$N_{\text {sil }}^{\text {expected }}$ & $\epsilon^{\text {Data }}$ & $\epsilon^{M C}$ & $\mathrm{~S}=\epsilon^{\text {Data }} / \epsilon^{M C}$ \\
\hline 3 & $0.343 \pm 0.062$ & $0.355 \pm 0.007$ & $0.965 \pm 0.175$ \\
4 & $0.784 \pm 0.076$ & $0.715 \pm 0.006$ & $1.097 \pm 0.107$ \\
5 & $0.882 \pm 0.047$ & $0.878 \pm 0.002$ & $1.005 \pm 0.053$ \\
6 & $0.945 \pm 0.045$ & $0.953 \pm 0.002$ & $0.992 \pm 0.047$ \\
7 and more & $0.956 \pm 0.029$ & $0.974 \pm 0.001$ & $0.981 \pm 0.030$ \\
\hline
\end{tabular}

Table 5.5: The phoenix tracking efficiency for electrons. The tables summarize the tracking efficiency for electrons versus $N_{\text {sil }}^{\text {expected }}$ and NVertices, as measured in data and MC. Also shown are the scale factors (data/MC) for the tracking efficiencies. 


\begin{tabular}{|l|c|}
\hline Event Case & Weighting Factor \\
\hline $\begin{array}{l}\text { No Track } \\
\text { One Electron in } N_{\text {sil }}^{\text {expected }} \geq 3\end{array}$ & $S F_{1}(N$ Vertices $)$ - Table 5.3 \\
\hline $\begin{array}{l}\text { No Track } \\
\text { Two Electron in } N_{\text {sil }}^{\text {expected }} \geq 3\end{array}$ & $S F_{2}(N$ Vertices $)$ - Table 5.3 \\
\hline One Phoenix Track & $S\left(n, N_{\text {sil }}^{\text {expected }}(e 1)\right) \times S I\left(n, N_{\text {sil }}^{\text {expected }}(e 2)\right)$ \\
Phoenix Electron : $N_{\text {sil }}^{\text {expected }}(e 1)$ & where NVertices $=\mathrm{n}$ \\
Plug Electron $: N_{\text {sil }}^{\text {expected }}(e 2)$ & \\
\hline Two Phoenix Track & $S\left(n, N_{\text {sil }}^{\text {expected }}(e 1)\right) \times S\left(n, N_{\text {sil }}^{\text {expected }}(e 2)\right)$ \\
Phoenix Electron 1: $N_{\text {sil }}^{\text {expected }}(e 1)$ & where NVertices $=\mathrm{n}$ \\
Phoenix Electron 2: $N_{\text {sil }}^{\text {expected }}(e 2)$ & \\
\hline
\end{tabular}

Table 5.6: The weighting factors for the tracking efficiency. Here, Z(PP) events include four cases (after kinematic selection). Each case has a different weighting factor. The weighting factor in each case is applied to tune the MC to have the same efficiency and event fractions as the data.

$0.847 \pm 0.001$

\subsection{Electron Identification Efficiency}

Electrons are selected by requiring kinematic and electron identification selection criteria described in Tables 3.1 and 3.2. Since the kinematic selections such $E_{T}$, fiduciality, Track $z_{0}$, and Track $P_{T}$ (only for the central electron) are already included in the acceptance, the electron identification efficiency does not include the efficiency of these kinematic cuts (see acceptance chapter 5). The electron identification(ID) criteria are the remaining cuts used to reject background and select the electron sample. Note that the electron ID efficiency also does not include the tracking efficiency. 


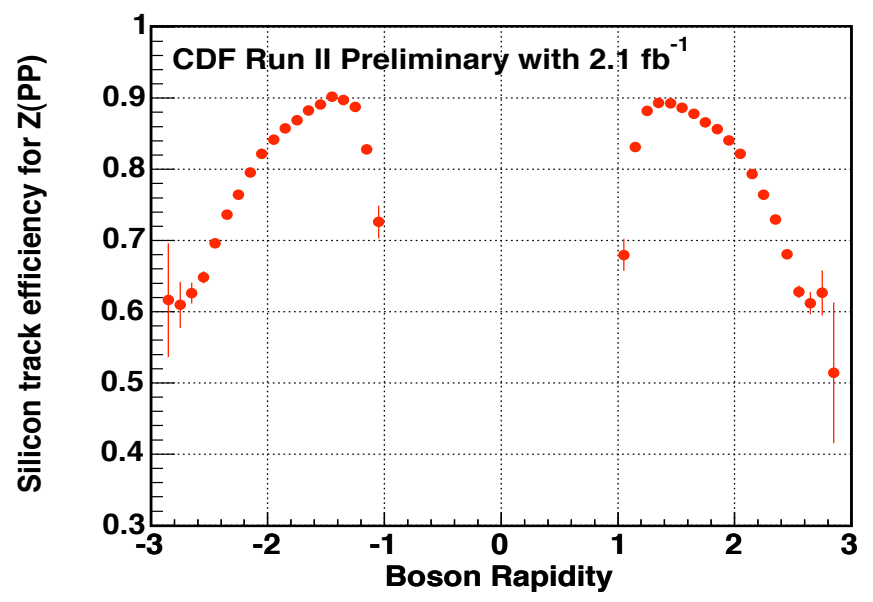

Figure 5.11: The tracking efficiency for Z(PP) events. The efficiency of having one or more phoenix tracking in $\mathrm{Z}(\mathrm{PP})$ is determined as a function of boson rapidity $\left(y_{Z}\right)$ using the tuned MC sample.

\subsubsection{Electron Identification Efficiency versus Time}

The instantaneous luminosity changed with time, especially for runs after June 2006, for which the increase is up to $3.0 \times 10^{12} \mathrm{~cm}^{-2} \mathrm{sec}^{-1}$. The higher instantaneous luminosity affects the electron ID efficiency because of additional underlying events from multiple interactions in the same beam crossing. Figure 5.12 shows the number of events divided by the total integrated luminosity in each run period. The number of events in the first run period is normalized to be 1 . The event rate decreases with time and the effect in the $\mathrm{Z}(\mathrm{PP})$ sample is larger than for the other event topologies. For $\mathrm{Z}(\mathrm{PP})$, the drop in the event rate is $\sim 20 \%$. The $\chi^{2}$ of the shower shape requirement $P E M \chi_{3 \times 3}^{2}$ is the main source of the decreasing efficiency for events in the $\mathrm{Z}(\mathrm{PP})$ sample. Therefore, the electron ID efficiency determination includes a time dependence as described below. 

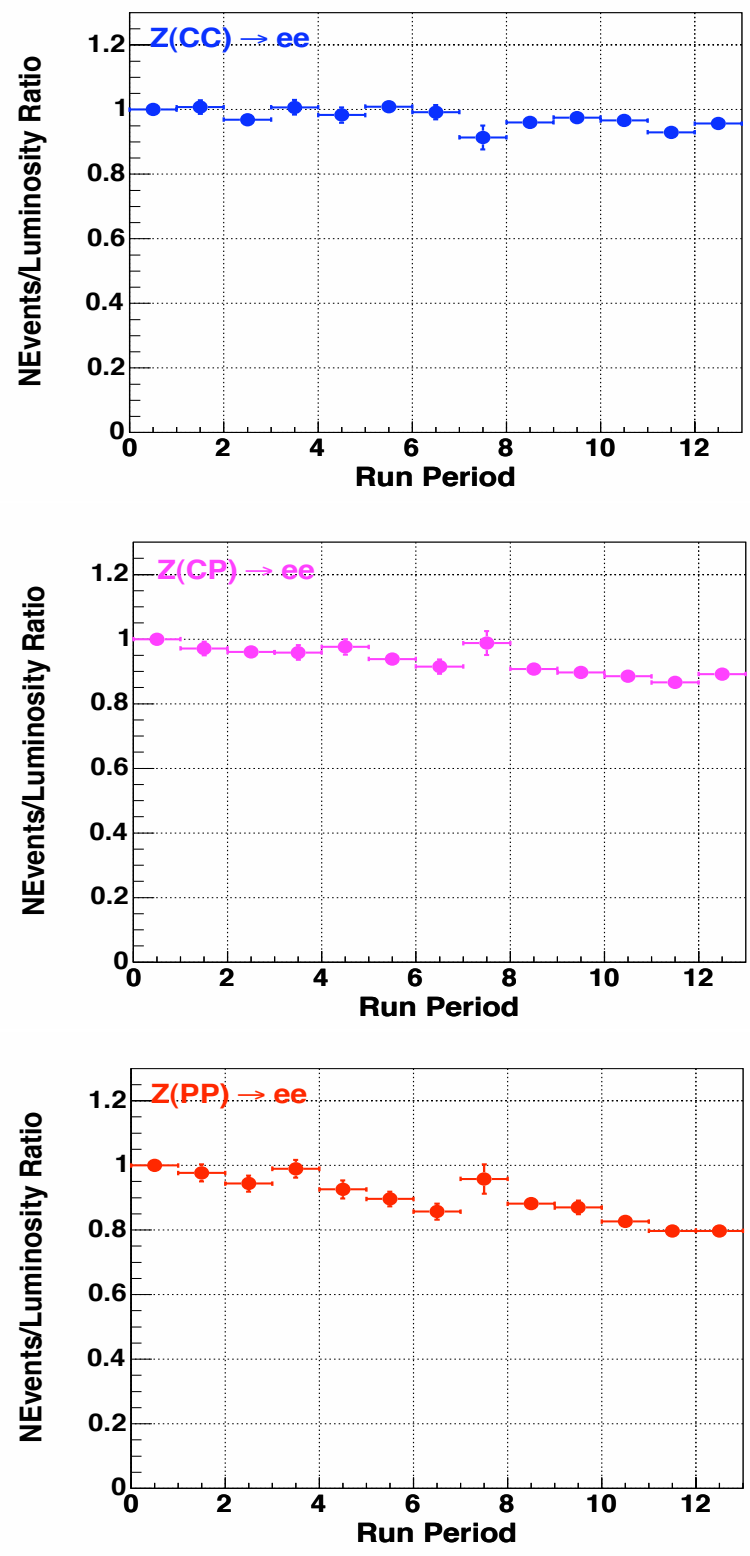

Figure 5.12: The event rate in each run period. The number of events divided by the total integrated luminosity is measured in each run period. The event rate decreases in time due to the lower efficiency at high luminosity. 


\subsubsection{Central Electron Identification Efficiency}

The central electron ID efficiency is measured with $\mathrm{Z}(\mathrm{CC})$ events. The tight central electron selection cuts include both the shower maximum position detector and track based identification requirements. In parts of the detector we observe an efficiency drop dependence in $\eta_{D e t}$, especially in the shower max wire support region in $\mathrm{z}$. However, this effect is not modeled well in the MC. The efficiency in MC is relatively flat as a function of the track $\mathrm{z}$ position. The central electron efficiency is measured as a function of the track $\mathrm{z}$ in data and $\mathrm{MC}$. Here, $\mathrm{Z}(\mathrm{CC})$ events are required to have one tight electron and one EM object which passes the kinematic selection cuts. The tight electron is selected as the tagged leg to reduce background, and the second EM object is selected as a target leg which is used to estimate the efficiency. The target leg is randomly selected in $E_{T}$ to avoid an $E_{T}$ bias. The efficiency is measured as a function of the track $\mathrm{z}$ of the target leg to include detector geometry effects. The tight or loose electron efficiency $\left(\epsilon_{T}\right.$ or $\left.\epsilon_{L}\right)$ is measured as

$$
\epsilon_{T(L)}(\operatorname{track} z)=\frac{N_{T T(T L)}(\operatorname{track} z)}{N_{T K}(\operatorname{track} z)}
$$

where $N_{T T(T L)}$ is the number of events for which the target leg passes the tight(loose) ID cuts and, $N_{T K}$ is the number of events for which the target leg passes the kinematic selection cuts. The background is estimated by the isolation extrapolation method as described in section 4 . The background is subtracted assuming that the background is flat versus the track $z$. The tight and loose central electron efficiencies are measured for data and MC, respectively and the scale factor data/MC is determined versus track

z. The scale factor is applied to MC events to determine the efficiency versus $\mathrm{Z}$ boson 

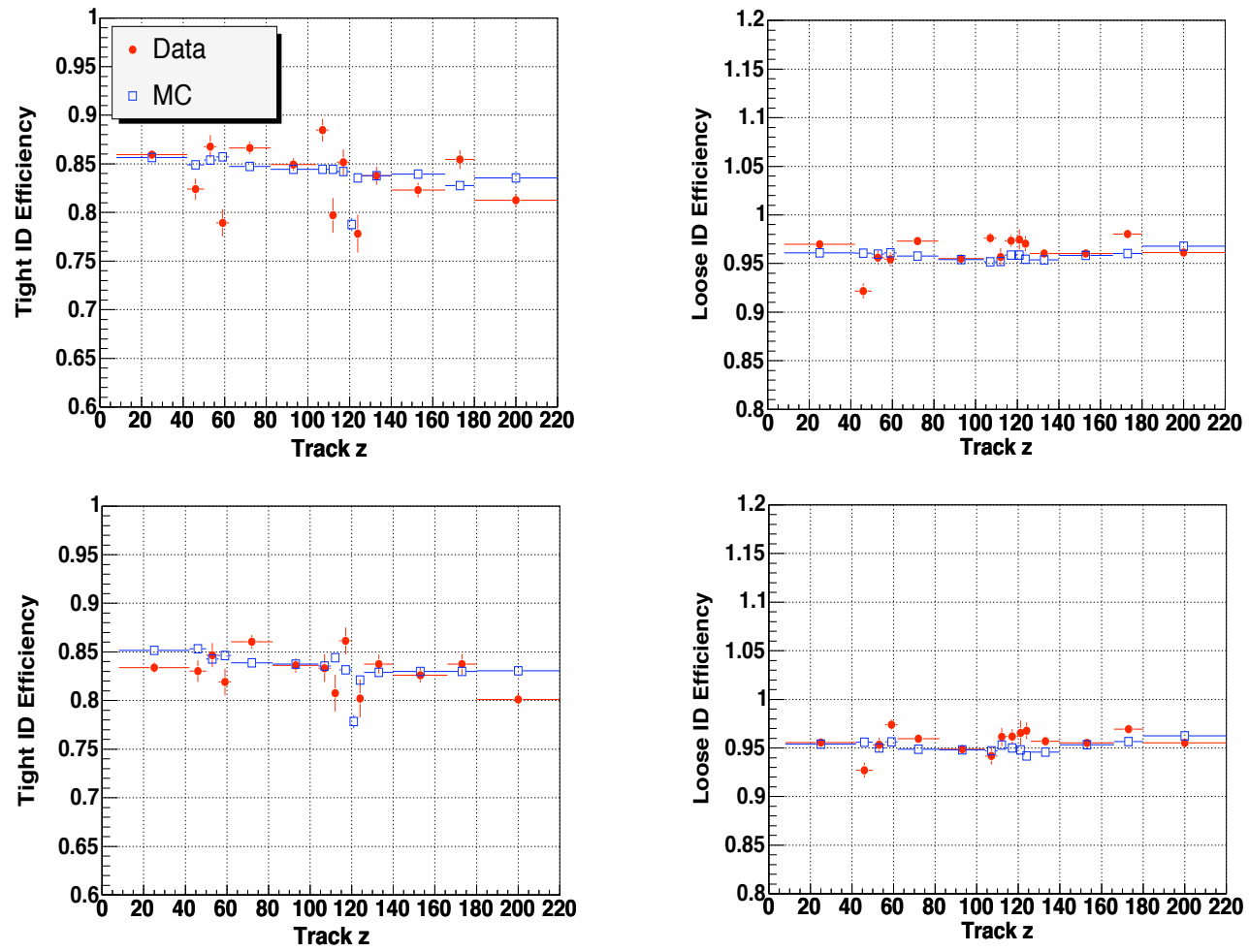

Figure 5.13: The central electron ID efficiency. The plots on the top show the tight and loose central electron ID efficiency for run periods 0 to 7 . The plots on the bottom show the efficiencies for run periods 8 to 12 .

rapidity. The efficiency is measured for data collected before June 2006 (period 0 to 7), and after June 2006 (period 8 to 12). The instantaneous luminosity was increased significantly after June 2006. The efficiencies for central electrons and the scale factors of the efficiency (data/MC) are shown in Figure 5.13. The background level in the sample before the ID selection on the target leg is $2 \%$. The overall efficiency for tight central electrons is $0.837 \pm 0.002$, and for loose central electrons it is $0.961 \pm 0.001$. The central electron ID efficiency measured using $\mathrm{Z}(\mathrm{CC})$ events is also applied to the central electron of events in the $\mathrm{Z}(\mathrm{CP})$ topology. 


\subsubsection{Plug Electron Identification Efficiency in $\mathrm{Z}(\mathrm{CP})$}

The plug electron ID efficiency is measured using $\mathrm{Z}(\mathrm{CP})$ events. The central electron is required to pass tight selection cuts to reject background in the sample. The plug electron is used as the target leg to determine the plug electron ID efficiency. Although the plug electron ID efficiency has an $\eta$ dependence, it is difficult to measure the efficiency as a function of $\eta$ because the fraction of background changes with $\eta$ and the estimate of the background fraction versus $\eta$ has large errors. Therefore, first the overall efficiency is measured versus run period in data and in MC. A global scale factor for the total efficiency (data/MC) is applied to the MC to tune the simulation and the tuned MC is used to determine the efficiency dependence versus $\eta$ assuming that the dependence in $\eta$ is well modeled in the simulation. The efficiency is measured as

$$
\epsilon_{P}^{C P}(\text { run })=\frac{N_{T P}(\text { run })}{N_{T K}(\text { run })}
$$

where $N_{T P}$ is the number of events with a tight central electron and also with a plug electron which satisfies all plug ID requirements (kinematic and ID cuts), and $N_{T K}$ is the number of events with a tight central electron and a plug EM object which satisfies the kinematic selection cuts. The background is estimated using the Z/dilepton mass fit method. Here the signal template from the MC simulation is used, and the background template is derived from data. For the background sample selection, the background selection anti-electron cuts described in Table 4.1 are required in data. The plug electron ID efficiency versus run period (measured with $\mathrm{Z}(\mathrm{CP})$ events) is shown in Figure 5.14. The overall efficiency is $0.830 \pm 0.004$. 


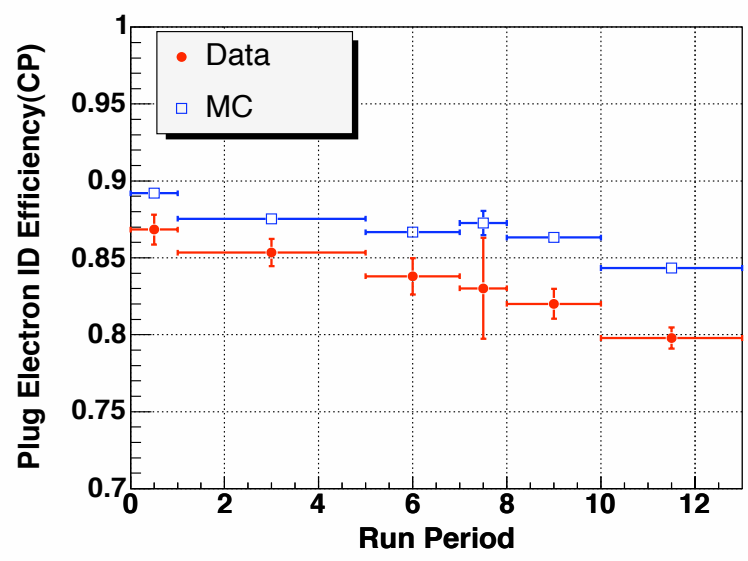

Figure 5.14: The plug electron ID efficiency for $\mathrm{Z}(\mathrm{CP})$ events. The efficiency is measured versus run period.

\subsubsection{Plug Electron Identification Efficiency in $\mathrm{Z}(\mathrm{PP})$ Events}

The plug electron ID efficiency for $\mathrm{Z}(\mathrm{PP})$ is measured using the $\mathrm{Z}(\mathrm{PP})$ sample. The plug electron ID efficiency is found to be correlated with the tracking efficiency. Therefore, the ID efficiency is measured separately for electrons associated with a silicon phoenix track, and for electrons without a track. In addition, the plug ID efficiency is decreasing in time, which is described in section 5.3.1. Therefore, the overall efficiency dependence on time is also considered. To reduce the background level, the tagged leg in $\mathrm{Z}(\mathrm{PP})$ is required to have a phoenix track and satisfy all electron ID selection cuts. The efficiency is measured with the other leg, which is defined as a target leg. The efficiency is defined as

$$
\epsilon_{P, P H X(N P H X)}^{P P}(\text { run })=\frac{N_{P P}^{P H X(N P H X)}(\text { run })}{N_{P K}^{P H X(N P H X)}(\text { run })}
$$

where $N_{P P}^{P H X(N P H X)}$ is the number of events with both electrons pass all plug ID 
selection cuts and the target leg does (does not) have a silicon phoenix track associated

with it. The $N_{P K}^{P H X(N P H X)}$ is the number of events for which the target leg passes only the kinematic selection cut and has (does not have) an associated track. The background is subtracted, and it is obtained via the $\mathrm{Z} /$ dielectron mass distribution background method used for the plug ID efficiency in $\mathrm{Z}(\mathrm{CP})$. The plug electron ID efficiency in the $\mathrm{Z}(\mathrm{PP})$ topology as a function of the run period is shown in Figure 5.15 .

\subsubsection{Identification Efficiency in Boson Rapidity}

The electron ID efficiencies of the two electrons are convoluted via the simulation to give the dielectron efficiency vs rapidity $\left(y_{Z}\right)$. The efficiency scale factors (data/MC) applied to the simulation are determined for each electron category separately and the weighting factor for the event is calculated based on the corresponding scale factors. The weighting factors for an electron is $\epsilon^{D a t a} / \epsilon^{M C}$ for electrons passing the ID requirements, and $\left(1-\epsilon^{\text {Data }}\right) /\left(1-\epsilon^{M C}\right)$ for electrons failing the ID requirement. The weighting factor for each MC event is obtained by multiplying the weighting factors for the two electrons. The reweighted MC sample is used to determine the ID efficiency versus Z/dielectron boson rapidity. Figure 5.16 shows the ID efficiency versus boson rapidity for the three event topologies $(\mathrm{Z}(\mathrm{CC}), \mathrm{Z}(\mathrm{CP})$ and $\mathrm{Z}(\mathrm{PP})$ ). 

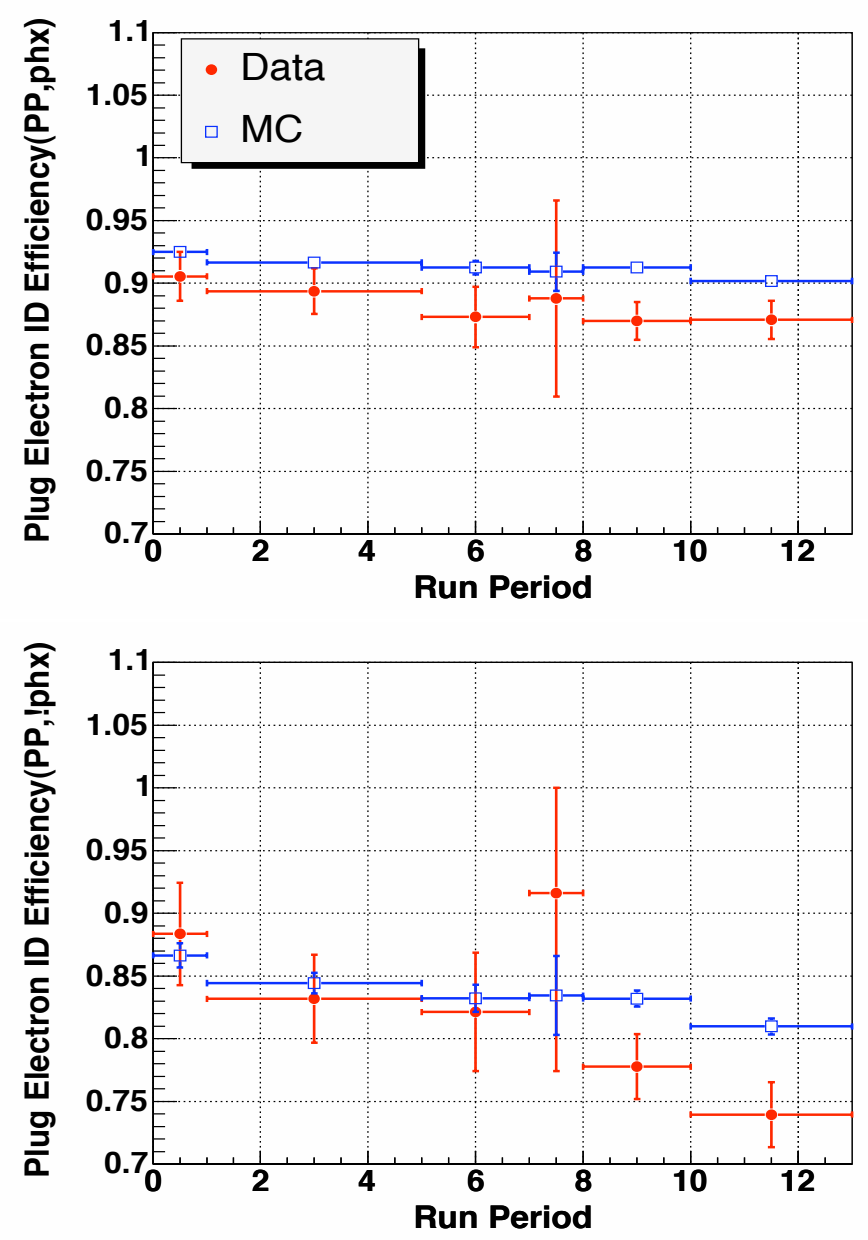

Figure 5.15: The plug electron ID efficiency for $\mathrm{Z}(\mathrm{PP})$ events. The efficiency is measured versus run period. The top plot shows the efficiency for electrons with a phoenix track and the bottom plot shows the efficiency for electrons without a phoenix track. 


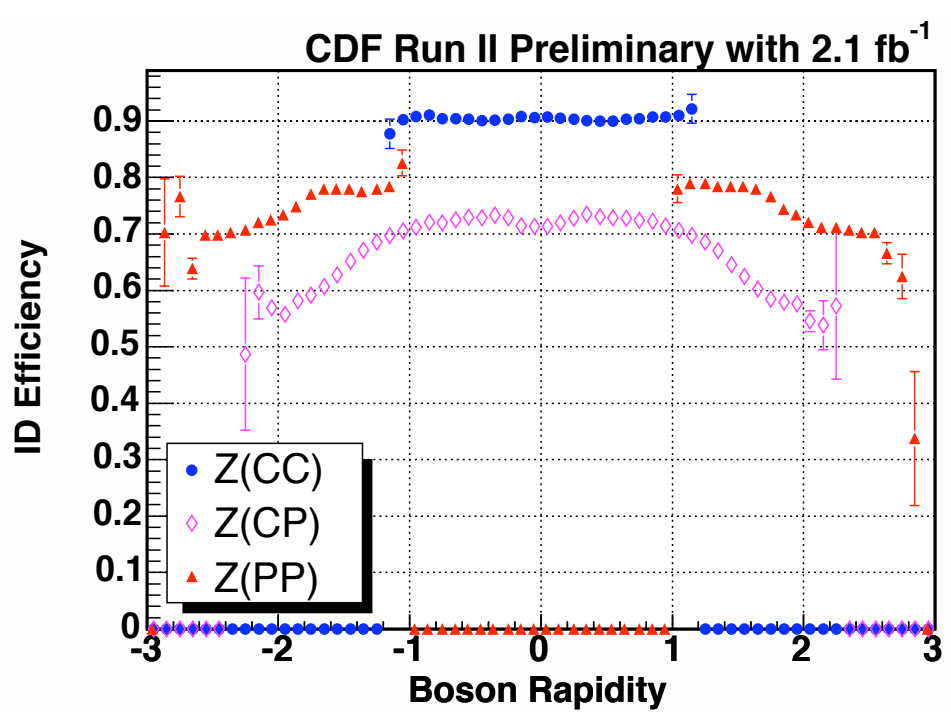

Figure 5.16: The electron ID efficiency distribution versus $\mathrm{Z} /$ dielectron boson rapidity for the three event topologies $(\mathrm{Z}(\mathrm{CC}), \mathrm{Z}(\mathrm{CP})$ and $\mathrm{Z}(\mathrm{PP}))$. The ID efficiency versus $\mathrm{Z}$ boson rapidity is determined by using the tuned $\mathrm{MC}$ simulation. 


\section{Chapter 6}

\section{Systematic Uncertainties}

The following sources contribute to the systematic uncertainty in the measurement of the differential cross section.

- Systematic Error from Modeling of Detector Materials

As particles traverse the detector, they multiple scatter and interact in the detector material producing knock-on electrons and bremsstrahlung photons. These electrons and photons also interact in the material and produce electromagnetic showers. The amount of material in the detector determines the rate of these radiation and showering processes. Monte Carlo simulations for Z/Drell-Yan production, which are generated with additional material in the central and plug regions, are compared to the standard Monte Carlo sample. These simulations are used to determine the systematic uncertainty originating from the modeling of the material in the detector. The Monte Carlo was run with a additional $1 \%$ of a radiation length (X0) of material in the central region, and with an additional $1 / 6 \mathrm{X} 0$ of material in the plug region.

The extra material changes the acceptance as a function of the $\mathrm{Z}$ boson rapidity.

The ratio of the acceptance derived from the Monte Carlo with more material 

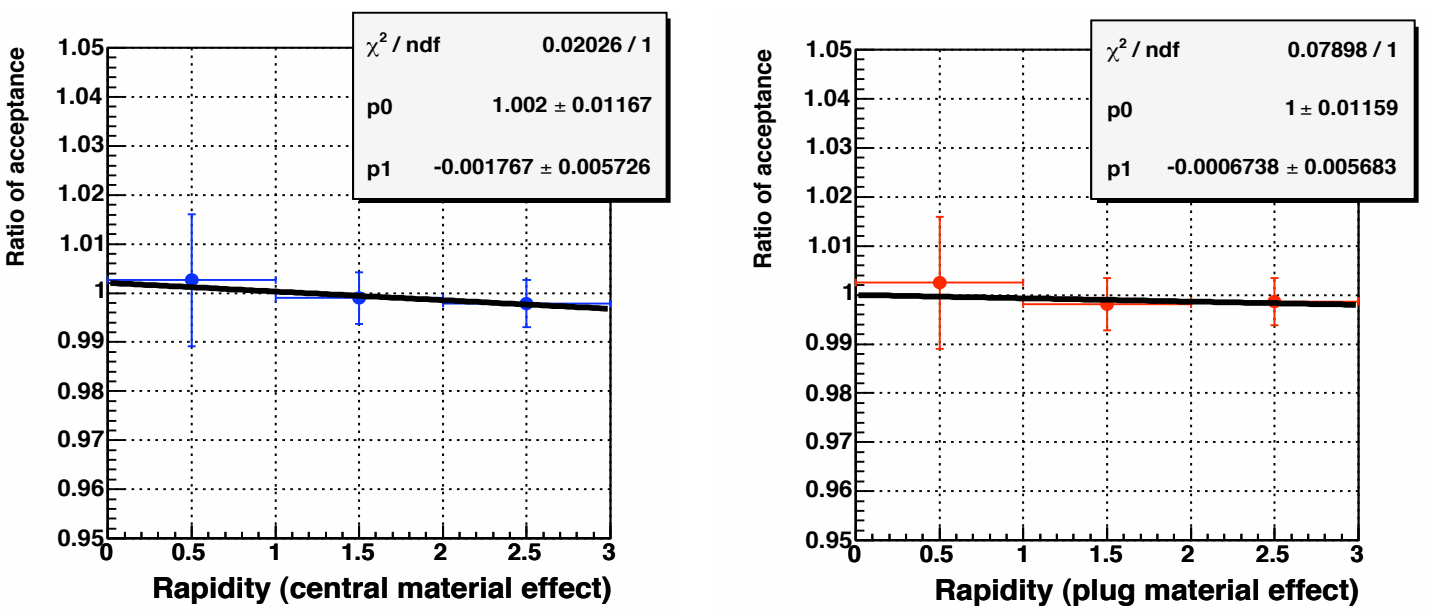

Figure 6.1: The acceptance changes caused by an additional 1\% radiation length $(\mathrm{X} 0)$ of material in the central region (left plot), and with an additional 1/6 X0 of material in the plug region (right plot).

to the acceptance derived from the standard Monte Carlo sample is shown in Figure 6.1. These are used as a measure of the systematic uncertainty in our knowledge of the amount of material in the central and plug regions. Both regions contribute to the overall systematic uncertainty in modeling the detector materials. As the central and plug regions are independent of each other, the systematic uncertainties from modeling the materials in the central and plug region are estimated separately and combined in quadrature.

- Systematic Error on the Estimates of Background

The QCD background is estimated by using both the isolation extrapolation method and from a fit to the dielectron Z/Drell-Yan invariant mass distribution. The rate of the dominant QCD "dijet" background contribution is determined from the isolation extrapolation method. The level of the QCD "dijet" 
background is fixed in the fit to the Z/Drell-Yan mass distribution to extract an estimate of the QCD " $\gamma+j e t$ " background.

For estimating the systematic error, we repeat the fit and allow the level of the QCD "dijet" background in the fit to the dielectron Z/Drell-Yan invariant mass distribution to float, thus simultaneously determining both the level of the QCD QCD "dijet" background and the level of the QCD " $\gamma+$ jet" background from the fit. The difference in the level of the QCD "dijet" fraction in the sample between the level derived from the isolation extrapolation method and the level derived from the Z/Drell-Yan mass fit is used as the first part of the systematic uncertainty in the estimate of the QCD background.

Another contribution to the systematic error originates from uncertainties in the modeling of the dielectron invariant mass distribution of the $\gamma+j e t$ background. The fraction of QCD $\gamma+$ jet background is only determined from the fit to the Z/Drell-Yan invariant mass distribution. The dielectron invariant mass distribution of background events originating from the QCD $\gamma+$ jet process is obtained from a simplified Monte Carlo (MC) sample (without a full simulation of the fragmentation of the final state parton jet and detector simulation). To get a realistic mass spectrum, a fragmentation factor which is constrained by both the $E_{T}$ and isolation energy requirements is applied in selection of events from the QCD $\gamma+$ jet $\mathrm{MC}$ sample. The isolation energy requirement is sensitive to modeling the energy response in the calorimeter to the final state parton jet. Therefore, different assumptions on the the energy response change the allowed phase space for the fragmentation factor. In estimating the background from the QCD $\gamma+$ jet process, we assume that the energy 
response of the calorimeter $(\mathrm{E} / \mathrm{P})$ is 1.0 . We then vary the energy response of the calorimeter by $30 \%$ and determine the background fraction from $\gamma+j e t$ mass spectrum for $E / P=0.7$. The deviation in the background fraction estimated with $E / P=0.7$ and $E / P=1.0$ is used as the second source of systematic uncertainty in the determination of the QCD background. We combine both sources to to yield the uncertainty on the QCD background. Additional details are given in chapter 4 . The change in $d \sigma / d y_{Z}$ from these errors in background fraction is used in the determination of the overall systematic uncertainty.

- Systematic Error in the Efficiency of Electron Identification Cuts

The overall electron identification efficiencies are measured as a function of time for both central and plug electrons. However, the $\eta$ dependence of the electron identification efficiency is only measured for central electrons. The electron ID efficiencies measured in the data are used in the MC by weighting Monte Carlo events with scale factors which are the ratio of efficiencies measured in the data to efficiencies determined in the MC. Here, the main contribution to the systematic uncertainty is from the statistical errors in the measurements of the efficiencies from the data. The statistical uncertainty in the MC sample is negligible. Therefore, only the statistical uncertainties in the data are considered as a source of systematic uncertainty in the electron ID efficiencies. The scale factors corresponding to changing the efficiencies extracted from the data by their standard deviation are applied to the MC sample. The corresponding deviations of $d \sigma / d y_{Z}$ originating from this change in the scale factors are used as the systematic uncertainties in $d \sigma / d y_{z}$ from the uncertainty in the determination of the electron identification efficiencies. The statistical uncertainties for 
each time period are determined separately.

- Systematic Error in the Silicon Tracking Efficiency

We require that at least one of the two plug electrons has a silicon phoenix track. The ratio of the silicon tracking efficiency from the data and MC is applied as a scale factor to Monte Carlo events in order to tune the MC sample to agree with data. The silicon tracker tracking efficiency is determined from the tuned MC sample. There is statistical uncertainty of the efficiency extracted from the data. The deviation of the $d \sigma / d y_{Z}$ measurement from change of one standard deviation in the scale factor is used as the systematic uncertainty in $d \sigma / d y_{Z}$ from the uncertainty in the silicon tracking efficiency.

- Systematic Error in the ZVertex Finding Efficiency

Events in the $\mathrm{Z}(\mathrm{PP})$ topology are required to have a reconstructed ZVertex as one of the kinematic selection cuts. The reconstructed ZVertex finding efficiency is measured for both data and MC samples. The ratio of the ZVertex finding efficiency between data and MC is applied as a scale factor to Monte Carlo events in order to tune the MC sample to agree with data. The tuned (corrected) MC sample is used for the determination of the acceptance and overall efficiency. There is a statistical uncertainty of the ZVertex finding efficiency extracted from the data. The deviation of $d \sigma / d y_{Z}$ resulting from a change of one standard deviation in the measurement of the efficiency using the data is used as the systematic error in $d \sigma / d y_{Z}$ originating from the systematic uncertainty in the ZVertex finding efficiency.

- Plug electron energy calibration 


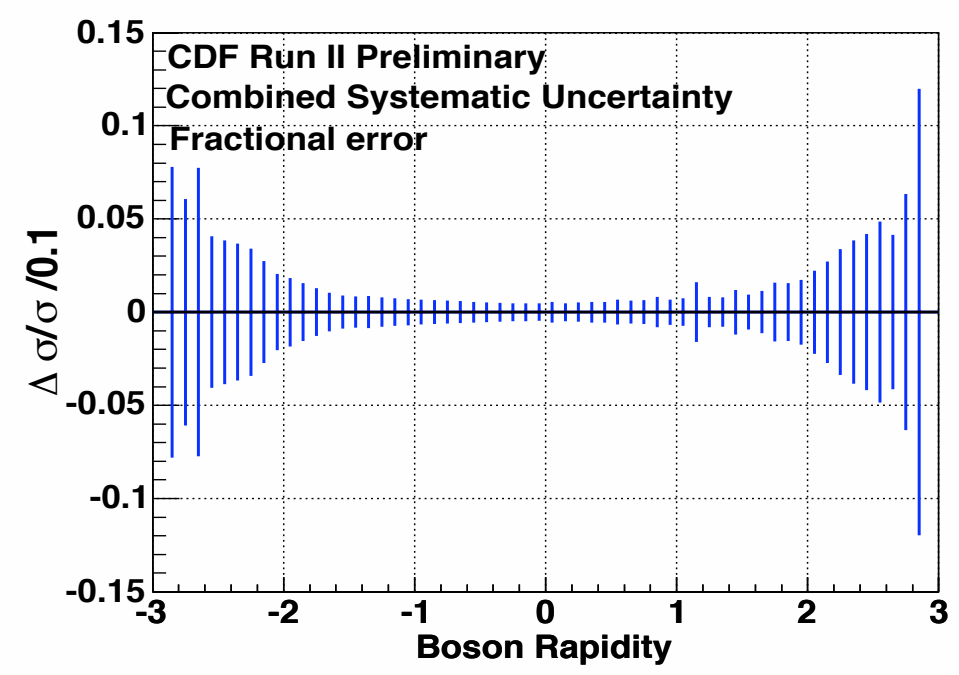

Figure 6.2: The combined systematic uncertainty in $y_{z}$. The systematic uncertainty shown in the plot is the fractional uncertainty.

In the data, the energy as measured in the plug calorimeter for plug electrons for $\eta>0$ is corrected to match the energy of plug electrons for $\eta<0$ The correction on the plug electron energy changes the $\mathrm{Z}$ boson rapidity distribution, and also the number of events selected by the $\mathrm{Z}$ mass selection cuts. The deviation of $d \sigma / d y_{Z}$ resulting from a change of one standard deviation in the energy scale is used as the systematic error in $d \sigma / d y_{Z}$ originating from the uncertainty in the calibration of the plug electron energy scale.

The systematic uncertainties from each component are independent. Therefore, the overall combined systematic error in $d \sigma / d y_{Z}$ is obtained by adding the uncertainties from all sources in quadrature for each $y_{Z}$ bin. Figure 6.2 shows the overall combined systematic uncertainty in $d \sigma / d y_{Z}$ as a function of $y_{Z}$. 


\section{Chapter 7}

\section{Results}

\subsection{The $d \sigma / d y$ Distribution for the $\gamma^{*} / Z \rightarrow$ ee Pro- cess}

The acceptance corrections and corrections for the overall efficiency as a function of the dielectron rapidity are applied to the events passing selection cuts to yield a measurement of the differential cross section for $\gamma^{*} / Z \rightarrow e e$ as a function of the rapidity of the ee pair in the final state..

The differential cross section $d \sigma / d y$ is calculated from the following equation :

$$
\frac{d \sigma\left(\gamma^{*} / Z\right)}{d y}=\frac{\sum_{i=C C, C P, P P} N_{s i g}^{i}(y)-N_{b k g}^{i}(y)}{\sum_{i=C C, C P, P P} A_{Z}^{i}(y) \cdot \epsilon_{t r i g}^{i} \cdot \epsilon_{Z \text { Vertex }} \cdot \epsilon_{Z}^{i}(y) \cdot \int L^{i} d t}
$$

where $A_{Z}$ is the acceptance, $\epsilon_{\text {trig }}$ is the trigger efficiency, $\epsilon_{Z V e r t e x}$ is the efficiency of the $\mid Z$ Vertex $\mid \leq 60 \mathrm{~cm}$ cut, $\epsilon_{Z}$ is the $\mathrm{Z}$ reconstruction efficiency, and $\int L d t$ is the integrated luminosity for the data sample. The Cerenkov Luminosity Counter (CLC)

determines the luminosity over all $z$. However, we apply the cut, $\mid Z V$ ertex $\mid \leq 60 \mathrm{~cm}$ to insure that there is good acceptance of $p \bar{p}$ events for all sub-components of the 
detector. The acceptance of the $\mid Z V$ ertex $\mid \leq 60 \mathrm{~cm}$ cut is measured using two data samples: One collected before, and one after February, 22 2006, respectively. The acceptance of the $\mid Z V$ ertex $\mid \leq 60 \mathrm{~cm}$ before February, 222006 is $95.8 \pm 0.2 \%$, and the acceptance after that date is $96.8 \pm 0.2 \%$. The luminosity acceptance is measured with data samples collected with a special unbiased (minimum bias) trigger. [14] All rapidity dependent acceptance and efficiencies are evaluated as a function of $\left|y_{Z}\right|$ which is defined here as the rapidity of any $e^{+} e^{-}$pair within the $Z$ boson mass range $66<M_{e e}<116 \mathrm{GeV} / c^{2}$.

\subsubsection{Acceptance Correction}

The acceptance $A_{Z, y}$ (as described in section 5.1) is initially calculated using a LO QCD model with CTEQ5L PDFs for each $d \sigma / d y_{z}$ bin. Higher order QCD corrections change the PDFs and also change the expected $d \sigma / d y_{Z}$ distribution, especially in the high $y_{Z}$ region. Therefore, the input theory prediction affects on the acceptance. To remove the model dependence from the acceptance, the acceptance is recalculated using a correction that only relies on data.

At first, the number of events extracted from the data are compared with the predicted number of events from the Monte Carlo tuned for the energy scale and all efficiency corrections. The background subtractions are applied to extract the number of events in the data as a function of dielectron rapidity. Figure 7.1 shows the ratio of the number of events $\left(d N / d y_{Z}\right)$ in data to the Monte Carlo prediction. The ratio is fit to the function:

$$
\begin{array}{lrl}
f(x)=1 & \text { in } x<1 \\
f(x)=1+p 0 \times(x-1)^{2}+p 1 \times(x-1)^{3} & \text { in } x \geq 1
\end{array}
$$




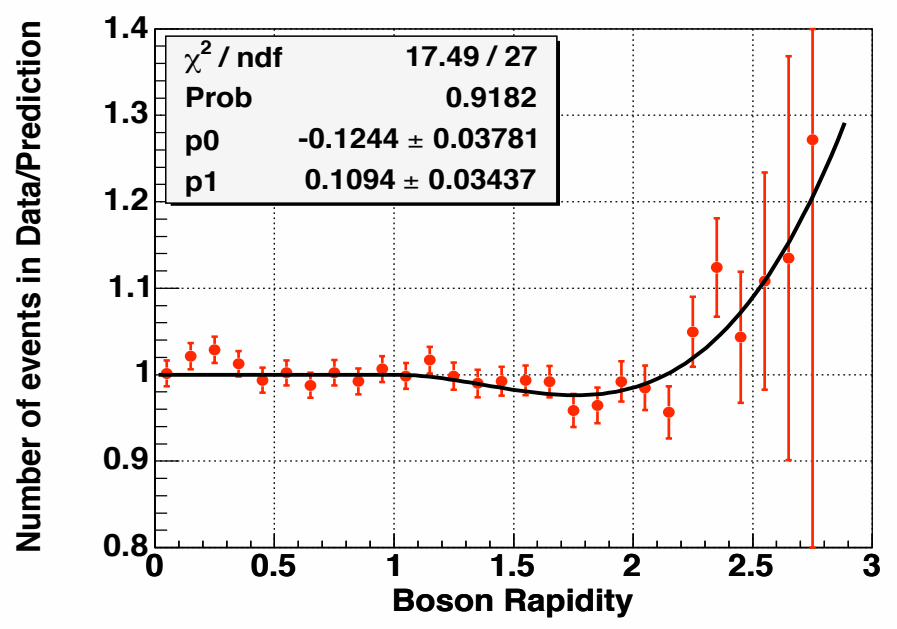

Figure 7.1: The ratio of $d N / d y_{Z}$ in data to Monte Carlo prediction. The fit function is defined as $f(x)=1$ in $x<1$ and $f(x)=1+p 0 \times(x-1)^{2}+p 1 \times(x-1)^{3}$ in $x \geq 1$.

To obtain a corrected acceptance as a function of the $\gamma^{*} / Z \rightarrow$ ee rapidity, a weighting factor from the above fit function is applied to the Monte Carlo events on an event by event. The weighting factor for each event is $f(x)$ where $x$ is the $\gamma^{*} / Z \rightarrow$ ee rapidity at the generator level.

The corrected Monte Carlo is used to get updated values for $d N / d y_{Z}$ in the prediction. These updated values are compared again to the data. The $d N / d y_{Z}$ ratio of data to prediction is re-fitted to get the correction factor in the next level. This procedure is iterated until the ratios to the $d N / d y_{Z}$ data points to predictions are stable and flat. Figure 7.2 shows the ratio of the acceptance corrections before and after the iteration procedure. 


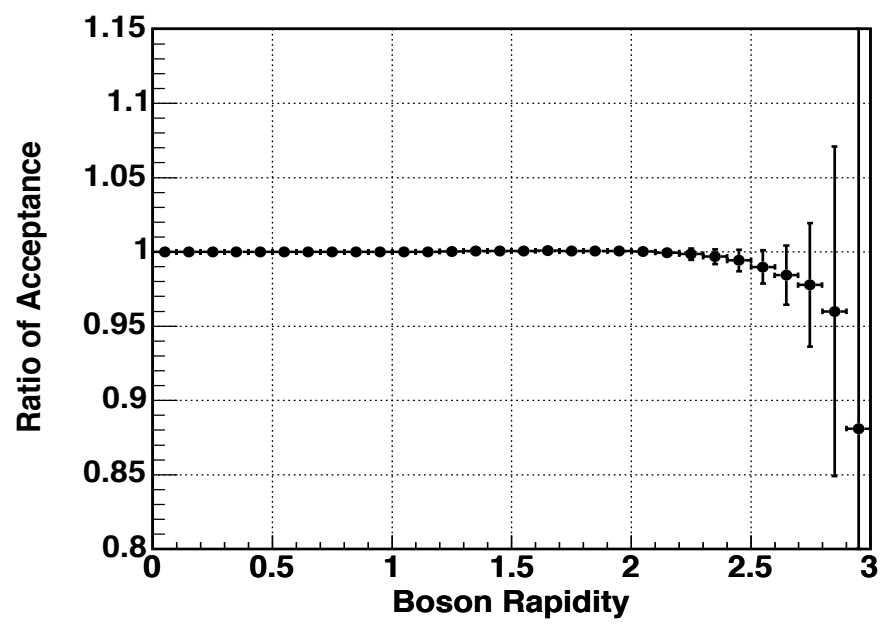

Figure 7.2: The ratio of the final acceptance corrections to correction before the iteration process. Weighting factors using the ratio of $d N / d y_{Z}$ in data to the Monte Carlo prediction are applied to correct the acceptance.

\subsection{2 $d \sigma / d y_{Z}$ Distribution}

After all iterations, the corrected acceptance for each bin (as described in section 7.1.1) is used to to extract a measurement of the differential cross section $d \sigma / d y$ for the production of dileptons from the $\gamma^{*} / Z \rightarrow e e$ process as a function of rapidity. Figure 7.4 shows the measured $d \sigma / d y$ distribution for both $y_{Z} \geq 0$ and $y_{Z}<0$ regions. The $d \sigma / d y$ distributions measured for $y_{Z} \geq 0$ and for $y_{Z}<0$ are consistent with each other within the statistical uncertainty. Therefore, the $d \sigma / d y$ values for both sides are combined together to reduce the statistical uncertainty in $d \sigma / d y$ as a function of the absolute value $\left|y_{Z}\right|$. The combined $d \sigma / d y$ distribution is shown in Figure 7.5. The total cross section for the $\gamma^{*} / Z \rightarrow e e$ process can be measured by integrating the $d \sigma / d y$ distribution. The total cross section for the $\gamma^{*} / Z \rightarrow$ ee process determined by integrating the $d \sigma / d y$ distribution over all $y_{Z}$ in Figure 7.5 is $\sigma=255.69 \pm 0.66 \pm 2.47 p b$. 


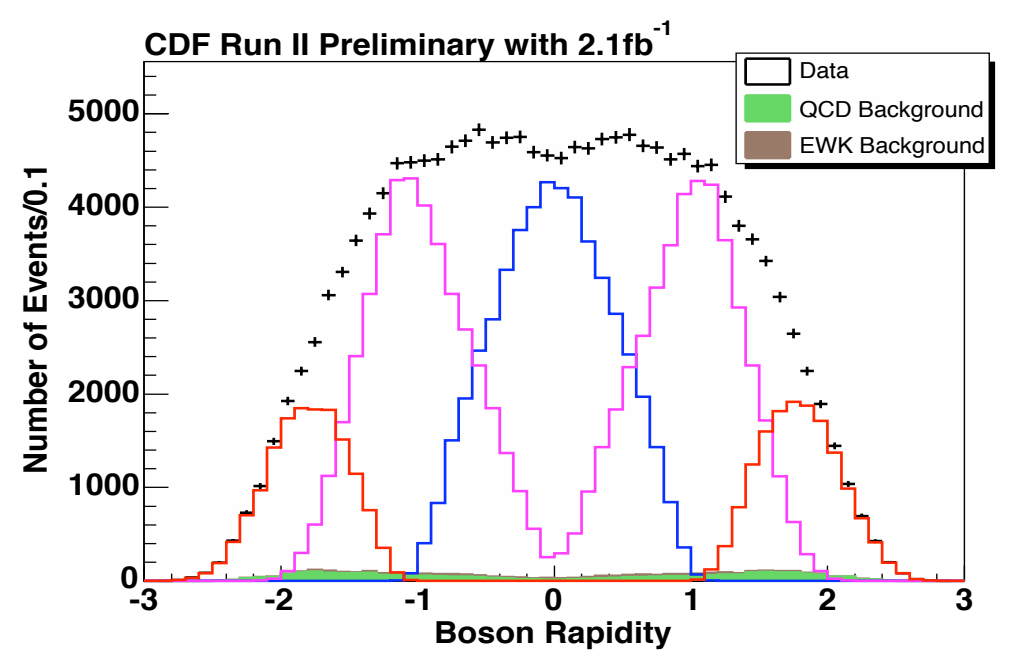

Figure 7.3: The rapidity distribution of $Z^{0} / \gamma^{*} \rightarrow e^{+} e^{-}$dielpton events for different event topologies and for the sum. The background contribution (which is also shown) is very small .

The total cross section is measured to be $\sigma=255.57 \pm 0.96 \pm 2.67 p b$ if only $y_{Z} \geq 0$ events used, and $\sigma=255.84 \pm 0.93 \pm 2.41 p b$ if only $y_{Z}<0$ are used. Table 7.1 shows the measured $d \sigma / d y$ values and experimental uncertainties for each $y_{Z}$ bin.

The $d \sigma / d y$ distributions extracted from events with different topologies $(\mathrm{Z}(\mathrm{CC})$, $\mathrm{Z}(\mathrm{CP})$ and $\mathrm{Z}(\mathrm{PP})$ ) are extracted to check on the consistency between the three topologies. Here, $\mathrm{Z}(\mathrm{CC})$ and $\mathrm{Z}(\mathrm{CP})$ events overlap in the $0<\left|y_{Z}\right|<1$ region, and $\mathrm{Z}(\mathrm{CP})$ and $\mathrm{Z}(\mathrm{PP})$ events overlap in the $1<\left|y_{Z}\right|<2$ region. The comparison of $d \sigma / d y$ distributions in the overlap region provides a systematic check the measurement procedure. The $d \sigma / d y$ distributions separately extracted for each of the three topologies are shown in Figure 7.6. 


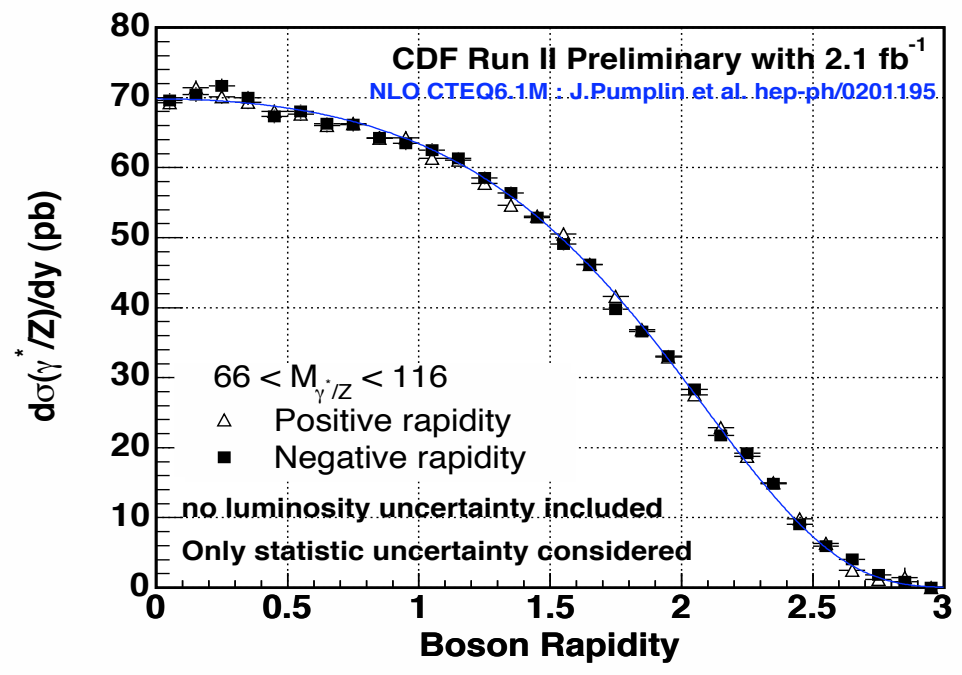

Figure 7.4: The $d \sigma / d y$ distribution for the production of $Z^{0} / \gamma^{*} \rightarrow e^{+} e^{-}$events for $y_{Z} \geq 0$ and for $y_{Z}<0$. The theory prediction is normalized to the total measured cross section in the data. Only statistical uncertainties are shown.

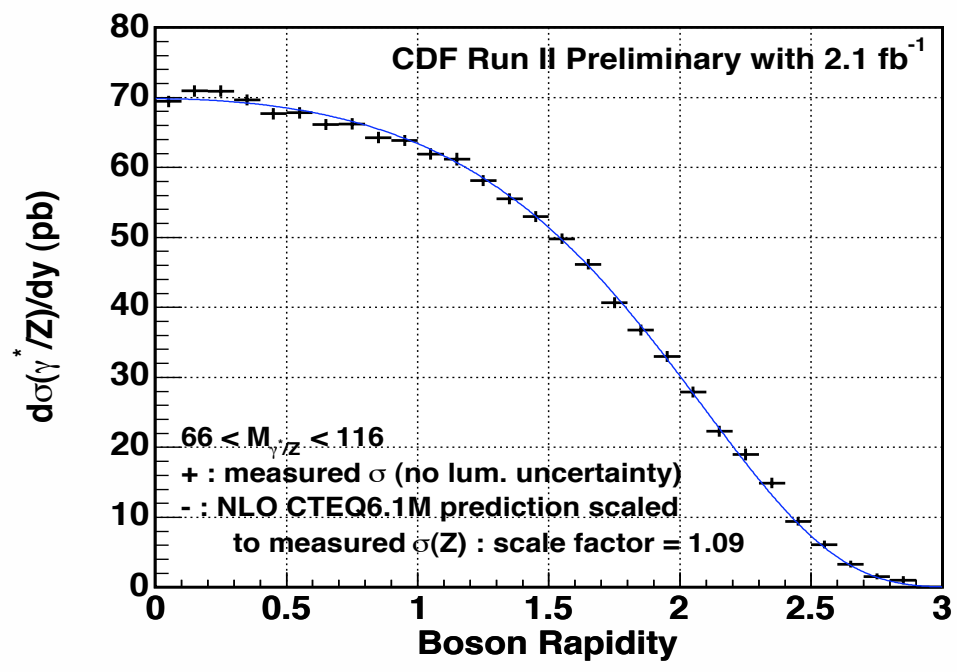

Figure 7.5: The $d \sigma / d y$ distribution for the production of $Z^{0} / \gamma^{*} \rightarrow e^{+} e^{-}$events. The theory prediction is normalized to the total measured cross section in the data. The errors shown in the plot are the combined statistical and systematic uncertainty. 


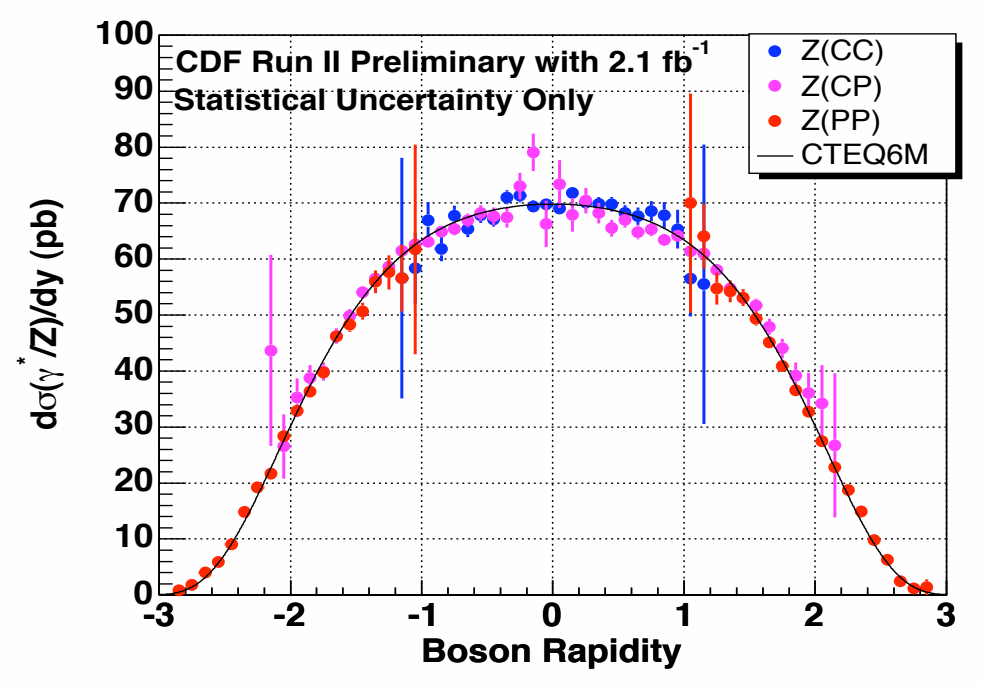

Figure 7.6: The $d \sigma / d y$ distribution for the production of $Z^{0} / \gamma^{*} \rightarrow e^{+} e^{-}$events in $\mathrm{Z}(\mathrm{CC}), \mathrm{Z}(\mathrm{CP})$, and $\mathrm{Z}(\mathrm{PP})$ event topologies. The theory curve is normalized to the measured total cross section in the data.

\subsection{Comparison of Data to Theory}

The ratios of the of the measured $d \sigma / d y$ values to theory are used to compare the results to different theoretical predictions. The main purpose of our measurement is to compare the overall shape of the rapidity distribution between data and theory. Therefore, in all of the plots the theory predictions are normalized to the total measured cross section in the data. Three different theory predictions are used in the comparison. These include NLO and NNLO predictions using the CTEQ6.1M NLO[16], the MRST 2001E NLO[17], and the MRST2006 NNLO [18] PDFs. Figure 7.7 shows the $d \sigma / d y$ ratio of data to theory predictions. Only the statistical uncertainty is shown in the plots and used in the calculation of $\chi^{2}$ comparisons of data to theory. There are bin to bin correlations in the systematic errors. The $\chi^{2}$ for the 
consistency of the $d \sigma / d y$ distribution with the theory predictions is 44 for CTEQ6.1M (NLO), 94 for MRST2001E (NLO), and 59 for the MRST2006 (NNLO) theory prediction (with 30 degrees of freedom). The theory prediction of the NLO calculation with CTEQ6.1M PDFs describes the data better that the other two theoretical models.

Figure 7.8 shows the $d \sigma / d y$ ratio of data to CTEQ6.1M theory prediction for $y \geq 0$ and $y<0$, respectively. The $d \sigma / d y$ ratios for both side agrees with each other to within the statistical uncertainty. 


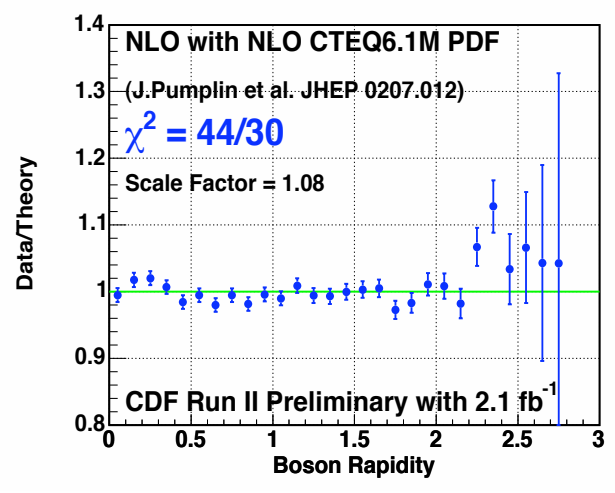

(a) CTEQ6.1M

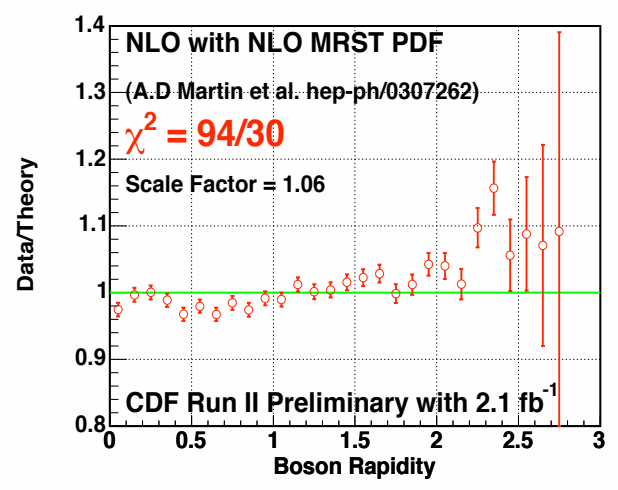

(b) MRST 2001E NLO

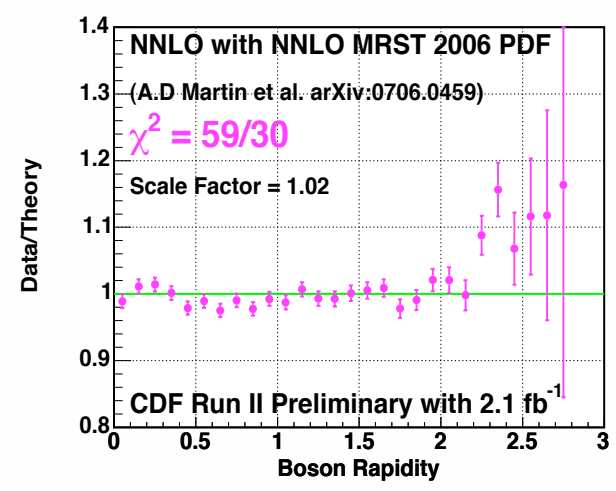

(c) MRST 2006 NNLO

Figure 7.7: The $d \sigma / d y$ ratio of the data to theory predictions. All theory predictions are normalized to the measured total cross section in order to compare the shapes of the distributions between data and theory. Only statistical errors are are shown because there are bin to bin correlations in the systematic errors. Plot (a) is a comparison to CTEQ6.1M NLO, plot (b) is a comparison to MRST 2001E NLO, and plot (c) is a comparison to the MRST2006 NNLO theory prediction. 


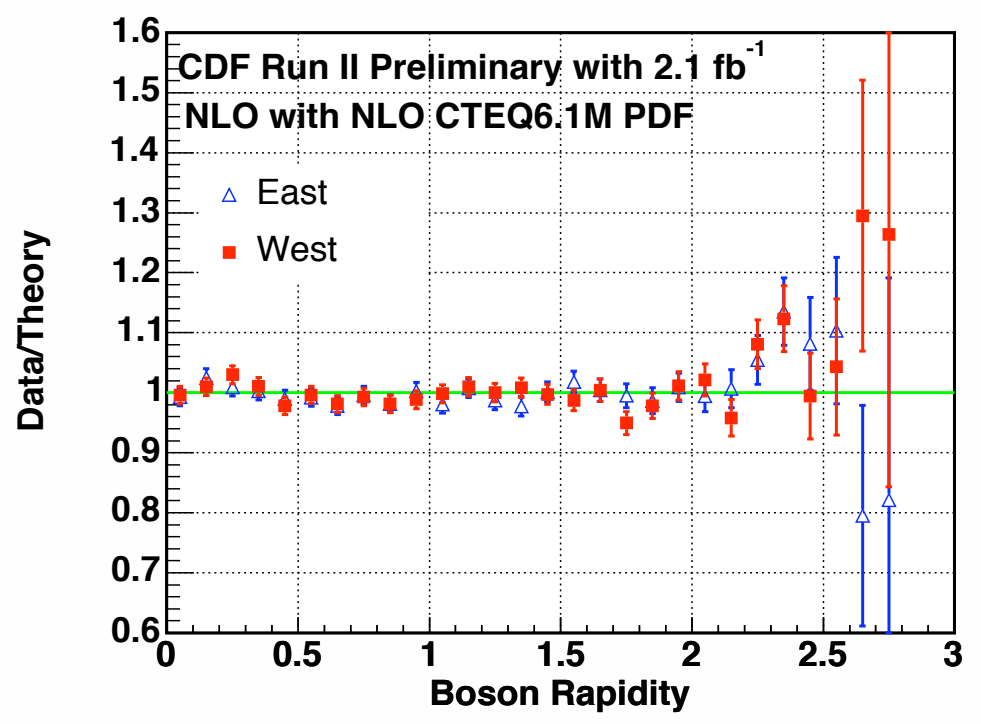

Figure 7.8: The $d \sigma / d y$ ratio of the data to CTEQ6.1M theory predictions for $y \geq$ 0 and $y<0$. The theory predictions are normalized to the measured total cross section in order to compare the shapes of the distributions between data and theory. Only statistical errors are are shown because there are bin to bin correlations in the systematic errors. 


\begin{tabular}{|c||c|c|c||c|c|c|c|c|c|}
\hline $\mathrm{y}$ & $\sigma$ & stat. $\delta$ & sys. $\delta$ & material & BKG & tracking & ID & zvtx & calib \\
\hline \hline 0.05 & 69.44 & 0.73 & 0.34 & 0.13 & 0.05 & 0.00 & 0.29 & 0.00 & 0.11 \\
0.15 & 70.93 & 0.74 & 0.34 & 0.12 & 0.06 & 0.00 & 0.31 & 0.00 & 0.05 \\
0.25 & 70.91 & 0.73 & 0.35 & 0.11 & 0.08 & 0.00 & 0.32 & 0.00 & 0.02 \\
0.35 & 69.66 & 0.72 & 0.36 & 0.10 & 0.11 & 0.00 & 0.33 & 0.00 & 0.04 \\
0.45 & 67.68 & 0.70 & 0.37 & 0.08 & 0.12 & 0.00 & 0.33 & 0.00 & 0.01 \\
0.55 & 67.82 & 0.70 & 0.40 & 0.07 & 0.15 & 0.00 & 0.35 & 0.00 & 0.11 \\
0.65 & 66.12 & 0.69 & 0.39 & 0.06 & 0.16 & 0.00 & 0.35 & 0.00 & 0.05 \\
0.75 & 66.22 & 0.69 & 0.41 & 0.06 & 0.18 & 0.00 & 0.37 & 0.00 & 0.04 \\
0.85 & 64.26 & 0.68 & 0.45 & 0.05 & 0.19 & 0.00 & 0.37 & 0.00 & 0.14 \\
0.95 & 63.87 & 0.68 & 0.43 & 0.05 & 0.19 & 0.00 & 0.38 & 0.00 & 0.02 \\
1.05 & 61.93 & 0.66 & 0.44 & 0.04 & 0.22 & 0.00 & 0.38 & 0.00 & 0.05 \\
1.15 & 61.21 & 0.65 & 0.63 & 0.05 & 0.24 & 0.01 & 0.38 & 0.01 & 0.43 \\
1.25 & 58.14 & 0.65 & 0.46 & 0.05 & 0.29 & 0.00 & 0.36 & 0.03 & 0.04 \\
1.35 & 55.52 & 0.64 & 0.45 & 0.05 & 0.31 & 0.03 & 0.32 & 0.06 & 0.05 \\
1.45 & 52.99 & 0.63 & 0.50 & 0.06 & 0.34 & 0.03 & 0.27 & 0.09 & 0.22 \\
1.55 & 49.82 & 0.62 & 0.44 & 0.06 & 0.33 & 0.10 & 0.22 & 0.12 & 0.10 \\
1.65 & 46.18 & 0.60 & 0.48 & 0.07 & 0.33 & 0.23 & 0.17 & 0.14 & 0.13 \\
1.75 & 40.70 & 0.58 & 0.53 & 0.07 & 0.33 & 0.28 & 0.12 & 0.15 & 0.24 \\
1.85 & 36.76 & 0.56 & 0.55 & 0.07 & 0.30 & 0.41 & 0.08 & 0.16 & 0.12 \\
1.95 & 33.00 & 0.55 & 0.59 & 0.06 & 0.26 & 0.49 & 0.06 & 0.15 & 0.05 \\
2.05 & 27.92 & 0.52 & 0.59 & 0.06 & 0.19 & 0.54 & 0.05 & 0.13 & 0.03 \\
2.15 & 22.29 & 0.50 & 0.61 & 0.05 & 0.19 & 0.56 & 0.04 & 0.11 & 0.03 \\
2.25 & 18.97 & 0.51 & 0.64 & 0.05 & 0.14 & 0.62 & 0.04 & 0.09 & 0.04 \\
2.35 & 14.87 & 0.52 & 0.56 & 0.04 & 0.11 & 0.54 & 0.03 & 0.07 & 0.02 \\
2.45 & 9.40 & 0.48 & 0.38 & 0.03 & 0.06 & 0.37 & 0.02 & 0.05 & 0.00 \\
2.55 & 6.08 & 0.47 & 0.27 & 0.02 & 0.07 & 0.26 & 0.02 & 0.03 & 0.00 \\
2.65 & 3.28 & 0.46 & 0.21 & 0.01 & 0.16 & 0.13 & 0.01 & 0.02 & 0.00 \\
2.75 & 1.50 & 0.41 & 0.09 & 0.01 & 0.07 & 0.06 & 0.01 & 0.01 & 0.00 \\
2.85 & 0.95 & 0.56 & 0.09 & 0.00 & 0.09 & 0.02 & 0.00 & 0.00 & 0.00 \\
2.95 & 0.00 & 0.00 & 0.00 & 0.00 & 0.00 & 0.00 & 0.00 & 0.00 & 0.00 \\
\hline \hline Total & 255.69 & 0.66 & 2.47 & 0.34 & 1.07 & 0.93 & 1.20 & 0.28 & 0.43 \\
\hline
\end{tabular}

Table 7.1: Table of values for the $d \sigma / d y$ measurement with the overall statistical and systematic errors. The systematic uncertainties from different sources are also in the table. 


\section{Chapter 8}

\section{Conclusion}

We report on a measurement of the rapidity distribution, $d \sigma / d y$, for Z/Drell-Yan $\rightarrow e e$ events produced in $p \bar{p}$ collisions at $\sqrt{s}=1.96 \mathrm{TeV}$. The data sample consists of 2.13 $\mathrm{fb}^{-1}$, corresponding to about about 160,000 Z/Drell-Yan $\rightarrow e e$ candidates collected by the Collider Detector at Fermilab during the first part of Run II. The $d \sigma / d y$ distribution is measured over the full kinematic range for $e^{+} e^{-}$pairs in the $Z$ boson mass range $66<M_{e e}<116 \mathrm{GeV} / c^{2}$.

Three different event topologies are used in the measurement. The number of events corresponds to 50784, 86230, and 31346 events, for the $\mathrm{Z}(\mathrm{CC}), \mathrm{Z}(\mathrm{CP})$, and $\mathrm{Z}(\mathrm{PP})$ topologies, respectively. The $\mathrm{Z}(\mathrm{CC})$ is the events reconstructed with two central electrons, the $\mathrm{Z}(\mathrm{CP})$ is the events reconstructed with a central and a plug electron, and the $\mathrm{Z}(\mathrm{PP})$ is the events reconstructed with two plug electrons. The $\mathrm{Z}(\mathrm{PP})$ topology corresponds to the high rapidity region and makes it possible to probe the high momentum fraction region of the parton distribution functions of quarks in the nucleon. The integrated luminosity of the data is $2128.1 p b^{-1}$ for the $\mathrm{Z}(\mathrm{CC})$ and $\mathrm{Z}(\mathrm{CP})$ topologies, and $2020.3 p b^{-1}$ for $\mathrm{Z}(\mathrm{PP})$ (because of the silicon tracking

requirement for $\mathrm{Z}(\mathrm{PP})$ ). The acceptance and efficiencies are measured in the data as 
a function of rapidity of the dielectron pair $\left(y_{Z}\right)$, and the corrections are applied as a function of $y_{Z}$. A summary of the number of signal and background events (after all selection cuts) as a function of $y_{Z}$ is given in Table 8.1.

The measurement is compared with QCD predictions in Next to Leading Order (NLO), and Next to Next to Leading Order (NNLO), calculated with the CTEQ6.1M (NLO), MRST2001E (NLO), and MRST2006 (NNLO) parton distribution functions, respectively. There is good agreement with the NLO QCD theory predictions with CTEQ6.1M PDFs. The data indicate a somewhat higher cross section than the theory predictions in the highest rapidity region $\left(y_{Z}>2.2\right)$. However, the statistical sample at high rapidity is not sufficient to investigate $d \sigma / d y$ in detail in that region.

This measurement of $d \sigma / d y$ of $Z \rightarrow e e$ can be used in QCD global fits that extract parton distributions functions in the nucleon from various sets of data. 


\begin{tabular}{|c||c|c|c||c|c|c||c|c|c|}
\hline $\mathrm{y}$ & $\mathrm{N}(\mathrm{CC})$ & $\mathrm{N}(\mathrm{CP})$ & $\mathrm{N}(\mathrm{PP})$ & $\mathrm{B}(\mathrm{CC})$ & $\mathrm{B}(\mathrm{CP})$ & $\mathrm{B}(\mathrm{PP})$ & $\mathrm{S}(\mathrm{CC})$ & $\mathrm{S}(\mathrm{CP})$ & $\mathrm{S}(\mathrm{PP})$ \\
\hline \hline 0.05 & 8514 & 566 & 0 & 46 & 16 & 0 & 8468 & 550 & 0 \\
0.15 & 8142 & 1092 & 0 & 37 & 27 & 0 & 8105 & 1065 & 0 \\
0.25 & 7423 & 1960 & 0 & 31 & 45 & 0 & 7392 & 1915 & 0 \\
0.35 & 6605 & 2866 & 0 & 31 & 65 & 0 & 6574 & 2801 & 0 \\
0.45 & 5684 & 3758 & 0 & 26 & 74 & 0 & 5658 & 3684 & 0 \\
0.55 & 4909 & 4697 & 0 & 23 & 99 & 0 & 4886 & 4598 & 0 \\
0.65 & 3942 & 5428 & 0 & 18 & 117 & 0 & 3924 & 5311 & 0 \\
0.75 & 2947 & 6338 & 0 & 13 & 124 & 0 & 2934 & 6214 & 0 \\
0.85 & 1685 & 7341 & 0 & 9 & 139 & 0 & 1676 & 7202 & 0 \\
0.95 & 766 & 8301 & 0 & 3 & 144 & 0 & 763 & 8157 & 0 \\
1.05 & 155 & 8742 & 24 & 2 & 152 & 0 & 153 & 8590 & 24 \\
1.15 & 12 & 8691 & 221 & 0 & 163 & 7 & 12 & 8528 & 214 \\
1.25 & 0 & 7510 & 751 & 0 & 149 & 23 & 0 & 7361 & 728 \\
1.35 & 0 & 6125 & 1607 & 0 & 126 & 61 & 0 & 5999 & 1546 \\
1.45 & 0 & 4817 & 2484 & 0 & 104 & 92 & 0 & 4713 & 2392 \\
1.55 & 0 & 3505 & 3224 & 0 & 90 & 113 & 0 & 3415 & 3111 \\
1.65 & 0 & 2313 & 3785 & 0 & 73 & 137 & 0 & 2240 & 3648 \\
1.75 & 0 & 1280 & 3925 & 0 & 48 & 173 & 0 & 1232 & 3752 \\
1.85 & 0 & 613 & 3880 & 0 & 29 & 156 & 0 & 584 & 3724 \\
1.95 & 0 & 223 & 3594 & 0 & 13 & 140 & 0 & 210 & 3454 \\
2.05 & 0 & 51 & 2890 & 0 & 4 & 89 & 0 & 47 & 2801 \\
2.15 & 0 & 13 & 2041 & 0 & 2 & 85 & 0 & 11 & 1956 \\
2.25 & 0 & 0 & 1427 & 0 & 0 & 54 & 0 & 0 & 1373 \\
2.35 & 0 & 0 & 856 & 0 & 0 & 26 & 0 & 0 & 830 \\
2.45 & 0 & 0 & 397 & 0 & 0 & 10 & 0 & 0 & 387 \\
2.55 & 0 & 0 & 169 & 0 & 0 & 5 & 0 & 0 & 164 \\
2.65 & 0 & 0 & 54 & 0 & 0 & 4 & 0 & 0 & 50 \\
2.75 & 0 & 0 & 14 & 0 & 0 & 1 & 0 & 0 & 13 \\
2.85 & 0 & 0 & 3 & 0 & 0 & 0 & 0 & 0 & 3 \\
2.95 & 0 & 0 & 0 & 0 & 0 & 0 & 0 & 0 & 0 \\
\hline \hline Total & 50784 & 86230 & 31346 & 238 & 1804 & 1177 & 50546 & 84426 & 30169 \\
\hline
\end{tabular}

Table 8.1: The number of signal and background events in each topology. $\mathrm{N}$ is the total number of events, B is the number of the background events, $\mathrm{S}$ is the number of signal events. $(\mathrm{S}=\mathrm{N}-\mathrm{B})$ 


\section{Appendix A}

\section{Glossary}

CDF Collider Detector at Fermilab.

CEM Central Electromagnetic Calorimeter.

CES Central Electromagnetic Showermax.

COT Central Outer Tracker.

CPR Central Preshower Radiator.

MET Missing Transverse Energy.

Parton Quarks and gluons that comprise protons and neutrons.

PDF Parton Distribution Function.

PEM Plug Electromagnetic Calorimeter.

PES Plug Electromagnetic Showermax.

PPR Plug Preshower Radiator.

QCD Quantum Chromodynamics. 
QED Quantum Electrodynamics. 


\section{Bibliography}

[1] A combination of preliminary electroweak measurements and constraints on the standard model, (2003).

[2] S.D. Drell and T.-M. Yan, Phys. Rev. Letter. 25 (1970) 316.

[3] G. Altarelli, R.K. Ellis and G. Martinelli, Nucl. Phys. B157 (1979) 461.

[4] J. Kubar, M. le Bellac, J.L. Meunier and G. Plaut, Nucl. Phys. B175 (1980) 251.

[5] P. Aurenche and P. Chiappetta, Z. Phys. C34 (1987) 201.

[6] P.J. Sutton, A.D. Martin, R.G. Roberts, W.J. Stirling, Phys. Rev. D45 (1992) 2349.

[7] E.W.N. Glover, hep-ph/0211412, and references therein.

[8] P.J. Rijken and W.L. van Neerven, Phys. Rev. D. D51 (1995) 44

[9] Charalampos Anastasiou, Lance Dixon, Kirill Melnikov, and Frank Petriello, "Dilepton rapidity distribution in the Drell-Yan process at NNLO in QCD", Phys. Rev. Letter. 91 (2003) 182002, hep-ph/0306192

[10] Charalampos Anastasiou, Lance Dixon, Kirill Melnikov, and Frank Petriello, "High-precision QCD at hadron colliders: electroweak gauge boson rapidity distributions at NNLO”, Phys. Rev. D. 69 (2004) 094008, hep-ph/0312266 
[11] Kevin Pitts, The CDF Collaboration, "The CDF Central Outer Tracker", FERMILAB-CONF-96, 443-E (1996).

[12] G. Apollinari, The CDF Collaboration, "CDF Plug Upgrade Shower Maximum Detector Local Geometry", CDF/DOC/PLUG_UPGR/CDFR/5774, (2001).

[13] Veronique Boisvert, The CDF Collaboration, "Trigger Efficiencies for the High $E_{T}$ Central Electrons in Gen6", CDF/DOC/ELECTRON/CDFR/7939 (2006).

[14] Willis Sakumoto "The Event $Z_{v t x}$ Cut $\left(\left|Z_{v t x}\right|<60 \mathrm{~cm}\right)$ Acceptance for RUN II", CDF/ANAL/ELECTROWEAK/CDFR/8318 (2006).

[15] Torbjorn Sjostrand et al., "High energy physics event generation with PYTHIA 6.1", Comput. Phys. Commun. 135 (2001), 238-259.

[16] J. Pumplin, D.R. Stump, J. Huston, H.L. Lal, P. Nadolsky, and W.K. Tung, "New Generation of Parton Distributions with Uncertainties from Global QCD Analysis", JHEP 0207 (2002), 012, hep-ph/0201195.

[17] A.D. Martin, R.G. Roberts, W.J. Stirling, and R.S. Thorne, "Uncertainties of Predictions from Parton Distributions. I: experimental errors", Eur. Phys. J. C28 (2003), hep-ph/0307262.

[18] A.D. Martin, W.J. Stirling, R.S. Thorne, and G. Watt, "Update of Parton Distribution at NNLO", arXiv: 0706.0459 (2006).

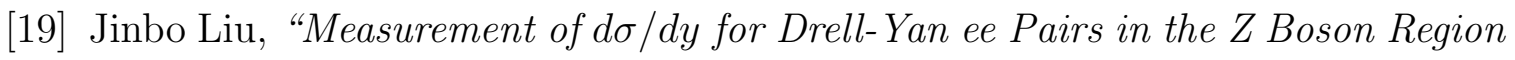
at CDF", CDF/THESIS/ELECTOWEAK/PUBLIC/5405 (2000). 